9.直直目目

FBS

36

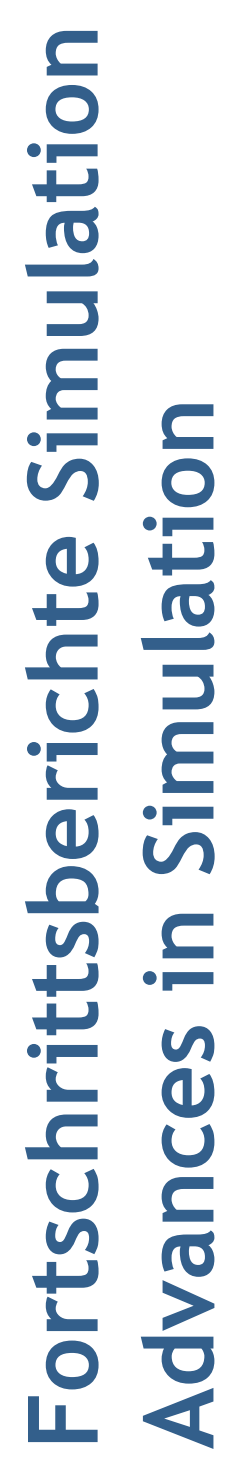

\title{
Konforme Abbildungen zur Simulation von Modellen mit verteilten Parametern
}

\section{Martin Holzinger}

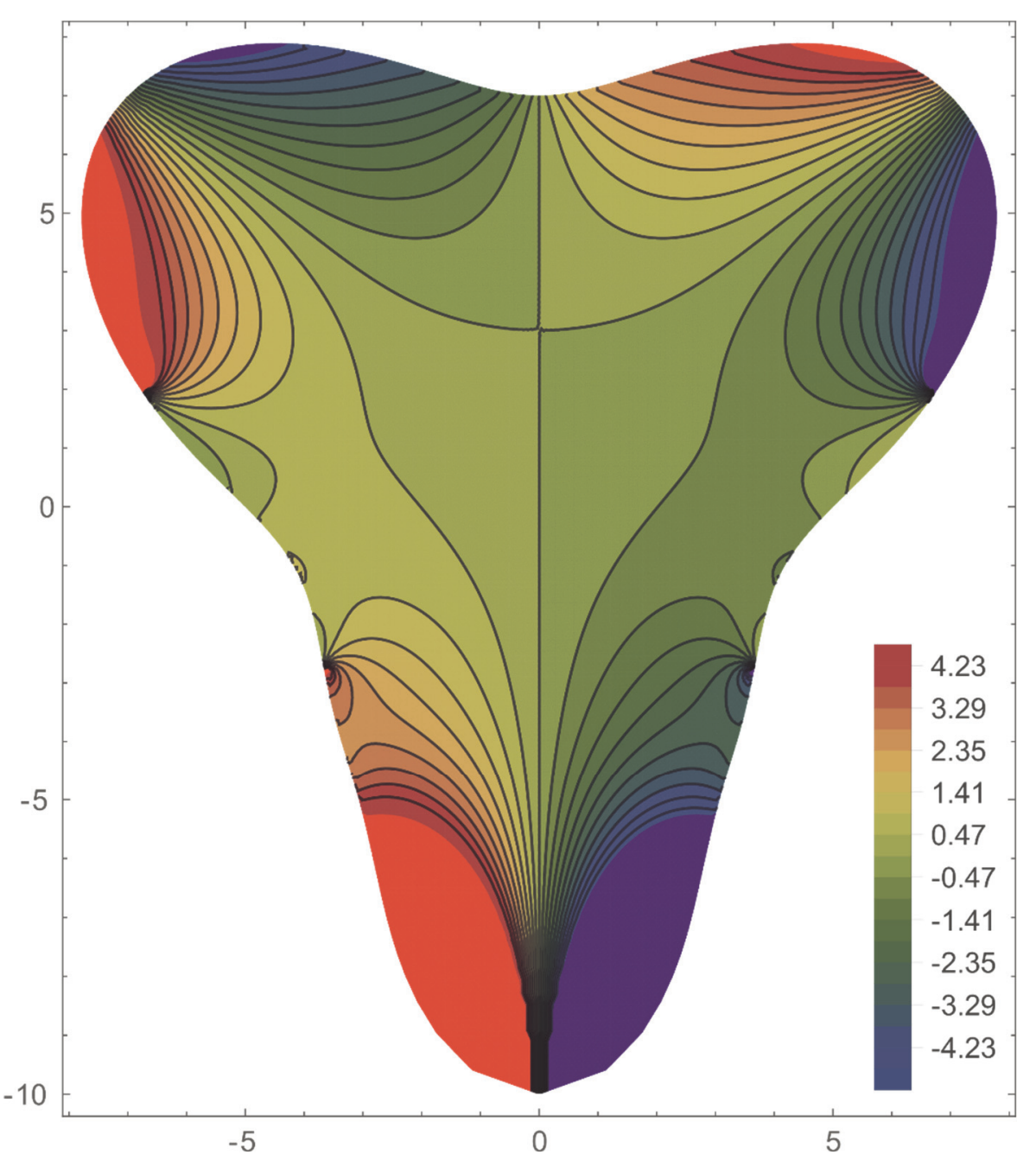

ISBN print 978-3-903311-10-7 ISBN ebook 978-3-903347-36-6 DOI 10.11128/fbs.36 



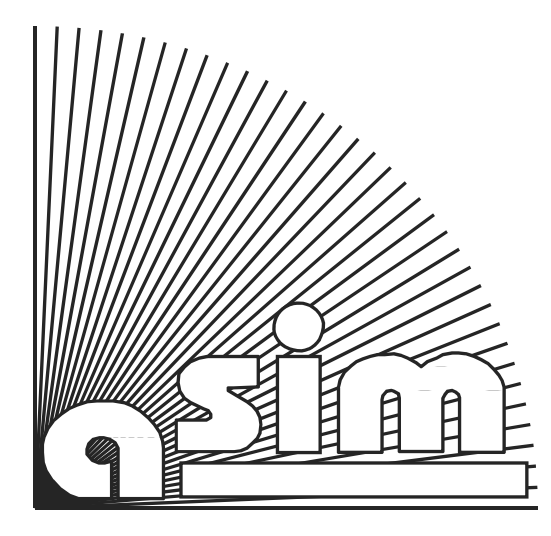

ARGESIM 



\title{
FBS Fortschrittsberichte Simulation Advances in Simulation
}

Herausgegeben von ASIM - Arbeitsgemeinschaft Simulation, Fachausschuss der GI - Gesellschaft für Informatik - im Fachbereich ILW - Informatik in den Lebenswissenschaften Published by ASIM - German Simulation Society, Section of GI - German Society for Informatics iin Division ILW - Informatics in Life Sciences

\section{ASIM FBS 36}

\author{
Martin Holzinger
}

\section{Konforme Abbildungen zur Simulation von Modellen mit verteilten Parametern}




\section{FBS - Fortschrittsberichte Simulation / Advances in Simulation}

Herausgegeben von ASIM - Arbeitsgemeinschaft Simulation, $\rightarrow$ www.asim-gi.org ASIM ist ein Fachausschuss der GI - Gesellschaft für Informatik, $\rightarrow$ www.gi.de, im Fachbereich ILW Informatik in den Lebenswissenschaften, $\rightarrow$ fb-ilw.gi.de

Published on behalf of ASIM - German Simulation Society, $\rightarrow$ www.asim-gi.org ASIM is a section of of $\mathbf{G I}-$ German Society for Informatics, $\rightarrow$ www.gi.de, in division ILW - Informatics in the Life Sciences, $\rightarrow$ fb-ilw.gi.de

\section{Reihenherausgeber / Series Editors}

Prof. Dr.-Ing. Th. Pawletta (ASIM), HS Wismar, Thorsten.pawletta@hs-wismar.de

Prof. Dr. D. Murray-Smith (EUROSIM / ASIM), Univ. Glasgow,

David.Murray-Smith@glasgow.ac.uk

Prof. Dr. F. Breitenecker (ARGESIM / ASIM), TU Wien, Felix.Breitenecker@tuwien.ac.at

Titel / Title: Konforme Abbildungen zur Simulation von Modellen mit verteilten Parametern

Autor /Author: Martin Holzinger, martin.holzinger@tuwien.ac.at

FBS Band / Volume: 36

Typ / Type: Dissertation

Supervisor / Reviewer: Felix Breitenecker, TU Wien, Felix.Breitenecker@tuwien.ac.at Dirk Praetorius, TU Wien, Dirk.Praetorius@tuwien.ac.at

Thorsten Pawletta, HS Wismar,Thorsten.Pawletta@hs-wismar.de

ISBN print: 978-3-903311-10-7, TU-Verlag, Vienna, 2020;

Print-on-Demand, www.tuverlag.at

ISBN ebook: 978-3-903347-36-6, ARGESIM Publisher Vienna, 2020;

www.argesim.org

DOI: $10.11128 / \mathrm{fbs} .36$

Seiten / Pages: vi +79 pages 


\title{
TECHNSCHE UNIVERSITÄT \\ W I E N WIEN
}

\section{DISSERTATION}

\section{Konforme Abbildungen zur Simulation von Modellen mit verteilten Parametern}

\author{
Ausgeführt zum Zwecke der Erlangung des akademischen Grades eines \\ Doktors der technischen Wissenschaften \\ unter Leitung von
}

Ao. Univ.-Prof. Dipl.-Ing. Dr. Felix Breitenecker

Institut für Analysis und Scientific Computing

eingereicht an der Technischen Universität Wien

Fakultät für Mathematik und Geoinformation

von

Dipl.-Ing. Martin Holzinger

08826417

A-4844 Regau 2 



\section{Dissertation Martin Holzinger - Kurzfassung}

Die vorliegende Arbeit soll aufzeigen, in welcher Weise die klassische konforme Abbildung zur Simulation ebener bzw. symmetrischer Anfangsrandwertprobleme nutzbringend eingesetzt werden kann. Die zugrunde liegende Methodik bedient sich dabei der Parametrisierung eines zweidimensionalen Gebietes durch das Einheitsquadrat, wobei der mit numerischen Methoden ermittelten konformen Abbildung die analytisch bekannte Transformation des Quadrates auf den Einheitskreis vorgeschaltet wird. Durch die derart bewerkstelligte Einführung geeigneter Koordinaten wird die Verwendung regelmäßiger Rechengitter ermöglicht. Auf diesen Gitterstrukturen leistet die Linienmethode zur Diskretisierung des Ortes eine Überführung der partiellen in ein System von gewöhnlichen Differentialgleichungen. Letztere werden mit Standardverfahren zur Integration im Zeitbereich gelöst. Für numerische Berechnungen wird durchgehend das Programm Mathematica 11.3 eingesetzt.

Dem Mehraufwand der einmaligen numerischen Berechnung und Abspeicherung der durch die Einführung „konformer Koordinaten“ bedingten, zusätzlich benötigten metrischen Größen in den Rechengitterpunkten stehen gravierende Vorteile gegenüber, die die Herangehensweise an derartige Problemstellungen rechtfertigen: Ausgehend von einer koordinatenfreien Formulierung verhält sich etwa der Laplace-Operator bis auf einen ortsabhängigen Faktor invariant unter der konformen Abbildung. Einfach gestaltet sich auch die Implementierung von Randbedingungen, da der nach außen gerichtete Einheitsnormalenvektor stets mit der Richtung einer Koordinatenlinie zusammenfällt.

Zur Demonstration der prinzipiellen Vorgehensweise wird im ersten Kapitel eine analytische Lösung eines Wärmeleitungsproblems am Einheitsquadrat mittels Orthogonalreihenansatz entwickelt, das für alles weitere benötigte Rechengitter aufgesetzt und die darauf mit der CTDS-Methode gewonnene Simulationslösung mit der Reihenlösung und jener der FE-Methode von Matlabs PDEToolbox verglichen. Es folgt die Angabe eines Konstruktionsverfahrens zur Diskretisierung von Ortsableitungen beliebiger Ordnung unter Berücksichtigung der Konvergenzordnung.

Das zweite und dritte Kapitel widmen sich der konformen Abbildung in Theorie und Praxis. Nach Darlegung grundlegender Konzepte der Funktionentheorie werden die benötigten konstruktiven Verfahren vorgestellt: Abbildungen vom Schwarz-Christoffel-Typ für Polygonzüge stellen eine analytische Transformation des Quadrates auf den Kreis sicher, aus der Fülle der konstruktiven Verfahren wird sodann die Theodorsensche Integralgleichung aufgegriffen und mit den Mitteln einer modernen Programmierumgebung erfolgt die hochgenaue Berechnung der Ränderzuordnungsfunktion vom Einheitskreis auf sternförmige Gebiete nebst Fehlerbetrachtungen, und zwar für die gespiegelte Ellipse, das Einheitsquadrat selbst sowie drei allgemeinere Geometrien mit $\varepsilon$-Bedingung.

Im vierten Kapitel werden in den ersten beiden Abschnitten die Grundlagen zur Anwendung der konformen Koordinatentransformation vorbereitet, die nächsten beiden Abschnitte widmen sich der speziellen Gestalt der Metrik und den Randbedingungen. Da durch die Hintereinanderschaltung der numerisch ermittelten konformen Abbildung mit der Jacobischen Amplitudenfunktion die metrischen Größen der Transformation sämtlich bekannt sind, kann die Wärmeleitungsgleichung in den neuen Koordinaten simuliert werden. Für ein Problem am Einheitskreis wird dazu wieder eine Reihenlösung entwickelt, Zugänge zum Simulationsmodell werden für Kreis und Quadrat in den letzten beiden Abschnitten demonstriert und mit den exakten Lösungen verglichen.

Das fünfte Kapitel verwertet die Ergebnisse zu einer Simulationsstudie: Ermittelt wird die Temperaturverteilung in einem mittels Laserquelle behandelten Zahn. Das Quadratgitter wird dazu konform auf den Zahnquerschnitt abgebildet, anschließend kann durch Einführung von Zylinderkoordinaten eine dreidimensionale Modellerweiterung realisiert werden. Die Wärmeabgabe in das umliegende Knochengewebe bzw. an die Luft wird durch geeignete Randbedingungen modelliert. Die Applikation der Wärmequelle mittels Laserimpuls findet in der Vorgabe eines Quellterms mit Maximum auf der Rotationsachse ihre Entsprechung. Abschließende theoretische Betrachtungen gelten der Verallgemeinerung der Wärmeleitungsgleichung, durch den Diffusionstensor können Anisotropien und Inhomogenitäten der Wärmeausbreitung Berücksichtigung finden.

Der Epilog erarbeitet Abgrenzung zur und Argumente für die FE-Methode, berichtet über weitere Untersuchungen und betrachtet noch einmal die Theodorsengleichung hinsichtlich Genauigkeit. 


\section{Doctoral Thesis Martin Holzinger - Short Abstract}

Purpose of the thesis is to demonstrate how conformal mappings can be utilized in simulation of twodimensional or symmetric three-dimensional Initial Boundary Value Problems. Parametrization of a 2D-region by means of the unit square is achieved when combining the analytically given map from unit square to unit disk with numerically constructed conformal transformations. The introduction of such special co-ordinates thus makes the usage of rectangular structured computational grids possible. On the other hand on such computational structures the method of lines can be used to transform a system of partial differential equations to a system of ordinary differential equations. The latter can be treated by standard methods available for systems with lumped parameters. All numerical computations are carried out by using the program Mathematica 11.3.

Whilst the computational effort in calculating and saving the specific metric quantities caused by the transformation on each gridpoint has only to be done once for a given geometry, benefits especially arise with respect to, e.g., transformation of the Laplacian operator or the implementation of boundary conditions. That is, an outward pointing normal derivative always coincides with a tangent direction of a co-ordinate line.

In the first chapter, for demonstration purposes an analytical series solution of a heat transfer problem on the unit square is presented and compared with the CTDS simulation solution on a rectangular computational grid as well as with the results obtained by Matlabs PDE-Toolbox. Conclusively, the spacial discretization method is essentially extended: regarding functions in two independent variables, a general construction guideline for substituting spatial derivatives of arbitrary order (including error estimates) is given. Furthermore, it is shown that boundary conditions can easily be implemented by using spacial approximation formulae for non-central grid points.

Chapter two and three focus on the conformal transformation in theory and practice. A brief survey concerning the basics of complex analysis is followed by the presentation of SchwarzChristoffel-type mappings which are being used to analytically establish the transformation from square to disk. Theodorsen's integral equation is then considered to numerically construct a highly accurate solution for the function of boundary correspondence. The unit disk is subsequently being mapped with Mathematica to selected star-shaped regions such as square, inverted ellipse and three more general geometries satisfying an $\varepsilon$-condition.

In chapter four the basics for applying the conformal transformation of co-ordinates are being developed in the first two sections. Starting with a co-ordinate free formulation the differential operators for the particular case of a given conformal parametrization are examined, that is, composition of the numerically obtained conformal map with the Jacobi amplitude yields all further required metric quantities. In section four the proper implementation of boundary conditions is being investigated. Next, a heat conduction problem given on the unit disk is again solved analytically and compared with both the CTDS method and Matlabs PDE-Toolbox. Also, it is shown that with respect to the simulation results on the unit square, no further errors are introduced as long as calculation of the conformal metric is accurate enough.

In chapter five the obtained results are applied to simulate the temperature distribution in the interior of a tooth treated with a laser source. The computational grid is mapped onto a region representing the dental cross section whose boundary can be described by means of interpolation of pre-given boundary points. Introduction of cylindrical co-ordinates yields a realistic model in three dimensions where heat transfer from the interior dental region to surrounding media such as bone and air is modeled by appropriate boundary conditions whereas the heat source itself can be modeled by adding a dynamic source term to the equation. Taking the existence of microscopic dental channels into consideration, an extension of the heat equation finally introduces anisotropic and inhomogeneous heat conduction by means of the diffusion tensor.

The epilogue comments on more recent developments concerning the FE-method, reports further investigations on other geometries and returns to Theodorsen's equation concluding with remarks on possibilities to increase accuracy with respect to calculation of boundary correspondence. 


\section{Inhaltsverzeichnis}

$\begin{array}{lll}\text { Vorwort } & \text { v }\end{array}$

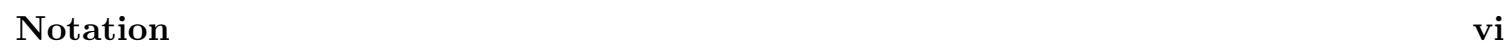

\begin{tabular}{lll}
\hline & Wärmeleitung am Einheitsquadrat & 1
\end{tabular}

1.1 Problemformulierung . . . . . . . . . . . . . . . . . . . 2

1.2 Analytische Lösung . . . . . . . . . . . . . . . . . . . . 3

1.3 Lösung des Problems mit der CTDS-Linienmethode $\ldots \ldots \ldots \ldots$. . . . . . 7

1.4 Vergleich der Simulationslösungen mit dem Reihenansatz . . . . . . . . . . . . . . . 11

$1.5 \quad$ Finite Differenzen . . . . . . . . . . . . . . . . . . . . . . . . . . . . . . . . 14

$1.6 \quad$ Fazit und Argumente für die konforme Abbildung. . . . . . . . . . . . . . . . . 18

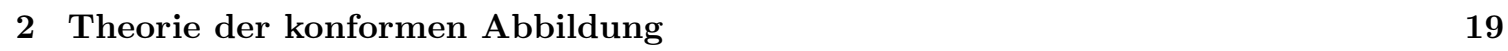

2.1 Elemente der Funktionentheorie . . . . . . . . . . . . . . . . . . . 20

2.2 Randwerte von CAUCHY-Integralen . . . . . . . . . . . . . . . . . 21

2.3 Die konforme Abbildung . . . . . . . . . . . . . . . . . . . . . . . . . . 22

2.4 Transformationen vom SCHWARZ-CHRISTOFFEL Typ . . . . . . . . . . . . . . . 23

2.5 Konforme Abbildung Einheitsquadrat auf Einheitskreis . . . . . . . . . . . . . . . 24

2.6 Die Integralgleichung von THEODORSEN $\ldots \ldots \ldots \ldots \ldots \ldots \ldots \ldots$

\begin{tabular}{|lrr}
3 & Praxis der konformen Abbildung & 29
\end{tabular}

3.1 Numerische Behandlung der Gleichung von THEODORSEN . . . . . . . . . . . . . . 30

$3.1 .1 \quad$ Die Methode der sukzessiven Approximation $\ldots \ldots \ldots$. . . . . . . . 30

$3.1 .2 \quad$ Das verallgemeinerte Newtonverfahren . . . . . . . . . . . . . . . . . . 31

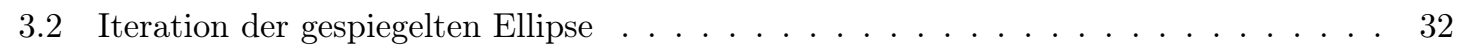

$3.3 \quad$ Numerische Lösung der Quadratabbildung . . . . . . . . . . . . . . . . . . 35

3.4 Abbildung auf ein rotationssymmetrisches Gebiet . . . . . . . . . . . . . . . . 37

3.4 .1 Definition der Geometrien . . . . . . . . . . . . . . . . . . . . . . 37

3.4 .2 Die Newtonmethode von HÜBNER nach WEGMANN . . . . . . . . . . . . . . . 39

3.4 .3 Bemerkungen zur Implementierung . . . . . . . . . . . . . . . . . . . 40

3.4 .4 Ergebnisse $\ldots \ldots \ldots \ldots \ldots \ldots$ 
4 Anwendung konformer Koordinatentransformationen 45

4.1 Der Riemannsche Raum . . . . . . . . . . . . . . . . . . . . . . . . . . . 46

4.2 Differentialoperatoren im Riemannschen Raum . . . . . . . . . . . . . . . . . . . . . 49

4.3 Metrische Größen der konformen Abbildung . . . . . . . . . . . . . . . . . 50

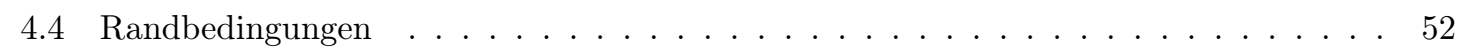

4.5 Wärmeleitung am Einheitskreis . . . . . . . . . . . . . . . . . . . . . 54

4.6 Quadratwärmeleitung revisited (experimentum crucis) $\ldots \ldots \ldots \ldots$. . . . . . . 56

5 Studie: Wärmeleitung im Zahn $\quad 59$

5.1 Anatomische Grundlagen $\ldots \ldots \ldots \ldots$

5.2 Entwicklung des Grundmodells $\ldots \ldots \ldots \ldots \ldots$. . . . . . . . . . . 60

5.3 Parameterüberlegungen und Modellerweiterungen . . . . . . . . . . . . . . 65

$5.3 .1 \quad$ Erhebung realistischer Parameter . . . . . . . . . . . . . . . 65

5.3 .2 Modellierung der Wärmequelle $\ldots \ldots \ldots 6$

5.3 .3 Ergebnisse . . . . . . . . . . . . . . . . . . 66

5.3 .4 Inhomogenitäten und Anisotropien . . . . . . . . . . . . . . . 67

$\begin{array}{lll}6 & \text { Epilog } & \mathbf{7 1}\end{array}$

6.1 Vergleich mit alternativen Finite-Elemente-Verfahren . . . . . . . . . . . . . . . 71

6.2 Einsatz auf verschiedenen weiteren Geometrien . . . . . . . . . . . . . . . . 73

6.3 Genauigkeitssteigerung der Ränderzuordnungsfunktion $\ldots \ldots \ldots \ldots$. . . . . . 76

$\begin{array}{ll}\text { Literaturverzeichnis } & 77\end{array}$

\begin{tabular}{ll}
\hline Curriculum Vitae & 79
\end{tabular} 


\section{Vorwort}

Seit dem Erscheinen meiner Diplomarbeit [19] im Jahre 1996 ist einige Zeit verstrichen und es ist faszinierend, die seither erfolgten Entwicklungen aus dem Blickwinkel jener Zeit zu betrachten. Diese Fortschritte betreffen weniger die mathematische Theorie als vielmehr die Versionsvielfalt höherer Programmiersprachen, die mittlerweile zu einem Grad gereift sind, daß der rückblickende Vergleich auf den damaligen Code und die nunmehrige Leichtigkeit der Implementierungsmöglichkeiten sowie die nahezu beliebige Steuerbarkeit der Genauigkeit in Erstaunen versetzen. Mit Hilfe des Sprachpakets PVM/FORTRAN wurde damals die Beheizung eines Raumes und der umliegenden Wände unter Verwendung der Linienmethode simuliert sowie auf Parallelisierbarkeit untersucht, im Laufe der Jahre entwickelte sich der Ausgangscode sukzessive über C und C++ hin zu Mathematica. Unter dem Gesichtspunkt dieses steten Wandels der zum Einsatz kommenden Werkzeuge und aus Gründen der Kompaktheit wurde auf eine gesonderte Anfügung des Quellcodes in Form eines Anhanges verzichtet, essentielle Snippets finden daher zumeist nur in Form von Fußnoten ihren Eingang.

Fast ein Vierteljahrhundert später möchte ich mit der vorliegenden Arbeit den ursprünglichen Gedanken, abseits der etablierten Methoden die seinerzeit eingesetzte Linienmethode auch auf unregelmäßigen Geometrien in der Simulation partieller Differentialgleichungen zur Anwendung zu bringen, zum Abschluß bringen. Die initialen Anregungen dazu (und noch sehr viel anderes mehr) verdanke ich FELIX BREITENECKER, dem Betreuer dieser Dissertation. Er hat sich auch schon für die Aufgabe der Betreuung meiner Diplomarbeit zur Verfügung gestellt und war mir wissenschaftlich wie menschlich stets ein großes Vorbild. Für Geduld, Ermutigung, Korrektur und langjährige Begleitung der Arbeit ein herzliches Dankeschön! Weiters gilt mein Dank HANs Jörg DirschmiD, der stets bereit war, seinen Wissensreichtum in Form wertvoller Anregungen einzubringen.

Erst durch die erwähnten Fortschritte der höheren Programmiersprachen selbst ist die Zeit gereift, konforme Abbildung und Linienmethode verschmelzen und - ein essentieller Aspekt - auch mit der zu Gebote stehenden Genauigkeit implementieren zu können. Zudem war es mir aus privaten wie beruflichen Gründen nicht immer möglich, die Arbeit mit der nötigen Kontinuität voranzutreiben. Diese Kontinuität wurde sichergestellt durch die Inanspruchnahme einer einjährigen Bildungskarenz. Es soll daher nicht unerwähnt bleiben, daß eine solche Möglichkeit zur geförderten Weiterbildung in Österreich angeboten wird, jedoch in anderen Staaten überwiegend kein Äquivalent findet.

Das genaue Korrekturlesen eines wissenschaftlichen Textes verlangt der eigenen Erfahrung nach ein hohes Maß an Akribie und ist zeitintensiv. Katharina Hutter, IRMgard Husinsky und BERNHARD Simon haben sich zu dieser mühevollen Arbeit bereiterklärt. MANFRED SALZMANN hat sich ebenfalls dieser Aufgabe angenommen, meinem langjährigen Freund und Weggefährten verdanke ich darüber hinaus zahlreiche fruchtbringende Diskussionen inhaltlicher Natur sowie Vorschläge zu Struktur und Aufbau. Also, we together weathered many a storm.

Fräulein Pauline Steinkogler, einem meiner Mädchen, danke ich für die Durchsicht der Arbeit und das Betrachten und Kommentieren der bunten Bilder. Sie verfügt mittlerweile über fundierte Kenntnisse im Zahlenraum $10^{5}$ und es würde den Vater mit großem Stolz erfüllen zu wissen, daß eines Tages auch die Darlegung des verbleibenden kleinen Restes Verständnis findet.

Sehr schwer vorstellbar ist die Fertigstellung der Arbeit ohne die Motivation und mannigfaltige Unterstützung von SusAnNe HiLBERT. Sie ist und bleibt das zweite meiner Mädchen. Ich sag's Dir. 


\section{Notation}

$\mathfrak{Q}, \overline{\mathfrak{Q}} \quad$ offenes/abgeschlossenes Einheitsquadrat als Punktmenge

$\mathfrak{Q}_{n, n} \quad$ diskretes Rechengitter am Einheitsquadrat mit $n^{2}$ Punkten; äquidistant

$\mathfrak{E}, \overline{\mathfrak{E}} \quad$ offener/abgeschlossener Einheitskreis als Punktmenge

$\mathfrak{G}$ Gebiet als offene zusammenhängende Menge (komplexer Zahlen)

$\mathfrak{C} \quad$ Randkurve eines Gebietes als Menge (komplexer Zahlen)

$(\xi, \eta) \quad$ kartesische Koordinaten eines Punktes $P$

$(x, y) \quad$ allgemeine Koordinaten eines Punktes $P$

$\kappa_{\zeta}, \kappa_{z} \quad$ Karten zur Parametrisierung einer Punktmenge (kartesisch, allgemein)

$\zeta=\xi+i \eta \quad(\xi, \eta)$ als komplexe Zahl in $\overline{\mathfrak{G}}$

$w=u+i v \quad(u, v)$ als komplexe Zahl in $\overline{\mathfrak{E}}$

$z=x+i y \quad(x, y)$ als komplexe Zahl in $\overline{\mathfrak{Q}}$

$r e^{i \varphi}, \rho e^{i \theta} \quad$ Polardarstellungen von Punkten aus $\mathfrak{E}$ bzw. aus $\mathfrak{G}$

$r(\varphi), \rho(\theta) \quad$ Parametrisierung des Randes von $\mathfrak{E}$ bzw. von $\mathfrak{C}$

$\theta(\varphi) \quad$ Ränderzuordnungsfunktion; Winkel $\varphi$ am Einheitskreis

$\sigma(\theta) \quad \frac{\rho^{\prime}(\theta)}{\rho(\theta)}$

$\mathfrak{D} \quad$ Diffusionstensor mit Koordinaten $d^{i j}$

$\chi \quad$ abkürzend für $\xi_{x}^{2}+\eta_{x}^{2}=\left|f^{\prime}(z)\right|^{2}$ bei konformer Abbildung $\zeta=f(z)$ 


\title{
Kapitel 1
}

\section{Wärmeleitung am Einheitsquadrat}

\author{
PDEs are good for the soul... [trad.]
}

Bei der Vorgehensweise zur Entwicklung eines Computerprogrammes in Mathematica, dessen Aufgabe die Simulation von Anfangsrandwertaufgaben auf strukturierten Rechengittern sein soll, ist es angebracht, sich zu Vergleichszwecken einer Referenzlösung zu bedienen, damit die notwendigen Implementierungsschritte einerseits auf ihre Korrektheit geprüft, andererseits die Rechenergebnisse der Simulation sowohl quantitativ, als auch hinsichtlich ihrer Qualität bewertet werden können.

Eine solche Referenzlösung wird zunächst für den Fall der instationären Wärmeleitung am Quadrat mit adiabatischer Isolierung auf drei Seiten und einem der Normalenableitung proportionalen Wärmeabfluß auf der Unterseite in Form eines sehr schnell konvergierenden Reihenansatzes entwickelt. Es stellt sich heraus, daß die Lösung unabhängig von einer Koordinatenrichtung ist.

Die Linienmethode liegt der Implementierung des Simulationsmodells zu Grunde und wird im Anschluß präsentiert. Mit ihrer Hilfe wird die instationäre partielle Differentialgleichung durch einen Ortsdiskretisierungsprozeß, bei dem der LaplaCEsche Operator durch Finite Differenzen in jedem Punkt im Inneren des Rechengitters ersetzt wird durch einen nur noch zeitabhängigen Temperaturterm, überführt in ein System gewöhnlicher Differentialgleichungen, das von Mathematica aufintegriert werden kann. Auch die Randbedingungen werden einer solchen Diskretisierung unterworfen, sodaß ein simulationsfähiges Modell entsteht.

Nach Vorstellung dieser Methode, die sich eines regelmäßig strukturierten Rechengitters bedient, um in dessen Gitterpunkten durch Ortsdiskretisierung zu einer Näherungslösung zu gelangen, werden die Ergebnisse der Simulation präsentiert. Ein Vergleich mit der Reihenlösung gibt dabei Aufschluß über die erzielbare Konvergenzordnung bei Verwendung unterschiedlicher Diskretisierungsmethoden unter Einsatz verschieden feiner Rechengitter.

Ein gänzlich anderer Ansatz zur Behandlung partieller Differentialgleichungen wird in der PDEToolbox von Matlab gewählt. Mittels der Methode der Finiten Elemente ist das vorliegende Problem ebenfalls einer Simulationslösung zugänglich, es drängt sich daher ein Vergleich der unterschiedlichen Herangehensweisen auf. Dabei zeigt sich neben der höheren erzielbaren Fehlerordnung der Methode der Finiten Differenzen auch, daß sich die vorgestellte Methode durch eine höhere Flexibilität hinsichtlich der Klasse der behandelbaren partiellen Differentialgleichungen auszeichnet.

Die grundlegende Vorgehensweise der Ersetzung der Ortsableitungen durch ihre diskreten Äquivalente macht es wünschenswert, formelmäßige Ausdrücke für Ableitungen beliebiger Ordnung unter Kenntnis der Fehlerordnung an der Hand zu haben. Der abschließende Abschnitt widmet sich daher der Entwicklung solcher Formeln, wobei im vorliegenden zweidimensionalen Fall auch gemischte partielle Ableitungen Berücksichtigung finden. 


\subsection{Problemformulierung}

Wir suchen die von der Zeit abhängige skalare Temperaturverteilung $u(x, y, t)$ auf dem offenen Einheitsquadrat $\mathfrak{Q}:=(-1,1) \times(-1,1)$ für beliebige Zeitpunkte $t \in(0, \infty)$, auf dessen Innerem demnach die instationäre Wärmeleitungsgleichung

$$
\frac{\partial u}{\partial t}=\kappa \Delta u
$$

erfüllt sein soll. Wir fordern die Lösung für die Anfangsverteilung

$$
u(x, y, 0)=1, \quad \forall x, y \in \mathfrak{Q},
$$

während auf der Berandung von $\mathfrak{Q}$

$$
\begin{array}{ll}
\nabla_{\mathbf{n}} u(1, y, t)=0, & y \in(-1,1), t \in(0, \infty) \\
\nabla_{\mathbf{n}} u(-1, y, t)=0, & y \in(-1,1), t \in(0, \infty) \\
\nabla_{\mathbf{n}} u(x, 1, t)=0, & x \in(-1,1), t \in(0, \infty) \\
\nabla_{\mathbf{n}} u(x,-1, t)+\gamma u(x,-1, t)=0, & x \in(-1,1), t \in(0, \infty), \gamma \in \mathbb{R}
\end{array}
$$

zu gelten habe und beziehen uns im folgenden auf kartesische Koordinaten. Es handelt sich hierbei um die Vorgabe einer Anfangstemperaturverteilung zum Zeitpunkt $t=0$, sowie um homogene Randbedingungen vom Neumannschen beziehungsweise NEwTonschen Typ, bei denen Bedingungen an die Ortsableitungen in Richtung des Einheitsnormalenvektors vorgeschrieben werden. Das vorliegende Anfangsrandwertproblem ist erst durch sämtliche Forderungen (1.1)-1.6 korrekt gestellt. Die Lösung wird allgemein entwickelt, zum Vergleich mit den Computersimulationen mögen dann die Setzungen $\gamma=\kappa=1$ und $t=4$ als Standardfall betrachtet werden.

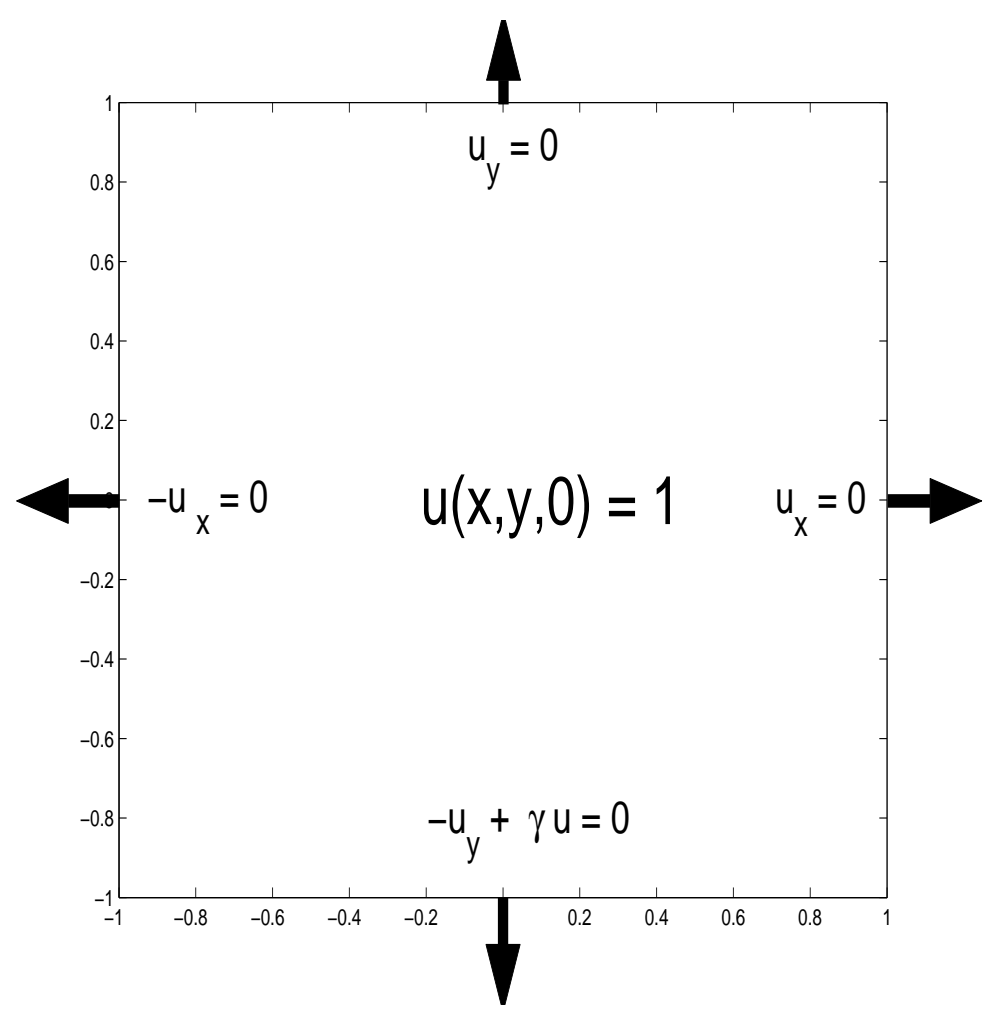

Abbildung 1.1: Illustration der Problemstellung. 


\subsection{Analytische Lösung}

Zur Lösung des Problems berufen wir uns darauf, daß sich jede auf $\mathfrak{Q}$ zweimal stetig differenzierbare Funktion - insbesondere also auch die gesuchte Lösung $u(x, y, t)$ selbst — in eine Orthogonalreihe $u(x, y, t)=\sum_{l=0}^{\infty} U_{l}(x, y) \int u(\xi, \eta, t) U_{l}(\xi, \eta) d \xi d \eta$ nach den orthonormierten Eigenfunktionen des (negativen) Laplace-Operators entwickeln läßt, $-\triangle U_{l}=\lambda_{l} U_{l}$.

Da $\mathfrak{Q}$ beschränkt und der negative Laplace-Operator regulär auf $\mathfrak{Q}$ ist, resultiert ein diskretes Spektrum. Die Zeitvariable $t$ ist dabei als Parameter aufzufassen. Die Anwendung des Separationsansatzes für das Eigenwertproblem mit Hilfe der Produktdarstellung $U(x, y)=X(x) Y(y)$ gibt nach Einsetzen und Division $X^{\prime \prime} / X+Y^{\prime \prime} / Y=-\lambda$, sodaß man auf zwei STURM-LiouvilLEsche Eigenwertaufgaben, $-X^{\prime \prime}=\mu X$ und $-Y^{\prime \prime}=\nu Y, \quad \lambda=\mu+\nu$, geführt wird. Die Existenz der Konstanten $\mu$ und $\nu$ kann aus dem Umstand geschlossen werden, daß beide Quotienten jeweils nur Funktionen einer Veränderlichen (in $x$ bzw. $y$ ) definieren, deren Summe aber konstant zu sein hat. Für Differentialgleichungen dieses Typs existieren, wie man durch Einsetzen leicht verifiziert, Integralbasen der Form $\{\exp (i z \sqrt{\sigma}), \exp (-i z \sqrt{\sigma})\}$, durch Linearkombination folgt

$$
X(x)=A e^{i x \sqrt{\mu}}+B e^{-i x \sqrt{\mu}}, \quad Y(y)=C e^{i y \sqrt{\nu}}+D e^{-i y \sqrt{\nu}} .
$$

Zur Darstellung der speziellen Lösung sind die Randbedingungen heranzuziehen, wobei die Fälle $\mu=0$ und $\nu=0$ die Eindeutigkeit der Lösungsverhältnisse bestimmen und gesondert zu untersuchen sind. Stellt man diese Forderungen an die Funktion $U(x, y)$, so erhält man

$$
\begin{aligned}
& U_{x}(1, y)=X^{\prime}(1) Y(y)=0, \quad-U_{x}(-1, y)=-X^{\prime}(-1) Y(y)=0, \\
& U_{y}(x, 1)=X(x) Y^{\prime}(1)=0, \quad-U_{y}(x,-1)+\gamma U(x,-1)=-X(x) Y^{\prime}(-1)+\gamma X(x) Y(-1)=0,
\end{aligned}
$$

und daraus für die eindimensionalen Teilprobleme

$$
X^{\prime}(1)=-X^{\prime}(-1)=Y^{\prime}(1)=0 \quad \text { sowie } \quad \gamma Y(-1)-Y^{\prime}(-1)=0 .
$$

Die Division ist mit der Argumentation gerechtfertigt, daß die identisch verschwindende Funktion nicht Eigenfunktion sein kann. Den Fall homogener Randbedingungen,

$$
r_{-1}(X)=a_{1} X(-1)+a_{2} X^{\prime}(-1)=0 \quad \text { bzw. } \quad r_{1}(X)=b_{1} X(1)+b_{2} X^{\prime}(1)=0,
$$

am linken bzw. rechten Intervallrand behandelt man für die erste der beiden Eigenwertaufgaben durch einen Ansatz der Form

$$
\begin{array}{lll}
\Phi(x, \mu)=C_{11} e^{i x \sqrt{\mu}}+C_{12} e^{-i x \sqrt{\mu}}, & \Phi(-1, \mu)=-a_{2}=1, & \Phi^{\prime}(-1, \mu)=a_{1}=0 \\
\Psi(x, \mu)=C_{21} e^{i x \sqrt{\mu}}+C_{22} e^{-i x \sqrt{\mu}}, & \Psi(1, \mu)=-b_{2}=-1, & \Psi^{\prime}(1, \mu)=b_{1}=0,
\end{array}
$$

wobei man sich leicht überzeugt, daß die beiden Funktionen $r_{-1}(\Phi)=0$ und $r_{1}(\Psi)=0$ erfüllen. Aus diesen Informationen können die Koeffizienten $C_{i j}$ berechnet werden und man findet

$$
\Phi(x, \mu)=\frac{1}{2} e^{\sqrt{\mu}} e^{i x \sqrt{\mu}}+\frac{1}{2} e^{-\sqrt{\mu}} e^{-i x \sqrt{\mu}}, \quad \Psi(x, \mu)=-\frac{1}{2} e^{-\sqrt{\mu}} e^{i x \sqrt{\mu}}-\frac{1}{2} e^{\sqrt{\mu}} e^{-i x \sqrt{\mu}} .
$$

Die Eigenwerte und Eigenfunktionen des Problems kommen mit Hilfe der folgenden Argumentation: Sind für einen festen Wert $\mu_{0}$ die beiden Funktionen $\Phi\left(x, \mu_{0}\right)$ und $\Psi\left(x, \mu_{0}\right)$ linear abhängig, so ist wegen der linearen Strukturen auch $r_{-1}(\Psi)=0$ und $r_{1}(\Phi)=0$, sodaß beide Funktionen die homogenen Randbedingungen an beiden Intervallgrenzen erfüllen und es sich um Eigenfunktionen zum Eigenwert $\mu_{0}$ handeln muß. Ein Kriterium zur Überprüfung der linearen Abhängigkeit der beiden Funktionen $\Phi$ und $\Psi$ ist das Verschwinden ihrer Wronskischen Determinante [7, S.883f.]. Somit bestimmt man nach kurzer Rechnung die Eigenwerte des ersten Problems durch Lösen von

$$
0=\mathfrak{W}(x ; \Phi(x, \mu), \Psi(x, \mu))=\frac{i \sqrt{\mu}}{2}\left[e^{2 i \sqrt{\mu}}-e^{-2 i \sqrt{\mu}}\right]=i \sqrt{\mu} \sinh 2 i \sqrt{\mu} \Longleftrightarrow e^{4 i \sqrt{\mu}}=1
$$


unter Beachtung von $\ln z=\ln |z|+i(\arg z+2 k \pi) \mathrm{zu}$

$$
\mu_{k}=\frac{k^{2} \pi^{2}}{4}, \quad k=1,2 \ldots
$$

Für die y-Richtung macht man in analoger Weise den Ansatz

$$
\begin{array}{lll}
\Phi(y, \nu)=C_{11} e^{i y \sqrt{\nu}}+C_{12} e^{-i y \sqrt{\nu}}, & \Phi(-1, \nu)=-a_{2}=1, & \Phi^{\prime}(-1, \nu)=a_{1}=\gamma \\
\Psi(y, \nu)=C_{21} e^{i y \sqrt{\nu}}+C_{22} e^{-i y \sqrt{\nu}}, & \Psi(1, \nu)=-b_{2}=-1, & \Psi^{\prime}(1, \nu)=b_{1}=0,
\end{array}
$$

wodurch man die Koeffizienten $C_{i j}$ berechnet und auf

$$
\begin{aligned}
& \Phi(y, \nu)=\frac{1}{2} e^{i \sqrt{\nu}}\left(1+\frac{\gamma}{i \sqrt{\nu}}\right) e^{i y \sqrt{\nu}}+\frac{1}{2} e^{-i \sqrt{\nu}}\left(1-\frac{\gamma}{i \sqrt{\nu}}\right) e^{-i y \sqrt{\nu}} \\
& \Psi(y, \nu)=-\frac{1}{2} e^{-i \sqrt{\nu}} e^{i y \sqrt{\nu}}-\frac{1}{2} e^{i \sqrt{\nu}} e^{-i y \sqrt{\nu}}
\end{aligned}
$$

geführt wird. Daraus resultiert die Eigenwertgleichung

$$
0=\mathfrak{W}(y ; \Phi(y, \nu), \Psi(y, \nu))=i \sqrt{\nu} \sinh 2 i \sqrt{\nu}+\gamma \cosh 2 i \sqrt{\nu} .
$$

Um diese nichtlineare komplexe Gleichung für eine numerische Lösung aufzubereiten, setzt man zweckmäßigerweise $2 \sqrt{\nu}=\xi+i \eta$. Nun ist aus der Theorie bekannt, daß sämtliche Eigenwerte des Problems einfach und reell sind, wodurch ein rein imaginärer beziehungsweise rein reeller Ansatz gerechtfertigt ist und obige Gleichung in die beiden reellen Gleichungen

$$
\begin{aligned}
\frac{i \xi}{2} \sinh i \xi+\gamma \cosh i \xi & =0 \Longleftrightarrow \tan \xi=\frac{2 \gamma}{\xi} \\
-\frac{\eta}{2} \sinh (-\eta)+\gamma \cosh (-\eta)=0 & \Longleftrightarrow \tanh \eta=-\frac{2 \gamma}{\eta}
\end{aligned}
$$

zerfällt. Läßt man den Fall $\gamma=0$, der klarerweise auf die Eigenwerte aus (1.8) führt, außer acht, so liefert der reelle Ansatz für positives $\gamma$ symmetrische Lösungen $\pm \xi_{j}$, die etwa für $\gamma=1$ von Mathematica mit beliebiger Genauigkeit ${ }^{1}$ berechnet werden können $\left(\xi_{1} \approx 1.0769, \xi_{2} \approx 3.6436\right.$, $\left.\xi_{3} \approx 6.5783, \ldots\right)$. Für positives $\gamma$ werden, wie man sich auch an Hand von Abbildung 1.2 vor Augen führen kann, vom rein imaginären Ansatz keine weiteren Lösungen beigesteuert und damit sind die Eigenwerte des zweiten Eigenwertproblems durch

$$
\nu_{j}=\frac{\xi_{j}^{2}}{4}, \quad j=1,2 \ldots
$$

gefunden. Für $\gamma<0$ tritt zusätzlich zu den Lösungen des reellen Ansatzes ein negativer Eigenwert, der aus physikalischer Sicht für die Erwärmung der unteren Quadratseite verantwortlich ist. Für den Fall $\gamma=-1$ errechnet Mathematica die Lösungen $\eta_{0} \approx 2.0653, \xi_{1} \approx 2.4587, \xi_{2} \approx 5.9594, \ldots$, es ist also neben 1.10 auch noch der negative Eigenwert $\nu_{0}=-\eta_{0}^{2} / 4 \mathrm{zu}$ berücksichtigen. Hinsichtlich der erwähnten Sonderfälle $\mu=0$ bzw. $\nu=0$ gilt, daß eindeutige Lösbarkeit des Gesamtproblems nur dann gegeben ist, wenn höchstens einer dieser Fälle eintritt. Jedenfalls kommen nur lineare Lösungen der Form $A y+B$ in Frage. Zieht man die allgemeineren Randbedingungen in $y$-Richtung zur Bildung der Funktionen $\Phi$ und $\Psi$ heran, so stößt man auf die Determinante

$$
\mathfrak{W}(y ; \Phi(y, 0), \Psi(y, 0))=\left|\begin{array}{cc}
\gamma(y+1)+1 & -1 \\
\gamma & 0
\end{array}\right|=\gamma,
$$

die für nichtverschwindendes $\gamma$ keine lineare Abhängigkeit zwischen den Funktionen hervorzubringen vermag. Demgemäß scheidet $\nu=0$ als Eigenwert des zweiten Problems aus, es liegt eindeutige Lösbarkeit der Gleichung (1.1) vor. Wohl tritt aber der Fall $\mu=0$ ein, für den man leicht bestätigt, daß es sich bei den zugehörigen Lösungsfunktionen sogar um konstante Funktionen handelt.

${ }^{1} f\left[x_{-}\right]:=x * \operatorname{Tan}[x]-2 ; \operatorname{For}[i=1, i \leq 20, i++, N S T[[i]]=F i n d R o o t[f(x),\{x, 3.14 i-2.6\}$, WorkingPrecision $\rightarrow 500]]$; 

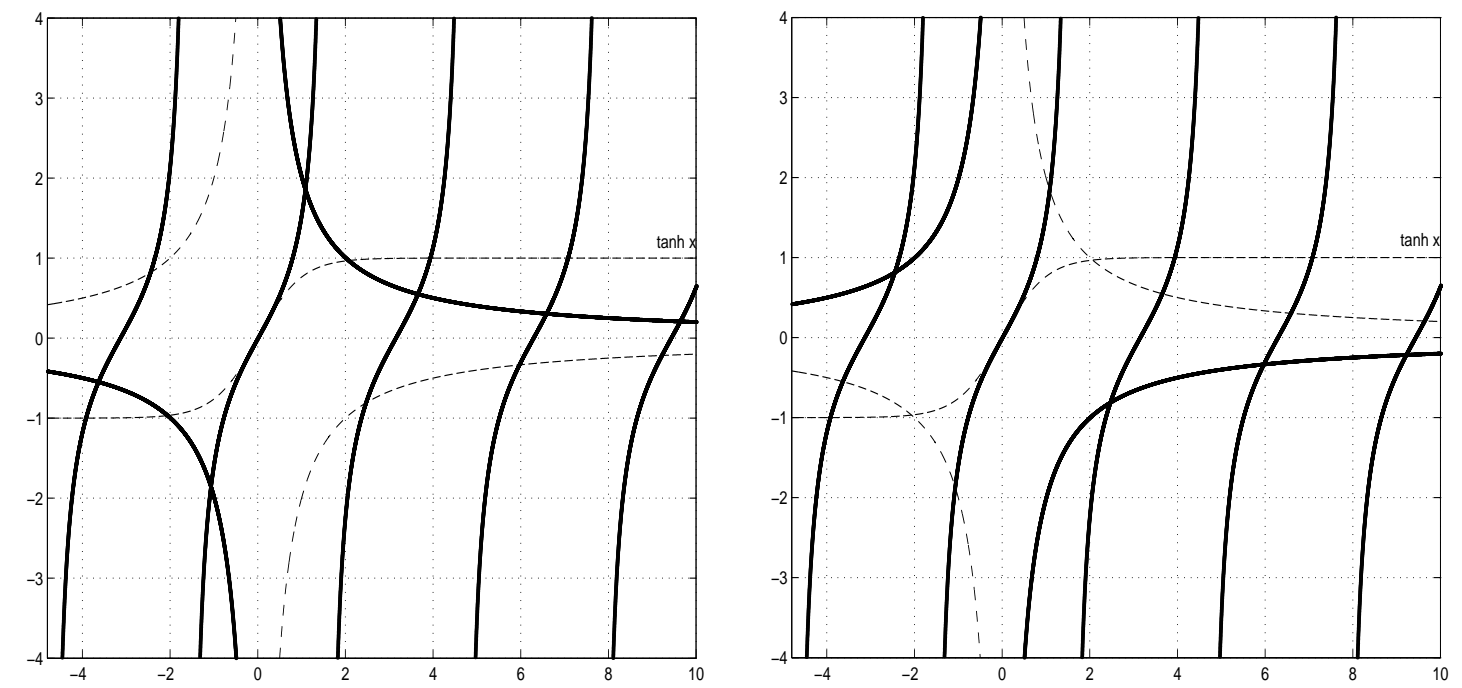

Abbildung 1.2: Für positives $\gamma=1$ (links) liefert der reelle Ansatz keine weiteren negativen Eigenwerte, während für negatives $\gamma=-1$ (rechts) aus dem reellen Ansatz zusätzlich ein negativer Eigenwert resultiert, der für unbeschränktes Wachstum verantwortlich ist.

Wir berechnen jetzt die Eigenfunktionen, von denen aus der Theorie bekannt ist, daß das innere Produkt zweier Eigenfunktionen zu zwei verschiedenen Eigenwerten bezüglich der quadratischen Integralnorm verschwindet. Zusätzlich zu dieser Orthogonalitätseigenschaft ist es zweckmäßig, die Eigenfunktionen im Sinne von $\int_{-1}^{1} X_{i}(x) X_{j}(x) d x=\delta_{i j}$ zu normieren. Auf Grund ihrer linearen Abhängigkeit erfüllen die Funktionen aus (1.7) für jeden Eigenwert die Beziehung $\Psi\left(x, \mu_{k}\right)=c_{k} \Phi\left(x, \mu_{k}\right)$, und bezeichnet man mit $\omega^{\prime}$ die Ableitung der WronsKischen Determinante nach dem Parameter $\mu$, so führt die Setzung $X_{k}(x):=\sqrt{c_{k} / \omega^{\prime}\left(\mu_{k}\right)} \Phi\left(x, \mu_{k}\right), k=1,2 \ldots$, zu den normierten Eigenfunktionen. Für die $x$-Koordinate bedeutet dies

$$
X_{0}(x)=\frac{\sqrt{2}}{2}, \quad X_{k}(x)=\cos \frac{k \pi(x+1)}{2}, \quad k=1,2 \ldots .
$$

Für die y-Richtung bestreitet man eine längere Rechnung, bei der man unter Ausnutzung des Verschwindens der WrONSKIschen Determinante und einiger trigonometrischer Identitäten zu einer für beliebiges $\gamma$ gültigen Darstellung der Eigenfunktionen ${ }^{2}$ in Form von

$$
Y_{j}(y)=\frac{1}{\sqrt{1+\frac{2 \gamma}{4 \gamma^{2}+\xi_{j}^{2}}}} \cos \frac{\xi_{j}(y-1)}{2}, \quad j=1,2 \ldots
$$

gelangt. Für negatives $\gamma$ errechnet man zusätzlich die Eigenfunktion

$$
Y_{0}(y)=\frac{1}{\sqrt{1+\frac{2 \gamma}{4 \gamma^{2}-\eta_{0}^{2}}}} \cosh \frac{\eta_{0}(y-1)}{2} .
$$

Es besagt nun der Entwicklungssatz nach Eigenfunktionen in seiner eindimensionalen Fassung [7. S.1154], daß sich jede auf einem abgeschlossenen Intervall stückweise stetige Funktion in ihre Orthogonalreihe nach den Eigenfunktionen des zugehörigen STURM-LiouviLLEschen Differentialoperators entwickeln läßt, so dieser auf dem offenen Intervall regulär ist. Mit der Regularität werden dabei gewisse Stetigkeitsforderungen an die Koeffizientenfunktionen des Differentialoperators verbunden, die im vorliegenden Fall des Laplace-Operators erfüllt sind. Für beliebiges, aber festes $\hat{y}$

\footnotetext{
${ }^{2}$ Im Rechengang wird der kompakteren Darstellung mit Hilfe der Funktionen $\Psi\left(y, \nu_{j}\right)$ der Vorzug gegeben. Wegen der Proportionalität $Y_{j}(y)=\sqrt{\frac{c_{j}}{\omega^{\prime}\left(\nu_{j}\right)}} \frac{\Psi\left(y, \nu_{j}\right)}{c_{j}}$ kann das Auftreten von Sinustermen umgangen werden.
} 
aus dem Intervall $(-1,1)$ ist damit eine Entwicklung nach den Eigenfunktionen $X_{k}(x)$ in Form von $u(x, \hat{y})=\sum_{k=0}^{\infty} X_{k}(x) \int_{-1}^{1} X_{k}(\xi) u(\xi, \hat{y}) d \xi$ möglich.

Läßt man jetzt $\hat{y}$ variieren, so wird das in der Summe auftretende Integral eine von $y$ allein abhängige Funktion, $c_{k}(y)$, die auf dem offenen Intervall $(-1,1)$ in ihre Eigenfunktionen $Y_{j}(y)$ entwickelt werden kann: $c_{k}(y)=\sum_{j=0}^{\infty} Y_{j}(y) \int_{-1}^{1} Y_{j}(\eta) c_{k}(\eta) d \eta$. Für $\gamma>0$ beginnt die Summation entsprechend mit $j=1$, für verschwindendes $\gamma$ ist die Eigenfunktion $Y_{0}(y)$ durch eine geeignete Normierungskonstante zu ersetzen. Durch Einsetzen der Entwicklung von $c_{k}(y)$ in jene von $u(x, \hat{y})$ hat man also einen Entwicklungssatz zur Hand, der es gestattet, beliebige auf $\mathfrak{Q}$ stückweise stetige Funktionen zweier Veränderlicher mit Hilfe der gefundenen Eigenfunktionen darzustellen. Insbesondere gilt dies für die Funktion $\triangle u$, die auch noch von einem Parameter $t$ abhängig sein kann. Man gelangt unter Beachtung von $u_{t} / \kappa=\triangle u=\lambda u$ und der entsprechenden Entwicklung der gesuchten Funktion $u$ zur Darstellung

$$
\triangle u(x, y, t)=\sum_{k=0}^{\infty} \sum_{j=0}^{\infty} X_{k}(x) Y_{j}(y) \lambda_{k j} \int_{-1}^{1} \int_{-1}^{1} X_{k}(\xi) Y_{j}(\eta) u(\xi, \eta, t) d \xi d \eta
$$

Mit einer der obigen analogen Überlegung kann das Doppelintegral als eine vom Zeitparameter allein abhängige Funktion, $c_{k j}(t)$, aufgefaßt werden. Durch Ersetzen des Laplace-Operators durch die Zeitableitung findet sich ein nicht gekoppeltes Differentialgleichungssystem $\dot{c}_{k j}(t)=\kappa \lambda_{k j} c_{k j}(t)$, also $c_{k j}(t)=\alpha_{k j} e^{\kappa \lambda_{k j} t}$. Zur eindeutigen Lösung dieser reinen Anfangswertprobleme zieht man die Anfangsbedingung $u(x, y, 0)=1$ des Gesamtproblems heran ${ }^{3}$, wobei im Falle $\gamma=0$ für $j>0$ sämtliche $\alpha_{0 j}$ verschwinden, man findet $\alpha_{00}=2=c_{00}(t)$, letzteres wegen $\lambda_{00}=-\mu_{0}-\nu_{0}=0$. Die Lösung ist hier konstant, $u(x, y, t) \equiv 1$. Für $\gamma>0$ ist $j>0$ und das Einsetzen der Randbedingung führt ${ }^{4}$ schließlich zur Reihendarstellung der gesuchten Lösungsfunktion,

$$
u(x, y, t)=\sum_{j=1}^{\infty} \frac{2}{\xi_{j}} \frac{\sin \xi_{j}}{1+\frac{2 \gamma}{4 \gamma^{2}+\xi_{j}^{2}}} \cos \frac{\xi_{j}(y-1)}{2} e^{-\frac{\kappa \xi_{j}^{2}}{4} t} .
$$

Für $\gamma<0$ hat man noch dem negativen Eigenwert $\mu_{0}$ Rechnung zu tragen. Mit ähnlichem Rechengang bestimmt man $\alpha_{00}=c_{00}(t)$ und findet einen der obigen Summe hinzuzufügenden Term,

$$
\frac{2}{\eta_{0}} \frac{\sinh \eta_{0}}{1+\frac{2 \gamma}{4 \gamma^{2}-\eta_{0}^{2}}} \cosh \frac{\eta_{0}(y-1)}{2} e^{\frac{\kappa \eta_{0}^{2}}{4} t}
$$

der das exponentielle Wachstum im Sinne einer Zuführung von Wärme ad infinitum widerspiegelt, da dann eine Erwärmung an der unteren Quadratberandung proportional zum Temperaturgradienten der dort vorherrschenden Temperatur erfolgt. Daß die vorliegende Reihenlösung die Ausgangsforderung erfüllt, kann man unmittelbar durch Ausdifferenzieren und Einsetzten in (1.1) nachprüfen.

Abbildung 1.3 zeigt die Lösungsverhältnisse für $\gamma=\kappa=1$. Zur Reihenkonvergenz wird bemerkt, daß für $t>0$ mit fortschreitender Zeit dem Exponentialterm eine zunehmend dominante Bedeutung zufällt. So ist für $t=1$ bereits die Verwendung von sieben Summanden ausreichend, um eine Genauigkeit von 50 Nachkommastellen auf dem abgeschlossenen Einheitsquadrat sicherzustellen. Für $t=0$ entfällt der Exponentialterm und die Lösungsfunktion oszilliert bei der Approximation der Anfangsbedingung. Insbesondere ist die Konvergenz der Reihenentwicklung um jene Punkte, in denen die Newtonsche Randbedingung zu gelten hat, sehr langsam. Diese Umstände sind aber für die weiteren Untersuchungen weniger von Bedeutung, da wir primär an einer Referenzlösung von großer Güte für $t>0$ interessiert sind, die mit Simulationslösungen verglichen werden soll.

\footnotetext{
${ }^{3}$ Für den etwas allgemeineren Fall einer von $x$ unabhängigen Anfangsbedingung der Form $u(x, y, 0)=f(y)$ kann gezeigt werden, daß auch die gesuchte Lösungsfunktion unabhängig von der Variablen $x$ sein muß.

${ }^{4}$ über $\alpha_{0 j}=\int_{-1}^{1} \frac{\sqrt{2}}{2} d \xi \int_{-1}^{1}\left(\cos \frac{\xi_{j}(\eta-1)}{2}\right) / \sqrt{1+2 \gamma /\left(4 \gamma^{2}+\xi_{j}^{2}\right)} d \eta=\left(2 \sqrt{2} \sin \xi_{j}\right) /\left(\xi_{j} \sqrt{1+2 \gamma /\left(4 \gamma^{2}+\xi_{j}^{2}\right)}\right)$
} 

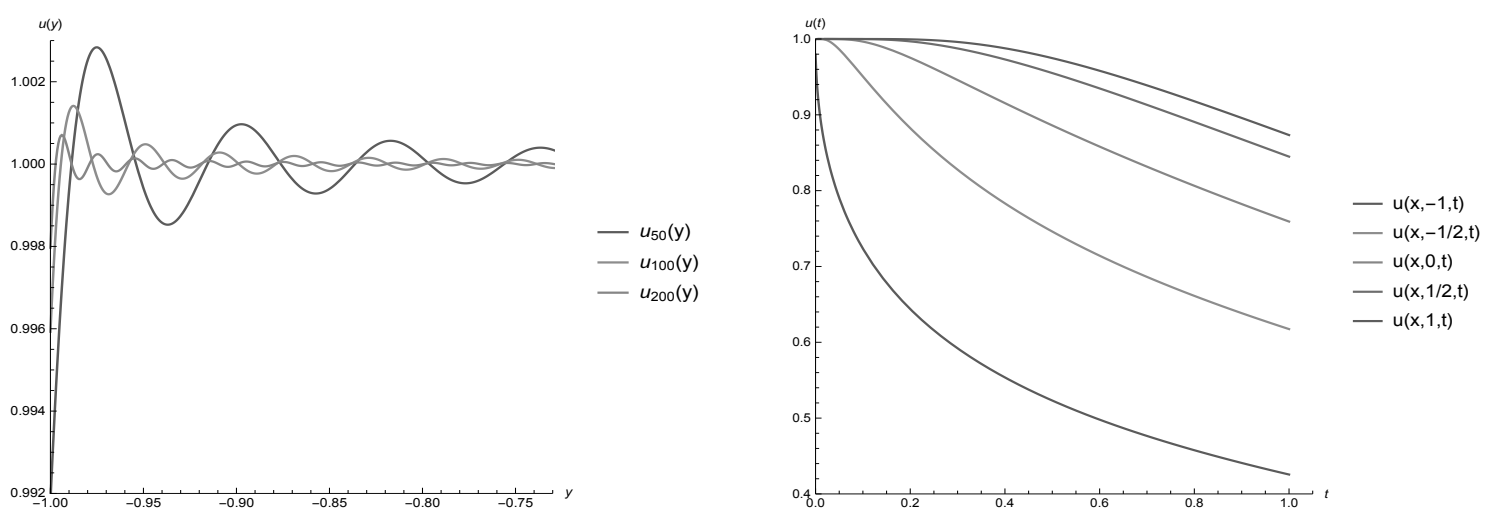

Abbildung 1.3: Die Lösung 1.11 des Wärmeleitungsproblems 1.1 in der Nähe der NEWTonschen Randbedingung mit $j=50, j=100$ und $j=200$ Summanden für $t=0$ (links) und im Zeitintervall $[0,1]$ in den Punkten $y=-1,-\frac{1}{2}, 0, \frac{1}{2}, 1$ unter Verwendung von $j=20$ Summanden (rechts).

\subsection{Lösung des Problems mit der CTDS-Linienmethode}

In den wenigsten Fällen wird es möglich sein, für ein gegebenes Anfangsrandwertproblem zu einer Lösung in geschlossener Form zu gelangen. Es liegt daher nahe, nach Näherungslösungen mittels Computersimulation zu suchen. Ein wesentlicher Aspekt bei einer solchen Vorgehensweise ist der Umstand, daß in der dabei zu Grunde liegenden Computerarithmetik nur ein endlicher Zahlenbereich zur Verfügung steht und somit zwingend der Übergang vom ursprünglichen, kontinuierlichen Problem zu einer diskreten Aufgabenstellung notwendig wird. Genau genommen wird dieser Umstand bereits bei der Auswertung der Lösungsfunktion (1.11) schlagend: Die Genauigkeit der (irrationalen) Nullstellen von Gleichung (1.9) unterliegt dabei ebenso der Beschränkung der Unmöglichkeit einer exakten Darstellung mittels Maschinenzahlen, wie auch die Reihenentwicklung selbst nach endlich vielen Summanden abbrechen muss. Mithin ist das in Abbildung 1.3 zu beobachtende Über- und Unterschwingen damit zu begründen und somit numerischer Natur.

Ein möglicher Ansatz zur Behandlung partieller Differentialgleichungen auf Rechenanlagen ersetzt die in den Gleichungen auftretenden Ableitungen durch deren diskrete Äquivalente, den Finiten Differenzenquotienten. Hinsichtlich Genauigkeit und Flexibilität wird diese Methode der Finiten Differenzen zwischen der Methode der Finiten Elemente und den Spektralmethoden angesiedelt. Ihre Stärken zeigen sich, wenn die Koordinatenlinien mit den Richtungen der Ortsableitungen zusammenfallen, sind aber theoretisch auch in anderen Fällen anwendbar.

Die $\boldsymbol{C T D S}$-Linienmethode als ein Spezialfall der Methode der Finiten Differenzen ersetzt in den Punkten eines endlichen, regelmäßigen Rechengitters zunächst nur die auftretenden partiellen Differentialquotienten des Ortes durch die entsprechenden finiten Näherungsquotienten (,Discrete Space $^{\text {") }}$, um so im Zeitbereich zu einem gekoppelten System gewöhnlicher Differentialgleichungen zu gelangen („Continuous Time“). Dieses System wird dann mit Standardmethoden weiter behandelt. Der Grundgedanke ist bei Breitenecker in [4, S.341ff.] dargelegt.

Zur Lösung von Problem (1.1) - ohne die Allgemeinheit zu beschränken sei der Bequemlichkeit halber wieder $\gamma=\kappa=1$ gesetzt - und den zugehörigen Randbedingungen mittels CTDSLinienmethode definieren wir zunächst das für alles weitere fundamentale Rechengitter

$$
\mathfrak{Q}_{n_{1}, n_{2}}:=\left\{\left(x_{i}, y_{j}\right) \in \mathbb{R}^{2} \mid x_{i}=-1+\frac{2(i-1)}{n_{1}-1}, y_{j}=-1+\frac{2(j-1)}{n_{2}-1}, i=1, \ldots n_{1}, j=1, \ldots n_{2}\right\}
$$

als endliche Menge von geordneten Tupeln im Sinne einer Teilmenge des abgeschlossenen Einheitsquadrates $\overline{\mathfrak{Q}}$. Beachtet man unter Verwendung obiger Definitionen für $x_{i}$ und $y_{j}$ den Isomorphismus zwischen $\mathbb{R}^{2}$ und dem Körper der komplexen Zahlen $\mathbb{C}$, so erhält man die äquivalente Darstellung

$$
\mathfrak{Q}_{n_{1}, n_{2}}=\left\{z_{i j} \in \mathbb{C} \mid z_{i j}=x_{i}+i y_{j}, \Re z_{i j}=x_{i}, \Im z_{i j}=y_{j}, i=1, \ldots n_{1}, j=1, \ldots n_{2}\right\} .
$$


Der als in die jeweilige Koordinatenrichtung äquidistant definierte Abstand zwischen den $n_{1} \times n_{2}$ Gitterpunkten entspricht dabei den Differenzen

$$
\begin{array}{ll}
\Delta_{x}=x_{i}-x_{i-1}=\frac{2}{n_{1}-1}, & i=2, \ldots n_{1} \quad \text { bzw. } \\
\Delta_{y}=y_{j}-y_{j-1}=\frac{2}{n_{2}-1}, \quad j=2, \ldots n_{2} .
\end{array}
$$

Die Verwendung eines zwischen den Gitterpunkten konstanten Abstandes führt zu erheblichen Vereinfachungen hinsichtlich der Gestalt der Differenzenquotienten, ferner kann dadurch eine höhere Fehlerordnung bei der Approximation der gesuchten Ableitungen erzielt werden. Nicht äquidistante, regelmäßige Rechengitter können hingegen in jenen Fällen in Erwägung gezogen werden, in denen es notwendig ist, bei Existenz von Bereichen mit betragsmäßig hohen Gradienten dort mit einer lokal feineren Auflösung zu rechnen. Auch ist eine adaptive Zuschaltung zusätzlicher Gitterpunkte bei Auftreten von Zustandsereignissen (etwa im Falle von Unstetigkeiten) denkbar.

An die Stelle der auf $\mathfrak{Q}$ bzw. unter Berücksichtigung der Randbedingungen auf $\overline{\mathfrak{Q}}$ gesuchten Funktion $u(x, y, t)$ treten nun $n_{1} \times n_{2}$ ausschließlich von der Zeit abhängige Funktionen $u_{i, j}(t)$, die nur im jeweiligen Punkt $\left(x_{i}, y_{j}\right)$ des Rechengitters $\mathfrak{Q}_{n_{1}, n_{2}}$ definiert sind und denen zur Bildung der lokalen Ortsableitungen Informationen aus den benachbarten Gitterpunkten zufließen. Zum Auffinden geeigneter Approximationen untersucht man die Taylorreihenentwicklung der $(n+1)$-mal stetig differenzierbaren Funktion $u(x, y, t)$ um den Gitterpunkt $\left(x_{i}, y_{j}\right)$, es gilt in positive $x$-Richtung unter Verwendung des Restgliedes von LAGRANGE mit $0<\tau<1$ die Entwicklung

$$
u\left(x_{i}+\Delta_{x}, y, t\right)=\sum_{k=0}^{\infty} \frac{\partial u^{(k)}\left(x_{i}, y, t\right)}{\partial x^{k}} \frac{\Delta_{x}^{k}}{k !}=\sum_{k=0}^{n} \frac{\partial u^{(k)}\left(x_{i}, y, t\right)}{\partial x^{k}} \frac{\Delta_{x}^{k}}{k !}+\frac{\partial u^{(n+1)}\left(x_{i}+\tau \Delta_{x}, y, t\right)}{\partial x^{n+1}} \frac{\Delta_{x}^{n+1}}{(n+1) !} .
$$

Mit der naheliegenden Forderung nach $u_{i, j}(t)=u\left(x_{i}, y_{j}, t\right)$, daß also in den Gitterpunkten die Funktionen des Rechengitters mit der Lösungsfunktion auf dem Einheitsquadrat übereinstimmen sollen, findet man unter Verwendung der LANDAUschen Symbolik und dem Übergang $\Delta_{x} \rightarrow-\Delta_{x}$ die beiden Entwicklungen

$$
\begin{aligned}
& u\left(x_{i}+\Delta_{x}, y, t\right)=u\left(x_{i}, y, t\right)+\frac{\partial u\left(x_{i}, y, t\right)}{\partial x} \Delta_{x}+\frac{\partial^{2} u\left(x_{i}, y, t\right)}{\partial x^{2}} \frac{\Delta_{x}^{2}}{2}+\frac{\partial^{3} u\left(x_{i}, y, t\right)}{\partial x^{3}} \frac{\Delta_{x}^{3}}{6}+\mathcal{O}\left(\Delta_{x}^{4}\right) \\
& u\left(x_{i}-\Delta_{x}, y, t\right)=u\left(x_{i}, y, t\right)-\frac{\partial u\left(x_{i}, y, t\right)}{\partial x} \Delta_{x}+\frac{\partial^{2} u\left(x_{i}, y, t\right)}{\partial x^{2}} \frac{\Delta_{x}^{2}}{2}-\frac{\partial^{3} u\left(x_{i}, y, t\right)}{\partial x^{3}} \frac{\Delta_{x}^{3}}{6}+\mathcal{O}\left(\Delta_{x}^{4}\right),
\end{aligned}
$$

aus deren Addition man nach Umformung einen Ausdruck für die zweite Ortsableitung erhält, der bei Verfeinerung des Abstandes $\Delta_{x}$ mit einer Genauigkeit von zweiter Ordnung gegen Null strebt:

$$
\frac{\partial^{2} u\left(x_{i}, y_{j}, t\right)}{\partial x^{2}}=\frac{u_{i+1, j}(t)-2 u_{i, j}(t)+u_{i-1, j}(t)}{\Delta_{x}^{2}}+\mathcal{O}\left(\Delta_{x}^{2}\right)
$$

Da man einen ähnlichen Ausdruck in völlig analoger Weise auch für die zweite partielle Ableitung in $y$-Richtung herleitet, besteht für $2 \leq i \leq n_{1}-1$ und $2 \leq j \leq n_{2}-1$ die Möglichkeit, den in Gleichung (1.1) auftretenden LAPLACEschen Operator durch die entsprechenden Näherungsausdrücke zu ersetzen. Für den Fall $n_{1}=n_{2}=n$ und somit $\Delta_{x}=\Delta_{y}=\Delta$ führt dies auf ein gekoppeltes System von $(n-2) \times(n-2)$ gewöhnlichen Differentialgleichungen der Form

$$
\dot{u}_{i, j}(t)=\frac{u_{i+1, j}(t)+u_{i, j+1}(t)-4 u_{i, j}(t)+u_{i-1, j}(t)+u_{i, j-1}(t)}{\Delta^{2}}, \quad i, j=2 \ldots, n-1 .
$$

Die an das System zu stellenden Anfangsbedingungen können vom kontinuierlichen Ausgangsproblem 1.2 unverändert übernommen werden, womit die Fragestellung nach den Verhältnissen im Inneren des Einheitsquadrates zufriedenstellend geklärt ist. Damit die Simulation des Problems mit Hilfe eines Computerprogrammes ermöglicht werden kann, ist der einzige jetzt noch offene Aspekt jener der Behandlung der Randbedingungen, es müssen folglich die Funktionswerte in den 
Randgitterpunkten in Beziehung zu jenen in den benachbarten inneren Punkten gebracht werden. Betrachtet man dazu im einfachsten Fall das TAYLORsche Polynom ersten Grades, so findet man für $i=1$ bzw. $i=n$ und $j=2, \ldots n-1$ die Vorwärts- und Rückwärtsdifferenzen

$$
\frac{\partial u\left(x_{1}, y_{j}, t\right)}{\partial x}=\frac{u_{2, j}(t)-u_{1, j}(t)}{\Delta}+\mathcal{O}(\Delta) \quad \text { bzw. } \quad \frac{\partial u\left(x_{n}, y_{j}, t\right)}{\partial x}=\frac{u_{n, j}(t)-u_{n-1, j}(t)}{\Delta}+\mathcal{O}(\Delta) .
$$

Auf der Berandung von $\mathfrak{Q}_{n, n}$ treten unter spezieller Beachtung von $\nabla_{\mathbf{n}} u(x,-1, t)=-u_{y}(x,-1, t)$ für den unteren Quadratrand demnach noch die Bedingungen

$$
\begin{aligned}
u_{n, j}(t) & =u_{n-1, j}(t), & j & =2, \ldots n-1 \\
u_{1, j}(t) & =u_{2, j}(t), & j & =2, \ldots n-1 \\
u_{i, n}(t) & =u_{i, n-1}(t), & i & =2, \ldots n-1 \\
u_{i, 1}(t) & =\frac{u_{i, 2}(t)}{1+\Delta}, & i & =2, \ldots n-1
\end{aligned}
$$

an die Seite der Gleichungen 1.12. Für die Implementierung der NEumannschen Randbedingungen (1.3)-1.5 genügt es im vorliegenden Fall der Verwendung von elementaren Vorwärts- und Rückwärtsdifferenzen (1.13), die Temperaturen in den Randpunkten den Temperaturen in den nächstgelegenen inneren Punkten gleichzusetzen. Dies ist aber für die Rechenpraxis auf Grund der nur linearen Fehlerordnung ein kaum gangbarer Weg. Im Zuge der systematischen Entwicklung eines Computerprogrammes zur Simulation wurden obige Gleichungen nicht zuletzt deshalb ebenfalls implementiert, um eine weitere Vergleichsmöglichkeit hinsichtlich der Verfahrensgenauigkeit sowie der korrekten Realisierung des Programmes zur Hand zu haben. Ein solches Ergebnis zeigt Abb. 1.4.
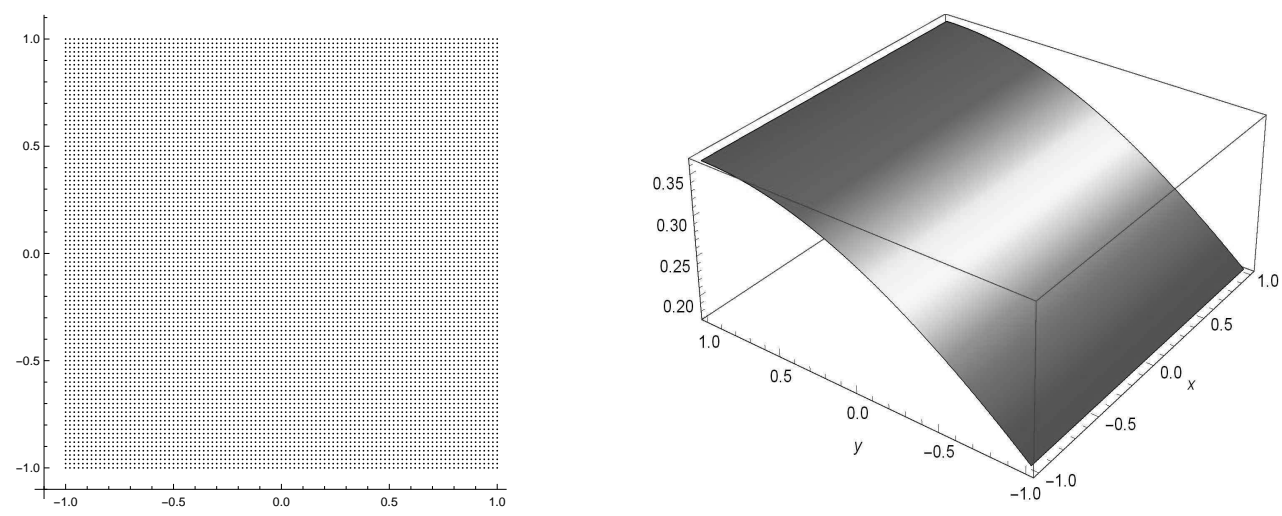

Abbildung 1.4: Das Rechengitter $\mathfrak{Q}_{101,101}$ mit $\Delta x=\Delta y=0.02$ (links) und die Simulationslösung von 1.12, 1.14 - 1.17) auf $\mathfrak{Q}_{101,101}$ zum Zeitpunkt $t=4$ (rechts).

Wir bemerken noch, daß auf die Funktionswerte in den Eckpunkten des Rechengitters zu keinem Zeitpunkt zugegriffen werden muss. Dies ist deshalb von Bedeutung, weil bei einer später noch durchzuführenden konformen Transformation von $\mathfrak{Q}_{n, n}$ genau an diesen Stellen Singularitäten zu erwarten sind. Solche Singularitäten würden allerdings gemäß ihrer Natur jedes numerische Verfahren vor große Probleme stellen.

Zur Steigerung der Genauigkeit kann man nun danach trachten, Näherungsformeln für die Ortsableitungen mit Hilfe des TAYLORschen Polynoms zu finden, dessen Restglied die entsprechende Ordnung aufweist. Diese Untersuchungen erweisen sich als mühevoll, weshalb alternativ dazu in Abschnitt 1.5 eine Methode präsentiert wird, mit deren Hilfe man Näherungsausdrücke von (theoretisch) beliebiger Genauigkeit für partielle Ableitungen beliebiger Ordnung findet.

Bei Schiesser [29, S.112f.] liest man für die zweite partielle Ableitung in $x$-Richtung jeweils für $i=3, \ldots n-2$ eine brauchbare äquidistante Zentraldifferenz von vierter Ordnung nach, wobei durch die Verwendung der Funktionswerte in fünf Gitterpunkten eine gesonderte Untersuchung der 
nächstinneren Randpunkte $(i=2$ und $i=n-1)$ notwendig wird. In diesen Punkten müssen zur Erzielung der selben Genauigkeit jedoch sechs Stützpunkte herangezogen werden (die Formeln dafür werden ebendort präsentiert), sodaß bei einem Fehler von $\mathcal{O}\left(\Delta^{4}\right)$ die Näherung

$$
\frac{\partial^{2} u\left(x_{i}, y_{j}, t\right)}{\partial x^{2}} \approx \begin{cases}\frac{-u_{i+2, j}(t)+16 u_{i+1, j}(t)-30 u_{i, j}(t)+16 u_{i-1, j}(t)-u_{i-2, j}(t)}{12 \Delta^{2}}, & i=3 \ldots n-2 \\ \frac{u_{6, j}(t)-6 u_{5, j}(t)+14 u_{4, j}(t)-4 u_{3, j}(t)-15 u_{2, j}(t)+10 u_{1, j}(t)}{12 \Delta^{2}}, & i=2 \\ \frac{u_{n-5, j}(t)-6 u_{n-4, j}(t)+14 u_{n-3, j}(t)-4 u_{n-2, j}(t)-15 u_{n-1, j}(t)+10 u_{n, j}(t)}{12 \Delta^{2}}, & i=n-1\end{cases}
$$

verwendet werden kann. Auch bei Ferziger und Perić ([11, S.47ff.]) findet man diese zweite zentrale Differenz, teilweise werden dort zusätzlich auch Entwicklungen für nicht-äquidistante Gitter untersucht. Die zweite partielle Ableitung in $y$-Richtung diskretisiert man in völlig analoger Weise und erhält damit für den LAPLACEschen Operator ein diskretes Äquivalent, mit dessen Hilfe man sich wieder ein System von $(n-2) \times(n-2)$ gekoppelten gewöhnlichen Differentialgleichungen konstruiert, das an die Stelle von (1.12) tritt.

Hinsichtlich der Randbedingungen ist es zweckmäßig nach Ausdrücken zu suchen, deren Fehler ebenso wie derjenige der Approximationen im Quadratinneren wie $\mathcal{O}\left(\Delta^{4}\right)$ gegen Null strebt, um eine einheitliche Vorgehensweise zu gewährleisten. Die Gleichungen (1.14)-(1.17) sind dann unter Verwendung von [29, S.108] zu ersetzen durch

$$
\begin{array}{lll}
u_{n, j}(t) & =\frac{48 u_{n-1, j}(t)-36 u_{n-2, j}(t)+16 u_{n-3, j}(t)-3 u_{n-4, j}(t)}{25}, & j=2, \ldots n-1 \\
u_{1, j}(t)=\frac{48 u_{2, j}(t)-36 u_{3, j}(t)+16 u_{4, j}(t)-3 u_{5, j}(t)}{25}, & j=2, \ldots n-1 \\
u_{i, n}(t)=\frac{48 u_{i, n-1}(t)-36 u_{i, n-2}(t)+16 u_{i, n-3}(t)-3 u_{i, n-4}(t)}{25}, & i=2, \ldots n-1 \\
u_{i, 1}(t)=\frac{48 u_{i, 2}(t)-36 u_{i, 3}(t)+16 u_{i, 4}(t)-3 u_{i, 5}(t)}{25+12 \Delta}, & i=2, \ldots n-1 .
\end{array}
$$

Im gegenständlichen Fall sind also jeweils vier innere Gitterpunkte zur Bildung der Ableitung in Normalenrichtung heranzuziehen. Damit sind sämtliche Bedingungen formuliert und das System kann simuliert und anschließend mit der mittels Reihenansatz gewonnenen Lösung verglichen werden. Der nachfolgende Code soll die prinzipielle Vorgehensweise der Implementierung an Hand von 1.12, 1.14-1.17) aufzeigen, die Ortsdiskretisierungen können bei komplizierteren Ausdrücken auch in einer externen Datei definiert und vom Hauptprogramm eingelesen werden.

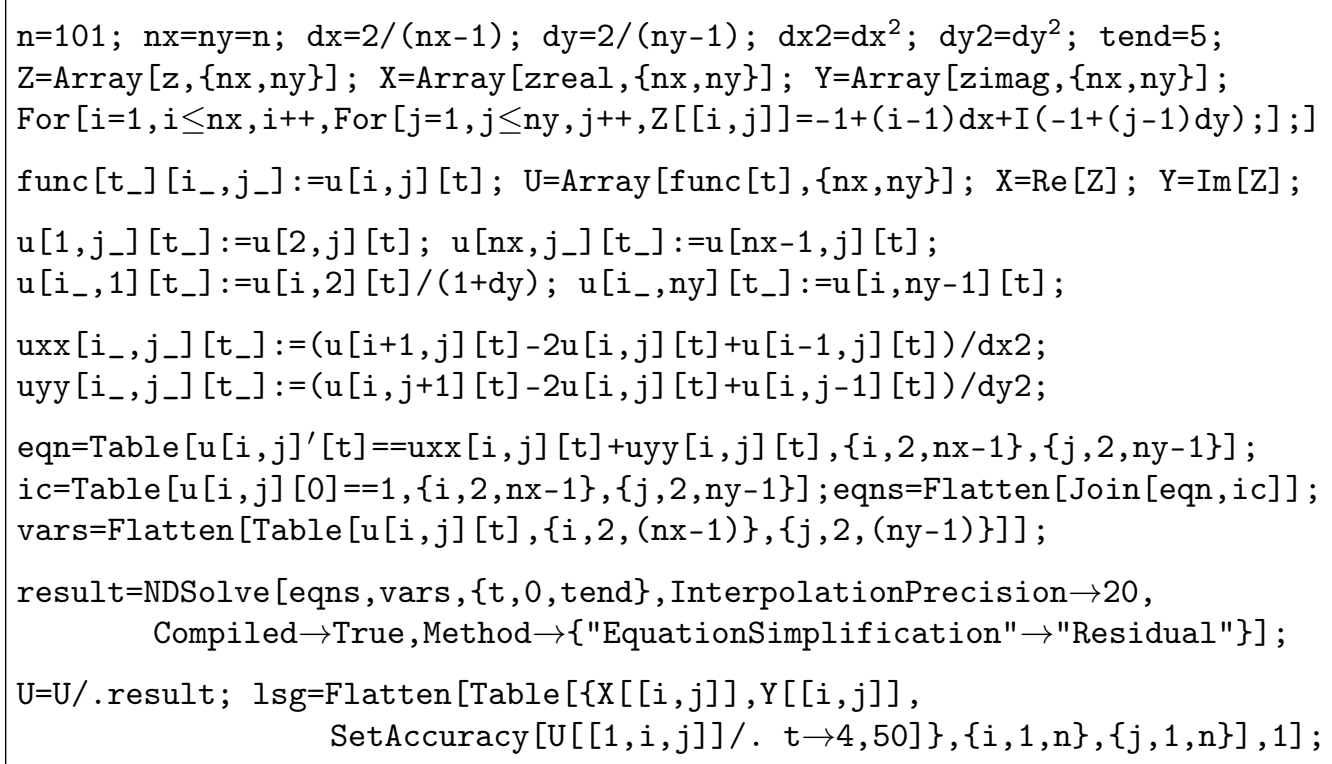




\subsection{Vergleich der Simulationslösungen mit dem Reihenansatz}

Abkürzend bezeichne $F D 1$ die Methode, die im Inneren nach der 5-Punkt-Formel (1.12) und am Rand mittels (1.14)-1.17) vorgeht, FD2 sei das Verfahren, das am Rand Diskretisierungsmethode 1.18-1.21 und im Inneren die 9-Punkt-Formeln (unter Berücksichtigung der nächstinneren Randpunkte) verwendet. Ferner sind unter PdeTbx die Ergebnisse der von Matlab verwendeten Methode der Finiten Elemente (der Partial Differential Equation Toolbox) zusammengestellt. Definiert man den relativen Fehler eines Verfahrens im Gitterpunkt $\left(x_{i}, y_{j}\right)$ zum Zeitpunkt $t$ bezüglich der dort bekannten exakten Lösung $\hat{u}\left(x_{i}, y_{j}, t\right)$ als Quotient

$$
e_{i, j}(t):=\frac{\hat{u}\left(x_{i}, y_{j}, t\right)-u\left(x_{i}, y_{j}, t\right)}{\hat{u}\left(x_{i}, y_{j}, t\right)},
$$

so können die Verfahren qualitativ wie quantitativ miteinander verglichen werden Abbildung 1.5. Es zeigt sich bereits hier, daß durch die Berücksichtigung mehrerer benachbarter Punkte im Zuge des Diskretisierungsprozesses eine extreme Senkung des relativen Fehlers möglich wird. Um das
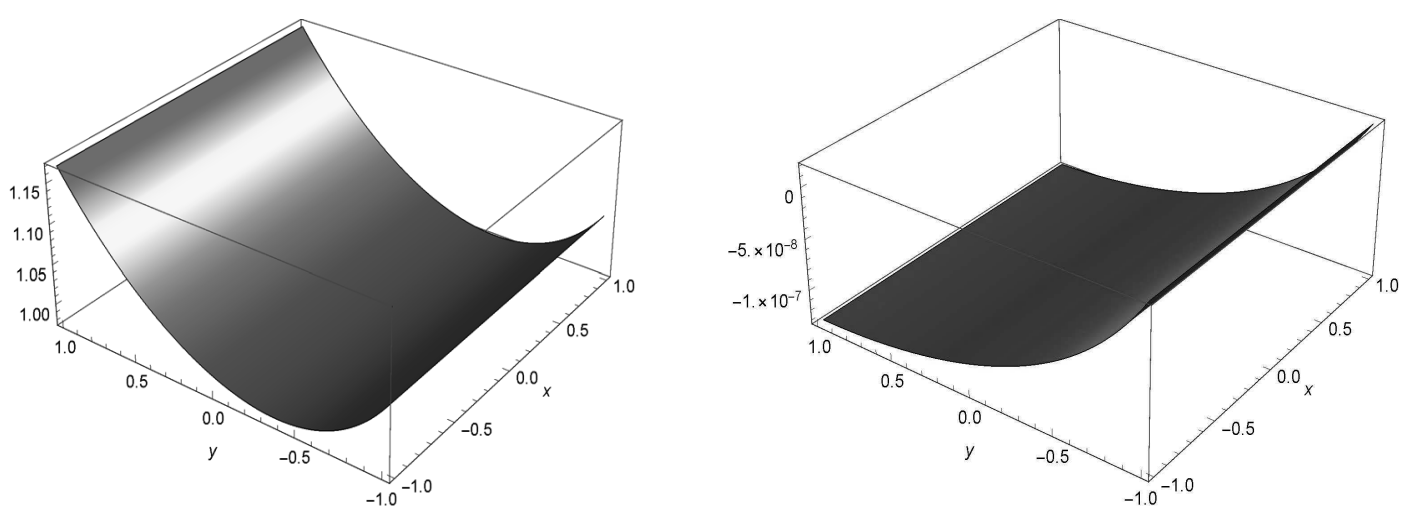

Abbildung 1.5: Die relativen Fehler von FD1 (links) und FD2 (rechts) in Bezug zur Reihenlösung (1.11) auf $\mathfrak{Q}_{101,101}$ zum Zeitpunkt $t=4$ in Prozent.
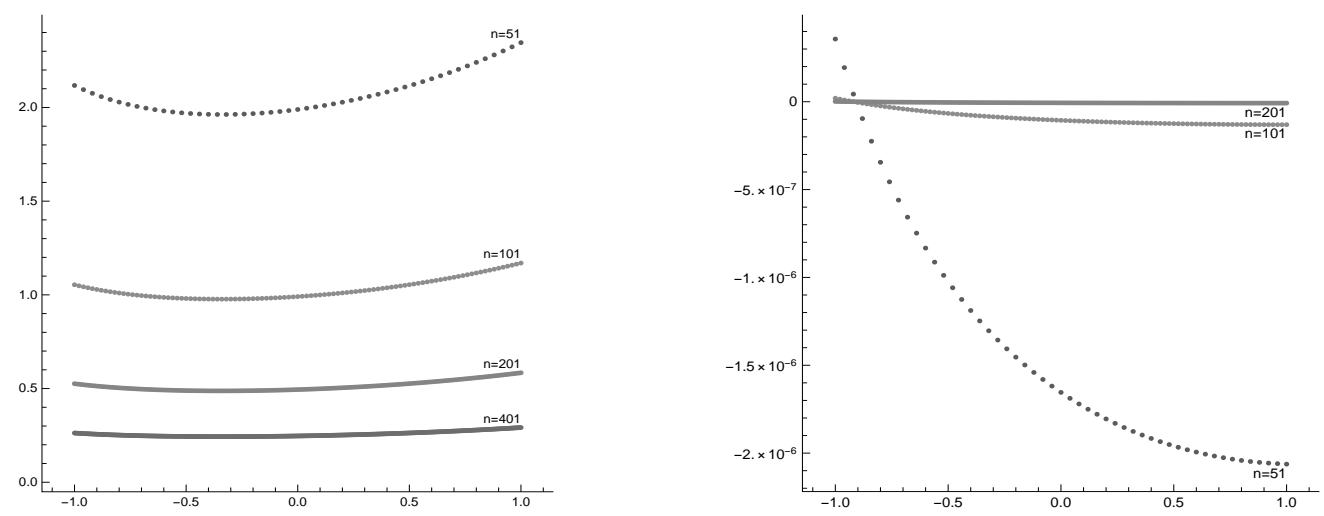

Abbildung 1.6: Relativer Fehler der Simulationslösung von FD1 (links) und FD2 (rechts) in Bezug zur Reihenlösung (1.11) auf $\mathfrak{Q}_{n, n}$ zum Zeitpunkt $t=4$ in Prozent bei Verwendung verschieden fein auflösender Rechengitter (Vertikalschnitt durch die Quadratmitte). 
Konvergenzverhalten der Simulation näher zu studieren und sich die von der Theorie vorhergesagte Konvergenzordnung bestätigen zu lassen, kann man — ausgehend vom Gitter $\mathfrak{Q}_{101,101}$ - die Abstände der Gitterpunkte sukzessive halbieren bzw. verdoppeln und sich wieder die daraus resultierenden relativen Fehler ausgeben lassen, vgl. Abbildung 1.6. Es bietet sich ferner an, den relativen Fehler der Lösung auf $\mathfrak{Q}_{101,101}$ als Referenz zu Grunde zu legen. Abbildung 1.7 zeigt den auf diese Art normierten relativen Fehler: Bei Änderung des Gitterabstandes um den Faktor zwei tritt für FD1 die lineare Fehlerordnung zu Tage, in logarithmischer Skalierung vermutet man bei $F D 2$ in etwa den Faktor 16, in Übereinstimmung mit der dem Verfahren eigenen Fehlerordnung $\mathcal{O}\left(\Delta^{4}\right)$. Diese Vermutung wird bestätigt, indem man sich den Quotienten zweier aufeinanderfolgender relativer Fehler grafisch ausgeben läßt.
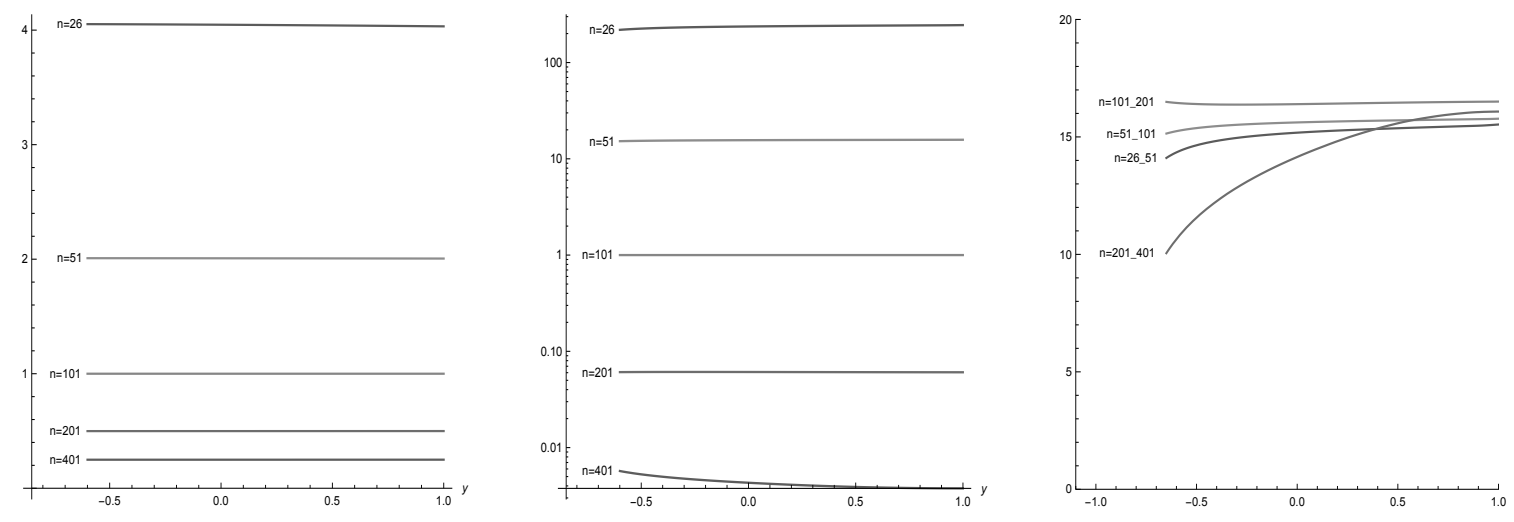

Abbildung 1.7: Mit der Lösung auf $\mathfrak{Q}_{101,101}$ normierter relativer Fehler der Simulationslösung von FD1 (links) und FD2 (Mitte) in Bezug zur Reihenlösung 1.11 zum Zeitpunkt $t=4$ in Prozent bei Verwendung verschieden fein aufösender Rechengitter (Vertikalschnitt durch die Quadratmitte); Konvergenzordnung von FD2 durch Quotientenbildung (rechts).

\begin{tabular}{|c||c|c|c|}
\hline \hline Verfahren & Anzahl Gitterpunkte & Absolutes Fehlermittel & errechnete Konvergenzordnung \\
\hline \hline$F D 1$ & 2.601 & 0.00627 & - \\
\hline$F D 1$ & 10.201 & 0.00312 & 2.00717 \\
\hline$F D 1$ & 40.401 & 0.00156 & 2.00360 \\
\hline$F D 2$ & 2.601 & $4.67382 * 10^{-9}$ & - \\
\hline$F D 2$ & 10.201 & $2.99177 * 10^{-10}$ & 15.62228 \\
\hline$F D 2$ & 40.401 & $1.82074 * 10^{-11}$ & - \\
\hline$P d e T b x$ & 2.577 & $3.47640 * 10^{-6}$ & 4.02199 \\
\hline$P d e T b x$ & 10.145 & $8.64349 * 10^{-7}$ & 4.00349 \\
\hline$P d e T b x$ & 40.257 & $2.15899 * 10^{-7}$ & - \\
\hline$F D 2 \sqrt[4.6]{F D 2} \overline{4.6}$ & 2.601 & $4.67351 * 10^{-9}$ & 15.67369 \\
\hline$F D 2 \sqrt{4.6}$ & 10.201 & $2.98176 * 10^{-10}$ & 16.02313 \\
\hline
\end{tabular}

Tabelle 1.1: Vergleich der arithmetischen Mittel des absoluten Fehlers für $t=4$.

Betrachtet man zur Bewertung der Verfahren im jeweiligen Gitterpunkt den absoluten Fehler $\left|u\left(x_{i}, y_{j}, t\right)-\hat{u}\left(x_{i}, y_{j}, t\right)\right|$, summiert diesen über alle Gitterpunkte und dividiert anschließend durch deren Anzahl, so liefert dies ein Vergleichskriterium. Diese Vorgehensweise scheint auch sinnvoll, da die PDE-Toolbox auf Grund der Verwendung eines unstrukturierten Rechengitters zu einer anderen Anzahl an Rechengitterpunkten gelangt. Abbildung 1.8 visualisiert die absoluten Fehler am Rechengitter bei zunehmender Gitterverfeinerung, Tabelle 1.1 gibt eine Übersicht über die arithmetischen Fehlermittel und die Konvergenzordnungen, für die PDE-Toolbox wurde dabei eine quadratische Fehlerordnung ermittelt. Am Tabellenende sind auch schon die Simulationsresultate aus Abschnitt 4.6 bei konformer Abbildung des Quadrates über den Einheitskreis auf sich selbst angeführt. 


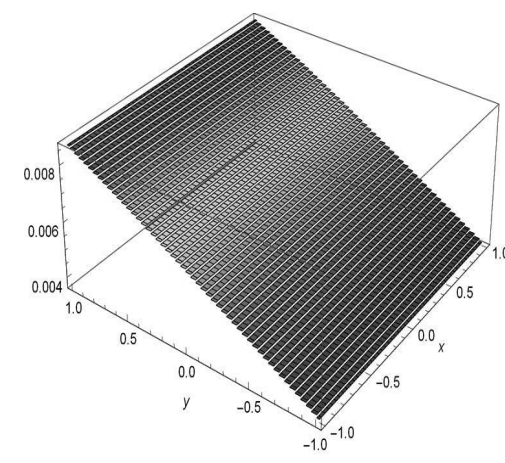

FD1 2.601 Pt

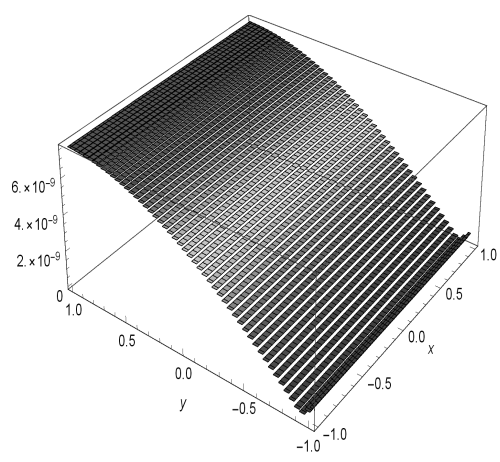

FD2 2.601 Pt

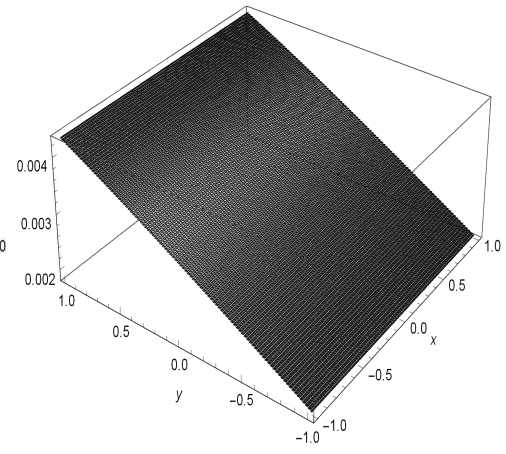

FD1 10.201 Pt

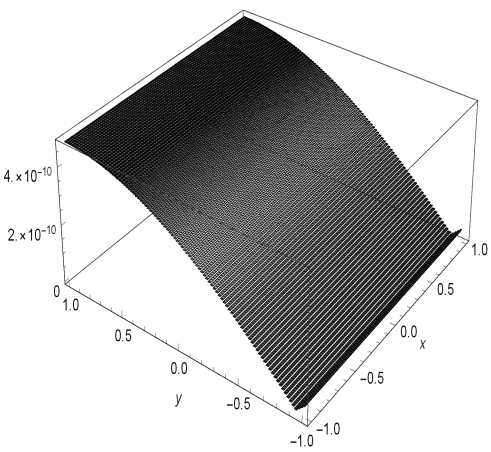

FD2 10.201 Pt

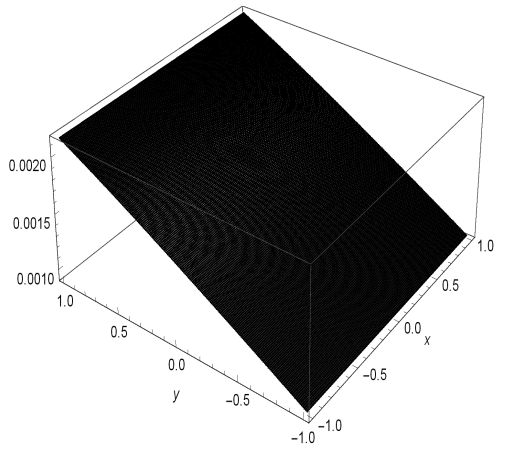

FD1 $40.401 \mathrm{Pt}$

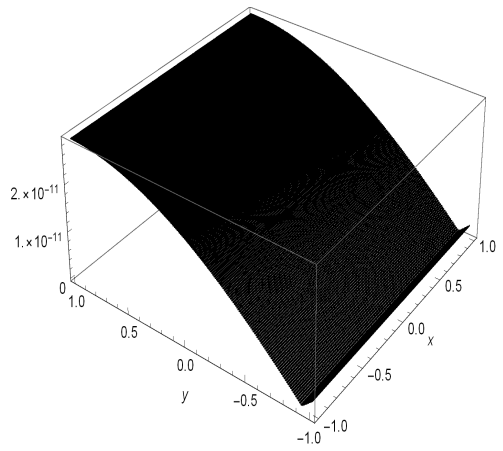

FD2 40.401 Pt

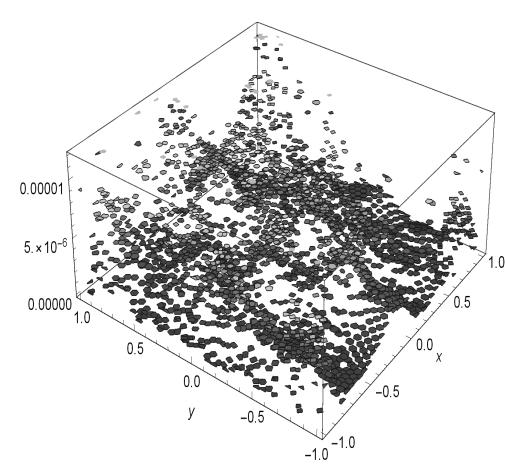

PdeTbx $2.577 \mathrm{Pt}$

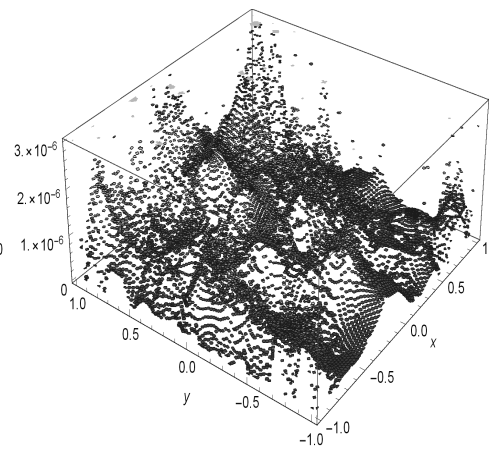

PdeTbx 10.145 Pt

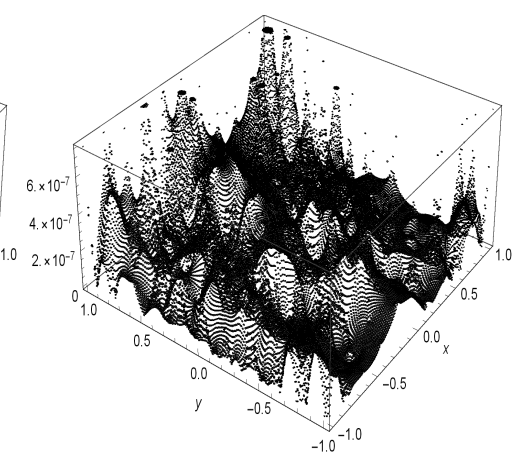

PdeTbx $40.257 \mathrm{Pt}$

Abbildung 1.8: Vergleich der absoluten Fehler bei sukzessiver Gitterverfeinerung. 


\subsection{Finite Differenzen}

Die bislang dargelegte Methodik der Diskretisierung der in einer partiellen Differentialgleichung auftretenden Ortsableitungen zur Überführung in ein System gewöhnlicher Differentialgleichungen zeigt auf, daß es wünschenswert wäre, formelmäßige Näherungsausdrücke für beliebige Differentialquotienten an der Hand zu haben. Ziel dieses Abschnittes ist daher die Approximation von (gegebenenfalls gemischten) partiellen Ableitungen beliebiger Ordnung einer reellwertigen Funktion $u(x, y)$ in zwei unabhängigen reellen Veränderlichen auf einem regulären Rechengitter. Der Abstand der Gitterpunkte sei dazu gemäß der Definition des Rechengitters $\mathfrak{Q}_{n_{1}, n_{2}}$ in die jeweilige Koordinatenrichtung mit $\Delta_{x}$ und $\Delta_{y}$ äquidistant gewählt. Die Vorgehensweise in diesem Abschnitt orientiert sich an den Ausführungen von ForNBERG ([12], [13], [14]).

Ausgehend von der zweidimensionalen Fourier-Entwicklung der Funktion $u(x, y)$, deren Periodizitätsbereich so eingerichtet werde, daß sich das diskrete Rechengitter im Inneren befindet,

$$
u(x, y) \sim \sum_{h, k=-\infty}^{\infty} c_{h k} e^{i\left(\omega_{h} x+\omega_{k} y\right)}
$$

wählt man zur Approximation der partiellen Ableitung im Punkt $(x, y)$ den Ansatz

$$
\frac{\partial^{\mu+\nu} u(x, y)}{\partial^{\mu} x \partial^{\nu} y} \approx \sum_{m=-m_{0}}^{m_{1}-m_{0}} \sum_{n=-n_{0}}^{n_{1}-n_{0}} a_{m n} u\left(x+m \Delta_{x}, y+n \Delta_{y}\right) .
$$

Dabei bedeuten

- $a_{m n} \ldots$ die gesuchten Gewichte der Approximation

- $\mu, \nu \ldots$ Ordnung der Ableitung in $x$ - und $y$-Richtung

- $m_{1}, n_{1} \ldots$ Anzahl der Intervalle zwischen den jeweils äußersten Gitterpunkten

- $m_{0}, n_{0} \ldots$ Anzahl der Intervalle zwischen Entwicklungspunkt und linkem (unterem) Punkt

Setzt man in diesen Ansatz die Fourier-Reihe der Funktion $u$ ein, so folgt durch Ausdifferenzieren und Umsortieren

$$
\begin{aligned}
\frac{\partial^{\mu+\nu} u(x, y)}{\partial^{\mu} x \partial^{\nu} y} & \sim \sum_{h, k=-\infty}^{\infty}\left[i^{\mu+\nu} \omega_{h}^{\mu} \omega_{k}^{\nu}\right] c_{h k} e^{i\left(\omega_{h} x+\omega_{k} y\right)} \\
& \approx \sum_{m=-m_{0}}^{m_{1}-m_{0}} \sum_{n=-n_{0}}^{n_{1}-n_{0}} a_{m n} \sum_{h, k=-\infty}^{\infty} c_{h k} e^{i\left(\omega_{h} x+\omega_{k} y\right)} e^{i\left(\omega_{h} m \Delta_{x}+\omega_{k} n \Delta_{y}\right)} \\
& =\sum_{h, k=-\infty}^{\infty}\left[\sum_{m=-m_{0}}^{m_{1}-m_{0}} \sum_{n=-n_{0}}^{n_{1}-n_{0}} a_{m n} e^{i\left(\omega_{h} m \Delta_{x}+\omega_{k} n \Delta_{y}\right)}\right] c_{h k} e^{i\left(\omega_{h} x+\omega_{k} y\right)},
\end{aligned}
$$

und man erhält durch Koeffizientenvergleich $\forall h, k \in Z$ den Zusammenhang

$$
i^{\mu+\nu} \omega_{h}^{\mu} \omega_{k}^{\nu} \approx \sum_{m=-m_{0}}^{m_{1}-m_{0}} \sum_{n=-n_{0}}^{n_{1}-n_{0}} a_{m n} e^{i\left(\omega_{h} m \Delta_{x}+\omega_{k} n \Delta_{y}\right)} .
$$

Mit den Substitutionen $\xi=e^{i \omega_{h} \Delta_{x}}$ bzw. $\eta=e^{i \omega_{k} \Delta_{y}}$ folgt mit $\omega_{h}=\ln \xi / i \Delta_{x}$ bzw. $\omega_{k}=\ln \eta / i \Delta_{y}$

$$
i^{\mu+\nu}\left(\frac{\ln \xi}{i \Delta_{x}}\right)^{\mu}\left(\frac{\ln \eta}{i \Delta_{y}}\right)^{\nu} \approx \sum_{m=-m_{0}}^{m_{1}-m_{0}} \sum_{n=-n_{0}}^{n_{1}-n_{0}} a_{m n} \xi^{m} \eta^{n}
$$


Für die linke Seite findet man jetzt bequem das Taylor-Polynom in $\xi$ und $\eta$ im Entwicklungspunkt $(\xi=1, \eta=1)$ - es ist ja die ursprüngliche Funktion um $\Delta_{x}=\Delta_{y}=0$ zu entwickeln — und nach Umformung ergibt sich ein Zusammenhang, aus dem durch Koeffizientenvergleich der aus der Taylor-Entwicklung bekannten $b_{m n}$ die gesuchten Gewichte $a_{m n}$ der FD-Approximation ermittelt werden können,

$$
\frac{\xi^{m_{0}} \eta^{n_{0}}(\ln \xi)^{\mu}(\ln \eta)^{\nu}}{\Delta_{x}{ }^{\mu} \Delta_{y}^{\nu}} \approx \sum_{m=0}^{m_{1}} \sum_{n=0}^{n_{1}} b_{m n}(\xi-1)^{m}(\eta-1)^{n}=\sum_{m=0}^{m_{1}} \sum_{n=0}^{n_{1}} a_{m-m_{0}, n-n_{0}} \xi^{m} \eta^{n} .
$$

Zur besseren Veranschaulichung der jeweiligen Formel können die Gewichte in eine Matrix zusammengefaßst werden, wobei das Gewicht des Entwicklungspunktes $a_{00}$ speziell gekennzeichnet wird. Die Approximation der Ableitung erhält man dann durch Aufsummieren sämtlicher Produkte $a_{m n} u_{m n}$ für $m=-m_{0} \ldots m_{1}$ und $n=-n_{0} \ldots n_{1}$. Nachfolgend soll die konkrete Vorgehensweise an Hand einiger Beispiele demonstriert werden, die gefundenen Ausdrücke wurden speziell auch zur Implementierung der Approximationen der Ortsableitungen herangezogen.

Beispiel 1. 2-Punkt-Molekül, erste Ableitung. Daß Formel 1.22 auch im eindimensionalen Fall anwendbar ist, ersieht man etwa an Hand der Vorwärtsdifferenz für die Approximation von $u^{\prime}(x)$. In diesem Fall ist $\mu=1, \nu=0, m_{1}=1$ und $m_{0}=n_{0}=n_{1}=0$, man entwickelt demnach um $\xi=1$ in die Reihe

$$
\frac{\ln \xi}{\Delta_{x}} \approx \frac{\xi-1}{\Delta_{x}}
$$

das Molekül der FD-Gewichte lautet also gemäß $u^{\prime}(0) \approx\left(u_{1}-u_{0}\right) / \Delta_{x}$

$$
\frac{1}{\Delta_{x}}\left(\begin{array}{ll}
-1 & 1
\end{array}\right)
$$

Beispiel 2. 5-Punkt-Moleküle, erste Ableitung. Zur Diskretisierung der ersten Ableitung für einen Randpunkt des Rechengitters in $x$-Richtung, wie sie etwa bei der Umsetzung von NEUMANNschen oder NEWTONschen Randbedingungen gesucht ist, wird für fünf Gitterpunkte am linken Gitterrand $\mu=1, \nu=0, m_{1}=4$ und $m_{0}=n_{0}=n_{1}=0$. Man findet mit

$$
\frac{\ln \xi}{\Delta_{x}} \approx \frac{\xi-1}{\Delta_{x}}-\frac{(\xi-1)^{2}}{2 \Delta_{x}}+\frac{(\xi-1)^{3}}{3 \Delta_{x}}-\frac{(\xi-1)^{4}}{4 \Delta_{x}}=\frac{1}{12 \Delta_{x}}\left(-25+48 \xi-36 \xi^{2}+16 \xi^{3}-3 \xi^{4}\right)
$$

die Approximation $u^{\prime}(0) \approx\left(-3 u_{4}+16 u_{3}-36 u_{2}+48 u_{1}-25 u_{0}\right) / 12 \Delta_{x}$ und damit das RandpunktMolekül

$$
\frac{1}{12 \Delta_{x}}\left(\begin{array}{lllll}
-25 & 48 & -36 & 16 & -3
\end{array}\right)
$$

Weiters erhält man für $m_{0}=1, \ldots, 4$ die Moleküle

$$
\begin{aligned}
& \frac{1}{12 \Delta_{x}}\left(\begin{array}{llllll}
-3 & -10 & 18 & -6 & 1
\end{array}\right) \\
& \frac{1}{12 \Delta_{x}}\left(\begin{array}{llllll}
1 & -8 & 0 & 8 & -1
\end{array}\right) \\
& \frac{1}{12 \Delta_{x}}\left(\begin{array}{llllll}
-1 & 6 & -18 & 10 & 3
\end{array}\right) \\
& \frac{1}{12 \Delta_{x}}\left(\begin{array}{lllll}
3 & -16 & 36 & -48 & 25
\end{array}\right),
\end{aligned}
$$

in allen Fällen dieses Beispiels ist die Approximationsgüte mindestens von vierter Ordnung. 
Beispiel 3. 6-Punkt-Moleküle, zweite Ableitung. Um zu einer Approximationsgenauigkeit von mindestens vierter Ordnung für die zweite Ableitung in $y$-Richtung zu gelangen, sind, wie eine Betrachtung der Taylorreihe zeigt, im allgemeinen sechs Gitterpunkte notwendig. Mit $\mu=0$, $\nu=2, n_{1}=5$ und $m_{0}=m_{1}=0$ sind die gesuchten Entwicklungen für $n_{0}=0, \ldots 5$ nach 1.22

$$
\begin{aligned}
\frac{\eta^{n_{0}}(\ln \eta)^{2}}{\Delta_{y}^{2}} \approx \frac{1}{12 \Delta_{y}^{2}} & {\left[45-51 n_{0}+18 n_{0}^{2}-2 n_{0}^{3}+\left(-154+213 n_{0}-84 n_{0}^{2}+10 n_{0}^{3}\right) \eta+\right.} \\
& \left(214-354 n_{0}+156 n_{0}^{2}-20 n_{0}^{3}\right) \eta^{2}+\left(-156+294 n_{0}-144 n_{0}^{2}+20 n_{0}^{3}\right) \eta^{3}+ \\
& \left.\left(61-123 n_{0}+66 n_{0}^{2}-10 n_{0}^{3}\right) \eta^{4}+\left(-10+21 n_{0}-12 n_{0}^{2}+2 n_{0}^{3}\right) \eta^{5}\right] .
\end{aligned}
$$

Dies erbringt für $n_{0}=0, \ldots 5$ sukzessive die folgenden Moleküle (gemeinsamer Vorfaktor $1 / 12 \Delta_{y}^{2}$ ):

$$
\left.\left(\begin{array}{r}
-10 \\
61 \\
-156 \\
214 \\
-154 \\
45
\end{array}\right) \quad\left(\begin{array}{r}
1 \\
-6 \\
14 \\
-4 \\
-15 \\
10
\end{array}\right) \quad\left(\begin{array}{r}
0 \\
-1 \\
16 \\
-30 \\
16 \\
-1
\end{array}\right) \quad\left(\begin{array}{r}
-1 \\
16 \\
-30 \\
16 \\
-1 \\
0
\end{array}\right) \quad\left(\begin{array}{r}
10 \\
-15 \\
-4 \\
14 \\
-6 \\
1
\end{array}\right) \quad \begin{array}{r}
45 \\
-154 \\
214 \\
-156 \\
61 \\
-10
\end{array}\right)
$$

Bemerkenswert ist neben der Symmetrie auch noch der Umstand, daß für die zur Gänze im Inneren des Gitters gelegenen Punkte jeweils ein FD-Gewicht verschwindet. Somit sind zur Approximation der zweiten Ableitung mit einer Genauigkeit von vierter Ordnung nur fünf Punkte notwendig, und zwar der Punkt selbst nebst seinen vier Nachbarpunkten. Es ist dies die bereits angeführte Formel

$$
u_{x x}=\frac{-u_{-2}+16 u_{-1}-30 u_{0}+16 u_{1}-u_{2}}{12 h^{2}}+\frac{\mathcal{O}\left(h^{6}\right)}{h^{2}} .
$$

Beispiel 4. 25-Punkt-Moleküle, gemischte partielle Ableitung. Der Fehler möge dabei wieder von vierter Ordnung gemäß $\mathcal{O}\left(\Delta_{x}^{4}\right)+\mathcal{O}\left(\Delta_{y}^{4}\right)$ gegen Null streben, dazu ist ein Molekül mit 25 Punkten erforderlich. Je nach Lage des Entwicklungspunktes sind hier diverse Fälle zu unterscheiden. Der Fall $m_{0}=n_{0}=2, m_{1}=n_{1}=4$ kann nur dann angewendet werden, wenn der entsprechende Gitterpunkt hinreichend im Inneren des Rechengebietes gelegen ist in dem Sinne, daß die Funktionswerte $u_{2-m, 2-n}$ für $m, n=0, \ldots 4$ zur Verfügung stehen. Es ist für $\partial^{2} f / \partial x \partial y$ in Formel 1.22 $\mu=\nu=1 \mathrm{zu}$ setzen, somit folgt ${ }^{5}$ aus

$$
\begin{array}{r}
\frac{\xi^{2} \eta^{2} \ln \xi \ln \eta}{\Delta_{x} \Delta_{y}} \approx \frac{1}{144 \Delta_{x} \Delta_{y}}\left[\begin{array}{r}
1-8 \xi+8 \xi^{3}-\xi^{4}-8 \eta+64 \xi \eta-64 \xi^{3} \eta+8 \xi^{4} \eta+ \\
8 \eta^{3}-64 \xi \eta^{3}+64 \xi^{3} \eta^{3}-\eta^{4}+8 \xi \eta^{4}-8 \xi^{3} \eta^{4}+\xi^{4} \eta^{4}
\end{array}\right]
\end{array}
$$

das Zentral-Molekül, in dem neun Gewichte verschwinden:

$$
\frac{1}{144 \Delta_{x} \Delta_{y}}\left(\begin{array}{ccccc}
-1 & 8 & 0 & -8 & 1 \\
8 & -64 & 0 & 64 & -8 \\
0 & 0 & 0 & 0 & 0 \\
-8 & 64 & 0 & -64 & 8 \\
1 & -8 & 0 & 8 & -1
\end{array}\right)
$$

\footnotetext{
${ }^{5}$ Alle diese TAYLOR-Entwicklungen läßt man natürlich von Mathematica durchführen, hier:

MatrixForm [CoefficientList [Normal [Series $\left.\left.\left[\mathrm{x}^{2} * \mathrm{y}^{2} * \log [\mathrm{x}] * \log [\mathrm{y}],\{\mathrm{x}, 1,4\},\{\mathrm{y}, 1,4\}\right] /(\mathrm{h} * \mathrm{k})\right],\{\mathrm{x}, \mathrm{y}\}\right]$ ]
} 
Weitere, nicht-zentrale Moleküle für dieses Beispiel lauten (Vorfaktor jeweils $1 / 144 \Delta_{x} \Delta_{y}$ ) für den Fall der vier Punkte in der Nähe der Quadratecken

$$
\begin{gathered}
\left(\begin{array}{ccccc}
-3 & -10 & 18 & -6 & 1 \\
18 & 60 & -108 & 36 & -6 \\
-54 & -180 & 324 & -108 & 18 \\
30 & 100 & -180 & 60 & -10 \\
9 & 30 & -54 & 18 & -3
\end{array}\right)\left(\begin{array}{cccccc}
-9 & -30 & 54 & -18 & 3 \\
-30 & -100 & 180 & -60 & 10 \\
54 & 180 & -324 & 108 & -18 \\
-18 & -60 & 108 & -36 & 6 \\
3 & 10 & -18 & 6 & -1
\end{array}\right) \\
\left(\begin{array}{ccccc}
-1 & 6 & -18 & 10 & 3 \\
6 & -36 & 108 & -60 & -18 \\
-18 & 108 & -324 & 180 & 54 \\
10 & -60 & 180 & -100 & -30 \\
3 & -18 & 54 & -30 & -9
\end{array}\right)\left(\begin{array}{ccccc}
-3 & 18 & -54 & 30 & 9 \\
-10 & 60 & -180 & 100 & 30 \\
18 & -108 & 324 & -180 & -54 \\
-6 & 36 & -108 & 60 & 18 \\
1 & -6 & 18 & -10 & -3
\end{array}\right),
\end{gathered}
$$

wobei man erkennt, daß die Spiegelung an einer der Achsen einen Vorzeichenwechsel bewirkt. Die resultierenden Matrizen sind außerdem vollbesetzt.

Für Entwicklungspunkte in Randnähe, denen nur in eine Koordinatenrichtung Funktionswerte unvollständig zur Verfügung gestellt werden können, findet man

$$
\begin{gathered}
\left(\begin{array}{ccccc}
3 & 10 & -18 & 6 & -1 \\
-24 & -80 & 144 & -48 & 8 \\
0 & 0 & 0 & 0 & 0 \\
24 & 80 & -144 & 48 & -8 \\
-3 & -10 & 18 & -6 & 1
\end{array}\right)\left(\begin{array}{ccccc}
1 & -8 & 0 & 8 & -1 \\
-6 & 48 & 0 & -48 & 6 \\
18 & -144 & 0 & 144 & -18 \\
-10 & 80 & 0 & -80 & 10 \\
-3 & 24 & 0 & -24 & 3
\end{array}\right) \\
\left(\begin{array}{ccccc}
3 & -24 & 0 & 24 & -3 \\
10 & -80 & 0 & 80 & -10 \\
-18 & 144 & 0 & -144 & 18 \\
6 & -48 & 0 & 48 & -6 \\
-1 & 8 & 0 & -8 & 1
\end{array}\right)\left(\begin{array}{ccccc}
1 & -6 & 18 & -10 & -3 \\
-8 & 48 & -144 & 80 & 24 \\
0 & 0 & 0 & 0 & 0 \\
8 & -48 & 144 & -80 & -24 \\
-1 & 6 & -18 & 10 & 3
\end{array}\right)
\end{gathered}
$$

wiederum wird durch Spiegelung ein Vorzeichenwechsel bewirkt, zudem verschwinden jeweils fünf Gewichte. Für Moleküle ,echter“ Randpunkte verfährt man analog, falls eine Diskretisierung der gemischten partiellen Ableitung bei Vorliegen entsprechender Randbedingungen notwendig ist.

Bei den in Beispiel 4 angegebenen Formeln darf aber nicht übersehen werden, daß erstmals ein Fall vorliegt, bei dem auch Werte in den Quadrateckpunkten zur Verfügung gestellt werden müssen. Dieser zunächst harmlos anmutende Sachverhalt wirft bei näherer Betrachtung Fragestellungen prinzipieller Natur auf, nämlich wie zur Ermittlung dieser vier Funktionswerte vorgegangen werden kann, wenn an der Berandung keine Bedingungen vom DiRICHLETschen Typ vorgeschrieben sind. Einen Ansatz stellt die Forderung nach entsprechenden Stetigkeits- oder Differenzierbarkeitseigenschaften in diesen Eckpunkten dar, wie sie im übrigen auch bei Kollision von strukturell verschiedenen Randbedingungen in Punkten auf den Quadratseiten zu fordern sind. Alternativ dazu kann auch versucht werden, diese Problempunkte durch Verwendung geeigneterer Moleküle zu umschiffen.

Mit den Ergebnissen dieses Abschnittes ist man also zusätzlich in der Lage, die Fehlerordnung eines Verfahrens unter Auswahl zweckmäßiger Diskretisierungsmethoden für die auftretenden Differentialquotienten zu steuern. Es gilt, den Entwicklungsaufwand und die Fehleranfälligkeit bei Umsetzung der Implementierung (die Näherungsausdrücke können dabei unter Minimierung des Entwicklungsaufwandes von einer externen Datei eingelesen werden) gegen die resultierende Konvergenzordnung abzuwägen. Da in den Fällen von praktischer Relevanz eine Referenzlösung im allgemeinen nicht zugänglich sein wird, kann man im Zuge einer Simulationsstudie Verfahren von unterschiedlicher Fehlerordnung einsetzen und die Ergebnisse dann unter Verfeinerung des Rechengitters im Hinblick auf Konvergenz und Konsistenz prüfen. 


\subsection{Fazit und Argumente für die konforme Abbildung}

Insgesamt kann resümiert werden, daß die Vorteile der Linienmethode gegenüber der Methode der Finiten Elemente, wie sie am Beispiel der PDE-Toolbox studiert wurde, hinsichtlich Steuerung der Konvergenzordnung und der sich daraus ergebenden Fehlergenauigkeit in diesem einleitenden Kapitel sehr deutlich zu Tage getreten sind. Ein gravierender Nachteil besteht allerdings in dem Umstand, daß der Prozeß der Ortsdiskretisierung gebunden ist an ein reguläres, typischerweise äquidistantes Rechengitter, obwohl er sich andererseits recht einfach auch auf höherdimensionale Modelle mit verteilten Parametern erweitern läßt. Diese Einschränkung gilt für FE-Methoden nicht, durch eine Rechengittergenerierung mittels Triangulierung ist die in Betracht stehende Geometrie praktisch keinen Beschränkungen unterworfen. Der höheren Flexibilität hinsichtlich der Gestalt des Rechengebietes steht bei der PDE-Toolbox jedoch eine stets quadratische Fehlerordnung gegenüber.

Ein weiterer Aspekt betrifft die mögliche Breite der Anwendbarkeit hinsichtlich der verschiedenen Klassen partieller Differentialgleichungen. Die vorgestellte Implementierung der Linienmethode eignet sich primär für instationäre Problemstellungen (stationäre Aufgabenstellung sind aber prinzipiell auch abgedeckt) und ist dahingehend verwendbar für beliebige Systeme partieller Differentialgleichungen. In der PDE-Toolbox besteht aus der Blackbox lediglich die Möglichkeit der Auswahl aus Gleichungen von sehr speziellem Typ unter Angabe von Parametern. So ist zum Beispiel das Einbringen eines dynamischen Quellterms in die Wärmeleitungsgleichung aus der GUI nicht möglich, dazu hat man dann die tiefer liegenden Routinen in der Commandline zu verwenden.

Des weiteren ist zu hinterfragen, inwiefern die bei den Vergleichen verwendete PDE-Toolbox den zeitgemäßen Einsatz der Methode der Finiten Elemente repräsentieren kann. Auch wenn seitens Mathworks eine aktive Versionspflege stattfindet (mit der aktuellen Release von Matlab $2019 b$ wurde die PDE-Toolbox 3.3 ausgerollt), sei an dieser Stelle erwähnt, daß durch die Abspaltung und eigenständige Weiterentwicklung über FEMLAB zu COMSOL die Möglichkeit bestehen könnte, durch Einsatz dieser Software zu besseren Simulationsergebnissen hinsichtlich FE-Methoden zu gelangen.

Läßst unser bisher entwickeltes Mathematica-Programm zwar grundsätzlich auch schon die Anwendung auf instationäre Problemstellungen abseits der Wärmeleitungsgleichung zu, so ist es im Hinblick auf die Gestalt der zu Grunde liegenden Geometrie in Form des Einheitsquadrates sehr starken Einschränkungen unterworfen. Damit entsteht die Fragestellung, ob die für die Simulation hervorragend geeigneten Eigenschaften der CTDS-Linienmethode nutzbringend auf allgemeinere Gebiete unter Beibehaltung der Regularität des Rechengitters erweiterbar sind.

Die Antwort darauf gibt die konforme Abbildung des Einheitsquadrates: Durch ihre Eigenschaft der Winkeltreue werden lokale Quadratnetze im Kleinen wieder auf Quadratnetze abgebildet, der Quadratrand kommt ferner mit dem Rand der in Betracht stehenden Geometrie zur Deckung und schließlich weist auf der Berandung der Geometrie das Bild jeweils eines der kartesischen Basisvektoren in Richtung des dortigen Normalenvektors (die Orthogonalität der Basisvektoren bleibt somit erhalten, nicht aber deren Länge und auch nicht deren Konstanz). Die Herausforderung besteht demnach für ein vorgegebenes Gebiet zunächst in der numerischen Konstruktion einer solchen konformen Transformation, um diese dann nutzbringend für die Simulation einzusetzen. 


\section{Kapitel 2}

\section{Theorie der konformen Abbildung}

Die konstruktive Theorie der konformen Abbildung hat in ihrer Eigenschaft als Teildisziplin der komplexen Analysis alte Wurzeln, bereits Anfang des 20. Jahrhunderts suchte man etwa in Ermangelung leistungsfähiger Rechenanlagen nach Methoden, die eine effiziente Berechnung von Tragflügelprofilen (N. JoukOwski, 1906) ermöglichen sollten. Es zeigte sich, daß durch die Anwendung konformer Transformationen die Verkomplizierung der Gestalt von Differentialoperatoren — bei gleichzeitiger Vereinfachung des Rechengebietes - auf ein Mindestmaß reduziert werden konnte und damit für die damaligen Verhältnisse notwendigen Handrechnungen essentielle Vereinfachungen erbrachte.

Es ist wenig verwunderlich, daß in der heutigen Zeit, in der den Taktfrequenzen von Microprozessoren eine weniger bedeutende Rolle als noch vor 30 Jahren zukommt und zudem Rechenleistung aus der Cloud nach Bedarf erworben werden kann, moderne Simulatoren dem Bestreben nach Vereinfachung der Dinge weniger Augenmerk entgegenbringen.

Auch scheint dadurch der Trend in der Simulation partieller Differentialgleichungen in die Richtung unstrukturierter Rechennetze zu weisen, und damit gerät die Methode der Finiten Differenzen, deren Stärken ja auf orthogonalen Gittern zu finden sind, zunehmend ins Abseits - und das mitunter ganz zu Recht, denn strukturierte Gitter verlangen - beispielsweise wenn Randbedingungen in Richtung des auswärts weisenden Normalenvektors vorgeschrieben werden - in den meisten Fällen nach einer regelmäßigen Berandung und führen damit zu Aufgabenstellungen, die man mithin als von akademischer Natur bezeichnen kann.

Nun kann man sich aber durchaus die Frage stellen, ob nicht eine Möglichkeit besteht, die Vorzüge der Finiten Differenzen auch auf unregelmäßig berandeten Rechengebieten auszuspielen. Genau das wird durch Zwischenschaltung der konformen Abbildung, deren Stärke die winkeltreue Überführung von Rechennetzen unter Anpassung auf die Geometrie der Berandung ist, geleistet.

Das vorliegende Kapitel widmet sich daher der Aufgabenstellung, das in Kapitel 1 verwendete Rechengitter $\mathfrak{Q}_{n_{1}, n_{2}}$ aus Abbildung 1.4 dergestalt auf ein vorgegebenes Gebiet zu deformieren, daß einerseits die Orthogonalität in den Rechenpunkten des Inneren erhalten bleibt und andererseits eine umkehrbar eindeutige Abbildung der Berandung erzielt wird. Während dabei manche Abbildungen, wie etwa die Transformation des Einheitsquadrates auf den Einheitskreis oder die des Kreises auf den Kreiszwickel, durch geschlossene Ausdrücke angegeben werden können, bedarf es zur Konstruktion von Abbildungen auf allgemeinere Geometrien der Lösung von Integralgleichungen (linearer oder nichtlinearer Natur), die den Zusammenhang am Rand (Ränderzuordnungsfunktion) herstellen.

Es liegt in der Natur der zu Grunde liegenden komplexen Analysis, daß die Betrachtungen auf zweidimensionale, ebene Abbildungsprobleme beschränkt bleiben. 


\subsection{Elemente der Funktionentheorie}

Für das Folgende bezeichne $\mathbb{C}$ den Körper der komplexen Zahlen mit den vertrauten Eigenschaften und Rechenregeln. Zur Klärung der Begriffsbildungen folgen zunächst einige Definitionen.

Für eine nicht-leere Teilmenge $M \subseteq \mathbb{C}$ und einen Punkt $\zeta_{0}=\xi_{0}+i \eta_{0} \in \mathbb{C}$ sei mit [30, S.7f.]

$$
K_{\varepsilon}\left(\zeta_{0}\right):=\left\{\zeta \in \mathbb{C}|| \zeta-\zeta_{0} \mid<\varepsilon\right\}
$$

die $\varepsilon$-Umgebung von $\zeta_{0}$ in Form einer offenen Kreisscheibe mit Radius $\varepsilon>0$ eingeführt. $\zeta_{0}$ heißt

- Innerer Punkt von $M$, wenn $\exists \varepsilon: K_{\varepsilon}\left(\zeta_{0}\right) \subseteq M$

- Äußerer Punkt von $M$, wenn $\exists \varepsilon: K_{\varepsilon}\left(\zeta_{0}\right) \subseteq \mathbb{C} \backslash M$

- Randpunkt von $M$, wenn $\zeta_{0}$ weder innerer noch äußerer Punkt von $M$ ist.

$\zeta_{0}$ ist dabei exklusiv entweder innerer Punkt, äußerer Punkt oder Randpunkt von $M$. Besteht $M$ nur aus inneren Punkten, so heißt $M$ offen.

$M$ wird wegzusammenhängend genannt ${ }^{1}$, wenn sich je zwei beliebige Punkte $\zeta=\zeta_{0}, \hat{\zeta}=\zeta_{n} \in M$ durch einen ganz in $M$ verlaufenden Polygonzug verbinden lassen,

$$
\bigcup_{i=1}^{n}\left\{(1-t) \zeta_{i-1}+t \zeta_{i} \mid t \in[0,1]\right\} \subseteq M .
$$

Ein Gebiet $\mathfrak{G}$ ist definiert als nicht-leere, offene und zusammenhängende Teilmenge von $\mathbb{C}$.

Lassen sich auf einer zusammenhängenden Menge $M$ für irgend zwei beliebige Punkte je zwei solcher die beiden Punkte verbindende Polygonzüge innerhalb von $M$ stetig ineinander überführen, so heißt $M$ einfach zusammenhängend.

Ist $[a, b]$ ein Intervall, so nennt man eine Abbildung $\gamma:[a, b] \rightarrow \mathfrak{C} \subset \mathbb{C}$ eine Kurve. Ist $\gamma(t)$ injektiv $^{2}$ und stetig, spricht man von einem Jordan-Bogen, gilt zusätzlich $\gamma(a)=\gamma(b)$ von einer (geschlossenen) Jordan-Kurve. Ist $\gamma(t) \forall t \in[a, b]$ nach dem Kurvenparameter stetig differenzierbar mit $\dot{\gamma}(t) \neq 0$, dann heißt $\gamma$ glatt, im Falle beliebig oftmaliger stetiger Differenzierbarkeit analytisch. Damit ist klar, was unter einer stückweise glatten (bzw. stückweise analytischen) Jordan-Kurve zu verstehen ist: Hier liegen endlich viele Ecken vor, in denen die links- und rechtsseitigen Ableitungen zwar existieren, jedoch nicht übereinstimmen.

Durch die Punktmenge $\mathfrak{C}$ einer stückweise glatten Jordan-Kurve $\gamma$ wird $\mathbb{C}$ in zwei disjunkte, einfach zusammenhängende Gebiete $\mathfrak{G}_{1}$ und $\mathfrak{G}_{2}$ geteilt ${ }^{3}$, wobei bei positiver Orientierung von $\gamma$ die zur Linken liegende Punktmenge $\mathfrak{G}_{1}$ das Innere von $\mathfrak{C}$ genannt wird. $\mathfrak{G}_{1}$ wird also von $\mathfrak{C}$ berandet.

Ist eine komplexe Funktion $\zeta=f(z)$ auf einem Gebiet $\mathfrak{G} \subseteq \mathbb{C}$ definiert und dort überall differenzierbar, so heißt $f$ analytisch ${ }^{4}$. Nach dem Satz über die Gebietstreue [7, S.1283] ist das Bild $f(\mathfrak{G})$ einer nicht-konstanten holomorphen Funktion wieder ein Gebiet, die Forderung $f^{\prime}(z) \neq 0$ ist jedoch bemerkenswerter Weise keine hinreichende Bedingung für die Bijektivität von $f$.

Laut dem Ersten Fundamentalsatz der Funktionentheorie [7, S.1196] ist eine Funktion $f(z)=$ $\xi(x, y)+i \eta(x, y)$ dann und nur dann auf einem Gebiet analytisch, wenn Real- und Imaginärteil von $f$ auf $\mathfrak{G}$ differenzierbar sind und dort die CAUCHY-RIEMAnNschen Differentialgleichungen, $\xi_{x}=\eta_{y}$ und $\xi_{y}=-\eta_{x}$, erfüllt werden. $\xi$ und $\eta$ sind in diesem Fall sogar konjugiert harmonische Funktionen, $\triangle \xi=\triangle \eta=0$. Für die Ableitung erhält man dann die Darstellung $f^{\prime}(z)=\xi_{x}+i \eta_{x}$.

\footnotetext{
${ }^{1}$ Aus dem Wegzusammenhang folgt der topologische Zusammenhang, $M$ kann dann nicht in zwei disjunkte nichtleere offene Mengen zerlegt werden. Die Umkehrung gilt im allgemeinen nicht.

${ }^{2}$ Damit wird eine Selbstüberschneidung ausgeschlossen, in diesem Fall heißt die Kurve auch einfach.

${ }^{3}$ Hier dient der Kurvensatz von Jordan als Grundlage, 17. S.235, Theorem 4.6f]

${ }^{4}$ Synonym verwendet werden die Begriffe regulär und holomorph. Von wesentlicher Bedeutung ist, daß es sich beim Definitionsbereich stets um ein Gebiet handelt.
} 


\subsection{Randwerte von CAUCHY-Integralen}

Ist $f(z)$ auf einem (nicht notwendiger Weise einfach zusammenhängenden) Gebiet $\mathfrak{G}$ analytisch, so existieren dort auch die Ableitungen $f^{(k)}(z), k=0,1,2 \ldots$, beliebig hoher Ordnung von $f$, die wiederum auf $\mathfrak{G}$ analytische Funktionen sind und es gilt für jede glatte, ganz in $\mathfrak{G}$ verlaufende Jordan-Kurve $\gamma_{0}$, in deren Innerem $f(z)$ analytisch ist, für jeden Punkt $z_{0}$ aus dem Inneren von $\mathfrak{C}_{0}=\gamma_{0}(t)$ mit $t \in\left[a_{0}, b_{0}\right]$ die CAUCHYsche Integralformel [7, S.1204, (37.19)]

$$
f^{(k)}\left(z_{0}\right)=\frac{k !}{2 \pi i} \oint_{\gamma_{0}} \frac{f(z)}{\left(z-z_{0}\right)^{k+1}} \mathrm{~d} z .
$$

Es ist genau zu beachten, daß $\mathfrak{C}_{0} \subset \mathfrak{G}$ gefordert ist und dabei keinerlei Aussagen über Randpunkte von $\mathfrak{G}$ getroffen werden. Die Integralformel von CAUCHY läßt sich jedoch hinsichtlich des Falles erweitern, bei dem $\mathfrak{G}$ durch $\mathfrak{C}$ berandet wird, das Kurvenintegral mithin über eine stückweise glatte Jordan-Kurve zu erstrecken ist, deren Bildmenge $\mathfrak{C}$ disjunkt zu $\mathfrak{G}$ ist. Will man also zu solchen Aussagen gelangen, bei denen das Bild $f\left(z_{0}\right)$ als Kurvenintegral über die Randkurve $\mathfrak{C}$ zu berechnen ist, so sind zusätzliche Annahmen zu treffen. Man findet [15, S.4ff.], [3, S.25f.] folgenden

Satz (Darstellung von $f$ mittels verallgemeinertem CAUCHYschen Integral).

Der Rand $\mathfrak{C}$ von $\mathfrak{G}$ sei gegeben durch eine stückweise glatte Jordan-Kurve $\gamma$. Ist $f(z)$ auf $\mathfrak{G}$ regulär und auf $\mathfrak{C} \cup \mathfrak{G}$ stetig $^{5}$, so gilt $\forall z_{0} \in \mathbb{C}$

1. Für $z_{0} \notin \mathfrak{C} \cup \mathfrak{G}$ ist

$$
\oint_{\gamma} \frac{f(z)}{\left(z-z_{0}\right)} \mathrm{d} z=0
$$

2. Für $z_{0} \in \mathfrak{G}$ ist

$$
f\left(z_{0}\right)=\frac{1}{2 \pi i} \oint_{\gamma} \frac{f(z)}{\left(z-z_{0}\right)} \mathrm{d} z
$$

3. Für $z_{0} \in \mathfrak{C}$ ist

$$
f\left(z_{0}\right)=\frac{1}{\alpha \pi i} \oint_{\gamma(H W)} \frac{f(z)}{\left(z-z_{0}\right)} \mathrm{d} z
$$

Man ersieht speziell, daß wegen (3.) auch für die Randpunkte eine Darstellung mittels Hauptwertintegral möglich wird. Mit $0<\alpha<2$ wird dort der durch $\pi$ dividierte Innenwinkel bezeichnet, für Punkte auf glatten Abschnitten von $\gamma$ gilt $\alpha=1$.

Die Untersuchung der Existenz von Ableitungen $f^{(k)}, k=1,2, \ldots$, holomorpher Funktionen und deren Darstellung an der Berandung, wie sie unter den Voraussetzungen des obigen Satzes für die Funktion $f$ selbst studiert wurde, stellt ein recht schwieriges Unterfangen dar. Jedenfalls sind gewisse Zusatzforderungen an das Verhalten von $f$ am Rand und die Eigenschaften von $\gamma \mathrm{zu}$ stellen. Daher wird an dieser Stelle nur auf die Ergebnisse von [33], [15, S.262ff., Anhang 4] und [3. S.11ff.] verwiesen, im Anlaßfall folgt an gegebener Stelle eine eingehendere Argumentation. Im Wesentlichen gilt, daß $f$ über einen analytischen Jordan-Bogen analytisch fortgesetzt werden kann.

Für den Spezialfall einer in $\mathfrak{E}$ regulären, auf $\overline{\mathfrak{E}}$ stetigen Funktion $f(x+i y)=\xi(x, y)+i \eta(x, y)$ gilt weiters die ScHWARZsche Integralformel [22, S.89ff., (9.2.12)], die es ermöglicht, die Funktionswerte für Punkte im Inneren des Einheitskreises aus dem Kurvenintegral über den Realteil am Rand des Kreises zu berechnen. Sie lautet in Polarform

$$
\rho e^{i \theta}=f\left(r e^{i \varphi}\right)=i \eta(0)+\frac{1}{2 \pi} \int_{0}^{2 \pi} \xi\left(e^{i \vartheta}\right) \frac{e^{i \vartheta}+r e^{i \varphi}}{e^{i \vartheta}-r e^{i \varphi}} \mathrm{d} \vartheta
$$

\footnotetext{
${ }^{5}$ Nach dem Fortsetzungssatz von CARAThÉodory ist die stetige Fortsetzbarkeit von $f$ sogar schon für geschlossene Jordan-Kurven ohne die gestellten Glattheitsforderungen erfüllt, 3, S.10]. Für nirgends differenzierbare JordanKurven (Fraktale bzw. Kochsche Kurven) wird aber der Begriff des Innenwinkels problematisch.
} 
Auf der Peripherie des Einheitskreises selbst läßt der Realteil von $f$ unter den selben Voraussetzungen wie oben eine Integraldarstellung des Imaginärteiles zu, es gilt auf Grund des Residuensatzes und der Identität $\left(e^{i \vartheta}+e^{i \varphi}\right) /\left(e^{i \vartheta}-e^{i \varphi}\right)=i \cot \frac{\varphi-\vartheta}{2}$ zwischen den konjugiert harmonischen Funktionen $\xi(x, y)$ und $\eta(x, y)$ dort der Zusammenhang [15, S.62, (1.1)]

$$
\eta\left(e^{i \varphi}\right)=\eta(0)+\frac{1}{2 \pi} \int_{0(H W)}^{2 \pi} \xi\left(e^{i \vartheta}\right) \cot \frac{\varphi-\vartheta}{2} \mathrm{~d} \vartheta
$$

\subsection{Die konforme Abbildung}

Sei nun die komplexe Funktion $f$ analytisch in einer Umgebung $K_{\varepsilon}\left(\zeta_{0}\right)$ eines Punktes $\zeta_{0}$ dergestalt gegeben, daß $f^{\prime}\left(\zeta_{0}\right) \neq 0$ gelte. Weiters seien dort $\zeta_{1}(t)$ und $\zeta_{2}(t)$ glatte Jordan-Bögen, die sich im Punkt $\zeta_{0}$ unter dem Winkel $\alpha=\lim _{\zeta_{1}, \zeta_{2} \rightarrow \zeta_{0}} \arg \frac{\zeta_{2}-\zeta_{0}}{\zeta_{1}-\zeta_{0}}$ schneiden mögen. Dann kann man zeigen, daß sich auch die Bildkurven $f\left(\zeta_{1}(t)\right)$ und $f\left(\zeta_{2}(t)\right)$ im Punkt $f\left(\zeta_{0}\right)$ unter dem selben Winkel $\alpha$ schneiden [25, S.149f.]. Auf Gebieten holomorphe Funktionen mit $\left|f^{\prime}(\zeta)\right|>0$ sind dort also winkeltreu.

Durch die Forderung $f^{\prime}(\zeta) \neq 0$ ist aber global auf einem Gebiet immer noch nicht gewährleistet, daß die Abbildung $f$ auch eindeutig umkehrbar ist. Dies liegt an der Tatsache, daß auf einem Gebiet analytische Funktionen mit nicht-verschwindender Ableitung nicht notwendigerweise injektiv sind, vergleiche dazu die Ausführungen in [3, S.4]. Auf einem Gebiet injektive holomorphe Funktionen heißen konform oder schlicht. Ob eine eindeutige Umkehrbarkeit erzielt werden kann, hängt wesentlich vom Verhalten der holomorphen Funktion bei Annäherung an den Gebietsrand und die dortigen Differenzierbarkeitseigenschaften von $f$ ab.

Eine interessante Feststellung läßt sich nun für den Fall der Regularität einer Abbildung in einer Umgebung $K_{\varepsilon}\left(\zeta_{0}\right)$ eines Punktes $\zeta_{0}$ treffen, in dem $f^{\prime}\left(\zeta_{0}\right)=0$ vorliegt. $\zeta_{0}$ heißt dann ein kritischer Punkt der Abbildung $f$ und es ist zu erwarten, daß der Winkel zweier sich in $\zeta_{0}$ schneidender Jordan-Bögen unter $f$ nicht erhalten bleibt. Es gilt [1, S.317ff., Theorem 5.3.1] folgender

Satz (Verhalten von $f$ in kritischen Punkten).

Sei $f$ analytisch in $\mathfrak{G}, f^{\prime}\left(\zeta_{0}\right)=f^{\prime \prime}\left(\zeta_{0}\right)=\cdots=f^{(k-1)}\left(\zeta_{0}\right)=0, f^{(k)}\left(\zeta_{0}\right) \neq 0$ für $\zeta_{0} \in \mathfrak{G}$. Dann wird der Winkel zweier sich im Punkt $\zeta_{0}$ schneidender Jordan-Bögen um das $k$-fache vergrößert.

Es ist zu erahnen, daß wir auf Grund der bisherigen Erkenntnisse mit numerischen Methoden versuchen werden, das Quadratgitter $\mathfrak{Q}_{n_{1}, n_{2}}$ einer konformen Abbildung zu unterwerfen. Den theoretischen Unterbau dazu liefert uns hinsichtlich Existenz und Eindeutigkeit folgender

Satz (RIEMAnNscher Abbildungssatz).

Gegeben seien nicht-leere, einfach zusammenhängende Gebiete $\mathfrak{G}_{1}$ und $\mathfrak{G}_{2}$ als echte Teilmengen der komplexen Zahlenebene und $z_{0} \in \mathfrak{G}_{1}, w_{0} \in \mathfrak{G}_{2}, \lambda \in \mathbb{R}$.

Dann existiert genau eine biholomorphe Abbildung $f: \mathfrak{G}_{1} \rightarrow \mathfrak{G}_{2}$ mit $f\left(z_{0}\right)=w_{0}$ und $\arg f^{\prime}\left(z_{0}\right)=\lambda$.

Insbesondere folgt daraus die Existenz einer konformen Abbildung eines einfach zusammenhängenden Gebietes auf das Innere des Einheitskreises $|w|<1$ als Normalgebiet und umgekehrt. Die Formulierung des Satzes stützt sich dabei auf HenRICI [17, S.380, Corollary 5.10c].

Damit steht der Konstruktion der konformen Abbildung vom Einheitsquadrat auf den Einheitskreis zumindest von der Theorie her nichts mehr im Wege. Da der RIEMAnnsche Abbildungssatz nicht konstruktiv ist, gilt es in den weiteren Ausführungen nach solchen konstruktiven Verfahren zu suchen. Das konkrete Problem der Transformation $\overline{\mathfrak{Q}} \rightarrow \overline{\mathfrak{E}}$ kann mit Hilfe von Polygonabbildungen in Angriff genommen werden, die eine spezielle Klasse konformer Abbildungen stellen und teils eine geschlossene Darstellung mittels elliptischer Funktionen ermöglichen. Um den Einheitskreis bzw. das transformierte Rechengitter konform weiter auf ein durch eine geschlossene Jordan-Kurve berandetes Gebiet abzubilden, bedienen wir uns speziell der Integralgleichung von THEODORSEN. Sie ist nur eine der numerisch konstruktiven Verfahren, die es gestattet, den Zusammenhang $\varphi \rightarrow \theta(\varphi)$ herzustellen. Bei Kenntnis dieser sogenannten Ränderzuordnungsfunktion ist die konforme Abbildung (und auch deren Ableitungen) dann auf dem gesamten Gebiet bekannt, vgl. Abschnitt 4.6. 


\subsection{Transformationen vom SchwARZ-Christoffel Typ}

Unabhängig voneinander fanden bereits um 1869 H.A. Schwarz und E.B. Christoffel, daß eine Abbildungsvorschrift, die es leistet, die obere komplexe Halbebene $\Im(w)>0$ bzw. das Innere des Einheitskreises $|w|<1$ konform auf ein durch einen geschlossenen, $n$-eckigen Polygonzug berandetes Gebiet abzubilden, durch

$$
f(w)=A+B \int_{0}^{w} \prod_{k=1}^{n}\left(1-\frac{\zeta}{w_{k}}\right)^{-\beta_{k}} \mathrm{~d} \zeta
$$

angegeben werden kann, 32. Die komplexen Konstanten $A$ und $B$ vermitteln dabei Translation bzw. Drehstreckung, die Punkte $w_{k}(k=1, \ldots n)$ liegen als Urbilder der Polygon-Eckpunkte $z_{k}$ auf der reellen Achse bzw. unimodular auf dem Einheitskreis und $\beta_{k}$ sind die zugehörigen, durch $\pi$ dividierten, äußeren Drehwinkel mit $\sum_{k=1}^{n} \beta_{k}=2$. Damit hat man folgende Aussage im

Satz (Schwarz-Christoffel Transformation von $|w|<1$ auf verallgemeinerten Polygonzug). Sei $\mathfrak{G}$ ein einfach zusammenhängendes Gebiet der komplexen Zahlenebene dergestalt, daß es durch einen Polygonzug mit Eckpunkten $z_{k}$ berandet wird. Ferner gelte für die zugehörigen Drehwinkel $-1 \leq \beta_{k}<1$ für endliches $z_{k}$ bzw. $1 \leq \beta_{k} \leq 3$ für $z_{k}=\infty$. Dann existiert mindestens eine analytische Funktion, die den Einheitskreis konform auf $\mathfrak{G}$ abbildet, wobei jede dieser Funktionen von der Gestalt (2.3) ist.

Die gewählten Formulierungen verwenden die Notationen in [31, Theorem 1] und [17, S.406, Theorem 5.12e]. Man findet zum einen in der Literatur auch Darstellungen, in denen die Innenwinkel verwendet werden [20, S.15, (1.2)], andererseits trifft man unter Beachtung von $\left(1-\zeta / w_{k}\right)^{-\beta_{k}}=$ $\left(-1 / w_{k}\right)^{-\beta_{k}}\left(\zeta-w_{k}\right)^{-\beta_{k}}$ vielfach auch [7, S.1291, (38.122)], [15, S.170, (3.1)] auf

$$
f(w)=C+D \int_{0}^{w} \prod_{k=1}^{n}\left(\zeta-w_{k}\right)^{-\beta_{k}} \mathrm{~d} \zeta .
$$

Daß die Bauart der Abbildung unabhängig davon ist, ob als Urbild die obere Halbebene oder der Einheitskreis zu Grunde gelegt wird kann man ersehen, indem man die Möbiustransformation

$$
z=i\left(\frac{1+w}{1-w}\right), \quad w=\frac{z-i}{z+i}, \quad \frac{\mathrm{d} z}{\mathrm{~d} w}=\frac{2 i}{(1-w)^{2}}
$$

betrachtet. Wie man unschwer erkennt, wird durch diese Vorschriften das Innere des Einheitskreises $|w|<1$ konform auf die obere Halbebene $\Im(z)>0$ abgebildet und umgekehrt, das Einsetzten in (2.3) unter Beachtung von $\sum_{k=1}^{n} \beta_{k}=2$ erbringt sofort das Behauptete (vgl. dazu [25, S.192f.], [17, S.404f.] oder auch [1, S.352]). Es ist im übrigen (durch Beachtung der Orientierung) auch möglich, Außengebiete einfach zusammenhängender Gebiete in analoger Weise abzubilden, [22, S.149ff.].

Um die Eindeutigkeit der Schwarz-Christoffelschen Abbildung im Sinne des RiEmanNschen Abbildungssatzes zu erzielen, hat man die Urbilder $w_{k}$ der Eckpunkte $z_{k}$ in geeigneter Weise zu bestimmen, das heißt, man steht vor einem Parameterproblem ${ }^{6}$. A priori festgelegt wurden bisher nur die äußeren Schwenkungswinkel $\beta_{k}$, die Konstanten $A$ und $B$ bestimmen dabei Lage und Größe des Polygonzuges, die Proportionen der Seitenlängen werden über die Lage der Urbilder (am Einheitskreis oder auf der reellen Achse) zueinander gesteuert und sind nur mit Einschränkungen frei wählbar. Ist die Abbildung vom Einheitskreis auf den Polygonzug eindeutig ermittelt worden, so gestattet die Bauart von (2.4) auf Grund der Biholomorphie auch die Angabe der Umkehrabbildung.

\footnotetext{
${ }^{6}$ Im Falle unregelmäßiger Polygone kann sich das Aufsuchen der Urbilder als komplexe Angelegenheit herausstellen, wir verweisen auf die im Literaturverzeichnis angegebenen Werke von GAIER [15, S.170ff.], TREFETHEN und Howell und erwähnen auch noch die für Matlab verfügbare Schwarz-Christoffel Toolbox [10, Driscoll].
} 


\subsection{Konforme Abbildung Einheitsquadrat auf Einheitskreis}

Die obigen Ausführungen versetzen uns in die Lage, das Innere des Einheitskreises konform auf das Innere des Einheitsquadrates abzubilden. Ausgehend von Gleichung (2.4) bestimmen sich die äußeren Drehwinkel $\beta_{k}$ in den vier Quadratecken $\left(z_{1}=1+i, z_{2}=-1+i, z_{3}=-1-i, z_{4}=1-i\right)$ sämtlich zu $\pi / 2$, sodaß die Abbildung angesetzt wird als

$$
z=f(w)=C+D \int_{0}^{w}\left(\zeta-w_{1}\right)^{-1 / 2}\left(\zeta-w_{2}\right)^{-1 / 2}\left(\zeta-w_{3}\right)^{-1 / 2}\left(\zeta-w_{4}\right)^{-1 / 2} \mathrm{~d} \zeta
$$

Im nächsten Schritt stellt man durch die Forderung $f(0)=0$ fest, daß die Translationskonstante $C$ entfällt und darüber hinaus kein Parameterproblem vorliegt, da man auf Grund der Symmetrien die unimodularen Urbilder sofort fixieren kann durch

$$
w_{k+1}=e^{i\left(\frac{\pi}{4}+\frac{k \pi}{2}\right)}, \quad k=0, \ldots 3 .
$$

Damit ist $f$ bereits bis auf eine Drehstreckung eindeutig bestimmt. Die verbleibende Konstante $D$ ermittelt man zweckmäßig mittels der Forderung $f(1)=1$. Es folgt daher [23, S.51ff.], [16, 6.1] das

Korollar (SchwARz-CHRISTOFFEL Transformation von $|w|<1$ auf das Einheitsquadrat). Die eindeutig festgelegte konforme Abbildung von $|w|<1$ auf das Innere des Einheitsquadrates ist mit der Setzung $D^{-1}=\int_{0}^{1} \mathrm{~d} \zeta / \sqrt{1+\zeta^{4}} \quad(\approx 1,0787052)$ über elliptische Funktionen gegeben durch

$$
z=f(w)=D \int_{0}^{w} \frac{\mathrm{d} \zeta}{\sqrt{1+\zeta^{4}}}=-\frac{2 \sqrt[4]{-1}}{\mathfrak{E}_{k ; \frac{1}{2}}} \mathfrak{E}_{f}[i \operatorname{arsinh}(\sqrt[4]{-1} w) ;-1]
$$

Auf Grund der Biholomorphie existiert im Quadrat auch die eindeutig festgelegte inverse Abbildung

$$
w=f^{-1}(z)=-\sqrt[4]{-1} \mathfrak{J}_{S n}\left[z \mathfrak{E}_{f}[i \operatorname{arsinh}(\sqrt[4]{-1}) ;-1] ;-1\right]
$$

Nähert man sich vom Inneren des Quadrates her seiner Berandung, so bleibt auch dort die Winkeltreue mit Ausnahme der Eckpunkte erhalten. Bis auf diese vier Ausnahmepunkte besitzt $f^{-1}(z)$ nämlich auch am Rand eine nichtverschwindende Ableitung. In den Eckpunkten gilt $f^{-1}\left(z_{k}\right)^{\prime}=0$ und $f^{-1}\left(z_{k}\right)^{\prime \prime} \neq 0$, nach den Aussagen des Satzes über das Verhalten einer holomorphen Funktion in kritischen Punkten schließt man daraus ${ }^{7}$, daß der Winkel in den Eckpunkten bei Abbildung auf den Einheitskreis in den Bildpunkten verdoppelt wird.

Ganz allgemein sind analytische Funktionen in jenen Punkten, in denen sie konform sind, zumindest in einer Umgebung um diese Punkte lokal umkehrbar und die dort existierenden Ableitungen dieser beiden Abbildungen zueinander reziprok [5, S.360], sodaß mit der Ableitung von (2.6) auch die Werte der Ableitung der Transformation des Quadrates auf den Kreis (mit Ausnahme der Eckpunkte) unmittelbar berechnet werden können. Dieser Umstand ist deshalb von Bedeutung, weil die Ableitungen im Zuge einer späteren Simulation verwendet werden und bereitzustellen sind.

Implementiert werden diese beiden Abbildungen und deren Ableitungen ${ }^{8}$ in Mathematica durch

$\mathrm{kq}\left[\mathrm{w}_{-}\right]:=-\left(2 *(-1)^{1 / 4} / \operatorname{EllipticK}[1 / 2]\right) * \operatorname{EllipticF}\left[\mathrm{I} * \operatorname{ArcSinh}\left[(-1)^{1 / 4} * \mathrm{w}\right],-1\right]$;

$\mathrm{qk}\left[\mathrm{z}_{-}\right]:=-(-1)^{1 / 4} \mathrm{JacobiSN}\left[\mathrm{z} * \operatorname{EllipticF}\left[\mathrm{I} * \operatorname{ArcSinh}\left[(-1)^{1 / 4}\right],-1\right],-1\right]$;

kqstrich [w_] :=kqstrich $[\mathrm{w}]=\mathrm{kq}^{\prime}[\mathrm{w}] ;$ qkstrich $\left[z_{-}\right]:=q k \operatorname{strich}[z]=\mathrm{qk}^{\prime}[\mathrm{z}]$;

\footnotetext{
${ }^{7}$ Diese Schlußfolgerung ist genau genommen unzulässig, da sie nach den Voraussetzungen des Satzes nur auf Punkte im Inneren, nicht aber auf solche auf der Berandung anwendbar ist. Durch eine mögliche analytische Fortsetzung von $f^{-1}$ über die Berandung hinaus können jedoch die vier nunmehr isolierten Singularitäten als innere Punkte eines neuen Gebietes so betrachtet werden, daß die Resultate des Satzes anwendbar werden.

${ }^{8}$ Als Argumente sind auch zweidimensionale Arrays komplexer Zahlen, mithin das gesamte Rechengitter zulässig.
} 

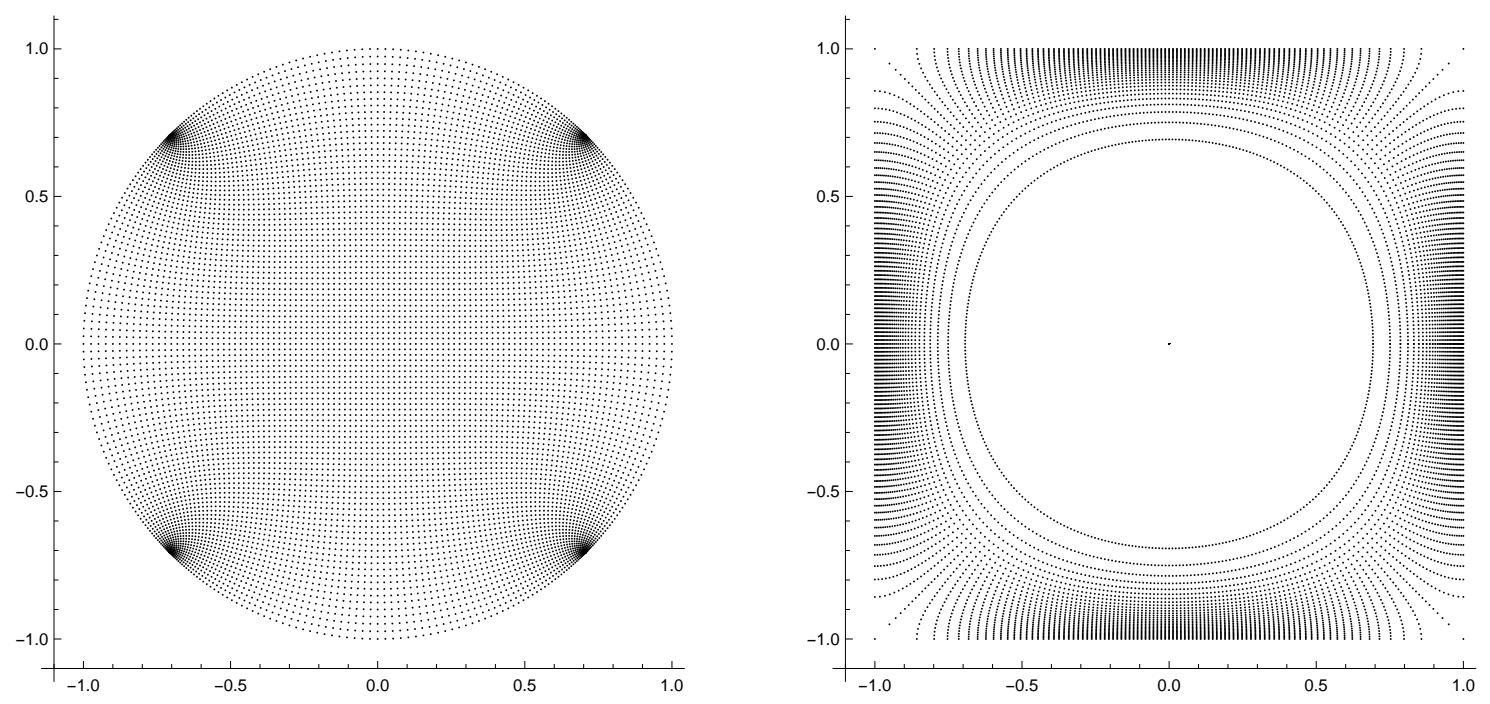

Abbildung 2.1: $\mathfrak{Q}_{101,101}$ (vgl. Abbildung 1.4 nach inverser Transformation 2.7) auf $\overline{\mathfrak{E}}$ (links). Ein Kreisgitter in Polarform nach Transformation (2.6) auf das Quadrat (rechts).

\subsection{Die Integralgleichung von THEODORSEN}

Nach der Ermittlung der konformen Abbildung des Quadrates auf den Kreis gehen wir an die Betrachtung der Abbildung $\overline{\mathfrak{E}} \mapsto \overline{\mathfrak{G}}$. Die Theorie stellt dazu einige konstruktive Verfahren zur Verfügung, die Grundidee besteht im Aufsuchen der sogenannten Ränderzuordnungsfunktion, eine eindimensionale reelle Aufgabenstellung, die den Winkel $\varphi$ am Einheitskreis mit dem Winkel $\theta(\varphi)$ am Gebiet in funktionalen Zusammenhang zu stellen trachtet. Dabei ist es auf Grund der Bijektivität natürlich unerheblich, in welche Richtung die Ränderzuordnung hergestellt wird, $\varphi(\theta)$ vom Abschluß des Gebietes $\overline{\mathfrak{G}}$ auf den Kreis führt auf lineare Integralgleichungen, während $\theta(\varphi)$ vom Kreis auf das Gebiet in nichtlinearen Hauptwertintegralgleichungen resultiert.

Da wir vom Quadrat kommend am Einheitskreis operieren, wollen wir aus Gründen der Bequemlichkeit die Ränderzuordnung $\theta(\varphi)$ aufsuchen. Die Berandung des Gebietes ist dabei jedoch gewissen Einschränkungen unterworfen, die Einfluß nehmen auf Existenz und Eindeutigkeit der Lösung. Sollten diese Voraussetzungen verletzt sein, so ist zur Konstruktion der Abbildung auf alternative Verfahren auszuweichen. Eine Übersicht über solche findet man in GAIER [15], an dem sich auch dieser Abschnitt orientiert.

Gleichung 2.2 gibt zunächst Anlaß dazu, reelle Funktionen der Form

$$
\mathcal{K}[f](\varphi)=f^{*}(\varphi):=\frac{1}{2 \pi} \int_{0(H W)}^{2 \pi} f(\vartheta) \cot \frac{\varphi-\vartheta}{2} \mathrm{~d} \vartheta
$$

näher zu studieren. Dabei sei $f$ eine stetige, $2 \pi$-periodische Funktion und die Stelle $\varphi=\vartheta$ führt bei der Auswertung des Integrals auf ein Hauptwertintegral. Die ebenfalls $2 \pi$-periodische Funktion $f^{*}$ wird als die zu $f$ konjugierte Funktion bezeichnet und der offensichtlich lineare Konjugiertenoperator $\mathcal{K}: f \mapsto f^{*}$ besitzt für $f \in \mathcal{L}_{p}(0,2 \pi)$ im Falle $p=2$ folgende Eigenschaften ${ }^{9}$ :

\footnotetext{
${ }^{9} \mathcal{L}_{p}(0,2 \pi)$ bezeichne wie üblich den Funktionenraum der $2 \pi$-periodischen, reellwertigen Funktionen, der vermöge $\|f\|_{p}:=\left(\frac{1}{2 \pi} \int_{0}^{2 \pi}|f(\xi)|^{p} \mathrm{~d} \xi\right)^{1 / p}$ normiert ist.
} 
- Hat die Fouriersche Reihe von $f$ die Gestalt

$$
f(\varphi)=\frac{a_{0}}{2}+\sum_{n=1}^{\infty}\left(a_{n} \cos n \varphi+b_{n} \sin n \varphi\right)
$$

dann erhält man als Fourier-Entwicklung von $f^{*}=\mathcal{K}[f]$

$$
f^{*}(\varphi)=\sum_{n=1}^{\infty}\left(a_{n} \sin n \varphi-b_{n} \cos n \varphi\right)
$$

die Relation der Fourier-Koeffizienten rechtfertigt demnach die Bezeichnung für $f^{*}$.

- Mit $f$ ist auch $f^{*}$ aus $\mathcal{L}_{2}(0,2 \pi)$ und es gilt ${ }^{10}$

$$
\|\mathcal{K}[f]\|_{2} \leq\|f\|_{2} \quad \forall f \in \mathcal{L}_{2}(0,2 \pi)
$$

- Sind $f, f^{\prime} \in \mathcal{L}_{2}(0,2 \pi)$ und ist $f$ absolut stetig ${ }^{11}$, so gelten diese Eigenschaften auch für $\mathcal{K}[f]$ und nach dem Lemma von SEIDEL ist

$$
\frac{\partial \mathcal{K}[f]}{\partial \varphi}=\mathcal{K}\left[\frac{\partial f}{\partial \varphi}\right]
$$

Insbesondere erhält man über konjugierte Funktionen einen Zusammenhang zwischen Real- und Imaginärteil einer in $|w|<1$ regulären und in $|w| \leq 1$ stetigen, komplexen Funktion $\zeta=f(w)=$ $\xi(u, v)+i \eta(u, v)$ auf der Peripherie des Einheitskreises. Der Imaginärteil $H(\varphi):=\eta(\cos \varphi, \sin \varphi)=$ $\Im\left[f\left(e^{i \varphi}\right)\right]$ gestattet dann nämlich — wieder ist für $\vartheta=\varphi$ der Cauchysche Hauptwert zu bilden — mit Hilfe des Realteiles $\Xi(\varphi):=\xi(\cos \varphi, \sin \varphi)=\Re\left[f\left(e^{i \varphi}\right)\right]$ eine Integraldarstellung der Form

$$
H(\varphi)=\eta(0,0)+\frac{1}{2 \pi} \int_{0(H W)}^{2 \pi} \Xi(\vartheta) \cot \frac{\varphi-\vartheta}{2} \mathrm{~d} \vartheta
$$

woraus man ersieht, daß es sich bei $\Xi^{*}(\varphi)=H(\varphi)-\eta(0,0)$ um die zu $\Xi(\varphi)$ konjugierte Funktion am Einheitskreis handelt.

Sei nun $\mathfrak{C}$ eine bezüglich des Ursprunges $\zeta_{0}=0$ der $\zeta$-Ebene sternförmige Jordankurve und $(\rho(\theta), \theta)$ ihre Darstellung in Polarkoordinaten. Dabei definieren wir die Sternförmigkeit eines von einer geschlossenen Kurve berandeten Gebietes $\mathfrak{G}$ der komplexen Zahlenebene bezüglich eines Punktes $\zeta_{0}$ aus dem Inneren von $\mathfrak{C}$ durch die Forderung

$$
\zeta_{0}+t\left(\hat{\zeta}-\zeta_{0}\right) \in \mathfrak{G}, \quad \forall t \in[0,1), \quad \forall \hat{\zeta} \in \mathfrak{C}
$$

Die Sternförmigkeit von $\mathfrak{G}$ bezüglich des Ursprungs ist eine für die Existenz und Eindeutigkeit der Ränderzuordnungsfunktion notwendige Einschränkung. Sie bedeutet anschaulich, daß jeder Randpunkt $\hat{\zeta}$ von $\mathfrak{C}$ derart mit dem Ursprung geradlinig verbindbar ist, daß dann auch die gesamte Verbindungsstrecke zur Gänze (mit Ausnahme des Punktes $\hat{\zeta}$ selbst) in $\mathfrak{G}$ liegen muß.

Gesucht ist nun die konforme Abbildung $\zeta=f(w)$ vom Inneren des Einheitskreises $|w|<1$ auf $\mathfrak{G}$, die hinsichtlich ihrer Eindeutigkeit durch die Forderungen $f(0)=0$ und $f^{\prime}(0)>0$ mit Normierungsvorschriften versehen wird. Die Funktion $f(w)$ ist auf $|w| \leq 1$ stetig und bildet den Einheitskreis bijektiv auf das abgeschlossene Gebiet $\overline{\mathfrak{G}}$ ab. Dabei entspricht jedem Randpunkt

\footnotetext{
${ }^{10}$ Diese Eigenschaft der Normkontraktion folgt aus der für jedes $f$ gültigen Parsevalschen Identität, $\frac{1}{\pi} \int_{0}^{2 \pi} f^{2}(\xi) \mathrm{d} \xi=\frac{a_{0}^{2}}{2}+\sum_{n=1}^{\infty}\left(a_{n}^{2}+b_{n}^{2}\right)=\frac{a_{0}^{2}}{2}+\frac{1}{\pi} \int_{0}^{2 \pi} f^{* 2}(\xi) \mathrm{d} \xi$.

$11 f$ heißt auf einem abgeschlossenen Intervall $[a, b]$ absolut stetig, wenn

$\forall \varepsilon>0 \quad \exists \delta>0: \quad \forall n \in \mathbb{N} \quad \forall a_{1}<b_{1} \leq a_{2}<b_{2} \leq \cdots \leq a_{n}<b_{n} \in[a, b]:$

$\sum_{i=1}^{n}\left(b_{i}-a_{i}\right) \leq \delta \Longrightarrow \sum_{i=1}^{n}\left|f\left(b_{i}\right)-f\left(a_{i}\right)\right| \leq \varepsilon$.

Sowohl stetige Differenzierbarkeit als auch Lipschitz-Stetigkeit von $f$ sind hinreichend für absolute Stetigkeit.
} 
$e^{i \varphi}$ ein Bildpunkt $\rho(\theta) e^{i \theta}$. Zur Herleitung der Gleichung für die Ränderzuordnungsfunktion $\theta(\varphi)$ betrachten wir die Hilfsfunktion

$$
F(w)=\ln \frac{f(w)}{w}=\xi(u, v)+i \eta(u, v) .
$$

$F(w)$ ist analytisch in $\mathfrak{E}$ und stetig auf $\overline{\mathfrak{E}}$, die Identität für den komplexen Logarithmus führt auf

$$
\ln \frac{f(w)}{w}=\ln |f(w)|-\ln |w|+i \arg (f(w))-i \arg (w),
$$

woraus man am Rand von $\mathfrak{E}$ bei Verwendung von Polarkoordinaten sofort

$$
\begin{aligned}
\Xi(\varphi) & =\xi(\cos \varphi, \sin \varphi)=\ln \rho(\theta(\varphi)), \\
H(\varphi) & =\eta(\cos \varphi, \sin \varphi)=\theta(\varphi)-\varphi
\end{aligned}
$$

folgert. Nun ist aber nach 2.11) $H(\varphi)-\eta(0,0)$ die zu $\Xi(\varphi)$ konjugierte Funktion und da nach Voraussetzung $\eta(0,0)=0$ gilt, erhält man die gesuchte Integralgleichung zur Ermittlung der Ränderzuordnungsfunktion $\theta(\varphi)$ durch

$$
\theta(\varphi)=\varphi+\frac{1}{2 \pi} \int_{0(H W)}^{2 \pi} \ln \rho(\theta(\vartheta)) \cot \frac{\varphi-\vartheta}{2} \mathrm{~d} \vartheta=\varphi+\mathcal{K}[\ln \rho(\theta)](\varphi) .
$$

Die nach TheOdorsen benannte Gleichung (2.14) ist nichtlinear und singulär, an der Stelle $\vartheta=\varphi$ ist der Cauchysche Hauptwert zu ermitteln. Daß die Theodorsensche Integralgleichung mindestens eine Lösung besitzt, kann aus dem Riemannschen Abbildungssatz geschlossen werden. Zur Sicherstellung der Eindeutigkeit einer solchen Lösung muß noch eine zusätzliche Forderung an die Berandung, die sogenannte $\varepsilon$-Bedingung, gestellt werden ${ }^{12}$, die dann zusammen mit der Sternförmigkeit des Gebietes für den Fall $0<\varepsilon<1$ als Kreisnähe bezeichnet wird. Damit [15, S.66, Satz 1.3] gilt

Satz (Existenz und Eindeutigkeit der Lösung der TheOdorsEnschen Integralgleichung). Erfüllt $\mathfrak{C}$ eine $\varepsilon$-Bedingung mit $\varepsilon<1$, so hat Gleichung (2.14) genau eine stetige Lösung.

Ist die Ränderzuordnungsfunktion $\theta(\varphi)$ gefunden, so bieten sich zur Fortsetzung ins Innere des Einheitskreises mehrere Möglichkeiten. Für die Fourier-Entwicklungen der konjugierten Funktionen $\Xi(\varphi)$ und $H(\varphi)$ gilt nach 2.9 und 2.10 zunächst

$$
\Xi(\varphi)=\frac{a_{0}}{2}+\sum_{k=1}^{\infty} a_{k} \cos k \varphi+b_{k} \sin k \varphi \quad \text { und } \quad H(\varphi)=\sum_{k=1}^{\infty} a_{k} \sin k \varphi-b_{k} \cos k \varphi,
$$

mit den Fourier-Koeffizienten

$$
a_{k}=\frac{1}{\pi} \int_{0}^{2 \pi} \ln \rho(\theta(\tau)) \cos (k \tau) \mathrm{d} \tau \quad \text { und } \quad b_{k}=\frac{1}{\pi} \int_{0}^{2 \pi} \ln \rho(\theta(\tau)) \sin (k \tau) \mathrm{d} \tau .
$$

Der Umstand der Regularität ${ }^{13}$ der im Inneren des Einheitskreises gesuchten Hilfsfunktion aus (2.12 bedingt nun, daß es sich bei Real- und Imaginärteil von $F(z)$ um harmonische Funktionen $\xi(r, \varphi)$ und $\eta(r, \varphi)$ handeln muß. Damit hat man ein DiRichletsches Randwertproblem mit den Funktionen aus (2.15) als vorzuschreibende Randbedingungen vorliegen,

$$
\triangle \xi(r, \varphi)=\triangle \eta(r, \varphi)=0 ; \quad \xi(1, \varphi)=\Xi(\varphi), \quad \eta(1, \varphi)=H(\varphi) .
$$

Der Lösungsweg führt über den Separationsansatz auf eine Differentialgleichung vom EuLERschen Typ [7, S.1167f.] und erbringt

$$
\xi(r, \varphi)=\frac{a_{0}}{2}+\sum_{k=1}^{\infty} r^{k}\left(a_{k} \cos k \varphi+b_{k} \sin k \varphi\right), \quad \eta(r, \varphi)=\sum_{k=1}^{\infty} r^{k}\left(a_{k} \sin k \varphi-b_{k} \cos k \varphi\right) .
$$

\footnotetext{
${ }^{12}$ Eine bezüglich des Ursprunges sternförmige Jordankurve $\mathfrak{C}$ erfüllt eine $\varepsilon$-Bedingung, wenn $\rho(\theta)$ in $[0,2 \pi]$ absolut stetig ist und weiters $|\sigma(\theta)|=\left|\frac{\rho^{\prime}(\theta)}{\rho(\theta)}\right| \leq \varepsilon$ für fast alle $\theta \in[0,2 \pi]$ gilt.

${ }^{13}$ Der Ursprung gibt nach Anwendung der Regel von Marquis DE L'Hospital keinen Anlaß zur Sorge, da nach Voraussetzung $f^{\prime}(0)>0$ ist.
} 
Ruft man sich in Erinnerung, daß es sich bei diesen beiden Lösungsfunktionen um den Real- bzw. Imaginärteil der in 2.12 eingeführten Hilfsfunktion $F(w)$ handelt, so ergibt sich nach entsprechender Umformung das Bild eines Punktes $w=r e^{i \varphi}$ mit $|w| \leq 1$ in Polardarstellung zu

$$
\begin{aligned}
f\left(r e^{i \varphi}\right)= & r \exp \left\{\frac{a_{0}}{2}+\sum_{k=1}^{\infty} r^{k}\left(a_{k} \cos k \varphi+b_{k} \sin k \varphi\right)\right\} \\
& \times \exp \left\{i\left[\varphi+\sum_{k=1}^{\infty} r^{k}\left(a_{k} \sin k \varphi-b_{k} \cos k \varphi\right)\right]\right\}=\rho e^{i \theta}
\end{aligned}
$$

In dieser Gleichung stellt der erste Faktor das Bild $\rho(\theta)$ des Radiusvektors $r$ dar, wohingegen man den Ausdruck in der eckigen Klammer als Bildwinkel $\theta(\varphi)$ identifiziert. Die kartesische Darstellung findet man nach kurzem Rechengang, es ist [7, S.1303]

$$
\zeta=f(w)=w \exp \left[\frac{a_{0}}{2}+\sum_{k=1}^{\infty}\left(a_{k}-i b_{k}\right) w^{k}\right] .
$$

Alternativ dazu läßs sich die gesuchte Abbildung $f(w)$ in $\mathfrak{E}$ mit der ScHwarzschen Formel (2.1) darstellen, es gilt nach [15, S.65, (1.9)]

$$
\zeta=f(w)=w \exp \left[\frac{1}{2 \pi} \int_{0}^{2 \pi} \ln \rho(\theta(\vartheta)) \frac{e^{i \vartheta}+w}{e^{i \vartheta}-w} \mathrm{~d} \vartheta\right] .
$$

Auch eine direkte Auswertung mittels CAUCHYscher Integralformel ist möglich, setzt man darin die Integrationsvariable am Einheitskreis unimodular an mit $w=e^{i \vartheta}$, so wird $\mathrm{d} e^{i \vartheta}=i e^{i \vartheta} \mathrm{d} \vartheta$ und mit 2.13 gelangt man bei bekannter Ränderzuordnung $\theta(\varphi) \mathrm{zu}$

$$
\zeta=f(w)=w \exp \left[\frac{1}{2 \pi} \int_{0}^{2 \pi} \frac{\ln \rho(\theta(\vartheta))+i(\theta(\vartheta)-\vartheta)}{1-w e^{-i \vartheta}} \mathrm{d} \vartheta\right]
$$

Die reichlich theoretischen Aussagen dieses Kapitels sollen als eine Zusammenstellung der für die konforme Abbildung benötigten Formeln fungieren, sie lassen sich für unsere Zwecke wie folgt zusammenfassen. Zunächst ist festzuhalten, daß die konforme Abbildung von $\mathfrak{Q}_{n_{1}, n_{2}}$ auf den Einheitskreis Mathematica durch Verwendung elliptischer Funktionen (namentlich der JACOBIschen elliptischen Funktion Sinus Amplitudinis und des unvollständigen elliptischen Integrals erster Art) vor keinerlei Probleme stellt, und zwar weder hinsichtlich Auswertungsgeschwindigkeit noch was die (beliebig vorgebbare) Genauigkeit anlangt. Dies gilt auch für die Ableitungen dieser Funktionen.

Für die weitere Abbildung des Einheitskreises auf ein allgemeines Gebiet muß die Ränderzuordnungsfunktion durch Lösen der Theodorsengleichung (2.14) möglichst genau bereitgestellt werden. Bei Kreisnähe und hinreichenden Glattheitseigenschaften der Berandung ist dabei die FourierEntwicklung von $\ln \rho(\theta(\varphi))$ das Mittel der Wahl, da bei Implementierung über (2.16) darüberhinaus auch sofort die Ableitungen der Funktion auswertbar sind. Bei nur stückweise glatter Randkurve oder Verletzung der Kreisnähe führt die Verwendung der Fourier-Koeffizienten zu keinem zufriedenstellenden Ergebnis mehr, wurde die Ränderzuordnungsfunktion auf andere Weise berechnet (in Abschnitt 3.3 etwa wird die Abbildung Kreis auf Quadrat durch kontinuierliche Fixpunktiteration des Hauptwertintegrals in Angriff genommen), so kann man für die Auswertungen auf Formel (2.17) oder 2.18 zurückgreifen. Die Vorgehensweise dazu wird in Abschnitt 4.6 dargelegt.

Den praktischen Aspekten der Konstruktion der Abbildung widmet sich das nächste Kapitel. 


\title{
Kapitel 3
}

\section{Praxis der konformen Abbildung}

\author{
It is generally preferable to keep the singularity and deal with it correctly \\ Rather than disguise it and hope for the best... [28]
}

Die Literatur zur numerischen Behandlung der konformen Abbildung ist wie die Zahl der zur Verfügung stehenden Verfahren mannigfaltig. Es soll hier weniger der Versuch unternommen werden, einen Überblick über die Methoden zu geben, sondern vielmehr an Hand dreier ausgewählter Beispiele demonstriert werden, wie unter Zuhilfenahme von Mathematica die Fixpunktiteration und das Verfahren von NEWTON dergestalt implementiert werden können, daß daraus eine hochgenaue Approximation der Ränderzuordnungsfunktion und damit der gesuchten konformen Abbildung sowie deren Ableitungen resultiert. Eine möglichst hohe Genauigkeit ist deshalb notwendig, weil zu späterem Zeitpunkt die Ableitungen der konformen Abbildung als metrische Größen in die Simulationsmodelle einfließen werden und in diesem Umstand die Hauptfehlerquelle der Simulation begründet liegt. Somit steht und fällt die Gesamtsimulation mit der Güte dieser Näherung.

Es ist naheliegend, die Implementierung der Iteration der Theodorsengleichung (2.14) mit einem Standardproblem anzugehen, bei dem die exakte Abbildung zu Vergleichszwecken bekannt ist. Ein solches liegt in Form der am Einheitskreis gespiegelten Ellipse vor, Ränderzuordnungsfunktion und konforme Abbildung sind für diesen Fall in geschlossener Form verfügbar.

Des weiteren bietet sich die numerische Konstruktion der inversen Abbildung vom Kreis auf das Einheitsquadrat an: Hier ist zum einen die exakte Transformation ebenfalls bekannt, zweitens kann damit die Vorgehensweise zur Konstruktion von Polygonabbildungen bzw. von Gebieten mit Ecken demonstriert werden und schließlich läßt sich dadurch ein pathologisches Szenario entwickeln, in dem an Stelle der direkten Simulation am Quadrat aus Kapitel 1 der Einheitskreis als Normalgebiet zwischengeschaltet wird, von dem aus auf ein kreisnahes Gebiet mit $\varepsilon$-Bedingung weiter abgebildet wird. Durch eine solche Vorgehensweise wird der Fehler der noch durchzuführenden Simulation einem Vergleich mit den Ergebnissen aus Abschnitt 1.4 zugänglich.

Als drittes Gebiet wird eine bezüglich der $\eta$-Achse symmetrische Teilmenge betrachtet und deren Gestalt mit Hinblick auf die $\varepsilon$-Bedingung variiert. Die Berandung $\rho(\theta)$ wird zur Erzielung der notwendigen Glattheitseigenschaften durch FoURIER- bzw. HERMITE-Interpolation vorgegeben und daraus über die Ränderzuordnungsfunktion die konforme Abbildung konstruiert. Auf diesem Gebiet soll dann im abschließenden Kapitel eine realistische, dreidimensionale Simulationsstudie mit dem Einheitsquadrat als Trägergeometrie durchgeführt werden.

Mathematica ist auch hinsichtlich der Evaluierung nichtlinearer singulärer Integrale ein überaus leistungsfähiges Werkzeug, das allerdings dem Benutzer in Bezug auf die Einstellung von Optionen und Methoden (Auswahl der geeigneten Integrationsroutine, Feinjustierung der anzugebenden Parameter und ähnliches mehr) einiges an Erfahrung abverlangt. Diese Leistungsfähigkeit führt zur Erkenntnis, daß bei den Iterationsvorschriften zur Gewinnung der Ränderzuordnungsfunktion abseits der klassischen Fourier-Entwicklung — der Konjugiertenoperator $\mathcal{K}[f]$ auch durch direkte Auswertung, wenn auch nicht immer mit vertretbarem zeitlichen Aufwand, gewonnen werden kann. 


\subsection{Numerische Behandlung der Gleichung von THEODORSEN}

Zeitlos und grundlegend ist die Arbeit von GaIER [15], HüBner [21] entwickelt in seiner Publikation aus dem Jahre 1983 ein Verfahren, das als eine Verallgemeinerung des NEWTonschen Verfahrens für die gesuchte Lösungsfunktion der Theodorsengleichung verstanden werden kann und RICHARDSON [28] demonstriert 2004, wie mit Mathematica das Abbildungsproblem mit großer Genauigkeit in den Griff zu bekommen ist. Auch das Werk von KYTHE [23] soll hier noch Erwähnung finden.

\subsubsection{Die Methode der sukzessiven Approximation}

Versucht man, die Theodorsensche Integralgleichung 2.14 durch Fixpunktiteration anzugehen, so ist zunächst zu beachten, daß es sich bei dem gesuchten „Fixpunkt" $\theta(\varphi)$ um eine monoton wachsende, reelle Funktion handelt und somit eine funktionale Fixpunktgleichung in der Form

$$
\theta=\iota+\mathcal{K}[\ln \rho(\theta)]=\mathcal{F}(\theta)
$$

vorliegt. Dabei werde mit $\iota(\varphi)=\varphi$ die identische Funktion bezeichnet und die Bildungsvorschrift zur Fixpunktiteration erhält mit einer geeigneten Startfunktion $\theta_{0}$ die Gestalt

$$
\theta_{n+1}=\mathcal{F}\left(\theta_{n}\right), \quad n=0,1,2, \ldots,
$$

wobei man — sofern keine bessere Alternative als Startfunktion zur Verfügung steht — das Verfahren mit $\theta_{0}=\iota$ starten kann. Unter der Annahme, daß auf dem Gebiet keine weiteren Symmetrien vorliegen, bieten sich bei Unterteilung des Einheitskreises in $2 N$ äquidistante Stützstellen $\varphi_{j}=\frac{j \pi}{N}, j=0, \ldots 2 N-1$, zur Implementierung nun zwei Varianten:

- Nach trigonometrischer Interpolation von $\ln \rho\left(\theta_{n}\right)$ an den $2 N$ Stellen $\varphi_{k}$ ist $\mathcal{K}\left[\ln \rho\left(\theta_{n}\right)\right]$ durch 2.10 unmittelbar berechenbar. Damit kann sofort auch $\theta_{n+1}(\varphi)$ als kontinuierliche Funktion gebildet werden und das Verfahren läuft. Die diskrete Fourier-Interpolation vom Grad $N$ bietet sich deshalb an, weil mit $\rho(\theta)$ auch $\ln \rho(\theta(\varphi)) 2 \pi$-periodisch ist, zu beachten ist, daß bei einer geraden Anzahl von äquidistanten Stützstellen der Koeffizient $b_{N}$ verschwindet, also

$$
\ln \rho(\theta(\varphi)) \approx \frac{a_{0}}{2}+\sum_{j=1}^{N-1}\left(a_{j} \cos j \varphi+b_{j} \sin j \varphi\right)+\frac{a_{N}}{2} \cos N \varphi .
$$

- Läßt man das Hauptwertintegral in 2.14 von Mathematica direkt an den Stützstellen $\varphi_{k}$ berechnen $^{1}$, so muß das Ergebnis noch mit einer geeigneten Routine interpoliert werden, um zu einer kontinuierlichen Funktion und damit zur nächsten Iterierten $\theta_{n+1}(\varphi)$ zu gelangen. Vom Modul Interpolation [] werden allerdings nur Spline- und Hermite-Interpolation (beliebigen Grades) angeboten, wobei nur letztere eine Option zur periodischen Interpolation bietet.

Die direkte Auswertung zeichnet sich vor allem durch eine hohe, über NIntegrate [] steuerbare Genauigkeit aus. Eine anschließende Verwendung der Funktion Fit [] kann zur Interpolation ebenfalls in Erwägung gezogen werden, weil sie die Angabe von Basisfunktionen unterstützt, mit Hilfe derer ein Kleinstquadrateausgleich bewerkstelligt wird. Für den Fall, daß die Anzahl der Stützstellen mit jener der Basisfunktionen übereinstimmt, führt dies nämlich auf eine gewöhnliche interpolierende Funktion zurück. Damit kann auch die trigonometrische Interpolation implementiert werden.

Nun stellt sich aber heraus, daß die angeführten Interpolationsroutinen hinsichtlich der Genauigkeit insofern limitiert sind, als die Koeffizienten der Interpolierenden von Mathematica intern mit \$MachinePrecision (das sind im allgemeinen 16 Nachkommastellen) beschränkt werden. Um diesen Sachverhalt zu umgehen, verwenden wir wo irgend möglich zur Interpolation von Funktionen die Routine LinearModelFit [], die analog zur numerischen Integration die Angabe einer WorkingPrecision per Option gestattet, mit Hilfe derer die Koeffizienten der Interpolierenden dann mit der entsprechenden Genauigkeit zur Verfügung gestellt werden können.

\footnotetext{
${ }^{1}$ NIntegrate $\left[\log [r[\right.$ thetaneu $[y]]] * \operatorname{Cot}\left[\left(\right.\right.$ phi_k-y)/2] , $\left\{\mathrm{y}, 0, \mathrm{phi} \_\mathrm{k}, 2 \mathrm{Pi}\right\}$, MaxPoints $\rightarrow \mathrm{mp}$, MinRecursion $\rightarrow \mathrm{mn}$,

MaxRecursion $\rightarrow \mathrm{mx}$, WorkingPrecision $\rightarrow \mathrm{w}$, Method $\rightarrow$ "PrincipalValue", AccuracyGoal $\rightarrow$ a, PrecisionGoal $\rightarrow \mathrm{p}]$
} 


\subsubsection{Das verallgemeinerte Newtonverfahren}

Vom NEWTONschen Verfahren zur Nullstellensuche reeller Funktionen ist bekannt, daß im Falle der Konvergenz diese quadratisch ist. Da für das oben besprochene Verfahren der sukzessiven Approximation nur lineare Konvergenzordnung vorliegt, wäre eine Erweiterung der klassischen Newtoniteration auf Funktionen, um damit die Theodorsengleichung behandeln zu können, aus numerischer Sicht überaus interessant. Um Gleichung (2.14) als funktionales Nullstellenproblem umzuformulieren, wird dazu die Funktion $\psi(\varphi)=\theta(\varphi)-\varphi$ eingeführt, sodaß daraus mit $\psi=\mathcal{K}[\ln \rho(\psi+\iota)]$

$$
\mathcal{F}(\psi)=\psi-\mathcal{K}[\ln \rho(\psi+\iota)] \equiv 0
$$

resultiert [23, S.235]. Rein formal lautet dann die Bildungsvorschrift eines Iterationsschrittes

$$
\psi_{n+1}=\psi_{n}-\left[\mathcal{F}^{\prime}\left(\psi_{n}\right)\right]^{-1} \mathcal{F}\left(\psi_{n}\right), \quad n=0,1,2, \ldots,
$$

wobei die $\psi_{n}$ diesmal periodisch mit $2 \pi$ sind und die Iteration mit der identisch verschwindenden Funktion $\psi_{0} \equiv 0$ gestartet werden kann. Da man vom Newtonverfahren jedoch weiß, daß ihm nahe an der Nullstelle gelegene Startwerte sehr entgegenkommen, wird man bei gegebenenfalls auftretenden Konvergenzproblemen eine Enditeration aus der Fixpunktiteration als Startfunktion heranziehen.

Die Natur des Operators $\mathcal{F}$ liegt dabei noch völlig im Dunkeln, HüBNER untersucht ihn in [21], bedient sich dabei zur Darstellung von $\mathcal{F}^{\prime}$ der FRÉCHET-Ableitung und zeigt, daß der dazu inverse Operator durch Lösen eines RiEMAnN-HilberTschen Problems gewonnen und in geschlossener Form dargestellt werden kann. Bei Vorliegen eines sternförmigen Gebietes und unter der Voraussetzung, daß $\sigma^{\prime}$ (mit $\sigma=\rho^{\prime} / \rho$ ) beschränkt und $2 \pi$-periodisch ist, ferner die $n$-te Iterierte $\psi_{n}(\varphi)$ einem Sobolev-Raum angehört ${ }^{2}$, gibt er in [21, S.24, Theorem 4] folgende Bildungsvorschrift zur Gewinnung von $\psi_{n+1}(\varphi)$, die das Verfahren auch in kontinuierlicher Form implementierbar macht:

$$
\psi_{n+1}=-\widetilde{r} \sin \beta \cos \beta+e^{\mathcal{K}[\beta]} \cos \beta\left(\mathcal{K}\left[\widetilde{r} \cos \beta e^{-\mathcal{K}[\beta]}\right]+\widetilde{C}\right),
$$

wobei

$$
\begin{aligned}
& \beta(\varphi)=\arcsin \frac{\sigma\left(\psi_{n}(\varphi)+\varphi\right)}{\sqrt{1+\sigma^{2}\left(\psi_{n}(\varphi)+\varphi\right)}}, \\
& \widetilde{r}=-\psi_{n} \sigma\left(\psi_{n}+\iota\right)+\ln \rho\left(\psi_{n}+\iota\right)
\end{aligned}
$$

und

$$
\widetilde{C}=\frac{\int_{0}^{2 \pi}\left\{\widetilde{r} \sin \beta \cos \beta-e^{\mathcal{K}[\beta]} \cos \beta \mathcal{K}\left[\widetilde{r} \cos \beta e^{-\mathcal{K}[\beta]}\right]\right\} \mathrm{d} \varphi}{\int_{0}^{2 \pi} e^{\mathcal{K}[\beta]} \cos \beta \mathrm{d} \varphi}
$$

gesetzt ist. Bei Kenntnis der exakten Ränderzuordnung $\theta(\varphi)$ gilt für den Fehler $e_{n}$ die Darstellung

$$
e_{n}(\varphi)=\theta(\varphi)-\psi_{n}(\varphi)-\varphi
$$

Wir fügen noch an, daß für das Verfahren zwar gefordert wird, daß $\rho(\theta)$ einer $\varepsilon$-Bedingung genügt, die Bedingung $\varepsilon<1$ jedoch fallengelassen werden kann. Dies macht das HüBNERsche Verfahren zusätzlich interessant, da damit eine größere Klasse von Gebieten behandelt werden kann. Für die Varianten zur numerischen Auswertung des Konjugiertenoperators $\mathcal{K}$ gelten im übrigen die selben Bemerkungen wie im vorhergehenden Abschnitt zur sukzessiven Approximation.

Weiterführende Untersuchungen zur Konvergenz des Verfahrens finden sich bei WEGMANN [34, wo auch alternative diskrete Verfahren vorgestellt und analysiert werden. Wie sich herausstellen wird, erbringt eine dort etwas umformulierte Anwendung der diskreten HüBNERschen Variante die besten Ergebnisse hinsichtlich Rechengeschwindigkeit bei hoher Approximationsgenauigkeit.

\footnotetext{
${ }^{2}$ Gefordert wird damit die Existenz der (dann eindeutig bestimmten) schwachen Ableitung $\hat{\psi}_{n}$ von $\psi_{n}$, $\forall \tau \in C^{\infty}(0,2 \pi): \int_{0}^{2 \pi} \hat{\psi}_{n}(\vartheta) \tau(\vartheta) \mathrm{d} \vartheta=-\int_{0}^{2 \pi} \psi_{n}(\vartheta) \tau^{\prime}(\vartheta) \mathrm{d} \vartheta$.
} 


\subsection{Iteration der gespiegelten Ellipse}

Für die am Einheitskreis gespiegelte Ellipse $(0<p<1)$ kann die Berandung mittels

$$
\rho(\theta)=\sqrt{1-\left(1-p^{2}\right) \cos ^{2} \theta}
$$

beschrieben werden, exakte konforme Abbildung und Ränderzuordnung ergeben sich zu [15, S.264]

$$
\zeta=f(w)=\frac{2 p w}{1+p+(1-p) w^{2}} \quad \text { und } \quad \theta(\varphi)=\arctan (p \tan \varphi) .
$$

Für $p=6 / 10$ ist $\max |\sigma(\theta)|=8 / 15<1$, das Gebiet weist also Kreisnähe auf und auf Grund der Glattheitseigenschaften der Berandung sind keine Probleme für die Iterationsverfahren zu erwarten.

Obwohl im vorliegenden Fall wegen der vorhandenen Symmetrien sämtliche zur Konstruktion notwendigen Informationen aus dem Intervall $\left[0, \frac{\pi}{2}\right]$ gewonnen werden können, werden wir im Hinblick auf größere Flexibilität das Intervall $[0,2 \pi]$ für die trigonometrische Interpolation zu Grunde legen. Dies garantiert eine leichtere Adaptierbarkeit des Codes auf andere Geometrien. Sehr wohl zu beachten und für eine korrekte Implementierung wesentlich ist eine sorgfältige Untersuchung der Symmetrien, um aus diesen Information über die Gestalt der Fourierkoeffizienten zu gewinnen.

Das Verfahren der sukzessiven Approximation schwingt sich bei RichARDson [28, S.470] bei einem Fehler von ca. $2 \cdot 10^{-8}$ ein. Er wertet dabei den Konjugiertenoperator direkt mittels NIntegrate an den 20 Stützstellen aus und interpoliert anschließend per Fit mit trigonometrischen Polynomen der Form $\sin 2 k \varphi$ — da es sich bei $\ln \rho(\theta(\varphi))$ um eine gerade Funktion handelt, resultiert nämlich aus der Konjugierten eine reine Sinus-Reihe, durch die Schiefsymmetrie bezüglich $\pi / 2$ verschwinden zudem auch die ungeraden Summanden.

Wir haben das Verfahren der sukzessiven Approximation mit $2 n=256$ Stützstellen (aus den bereits erwähnten Argumenten hinsichtlich der Genauigkeit mittels der Routine LinearModelFit) durch direkte Entwicklung von $\ln \rho(\theta(\varphi))$ in eine Cosinus-Reihe $\sum \cos 2 k \varphi$ implementiert, die Konjugierte errechnet sich dann für jedes $\varphi \in[0,2 \pi]$ ohne nennenswerten Rechenaufwand aus $(2.10)$. Das Verfahren konvergiert dabei relativ langsam (ein Iterationsschritt erbringt eine Halbierung des Fehlers), nach 100 Iterationen gelangt man in die Region 5.10 ${ }^{-30}$, vgl. Abbildung 3.1. Darunter angeführt ist der Code, der als Ausgangspunkt für die weiteren Implementierungen dient.

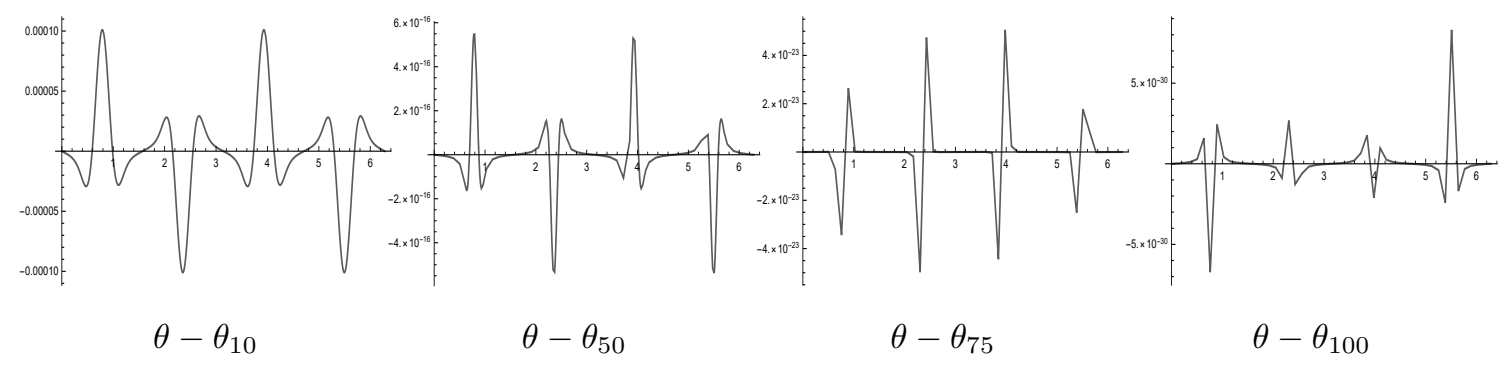

Abbildung 3.1: Der Fehler bei Iteration der Ränderzuordnung mittels sukzessiver Approximation.

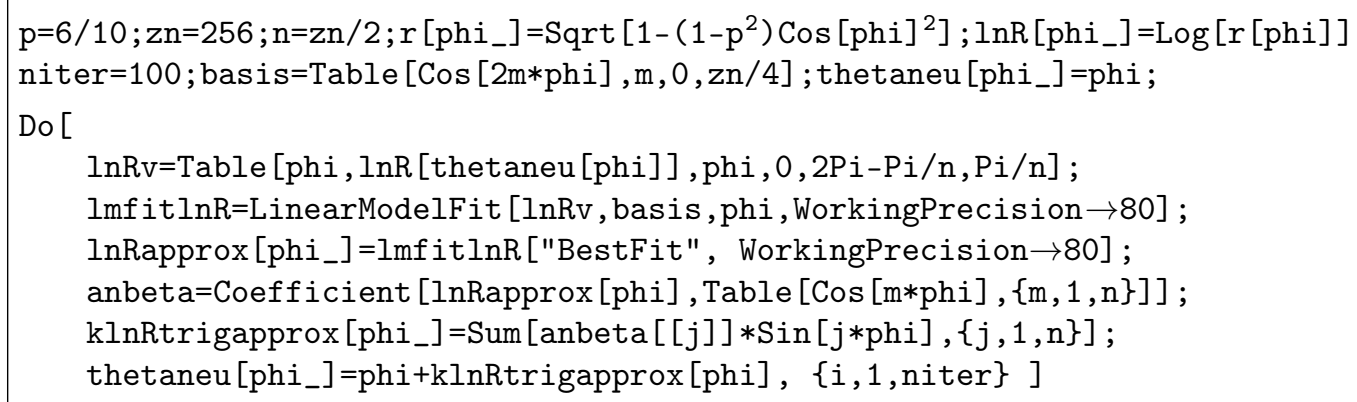


Vergleicht man das Verfahren der sukzessiven Approximation mit dem Newtonschen Verfahren, so wird der dabei entstehende Mehraufwand und die Fehleranfälligkeit bei Implementierung durch eine rasante Konvergenzgeschwindigkeit mehr als ausgeglichen. Die zur Lauffähigkeit einer Iterationsschleife notwendigen Schritte seien nachfolgend kurz zusammengestellt, sie lauten (vgl. Abbildung 3.2 für die gespiegelte Ellipse mit $N=2 n$ Stützstellen $\varphi_{k}=\frac{k \pi}{n}, k=0, \ldots N-1$ :

- An den Stützstellen werden zunächst die Werte $\beta\left(\varphi_{k}\right)$ berechnet und festgestellt, daß es sich bei $\beta$ um eine bezüglich des Ursprunges und $\pi / 2$ ungerade Funktion handelt. Man verwendet daher zur Interpolation von $\beta$ die Routine LinearModelFit mit den Basisfunktionen $\left\{\sin 2 k \varphi, k=1, \ldots \frac{n}{2}-1\right\}$ für das trigonometrische Interpolationspolynom vom Grad $n$,

$$
\beta(\varphi) \approx \sum_{k=1}^{n / 2-1} b_{2 k} \sin 2 k \varphi
$$

- Die zu $\beta$ konjugierte Funktion ist bezüglich 0 und $\pi / 2$ gerade, sie lautet

$$
\mathcal{K}[\beta](\varphi) \approx \sum_{k=1}^{n / 2-1}-b_{2 k} \cos 2 k \varphi
$$

- An den Stützstellen $\varphi_{k}$ werden nun die zugehörigen Werte $\widetilde{r}\left(\varphi_{k}\right) \cos \beta\left(\varphi_{k}\right) e^{-\mathcal{K}[\beta]\left(\varphi_{k}\right)}$ berechnet. Man stellt fest, daß es sich hier wieder um eine bezüglich 0 und $\pi / 2$ gerade Funktion handelt:

$$
\widetilde{r} \cos \beta e^{-\mathcal{K}[\beta]}(\varphi) \approx \sum_{k=0}^{n / 2} \hat{a}_{2 k} \cos 2 k \varphi
$$

- Damit ergibt sich die ungerade Näherungsfunktion

$$
\mathcal{K}\left[\widetilde{r} \cos \beta e^{-\mathcal{K}[\beta]}\right](\varphi) \approx \sum_{k=1}^{n / 2-1} \hat{a}_{2 k} \sin 2 k \varphi
$$

- Durch NIntegrate läßt man sich bestätigen, daß $\widetilde{C}=0$ gilt

- $\psi_{i+1}(\varphi)$ nun direkt zu bilden würde nach sich ziehen, daß in jedem Iterationsschritt auf sämtliche Fourier-Entwicklungen der vorangegangenen Schleifen zurückgegriffen wird und das Verfahren sofort extrem langsam werden würde. Um dies zu umgehen ist es wesentlich, abschließend eine trigonometrische Interpolation der Werte $\psi_{i+1}\left(\varphi_{k}\right)$ durchführen zu lassen. Wie man leicht einsieht, sind die Funktionen $\psi_{i}(\varphi)$ bezüglich 0 und $\pi / 2$ stets ungerade Funktionen.
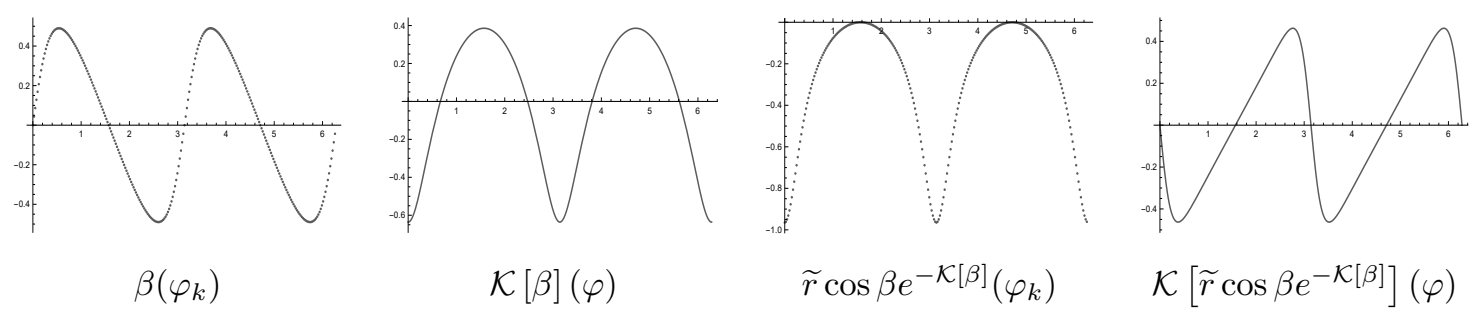

Abbildung 3.2: Durch Visualisierung der am NEwTonschen Verfahren beteiligten Funktionen gewinnt man Aufschluß darüber, wie die trigonometrischen Interpolationspolynome anzusetzen sind. 


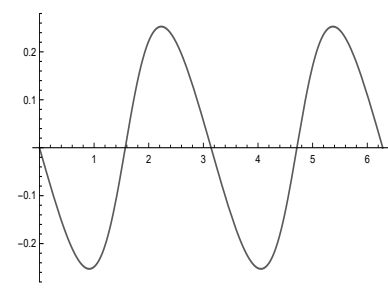

$e_{0}(\varphi)=\theta(\varphi)-\varphi$

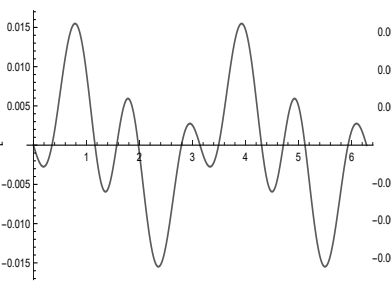

$e_{1}$

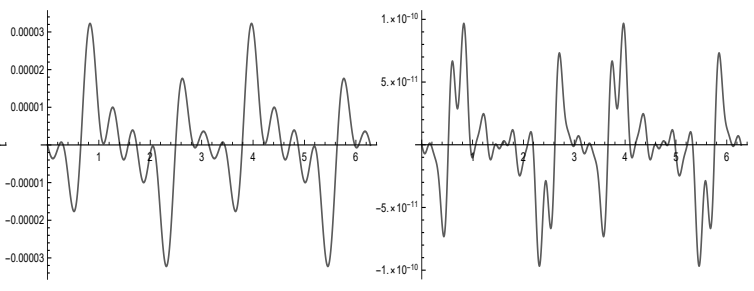

$e_{2}$

$e_{3}$

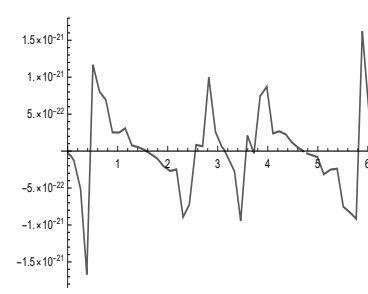

$e_{4}$

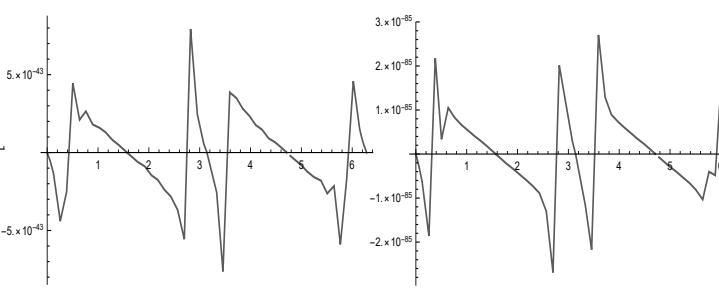

$e_{6}$

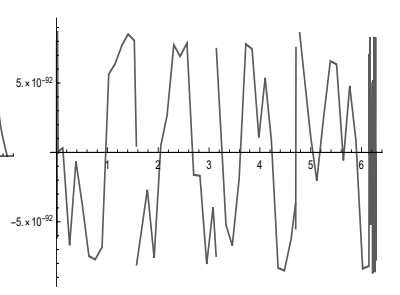

$e_{7}$

Abbildung 3.3: Die Konvergenz des NEwTonschen Verfahrens, implementiert nach HÜBNER, ist im Fall der gespiegelten Ellipse von bestechender Geschwindigkeit. Bei $2 n=1024$ am Einheitskreis verwendeten Stützstellen führt sie bereits nach sieben Iterationen bei einer Rechenzeit von etwa $15 \mathrm{~min}$ in die Gegend der vorgegebenen WorkingPrecision (100), der Fehler 3.1 liegt bei $5.10^{-92}$.

Damit ist die Ermittlung der Ränderzuordnungsfunktion gelöst. Die Verwendung von $2 n=256$ Stützstellen führt nach fünf Iterationsschritten auf einem Standard-Notebook ${ }^{3}$ in 20 s zu einem Fehler in der Größenordnung $2 \cdot 10^{-26}$, die ersten beiden Ableitungen sind auf $2 \cdot 10^{-24}$ bzw. $2 \cdot 10^{-22}$ genau. Mit Kenntnis der Ränderzuordnungsfunktion $\theta(\varphi)$ errechnen sich dann die Bilder der Peripherie des Einheitskreises aus $f(w)=\rho(\theta(\arg [w])) e^{i \theta(\arg [w])}$, zur Berechnung der inneren Punkte wurde die SchwARZsche Integralformel (2.17) herangezogen und die gesuchte konforme Abbildung vom Quadrat auf die gespiegelte Ellipse erhält man durch Komposition mit 2.7, vgl. Abbildung 3.4.
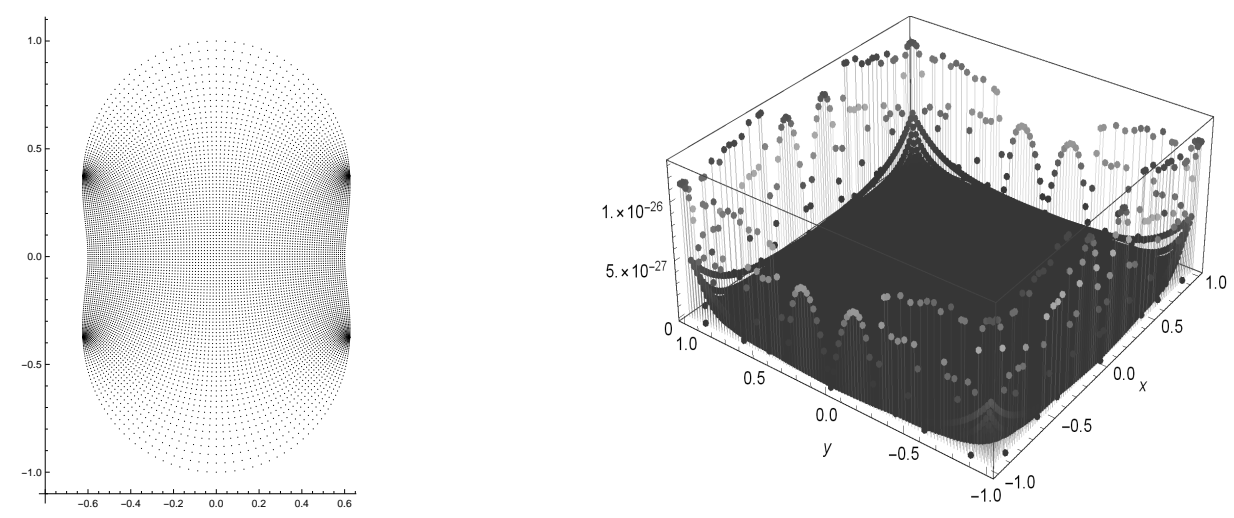

Abbildung 3.4: Konforme Abbildung von $\mathfrak{Q}_{101,101}$ über den Einheitskreis auf die gespiegelte Ellipse mit $p=0.6$ bei Verwendung von $N=2 n=256$ Stützstellen $\varphi_{k}$ (links) und Absolutbeträge der Fehler (rechts). Die Fehlermaxima werden am Rand angenommen.

\footnotetext{
${ }^{3}$ Toshiba Técra Z50-E / Intel Core i5-8250U / 8 GB DDR4-RAM 2400Mhz
} 


\subsection{Numerische Lösung der Quadratabbildung}

Der Rand des Einheitsquadrates kann auf $\left[-\frac{\pi}{4}, \frac{\pi}{4}\right]$ durch

$$
\rho(\theta)=\sec (\theta)
$$

parametrisiert werden, wobei wegen der Symmetrien sämtliche Informationen der konformen Abbildung vom Kreis auf das Einheitsquadrat aus dem Intervall $\left[0, \frac{\pi}{4}\right]$ gewonnen werden können. Die zum Vergleich mit der mit Mathematica errechneten Näherungslösung benötigte exakte konforme Abbildung sowie die Ränderzuordnungsfunktion lauten mit (2.6) bzw. [15, S.265]

$$
\zeta=f(w)=-\frac{2 \sqrt[4]{-1}}{\mathfrak{E}_{k ; \frac{1}{2}}} \mathfrak{E}_{f}[i \operatorname{arsinh}(\sqrt[4]{-1} w) ;-1] \quad \text { und } \quad \theta(\varphi)=\arctan \frac{\mathfrak{E}_{f}\left[\arcsin (\sqrt{2} \sin \varphi) ; \frac{1}{2}\right]}{\mathfrak{E}_{k ; \frac{1}{2}}} .
$$

Zur Behandlung der Theodorsenschen Integralgleichung bei Vorliegen eines Gebietes, dessen Berandung Ecken aufweist, ist zu erwarten, daß die Verwendung trigonometrischer Polynome zur Entwicklung von $\ln \rho(\theta(\varphi))$ für eine möglichst genaue Bestimmung der Ränderzuordnungsfunktion auf Grund der vorliegenden Sprungstellen von $\rho^{\prime}(\theta)$ keine geeignete Vorgehensweise darstellt. Hinsichtlich der in Abschnitt 2.2 erwähnten Untersuchung der Ableitung solcher Funktionen am Rand kann aber zunächst Folgendes festgestellt werden [3, S.22]:

Satz (Darstellung von Funktionen mit Ecken).

Wird das Gebiet $\mathfrak{G}$ von einer Kurve $\mathfrak{C}$ mit Ecken in $\zeta_{i}=f\left(w_{i}\right)$ und zugehörigen Innenwinkeln $\alpha_{i} \pi$ dergestalt berandet, daß deren Parametrisierung stückweise einer Lipschitzbedingung genügt, so gilt mit $g(w) \neq 0$ und stetig in $\overline{\mathfrak{E}}$

$$
f^{\prime}(w)=\prod_{i}\left(w-w_{i}\right)^{\alpha_{i}-1} g(w) .
$$

Man vergleiche dazu auch die in Abschnitt (12.2) in [23, S.327, Theorem (12.2.1)] angegebenen asymptotischen Entwicklungen. Für die Ableitung der Ränderzuordnungsfunktion in einem Eckpunkt $\zeta_{i}=f\left(e^{i \varphi_{i}}\right)$ gilt nach [3, S.38] die Darstellung

$$
\theta^{\prime}(\varphi)=\left|\varphi-\varphi_{i}\right|^{\alpha_{i}-1} \lambda(\varphi)
$$

und da es sich bei $\theta^{\prime}(\varphi)$ im gegenständlichen Fall um eine gerade Funktion handelt ergibt es Sinn, $\theta^{\prime}$ im Intervall $\left[-\frac{\pi}{4}, \frac{\pi}{4}\right]$ in der Form

$$
\theta^{\prime}(\varphi)=\left(\frac{\pi^{2}}{16}-\varphi^{2}\right)^{-\frac{1}{2}} \sum_{n=0}^{\infty} a_{2 n} \varphi^{2 n}
$$

anzusetzen. Auf die Gestalt von $\theta(\varphi)$ kann man dann durch partielle Integration schließen. Einen Code zur sukzessiven Approximation gibt RICHARDSON in [28, S.471ff.], wobei die Berechnung des Konjugiertenoperators durch direkte Auswertung des Integrals an nur 8 Stützstellen erfolgt und dann laut obigem mit Basisfunktionen

$$
\left\{\varphi^{2 n-1}\left(\frac{\pi^{2}}{16}-\varphi^{2}\right), \quad \varphi\left(\frac{\pi^{2}}{16}-\varphi^{2}\right)^{\frac{2 n-1}{2}}\right\}
$$

interpoliert wird. Der Fehler zur exakten Lösung bewegt sich dabei in der Größenordnung $10^{-7}$, das Verfahren beginnt nach etwa 50 Iterationsschritten zu stagnieren. Ausgehend von dieser Lösung ist es uns gelungen, unter Verwendung des von RICHARDSON angegebenen Programmes die Genauigkeit weiter zu verbessern, sodaß sich der Fehler nunmehr in der Gegend $10^{-11}$ bewegt. Dazu verwendet haben wir unter Hinzunahme der Stammfunktion von $1 / \sqrt{\pi^{2} / 16-\varphi^{2}}$ Basisfunktionen der Form

$$
\left\{\left(\frac{\pi^{2}}{16}-\varphi^{2}\right) \arcsin ^{2 n-1}\left(\frac{4 \varphi}{\pi}\right), \quad\left(\frac{\pi^{2}}{16}-\varphi^{2}\right)^{\frac{2 n-1}{2}} \arcsin \left(\frac{4 \varphi}{\pi}\right)\right\} .
$$




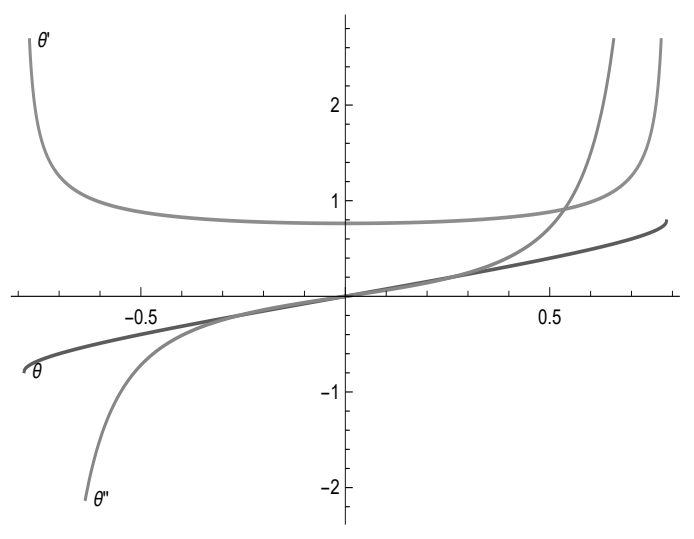

$\theta, \theta^{\prime}$ und $\theta^{\prime \prime}$

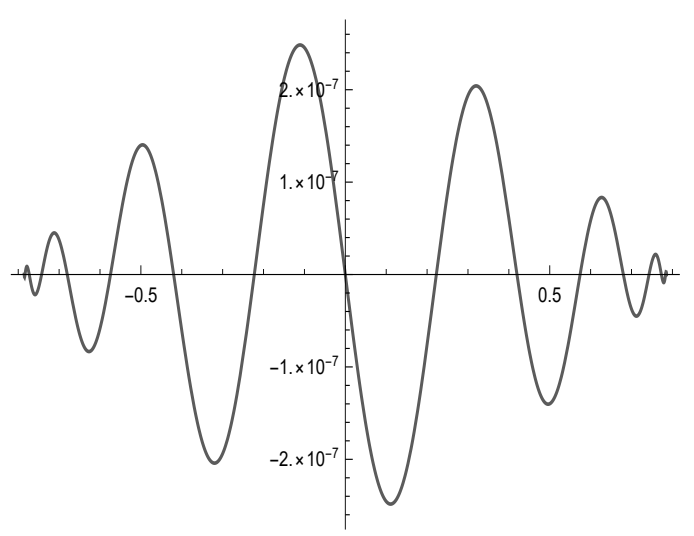

Fehler $\theta$ nach 60 Iterationen, RICHARDSON

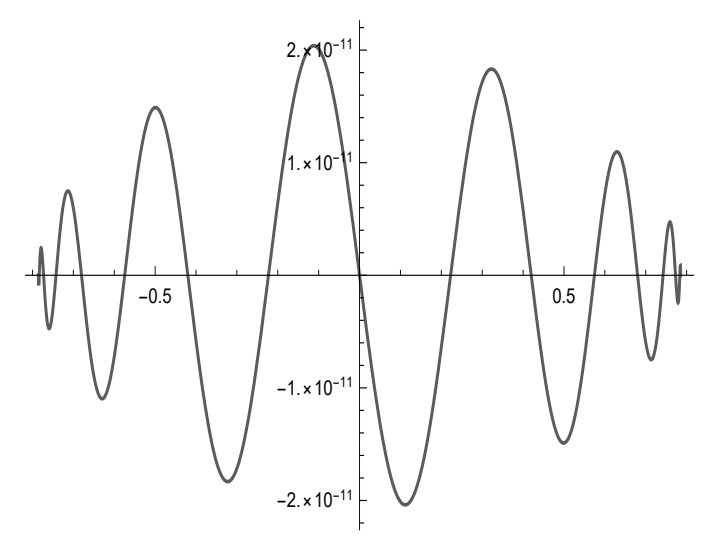

Fehler $\theta$ nach 40 Nachiterationen mit neuen Basisfunktionen

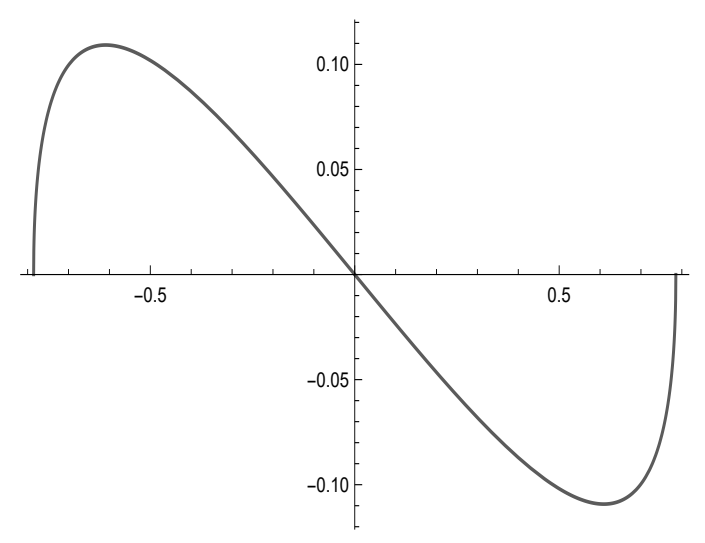

Der Startfehler $\theta(\varphi)-\varphi$

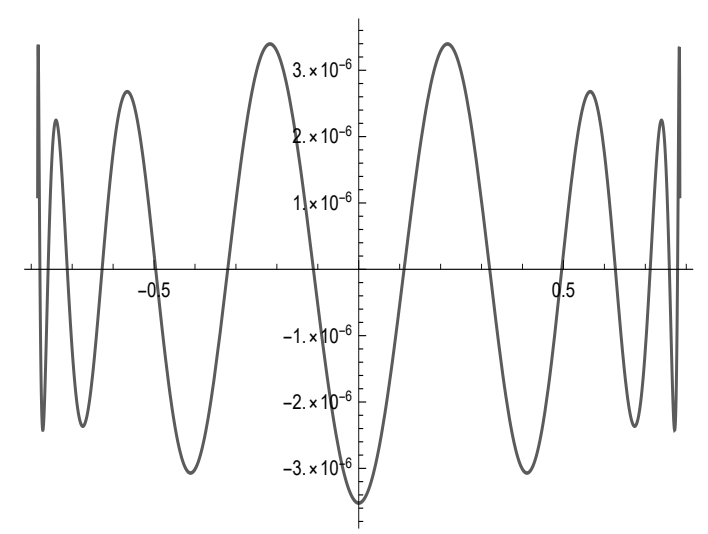

Fehler $\theta^{\prime}$ nach 60 Iterationen auf $\left[-\frac{\pi}{4}+10^{-4}, \frac{\pi}{4}-10^{-4}\right]$, RICHARDSON

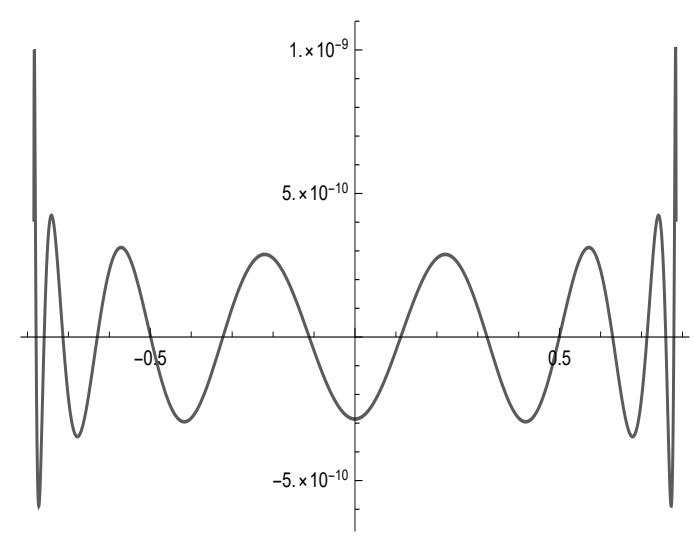

Fehler $\theta^{\prime}$ nach 40 Nachiterationen auf $\left[-\frac{\pi}{4}+10^{-4}, \frac{\pi}{4}-10^{-4}\right]$, neue Basisfunktionen

Abbildung 3.5: Untersuchung der Ränderzuordnung $\theta(\varphi)$ auf $\left[-\frac{\pi}{4}, \frac{\pi}{4}\right]$ zur Abbildung $\mathfrak{E} \rightarrow \mathfrak{Q}$. 
Wir stellen fest, daß auch in diesem Fall die Iterationen nach etwa 40 Schritten keine weiteren Verbesserungen mehr erbringen und daß einige mit dem Newtonverfahren durchgeführte Versuche auf Grund des Umstandes, daß die dort zu bildenden Hilfsfunktionen durchwegs singuläres Verhalten aufweisen, keine verwertbaren Ergebnisse liefern.

Die Näherung für $\theta$ ist auf dem gesamten Intervall $\left[-\frac{\pi}{4}, \frac{\pi}{4}\right]$ gültig. Was die Approximation der Ableitung $\theta^{\prime}(\varphi)$ betrifft, wird der Fehler bei einer Annäherung an die Intervallgrenzen $\pm \pi / 4$ bei RICHARDSON unbrauchbar groß, wobei er sich auf dem Intervall $\left[-\frac{\pi}{4}+10^{-4}, \frac{\pi}{4}-10^{-4}\right]$ in einer zufriedenstellenden Größenordnung von $10^{-6}$ bewegt. Dies ist insofern von Bedeutung, als $\theta^{\prime}$ bei einer späteren Simulation benötigt wird. Durch Verwendung der neuen Basisfunktionen kann diesbezüglich erreicht werden, daß sich der Fehler der Ableitung auch bei Annäherung an die Intervallgrenzen zufriedenstellend verhält: Im Intervall $\left[-\frac{\pi}{4}+10^{-9}, \frac{\pi}{4}-10^{-9}\right]$ bewegt er sich bei maximal $10^{-6}$.

Wir wollen in weiterer Folge das Rechengitter $\mathfrak{Q}_{101,101}$ über Vorschrift (2.7) zunächst geschlossen auf den Einheitskreis abbilden, die daraus resultierenden Gitterpunkte unter Zuhilfenahme der numerisch konstruierten Ränderzuordnungsfunktion sodann wieder zurück auf das Quadrat transformieren und uns in Abschnitt 4.6 nach dem dabei begangenen Simulationsfehler fragen.

\subsection{Abbildung auf ein rotationssymmetrisches Gebiet}

Im Falle einer realistischeren Problemstellung erwächst die Schwierigkeit, daß Ränderzuordnung und exakte konforme Abbildung nicht in geschlossener Form zur Verfügung stehen und somit (3.1) nicht zur Ermittlung des Fehlers herangezogen werden kann. Es können aber, bei kontinuierlicher Implementierung, die Differenzen $\theta_{i}(\varphi)-\theta_{i-1}(\varphi)$ betrachtet werden, um Aufschluß über die Konvergenz der Iterationen zu erhalten. Analog dazu kann man mit dem $i$-ten Iterationsvektor $\theta_{i}^{(k)}:=\theta_{i}\left(\varphi_{k}\right), \quad \varphi_{k}=\frac{k \pi}{n}$, komponentenweise den diskreten Fehlervektor $e_{i}^{(k)}:=\theta_{i}^{(k)}-\theta_{i-1}^{(k)}$ bilden.

Mit $N=2 n$ werde nachfolgend stets eine gerade Anzahl an äquidistanten Stützstellen am Einheitskreis vereinbart, bei Verwendung der diskreten Fourier-Transformation ist zusätzlich zu beachten, daß die FFT-Algorithmen sehr effizient mit $N=2^{2 n}$ Stützstellen arbeiten.

\subsubsection{Definition der Geometrien}

Hinsichtlich der im abschließenden Kapitel durchzuführenden Simulation eines Zahnmodells soll ein sternförmiges Gebiet zu Grunde gelegt werden, das dem Querschnitt eines Zahnes nahekommt. Da durch Rotation um die $\eta$-Achse ein dreidimensionales Modell erzeugt werden kann ist es hinreichend, die Betrachtungen auf das abgeschlossene Intervall $\left[\frac{\pi}{2}, \frac{3 \pi}{2}\right]$ zu beschränken, dabei die auf $[0,2 \pi)$ vorhandenen Symmetrien zu untersuchen und bei der Implementierung zu nutzen. So kann bereits bei der Geometriebeschreibung durch trigonometrische Interpolation davon ausgegangen werden, daß die auftretenden Cosinus-Terme sämtlich gerade und die Sinus-Terme alle ungerade sind.

Wegen der wünschenswerten Glattheitseigenschaften von $\rho(\theta)$ bietet sich die Vorgabe von Randpunkten mit anschließender Fourier-Interpolation ${ }^{4}$ zur Beschreibung der Berandung an. Es werden dahingehend zwei Alternativen in Abhängigkeit der $\varepsilon$-Bedingung betrachtet, eine dritte Definition soll die Möglichkeiten der periodischen Hermite-Interpolation ${ }^{5}$ aufzeigen. Neben den Punktkoordinaten in Polardarstellung können bei dieser Art der Interpolation optional auch Ableitungen vorgeschrieben werden, allerdings ist die Angabe einer WorkingPrecision in diesem Fall nicht mehr möglich und die Genauigkeit wird auf \$MachinePrecision beschränkt. Tabelle 3.1 listet die verwendeten Werte, Abbildung 3.6 visualisiert die Gebiete und die zugehörigen $\varepsilon$-Bedingungen.

\footnotetext{
${ }^{4}$ Bei 16 äquidistanten Stützstellen implementiert man das trigonometrische Polynom vom Grad 8 durch basis=Union [Table [Cos [2m*phi], \{m, 0,4\}], Table [Sin [(2m-1)*phi] , \{m, 1, 4\}]];

$\mathrm{lm}=$ LinearModelFit [geo, basis, phi, WorkingPrecision $\rightarrow$ nprec, IncludeConstantBasis $\rightarrow$ False] ;

$r$ [phi_]=SetPrecision [lm ["BestFit", WorkingPrecision $\rightarrow$ nprec], Infinity] ;

${ }^{5}$ rfloat=Interpolation [geo, InterpolationOrder $\rightarrow 3$, PeriodicInterpolation $\rightarrow$ True, Method $\rightarrow$ "Hermite"] ;

$r$ [phi_]=SetPrecision [rfloat [phi], Infinity];
} 


\begin{tabular}{|c||c||c||c|c|c|}
\multicolumn{1}{c}{ GEO1 } & \multicolumn{1}{c}{ GEO2 } & \multicolumn{2}{c}{ GEO3 (Hermite) } \\
\hline \hline$\theta \in[0,2 \pi]$ & $\rho_{1}(\theta)$ & $\rho_{2}(\theta)$ & $\rho_{3}(\theta)$ & $\rho_{3}^{\prime}(\theta)$ & $\rho_{3}^{\prime \prime}(\theta)$ \\
\hline \hline 0 & 7 & 6 & 5 & 5 & 20 \\
\hline$\pi / 8$ & 9 & 9 & - & - & - \\
\hline $2 \pi / 8$ & 9 & $7 \sqrt{2}$ & $7 \sqrt{2}$ & 0 & -30 \\
\hline $3 \pi / 8$ & $15 / 2$ & $15 / 2$ & - & - & - \\
\hline $4 \pi / 8$ & $13 / 2$ & 7 & 7 & 0 & 25 \\
\hline $5 \pi / 8$ & $15 / 2$ & $15 / 2$ & - & - & - \\
\hline $6 \pi / 8$ & 9 & $7 \sqrt{2}$ & $7 \sqrt{2}$ & 0 & -30 \\
\hline $7 \pi / 8$ & 9 & 9 & - & - & - \\
\hline$\pi$ & 7 & 6 & 5 & -5 & 20 \\
\hline $9 \pi / 8$ & $11 / 2$ & 5 & - & - & - \\
\hline $10 \pi / 8$ & 6 & 5 & $7 \sqrt{2} / 2$ & 3 & 5 \\
\hline $11 \pi / 8$ & 8 & $15 / 2$ & $10 \sqrt{2} / 2$ & 10 & 30 \\
\hline $12 \pi / 8$ & 10 & 10 & 10 & 0 & -50 \\
\hline $13 \pi / 8$ & 8 & $15 / 2$ & $10 \sqrt{2} / 2$ & -10 & 30 \\
\hline $14 \pi / 8$ & 6 & 5 & $7 \sqrt{2} / 2$ & -3 & 5 \\
\hline $15 \pi / 8$ & $11 / 2$ & 5 & - & - & - \\
\hline $2 \pi$ & - & - & 5 & 5 & 20 \\
\hline
\end{tabular}

Tabelle 3.1: Randdefinitionen durch Vorgabe von Punkten und Ableitungen.

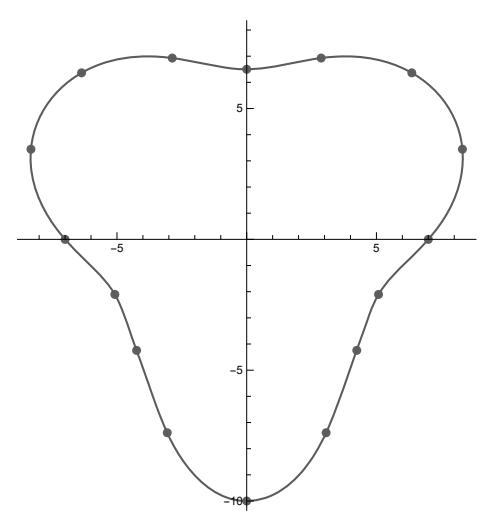

Geometrie 1 (Fourier)

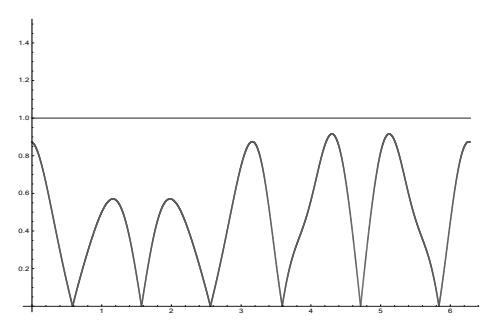

$|\sigma(\theta)|<1$

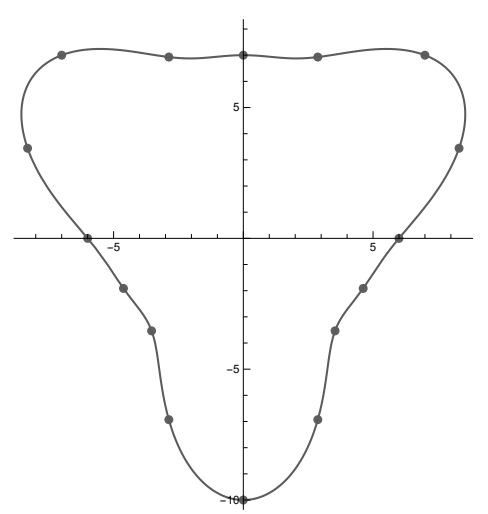

Geometrie 2 (Fourier)

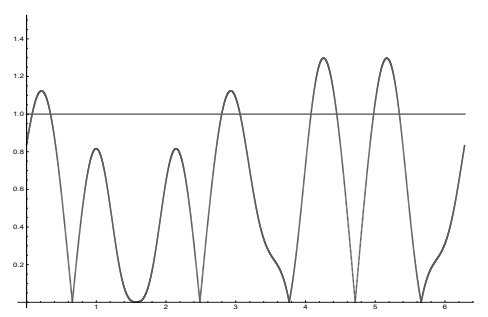

teilweise $|\sigma(\theta)|>1$

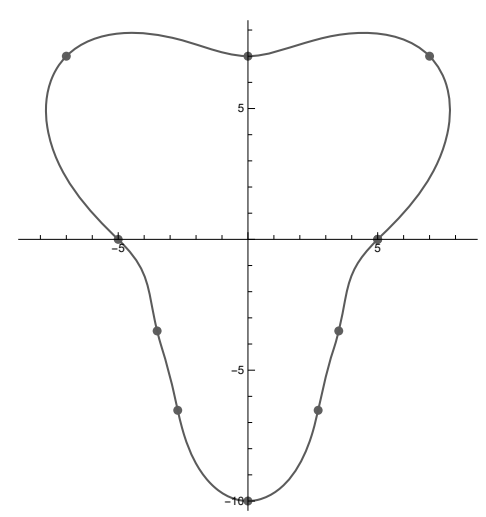

Geometrie 3 (Hermite)

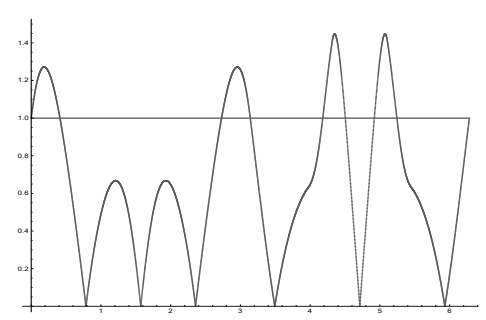

teilweise $|\sigma(\theta)|>1$

Abbildung 3.6: Punkte und Interpolierende der Randkurven (oben). Mit symmetrischem $\rho(\theta)$ wird $\sigma(\theta)$ in den Intervallen $[0, \pi]$ bzw. $[\pi, 2 \pi]$ schiefsymmetrisch bezüglich $\pi / 2$ bzw. $3 \pi / 2$ (unten). 


\subsubsection{Die Newtonmethode von HÜBNER nach WEGMANN}

Wie die bei der Iteration der gespiegelten Ellipse gewonnenen Erfahrungen lehren, konvergiert das Newtonverfahren extrem schnell und liefert eine hochgenaue Ränderzuordnungsfunktion. Leider lassen sich diese Aussagen nicht ohne weiteres auf allgemeinere Gebiete übertragen, bei denen die quadratische Konvergenz häufig im Zuge der Berechnung von einem Iterationsschritt zum nächsten ohne ersichtlichen Grund verloren geht und im besten Falle linear und träge wird. Die Ursache dafür ist in der Approximationsgüte der trigonometrischen Interpolation zu finden, die involvierten Hilfsfunktionen werden in gewissen Punkten zunehmend spitzer (wobei aber deren Analytizität dort erhalten bleibt) oder weisen lokal immer größere Schwankungen auf, ein für ein trigonometrisches Interpolationspolynom wenig erfreulicher Umstand. Zusätzlich bedeutet der Übergang zu allgemeineren sternförmigen Gebieten den Verlust von Symmetrien, was sich in der Erhöhung der Anzahl nichtverschwindender Fourierkoeffizienten manifestiert und einerseits mehr Rechenaufwand, andererseits eine größere numerische Fehlerquelle mit sich bringt.

Zielführend zur weiteren Steigerung der Genauigkeit und Senkung der Rechenzeiten ist ein Blick auf die im Zuge der Vorstellung des Newtonverfahrens bereits erwähnte Arbeit von WEGMANN. Er gibt, ausgehend von einem Startvektor $\boldsymbol{\theta}_{0}=\theta_{0}^{(k)}$, in [34, S.218, Abschn.6] dazu die folgende diskrete Iterationsvorschrift ${ }^{6}$ :

$$
\boldsymbol{\theta}_{i+1}=\boldsymbol{\theta}_{i}-\frac{\mathbf{f}}{\mathbf{r}^{2}}-\frac{(\mathbf{p}+\hat{q} \cdot \tan \hat{v}) \cdot \exp (\mathbf{w})}{\mathbf{r}}
$$

Um die darin enthaltene Symbolik zu erörtern (vektorielle Größen sind dabei in Fettdruck gesetzt und deren Operationen sind komponentenweise zu verstehen), wird zunächst der KonjugiertenOperator $\mathcal{K}$ in seiner diskreten Form eingeführt, der dann als WiTTICH-Operator bezeichnete diskrete Operator $\mathcal{K}_{N}$ transformiert eine vektorielle Größe $\mathbf{h}$ vermöge ihrer diskreten Fourier-Koeffizienten nach der Vorschrift

$$
\mathcal{K}_{N}[\mathbf{h}]=\sum_{j=1}^{n-1} a_{j} \sin j \varphi-b_{j} \cos j \varphi
$$

wobei

$$
a_{j}=\frac{1}{n} \sum_{k=1}^{N} \mathbf{h}^{(k)} \cos j \boldsymbol{\varphi}^{(k)}, \quad b_{j}=\frac{1}{n} \sum_{k=1}^{N} \mathbf{h}^{(k)} \sin j \varphi^{(k)} .
$$

Dabei ist speziell zu beachten, daß neben $b_{0}$ und $b_{n}$ auch die Koeffizienten $a_{0}$ und $a_{n}$ nicht in die Summation einfließen. Neu ist dies aber lediglich für $a_{n}$, dieser Koeffizient wird bei der Bildung von $\hat{q}$ bzw. $\hat{v}$ zur Hälfte seine Berücksichtigung finden. Mit diesen Vorbetrachtungen sind im $i+1$-ten Iterationsschritt die folgenden Hilfsgrößen zu berechnen:

$$
\mathbf{f}=\boldsymbol{\theta}_{i}-\boldsymbol{\varphi}-\mathcal{K}_{N}\left[\ln \rho\left(\boldsymbol{\theta}_{i}\right)\right], \quad \mathbf{r}=\sqrt{1+\sigma^{2}\left(\boldsymbol{\theta}_{i}\right)}, \quad \mathbf{v}=\arctan \sigma\left(\boldsymbol{\theta}_{i}\right), \quad \mathbf{w}=\mathcal{K}_{N}[\mathbf{v}],
$$

und weiters

$$
\hat{v}=\frac{1}{N} \sum_{k=1}^{N} \mathbf{v}^{(k)}, \quad \mathbf{q}=\frac{\mathbf{f} \cdot \sigma\left(\boldsymbol{\theta}_{i}\right)}{\mathbf{r} \cdot \exp (\mathbf{w})}, \quad \mathbf{p}=\mathcal{K}_{N}[\mathbf{q}], \quad \hat{q}=\frac{1}{N} \sum_{k=1}^{N} \mathbf{q}^{(k)}
$$

Mit dem Verfahren von WEgmann lassen sich auf den in diesem Abschnitt betrachteten Gebieten die besten Ergebnisse erzielen. Wenn auch die Problematik hinsichtlich der Fourier-Approximation damit nicht beseitigt wird, so wird man auf Grund des Ressourcen sparenden Algorithmus doch in die Lage versetzt, die Anzahl der Stützstellen noch etwas weiter zu steigern und damit die Auflösung im Bereich der Problemstellen weiter zu erhöhen. Natürlich sind aber auch dieser Vorgehensweise, nicht zuletzt wegen der als äquidistant vorauszusetzenden Stützstellen $\varphi_{k}$, Grenzen gesetzt.

\footnotetext{
${ }^{6}$ Während HüBner die Differenz $\psi=\theta-\varphi$ iteriert, formuliert WEgmann sein Verfahren direkt für $\theta$.
} 


\subsubsection{Bemerkungen zur Implementierung}

Die Verwendung einer großen Anzahl an Stützstellen bedingt eine sorgfältige Untersuchung der beteiligten Datenstrukturen, um den verfügbaren Speicher und die Prozessorleistung möglichst umsichtig einzusetzen. Die folgenden Überlegungen sind zur Optimierung des Codes dabei hilfreich.

Symbolisches versus numerisches Rechnen. Generell ist festzustellen, daß durch symbolisches Rechnen die Kalkulationen eher verlangsamt werden. Schon bei der Radiusfunktion steht man vor der Entscheidung, in welcher Weise deren Werte bereitgestellt werden sollen. Während bei der gespiegelten Ellipse und beim Quadrat die Berandung in geschlossener Form vorliegt und somit symbolisch behandelt werden kann, bietet sich wegen der Interpolation des Randes der rotationssymmetrischen Gebiete eher der numerische Ansatz an. Mit SetPrecision[r[phi_],p] wird die Genauigkeit vorgegeben, wobei aus der Setzung $\mathrm{p}=$ Infinity eine rationale Darstellung resultiert. Durch \$MinPrecision=n kann die untere Schranke der global geforderten Präzision der Nachkommastellen vorgeschrieben werden — diese ist unter Beachtung der gewünschten Resultatsgenauigkeit nicht unnötig hoch vorzugeben, andernfalls machen sich schnell Engpässe im Arbeitsspeicher bemerkbar. Die Werte für die äquidistanten $\varphi_{k}$ am Einheitskreis werden ebenfalls in Gleitkommadarstellung initialisiert, bei symbolischer Definition zeigt sich nämlich, daß bei Verwendung interner Routinen durchaus die Möglichkeit besteht, daß die Genauigkeit auf \$MachinePrecision zurückfällt. $\mathrm{Zu}$ Testzwecken empfiehlt sich, die Anzahl der signifikanten Nachkommastellen der Rechengrößen durch den Befehl Precision[expr] zu kontrollieren.

Verwendung der diskreten Fourier-Transformation. Testläufe legen die Verwendung der Routine Fourier [] zur Berechnung der Fourier-Koeffizienten mit $N=2^{2 n}$ Stützstellen am Einheitskreis nahe. Sie liefert zu einem vorgegebenen Datensatz $\left(\theta_{k}\right), k=1, \ldots N$, einen gleichdimensionalen Satz (komplexer) Werte $\left(c_{k}\right)$, mit Hilfe derer ein (reelles) Näherungspolynom $P_{m}\left(\varphi, c_{k}\right)$ konstruiert werden kann, das im Falle $m<N$ eine Kleinstquadrate-Approximation und für $m=N$ die Interpolierende darstellt ${ }^{7}$. Es lautet für $\varphi_{k} \in[0,2 \pi)$ und einer geraden Anzahl von Stützstellen

$$
P_{m}\left(\varphi, c_{k}\right)=\frac{\Re\left(c_{1}\right)+\Re\left(c_{1+\frac{N}{2}}\right) \cos \frac{m}{2} \varphi}{\sqrt{N}}+\frac{2}{\sqrt{N}} \sum_{k=1}^{\frac{m}{2}-1} \Re\left(c_{k+1} \cos k \varphi\right)-\Im\left(c_{k+1} \sin k \varphi\right) .
$$

An die reellen Fourier-Koeffizienten gelangt man durch Anwendung des Befehles Expand [] auf $P_{N}$. Wieder ist es ratsam, die Ergebnisse der Implementierung durch Vergleich mit LinearModelFit [] abzugleichen. In beiden Fällen wird die Genauigkeit der Rechnung hinsichtlich der Nachkommastellen der Koeffizienten nicht durch \$MachinePrecision beschränkt ${ }^{8}$. Testweise sollten die FourierKoeffizienten auch über die bekannten Formeln ermittelt und mit der von Mathematica durchgeführten diskreten Fourier-Transformation verglichen werden, um Implementierungsfehler auszuschließen.

Identifizierung mehrfach verwendeter Größen. Daß innerhalb eines Iterationsschrittes mehrfach verwendete Größen nur einmalig evaluiert und dann gesondert abgespeichert werden sollten ist eine Offensichtlichkeit, dies betrifft vor allem den Vektor $\sigma\left(\boldsymbol{\theta}_{i}\right)$. Zu einer extremen Verkürzung der Rechenzeiten eines Iterationsschrittes gelangt man, wenn man erkennt, daß Größen wie $\sin \left(2 m \varphi_{k}\right)$, die an der dreimaligen Auswertung des Wittich-Operators beteiligt sind, als konstante $\frac{N}{4} \times N$-Matrizen vorab außerhalb der eigentlichen Iterationen berechnet werden können oder noch besser extern abgespeichert und eingelesen werden. Der Gewinn an Rechenzeit zieht allerdings einen in Abhängigkeit der Stützstellen mitunter drastisch hohen Bedarf an Arbeitsspeicher nach sich. Unter Berücksichtigung des nächsten Optimierungspunktes wird zusätzlich noch die Senkung der Matrixdimension auf $\frac{N}{4} \times\left(\frac{N}{2}+1\right)$ möglich.

\footnotetext{
${ }^{7}$ Vgl. http://demonstrations.wolfram.com/TrigonometricFittingAndInterpolation

Für das reelle Interpolationspolynom und dessen Koeffizienten siehe [7, S.456f., (18.34)-(18.36)]

${ }^{8}$ Die Anzahl $\mathrm{n}$ der Nachkommastellen der Fourier-Koeffizienten erzwingt man mit

Block [ $\{\$$ MinPrecision=n, $\$$ MaxPrecision=n $\}, f k l n R=$ Fourier [fvalues, FourierParameters $\rightarrow\{0,-1\}]]$;

lnRfourier[phi_] = Expand [poly[phi,Dimensions [fklnR] [[1]],fklnR]];

anlnR = Append [Coefficient [lnRfourier [phi], Table [Cos [2*m*phi], \{m,1,npunkte/2-1\}]], 0];

bnlnR = Coefficient [lnRfourier [phi], Table [Sin [(2*m-1)*phi], \{m,1,npunkte/2\}]];
} 
Einprogrammierung der Symmetrien. Es ist auf Grund der vorhandenen Symmetrien prinzipiell möglich, die vektoriellen Größen nur auf dem Intervall $[\pi / 2,3 \pi / 2]$ anzulegen und auszuwerten, die Ausdehnung auf $[0,2 \pi]$ kann erforderlichenfalls durch Einzeiler bewerkstelligt werden ${ }^{9}$. Dies birgt vor allem großes Einsparungspotential bei Bildung des Wittich-Operators.

Berechnung der Werte von $f(z)$ und $f^{(k)}(z)$ auf $\mathfrak{Q}_{n}$. Einer der großen Vorteile bei Verwendung der trigonometrischen Interpolation von $\ln \rho(\theta(\varphi))$ im Zuge des Verfahrens ist der Umstand, daß bei Berechnung der Fourier-Koeffizienten zur anschließenden Bildung des Wittich-Operators diese auch zur späteren Konstruktion von $f$ und deren Ableitungen im Inneren des Einheitskreises (und auch auf dessen Peripherie) herangezogen werden können. Man wertet dazu sehr einfach das diskrete Analogon von (2.16) aus, das sich sofort auch gliedweise differenzieren läßt. Dieser Umstand ist nicht zu unterschätzen, da der Weg über die Integralformeln einerseits mit hohem Rechenaufwand verbunden ist, andererseits macht die Berechnung der Ableitungen am Rand eine gesonderte theoretische Untersuchung hinsichtlich der Randwerte der Ableitungen der auftretenden Cauchy-Integrale erforderlich. Für die Ableitungen der Abbildung $\overline{\mathfrak{Q}} \rightarrow \overline{\mathfrak{G}}$ ist die Kettenregel anzuwenden.

Mit diesen Optimierungen löst der Algorithmus von WEGMANn die Ränderzuordnung der gespiegelten Ellipse bei gleichbleibender Genauigkeit um den Faktor 60 schneller (in etwa $15 s$ statt 15min).

\subsubsection{Ergebnisse}

Mit den in den vorangegangenen Abschnitten vorgestellten Verfahren kann nun die Ränderzuordnungsfunktion iteriert werden. Für Geometrie 1 liefert die Methode der sukzessiven Approximation auf Grund der erfüllten $\varepsilon$-Bedingung brauchbare Ergebnisse, wohingegen es — wie die Theorie vorhersagt - für die Gebiete 2 und 3 nicht konvergiert. Das Newtonverfahren nach HüBNER kann für alle Gebiete erfolgreich verwendet werden, allerdings verlangt die Gestalt der Hilfsfunktionen auf den beiden nicht kreisnahen Gebieten zur Auflösung der zunehmenden Schwankungen die Erhöhung der Stützstellen, was sich vor allem in der kontinuierlichen Fassung sehr empfindlich auf die Rechenzeiten auswirkt, namentlich wenn der Konjugiertenoperator durch NIntegrate errechnet wird. Die besten Resultate hinsichtlich Rechenzeit und Genauigkeit werden mit der diskreten WEGMANN-Iteration erzielt, nur sie sollen daher für die drei Gebiete präsentiert werden und nur die zugehörigen Ränderzuordnungsfunktionen sollen Eingang in die weiteren Untersuchungen finden.

Die letzten im Zuge der Iteration berechneten Fourier-Koeffizienten von $\ln \rho(\theta(\varphi))$ werden zur späteren Verwendung extern abgespeichert, damit sie zur Konstruktion der Abbildung für verschiedene Rechengitter jederzeit zur Verfügung stehen. Da die Ermittlung der Koeffizienten und der Ränderzuordnung je Geometrie nur einmalig erfolgt, lohnt die Verwendung einer sehr hohen Anzahl an Nachkommastellen (500, kann dann nach Bedarf reduziert werden) und einer großen Anzahl an Iterationsschritten (500). Die Rechenzeiten bei 1024 Stützstellen auf dem Standard-Notebook (vgl. Fußnote 3 auf Seite 34) betragen jeweils etwa 20min. Die vielen Iterationsschritte sind auch deshalb notwendig, weil die Konvergenz nach sieben Iterationen in den linearen Bereich zurückfällt.

In allen drei Fällen ist bei der Ränderzuordnungsfunktion eine Translation des Intervalls $[0,2 \pi]$ festzustellen - ein Sachverhalt, der jedoch unerheblich für die konforme Abbildung an sich ist. Ferner bewirkt eine zunehmende Verletzung der Kreisnähe die Verlangsamung der Konvergenz, was aber erforderlichenfalls durch eine Erhöhung der Anzahl an Iterationsschritten abgefangen werden kann. Abbildung 3.7 zeigt für die drei Geometrien die Abbildung des Rechengitters, die zugehörigen Ränderzuordnungsfunktionen sowie den diskreten Fehler.

Abschließend kann man sich noch die Frage nach der Qualität der konformen Abbildung stellen. Auf Grund der involvierten Funktionen der Bauart $w \exp [P(w)]$ darf erwartet werden, daß die Güte der Approximation dabei im Inneren höher als auf der Berandung der Gebiete ist. Wird mit $R(\theta)$ die interpolierende Funktion bezeichnet, die zur Beschreibung der Geometrie herangezogen wird (Fourier- oder Hermite-Interpolation), so kann der Ausdruck $R\left(\arg f\left(e^{i \varphi}\right)\right)-\left|f\left(e^{i \varphi}\right)\right|$ einen

\footnotetext{
${ }^{9} \mathrm{Zu}$ unterscheiden je nach Art der Symmetrie, für den schiefsymmetrischen Fall durch

oddextension [v_] :=Flatten [Append [Prepend [v,Table [-v [ [N/4+1-k]] , $\{\mathrm{k}, 0, \mathrm{~N} / 4-1\}]]$, Table $[-\mathrm{v}[[\mathrm{N} / 4-\mathrm{k}]],\{\mathrm{k}, 0, \mathrm{~N} / 4-2\}]]]$
} 

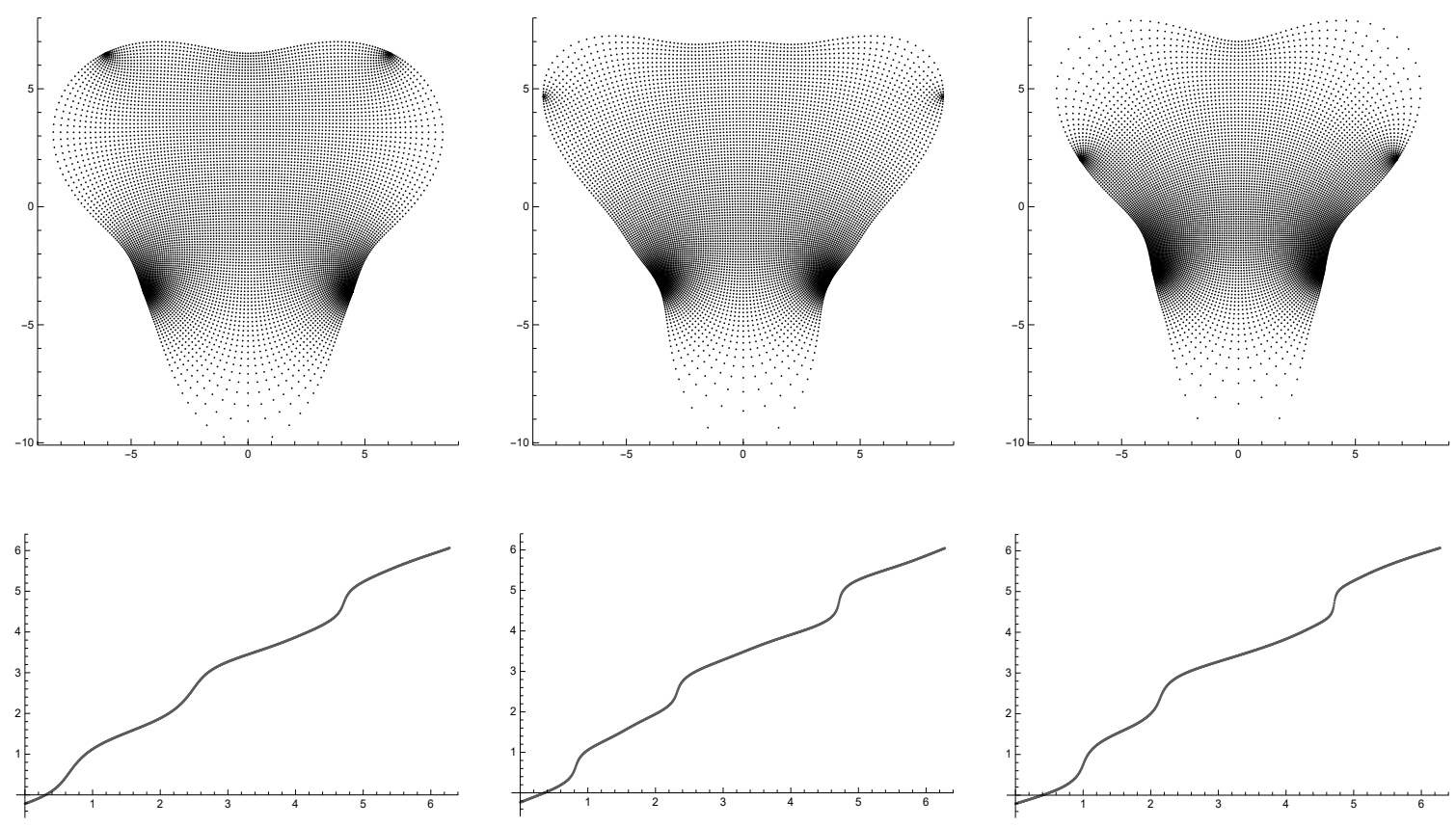

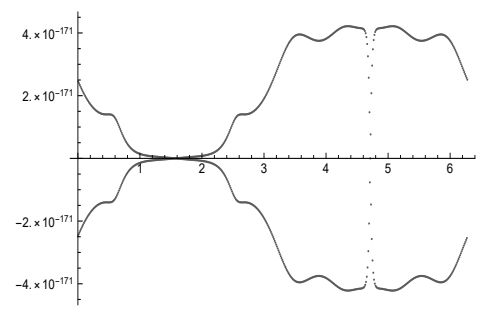

Geometrie 1 (Fourier)

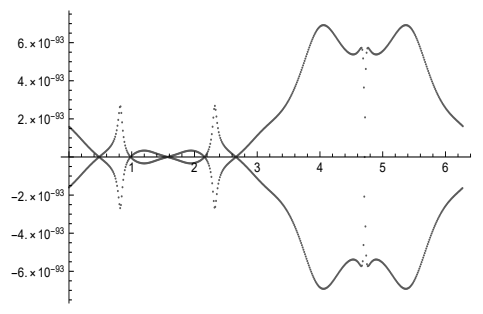

Geometrie 2 (Fourier)

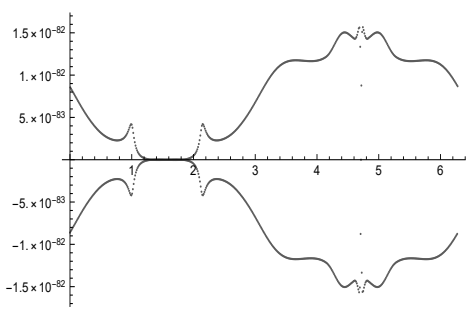

Geometrie 3 (Hermite)

Abbildung 3.7: Ergebnisse der konformen Abbildung $\mathfrak{Q}_{101,101} \rightarrow \mathfrak{G}$. Bemerkenswert ist, wie sich eine scheinbar geringe Abänderung der Geometrie auf die Lage der Bilder der Quadratecken auswirkt (oben). Zugehörige Ränderzuordnungsfunktionen (Mitte) und diskreter Fehlervektor $e_{500}^{(k)}$ (unten).

Anhaltspunkt über die Verhältnisse des Fehlers am Rand geben. Nach Konstruktion erbringt die Auswertung an den N Stützstellen $\varphi=\varphi_{k}$ erwartungsgemäß ein hochgenaues Ergebnis (bis in den Bereich der vorgegebenen Anzahl an Nachkommastellen), läßt man den Fehler jedoch im gesamten Intervall $[0,2 \pi]$ grafisch ausgeben (vgl. Abbildung 3.8 so wird deutlich, daß sich zwischen den Stützstellen die Verhältnisse — wieder in Abhängigkeit der Kreisnähe — deutlich verschlechtern.

Diese Einsicht ist von großer Bedeutung, da in diese Fehlerbetrachtungen nur die konforme Abbildung vom Einheitskreis auf das Gebiet einfließen. Konkret wäre man demnach in der Lage, durch Verwendung der Werte $\varphi_{k}$ als Grundlage der Rechengitterpunkte die Genauigkeit nach Belieben zu steigern. Die Verhältnisse sind aber derart gelagert, daß, vom Quadrat kommend, der Einheitskreis der Abbildung auf das Gebiet zwischengeschaltet wird. Da die Randgitterpunkte des Quadrates aber im allgemeinen nicht auf die Stützstellen $\varphi_{k}$ abgebildet werden, hat man sich an einem Fehler zu orientieren, der sich (bei 1024 Stützstellen) geometrieabhängig zwischen $10^{-4}$ und $10^{-11}$ bewegt. Weiters ist zu erwarten, daß sich die Fehlerverhältnisse bei Betrachtung der Ableitung der konformen Abbildung, namentlich an der Berandung, nicht wesentlich verbessern werden. Diese Erkenntnisse führen uns zu dem Schluß, daß für eine weitere Senkung des globalen Fehlers erforderlichenfalls die Anzahl der Stützstellen weiter erhöht werden muß. 

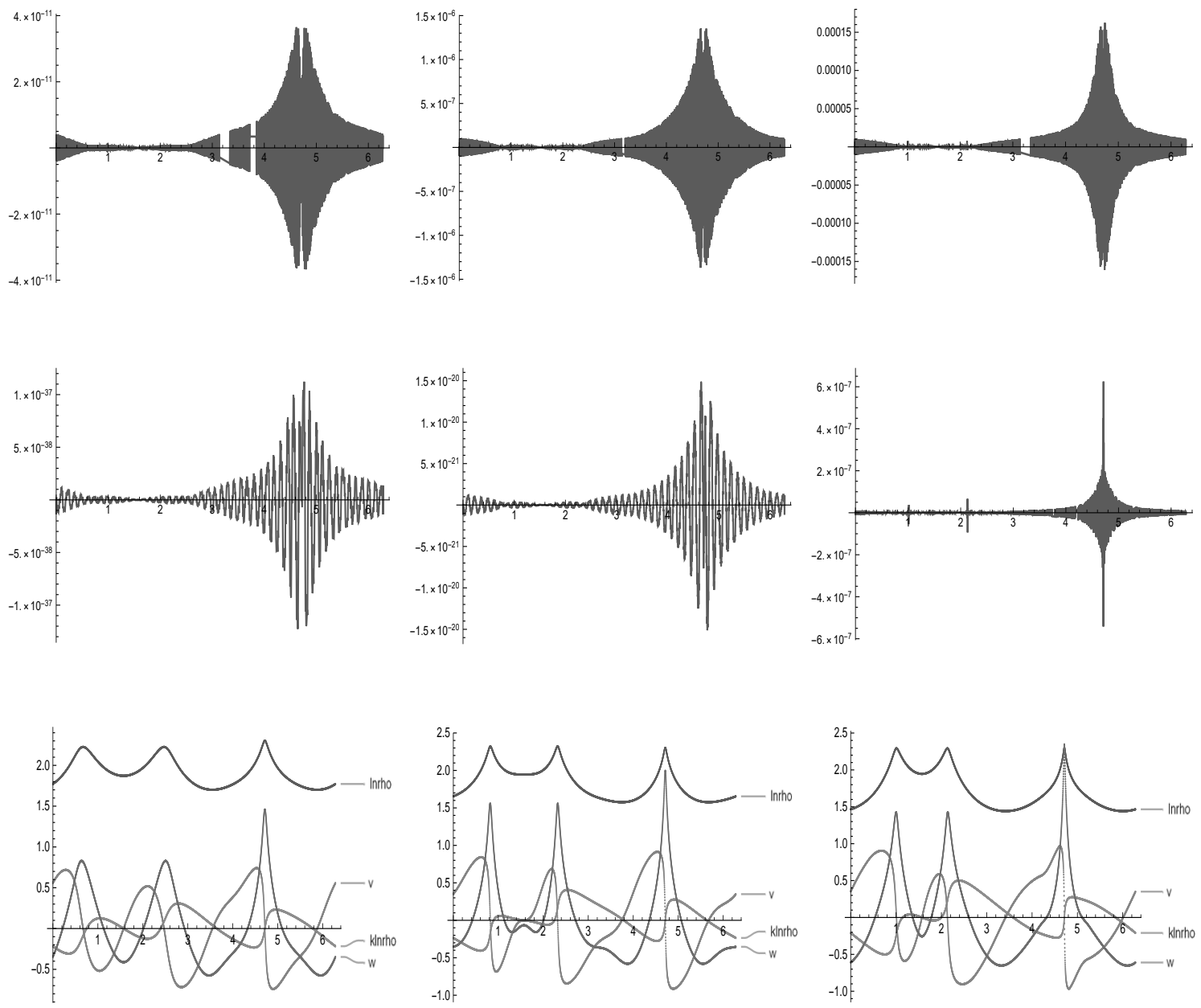

Geometrie 1 (Fourier)

Geometrie 2 (Fourier)

Geometrie 3 (Hermite)

Abbildung 3.8: Der globale Fehler bei Verwendung von 1024 (oben) und 4096 Stützstellen (Mitte). Im Zuge der Iteration verwendete Hilfsvektoren (unten).

Wegen der Qualität des Verfahrens von WEGMANN soll der der Implementierung zu Grunde liegende Quellcode abschließend auch noch explizit angeführt werden. Von primärem Interesse sind für die spätere Berechnung der Metrik die Fourier-Koeffizienten von $\ln \rho(\theta(\varphi))$, sie werden daher nach Abschluß der Iterationen in der Form

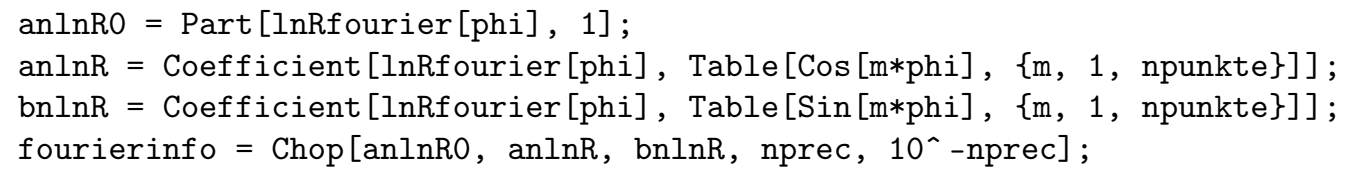

mittels des Befehls DumpSave extern in eine Datei abgespeichert, sodaß sie zur weiteren Verwendung ohne Neuberechnung eingelesen werden können und jederzeit zur Verfügung stehen. 
(* Implementierung der diskreten Newton-Iteration nach Hübner/Wegmann *) Clear ["Global`*"]; WD=FileNameTake [NotebookDirectory [] ,5] ; SetDirectory [WD] ; nprec=nprecfourier $=500 ; \mathrm{znp}=4096 ; \mathrm{np}=\mathrm{znp} / 2 ; \mathrm{niter}=500$;

(* Geometrie laden - zurück kommt verwendbares r[phi_] *)

Import [FileNameJoin [WD, "GLOBAL" , "Geo2_Definition_Fourierpoly.wl"] ]

(* phi_k und Startvektor initialisieren *)

phicore $=$ Table $[\mathrm{N}[\mathrm{x}, \mathrm{nprec}], \mathrm{x}, \mathrm{Pi} / 2,3 \mathrm{Pi} / 2, \mathrm{Pi} / \mathrm{np}]$;

phiall=Table [N [x,nprec] ,x, 0,2Pi-Pi/np,Pi/np] ; svalues [i_]=phiall;

(* Sin-/Costerme vorab berechnen spart viel Rechenzeit *)

$\sin 2 \mathrm{~m}=$ Chop [Table [Sin [2*m*phicore],m,1,np/2],10^-nprec]; (* Rest analog $*$ )

(* Trigonometrisches Interpolationspolynom *)

$\operatorname{pt}\left[\mathrm{t}_{-}, \mathrm{m}_{-}, \mathrm{y}_{-}\right]:=1 / \operatorname{Sqrt}[$ Dimensions $[\mathrm{y}][[1]]] *(\operatorname{Re}[\mathrm{y}[[1]]]+$ $2 * \operatorname{Sum}[(\operatorname{Re}[y[[k+1]]] \operatorname{Cos}[k * t]-\operatorname{Im}[y[[\mathrm{k}+1]]] \operatorname{Sin}[\mathrm{k} * \mathrm{t}]),\{\mathrm{k}, 1,(\mathrm{~m} / 2)-1\}]+$ $\operatorname{Re}[y[[1+\operatorname{Dimensions}[\mathrm{y}][[1]] / 2]]] \operatorname{Cos}[(\mathrm{m} / 2) * \mathrm{t}])$;

(* Routinen zum Ausdehnen der Größen von [Pi/2,3Pi/2] auf [0,2Pi) *)

oddextension [vec_] :=Flatten [Append [Prepend [vec, Table [-vec [ [np/2+1-k]] , $\{\mathrm{k}, 0, \mathrm{np} / 2-1\}]]$, Table $[-\operatorname{vec}[[\mathrm{np}-\mathrm{k}]],\{\mathrm{k}, 0, \mathrm{np} / 2-2\}]]]$;

evenextension [vec_] :=Flatten [Append [Prepend [vec, Table [vec [ [np/2+1-k] ], $\{\mathrm{k}, 0, \mathrm{np} / 2-1\}]], \mathrm{Table}[\mathrm{vec}[[\mathrm{np}-\mathrm{k}]],\{\mathrm{k}, 0, \mathrm{np} / 2-2\}]]]$;

(* Start der Iterationsschleife *)

Do $[$ to $=$ Timing [

lnRvalues $=\log [r[$ svalues $[i-1]]] ; \operatorname{sigmavalues}=r^{\prime}[$ svalues $[i-1]] / r[$ svalues $[i-1]]$;

Block [ $\{$ MinPrecision=nprecfourier, \$MaxPrecision=nprecfourier $\}$, $f k \operatorname{lnR}=$ Fourier [lnRvalues, FourierParameters $\rightarrow\{0,-1\}]$ ] ;

lnRfourier [phi_] = Expand[pt [phi,Dimensions [fklnR] [[1]],fklnR]];

anlnR=Append [Coefficient [lnRfourier [phi], Table [Cos [2m*phi], \{m,1,np/2-1\}] ], 0] ;

bnlnR=Coefficient [lnRfourier [phi] , Table [Sin [(2m-1) phi], \{m,1,np/2\}]];

(* Berechnung $\mathrm{K}[\mathrm{lnR}] / \mathrm{K}[\mathrm{v}] / \mathrm{K}[\mathrm{q}]$ nur auf $[\mathrm{Pi} / 2,3 \mathrm{Pi} / 2]$ spart Rechenzeit *)

$\mathrm{klnR} \operatorname{core}=$ Chop [Total [anlnR $\left.* \sin 2 \mathrm{~m}-\mathrm{bnlnR} * \cos 2 \mathrm{~mm} 1], 10^{\wedge}-\mathrm{nprec}\right]$;

klnRvalues=oddextension [klnRcore] ; fvalues=svalues [i-1] -phiall-klnRvalues;

vvals=ArcTan [sigmavalues] ; rvalues=Sqrt [1+sigmavalues $\left.{ }^{2}\right]$;

Block [ $\{$ MinPrecision=nprecfourier, $\$$ MaxPrecision=nprecfourier $\}$, $\mathrm{fkv}=$ Fourier [vvals, FourierParameters $\rightarrow\{0,-1\}]$ ] ;

vfourier[phi_] = Expand [pt [phi,Dimensions [fkv] [[1]],fkv]];

anv=Coefficient [vfourier [phi], Table [Cos [(2*m-1)phi] , \{m,1,np/2\}]];

bnv=Append [Coefficient [vfourier [phi] , Table [Sin [2*m*phi] , $\{\mathrm{m}, 1, \mathrm{np} / 2-1\}]], 0]$;

$\mathrm{w}=$ Chop [Total $[\mathrm{anv} * \sin 2 \mathrm{~mm} 1-\mathrm{bnv} * \cos 2 \mathrm{~m}], 10^{\wedge}$-nprec $]$; wvalues=evenextension $[\mathrm{w}]$; alpha=Chop [(1/znp) *Sum [vvals [[1] ], \{1,Dimensions [vvals] [[1] ] \}], 10^-nprec] ; $\mathrm{q}=($ fvalues $*$ sigmavalues $) /($ rvalues $* \operatorname{Exp}[$ wvalues $])$;

Block $[\{\$$ MinPrecision=nprecfourier, $\$$ MaxPrecision=nprecfourier $\}$, $f \mathrm{kq}=$ Fourier [qvalues, FourierParameters $\rightarrow\{0,-1\}]]$;

qfourier[phi_] = Expand [pt [phi,Dimensions [fkq] [[1]],fkq]];

anq=Append [Coefficient [qfourier [phi] , Table [Cos [2*m*phi] , \{m, 1,np/2-1\}] ] ,0] ; bnq=Coefficient [qfourier [phi] ,Table [Sin [(2*m-1)*phi] , \{m,1,np/2\}] ];

pcore $=$ Chop [Total [anq $* \sin 2 \mathrm{~m}-$ bnq $* \cos 2 \mathrm{~mm} 1], 10^{\wedge}$-nprec] ; $=$ oddextension [pcore]; qdach $=\operatorname{Chop}\left[(1 / \mathrm{znp}) * \operatorname{Sum}[\mathrm{q}[[1]],\{1, \operatorname{Dimensions}[\mathrm{q}][[1]]\}], 10^{\wedge}\right.$-nprec] ;

svalues $[i]=$ svalues $[i-1]-\left(\right.$ fvalues $/\left(\right.$ rvalues $\left.\left.^{2}\right)\right)-$

$(((\mathrm{p}+\mathrm{qdach} * \operatorname{Tan}[\mathrm{al}$ pha] $) * \operatorname{Exp}[$ wvalues] $) /$ rvalues $) ;]$;

to // Print, (* Ausgabe der Zeit für einen Schleifendurchgang *)

\{i, 1, niter $\}$ ]; (* Ende der Iterationsschleife *) 


\section{Kapitel 4}

\section{Anwendung konformer Koordinatentransformationen}

Studiert man physikalische Gesetzmäßigkeiten auf einem Bereich des Raumes, so ist bei der mathematischen Formulierung der Grundgesetze aus mehreren Gründen derjenigen Fassung der Vorzug zu geben, die in möglichst prägnanter Form die Zusammenhänge aufzeigt. Diesem Anliegen wird in der Theorie durch eine koordinatenfreie Verknüpfung von fundamentalen Differentialoperatoren - wie etwa der auf gewisse Zustandsgrößen wirkenden Divergenz oder des Gradienten — in Gleichungen Rechnung getragen.

Durch eine solche Vorgehensweise wird schlußendlich auch der Forderung nach Invarianz der Gleichungen bezüglich beliebiger Bezugssysteme entsprochen, ein Umstand, dem die Überlegung zu Grunde liegt, daß die Vorgänge in der Natur auch ohne Koordinaten ablaufen und letztere demnach für den Kern der Dinge unwesentlich sind. Die Bedeutung der Koordinaten reduziert sich in dieser Sichtweise auf die Rolle von - a priori nicht notwendigen - Zahlenwerten, zu interpretieren als Adressen bzw. Richtungen, die dem Ort bzw. tensoriellen Zustandsgrößen im jeweiligen System zugeteilt werden.

Die Praxis der numerischen Simulation im Sinne eines number crunchings erfordert allerdings die Bezugnahme auf eben ein solches Koordinatensystem, und ein konkretes wird unter Berücksichtigung bestmöglicher Recheneffizienz und geometrischen Erwägungen einzuführen sein. Die Gestalt der Bildungsgesetze bei Bezugnahme auf ein spezielles Koordinatensystem und anschließender Koordinatentransformation - man denke an die Form des Laplace-Operators bei Übergang von kartesischen Koordinaten zu Polarkoordinaten - ist dabei selbstverständlich Änderungen unterworfen. Man wird diese Änderungen aber, von der invarianten Formulierung her kommend, vielmehr als von Raumpunkt zu Raumpunkt erfolgende Änderung der metrischen Verhältnisse zu interpretieren wissen, sie über den Maßstensor quantifizieren und so der Simulation zugänglich machen. Von der kartesischen Parametrisierung des Raumes ausgehend fließen dann in die Gesetze nach erfolgter Transformation gewissermaßen geometriebedingte Korrekturterme ein.

In weiter Folge wird konsequent vom Summationsübereinkommen nach EinsteIN Gebrauch gemacht, welches der Übersichtlichkeit sehr entgegenkommt. Dabei wird vereinbart, daß bei sich über die Raumdimension erstreckenden Summenbildungen, innerhalb derer ein Laufindex genau zweimal - und zwar einmal hochgestellt und einmal tiefgestellt - auftritt, das Summationszeichen unterdrückt wird. So setzt man beispielsweise abkürzend

$$
X_{i} X^{i}=\sum_{i=1}^{n} X_{i} X^{i}, \quad \frac{\partial V^{i}}{\partial x_{i}}+\Gamma_{j i}^{i} V^{j}=\sum_{i=1}^{n} \frac{\partial V^{i}}{\partial x_{i}}+\sum_{j=1}^{n} \sum_{i=1}^{n} \Gamma_{j i}^{i} V^{j}, \quad \text { etc... }
$$

Die Ausführungen der beiden ersten Abschnitte folgen in Notation und Inhalt [8]. 


\subsection{Der Riemannsche Raum}

Obige Aspekte vor Augen, legen wir den folgenden Betrachtungen die Konzeption eines Riemannschen Raumes $\mathcal{R}$ zu Grunde, als dessen Ausgangspunkt eine mit Hausdorff-Topologie ${ }^{1}$ versehene $^{2}$ Punktmenge $\mathcal{M}$ anzusehen ist, auf der physikalische Gesetze untersucht werden sollen und die den eingangs angesprochenen räumlichen Bereich repräsentierend verallgemeinern soll.

Die zunächst völlig abstrakte Menge $\mathcal{M}$ wird zu einer differenzierbaren Mannigfaltigkeit, indem sie durch Einführung des Kartenbegriffs durch Homöomorphismen lokal parametrisiert wird: Bezeichne $\mathcal{T}_{1}$ eine geeignete Topologie des $\mathbb{R}^{n}, \mathcal{T}_{2}$ eine solche auf $\mathcal{M}$, so wird für je zwei offene Mengen $\mathcal{O}_{1} \in \mathcal{T}_{1}, \mathcal{O}_{2} \in \mathcal{T}_{2}$ durch eine bijektive und im topologischen Sinne in beide Richtungen stetige Abbildung $\kappa: \mathcal{O}_{1} \rightarrow \mathcal{O}_{2}$ ein lokales Koordinatensystem errichtet und somit ist man in der Lage, jedem Element $P \in \mathcal{O}_{2}$ vermöge $P=\kappa\left(\xi_{1}, \ldots, \xi_{n}\right)$ wohlbestimmte Koordinaten in Form eines $n$-Tupels $\boldsymbol{\xi}=\left(\xi_{1}, \ldots, \xi_{n}\right)$ zuzuordnen. Der wesentliche Punkt bei dieser Konstruktion besteht also in der Herstellung einer topologischen Verknüpfung von $\mathcal{M}$ mit dem $\mathbb{R}^{n}$, auf dem die Werkzeuge der Analysis zur Verfügung stehen. Anders als im affinen Raum kann aber der Fall der Unmöglichkeit einer globalen Parametrisierung von $\mathcal{M}$ eintreten.

Werden zwei nicht-disjunkte offene Mengen $\mathcal{O}, \overline{\mathcal{O}} \in \mathcal{T}_{2}$ durch die Karten $\kappa$ bzw. $\bar{\kappa}$ parametrisiert, dann bezeichnet man bei Einschränkung des Definitionsbereiches der beiden Karten auf die Urbilder des Durchschnittes die Abbildung $\bar{\kappa}^{-1} \circ \kappa$ als Koordinatentransformation, so es sich um einen Diffeomorphismus handelt. Ein solcher Kartenwechsel hat demnach zusätzlich zur Homöomorphie auch noch in beide Richtungen Differenzierbarkeitseigenschaften auf zwei speziellen Teilmengen des $\mathbb{R}^{n}$, die beide auf verschiedene Weise den Durchschnitt $\mathcal{O} \cap \overline{\mathcal{O}}$ parametrisieren, aufzuweisen.

Durch jede feste, aber beliebige Wahl einer Karte $\kappa$ und eines $n$-Tupels $\boldsymbol{h} \in \mathbb{R}^{n}$ wird in einer geeigneten Umgebung um einen Punkt $P \in \mathcal{M}$ mit den Koordinaten $\boldsymbol{\xi}_{0}$ durch die Setzung

$$
\gamma(t)=\kappa\left(\boldsymbol{\xi}_{0}+\left(t-t_{0}\right) \boldsymbol{h}\right)
$$

ein Kurvenstück $\mathcal{C}$ auf $\mathcal{M}$ dergestalt parametrisiert, daß auf dem gesamten Definitionsbereich des Kurvenparameters $t \in\left[t_{0}-\delta_{1}, t_{0}+\delta_{2}\right]$ als jeweiliger Tangentenvektor entlang $\kappa^{-1}(\mathcal{C})$ im Sinne einer Abbildung eines Intervalls in den $\mathbb{R}^{n}$ immer konstant das $n$-Tupel $\boldsymbol{h}$ resultiert, das auch für den Punkt $P$ selbst einen Tangentenvektor repräsentiert. Man sieht dies unmittelbar durch Differentiation nach dem Parameter $t$. Läßt man nun $\boldsymbol{h}$ den $\mathbb{R}^{n}$ durchlaufen, so bildet die Gesamtheit der Tangentenvektoren an alle Kurven durch den Punkt $P$ einen linearen Vektorraum, nämlich den Tangentialraum $\mathcal{T}_{P}(\mathcal{M})$ von $\mathcal{M}$ im Punkt $P$, der auf Grund der Konstruktion dem $\mathbb{R}^{n}$ isomorph ist und somit eine Identifizierung erlaubt.

Speziell wird man über die Setzungen $\boldsymbol{h}=\boldsymbol{e}_{\boldsymbol{i}}, i=1, \ldots n$, auf die sogenannten Koordinatenlinien der Karte geführt, die sich auf Grund des Umstandes, daß es sich bei $\kappa$ um einen Homöomorphismus handelt, im Punkt $P$ - parametrisiert durch $\gamma\left(t_{0}\right)$ - schneiden müssen und nach obigem durch die $n$ Kurven

$$
\gamma_{i}(t)=\kappa\left(\boldsymbol{\xi}_{0}+\left(t-t_{0}\right) \boldsymbol{e}_{\boldsymbol{i}}\right)
$$

gegeben sind. Damit ist für den Tangentialraum $\mathcal{T}_{P}(\mathcal{M})$ eine Basis $\left\{\boldsymbol{e}_{i}\right\}$ in Form der Tangentenvektoren an die Koordinatenlinien gefunden, wobei zu beachten ist, daß letztere von der speziellen Wahl der Karte $\kappa$ abhängen. Wählt man jetzt einen Vektor $\boldsymbol{v}$ aus dem Tangentialraum, so führt seine Darstellung als Linearkombination der Basisvektoren bezüglich verschiedener Karten zu verschiedenen $n$-Tupeln und damit entsprechen ein und demselben Vektor $\boldsymbol{v}$ verschiedene Vektoren des $\mathbb{R}^{n}$. Eine Abhilfe läßt sich jedoch sofort durch den Übergang zum kartesischen Produkt $\mathbb{R}^{n} \times \mathfrak{K}$ unter Aufnahme der Menge aller zulässigen Karten und anschließender Definition einer Äquivalenzrelation erzielen, wodurch die Eindeutigkeit der Darstellung der Tangentenvektoren sichergestellt wird [8, S.284]. Damit ist der Begriff von Punktkoordinaten auf einer Mannigfaltigkeit geklärt.

\footnotetext{
${ }^{1}$ Neben den drei bekannten, an eine Topologie gestellten axiomatischen Forderungen gilt hier auch noch das Trennungsaxiom T2: Alle paarweise verschiedenen Punkte können durch (disjunkte) Umgebungen separiert werden.
} 
Ein Skalarfeld $\omega: \mathcal{M} \rightarrow \mathbb{R}$ als reellwertige Funktion auf einer Mannigfaltigkeit läßst sich durch den Kartenbegriff unmittelbar durch die Forderung erklären, daß die Zusammensetzung mit jeder Karte $\kappa$ eine differenzierbare Funktion $\omega \circ \kappa: \mathbb{R}^{n} \rightarrow \mathbb{R}$ ergibt, wobei offenbar wiederum auf den $\mathbb{R}^{n}$ zurückgegriffen wird. Über die Definition der Richtungsableitung eines Skalarfeldes $\omega$ in Richtung eines Vektors $\boldsymbol{v}=V^{i} \boldsymbol{e}_{\boldsymbol{i}} \in \mathcal{T}_{P}(\mathcal{M})$,

$$
\boldsymbol{v}(\omega)=\frac{\partial(\omega \circ \kappa)}{\partial x_{i}} V^{i}=\partial_{i}(\omega \circ \kappa) V^{i},
$$

ergeben sich folglich die Richtungsableitungen von $\omega$ in Richtung der Tangentenvektoren $\boldsymbol{e}_{\boldsymbol{j}} \mathrm{zu}$

$$
\boldsymbol{e}_{j}(\omega)=\frac{\partial(\omega \circ \kappa)}{\partial x_{i}} \delta_{j}^{i}=\frac{\partial(\omega \circ \kappa)}{\partial x_{j}}=\partial_{j}(\omega \circ \kappa),
$$

und somit lassen sich die Basisvektoren auch in der Form $\partial_{i}, i=1, \ldots n$, anschreiben. Bei einer Koordinatentransformation, wie sie durch die Angabe eines bijektiven vektoriellen Zusammenhanges $\overline{\boldsymbol{x}}(\boldsymbol{x})=f(\boldsymbol{x})$ bzw. $\boldsymbol{x}(\overline{\boldsymbol{x}})=f^{-1}(\overline{\boldsymbol{x}})$ vermittelt wird, fließt in die noch anzugebenden tensoriellen Transformationsgesetze unter Berücksichtigung der ko- oder kontravarianten Natur einer Größe jeweils die Matrix der partiellen Differentialquotienten bzw. deren Inverse ein. Das Summationsübereinkommen bleibt bei allen diesen Produkten in dem Sinne aufrecht, als in diesem speziellen Fall die Indizes im Zähler der Transformationsmatrix hochgestellt, jene im Nenner hingegen tiefgestellt zu denken sind. Für die nachfolgende Formel bedeutet dies eine Summation über den Index $j$. Die Maßvektoren transformieren sich dann bei einem Kartenwechsel, wie er durch $\overline{\boldsymbol{x}}=\bar{\kappa}^{-1} \circ \kappa(\boldsymbol{x})$ geleistet wird, kogredient entsprechend

$$
\bar{\partial}_{i}=\frac{\partial x_{j}}{\partial \bar{x}_{i}} \partial_{j} \quad \text { bzw. } \quad \partial_{i}=\frac{\partial \bar{x}_{j}}{\partial x_{i}} \bar{\partial}_{j} .
$$

Die Entwicklung der Transformationsgesetze für Tensorfelder beliebiger Stufe auf $\mathcal{M}$ erfordert die Klärung des ko- und kontravarianten Tensorbegriffs. Dazu konstruiert man in jedem Punkt der Mannigfaltigkeit den zum Tangentialraum $\mathcal{T}_{P}(\mathcal{M})$ dualen Vektorraum $\mathcal{T}_{P}^{*}(\mathcal{M})$ als Menge der Linearformen $\alpha: \mathcal{T}_{P}(\mathcal{M}) \rightarrow \mathbb{R}$ mit der zu $\left\{\partial_{i}\right\}$ dualen Basis $\left\{d x_{i}\right\}$, die $d x_{i}\left(\partial_{j}\right)=\delta_{j}^{i}$ leistet. Der als Kotangentialraum bezeichnete Vektorraum ist mit seinem Tangentialraum in natürlicher Weise über ein Skalarprodukt verknüpft, das man unter Berücksichtigung der Linearität durch

$$
\langle\alpha, \boldsymbol{v}\rangle:=\alpha(\boldsymbol{v})=\left\langle A_{i} d x_{i}, V^{j} \partial_{j}\right\rangle=A_{i} V^{j} d x_{i}\left(\partial_{j}\right)=A_{i} V^{i}
$$

einführt. In der Darstellung einer Linearform $\alpha$ sind die Basisvektoren des Kotangentialraumes $d x_{i}$ stets mit hochgestellten Indizes zu denken und somit ist zu summieren. Tiefgestellte Koordinatenindizes wie in $\alpha=A_{i} d x_{i}$ charakterisieren kovariante Größen, hochgestellte wie in $\boldsymbol{v}=V^{i} \partial_{i}$ sind kontravariante Koordinaten. Die vorerst nur im Punkt $P$ gegebenen Begriffsbildungen lassen sich durch Differenzierbarkeitsforderungen leicht auf ganz $\mathcal{M}$ ausdehnen: Stellt das von Punkt zu Punkt variierende Skalarprodukt $\langle\alpha(P), \boldsymbol{v}(P)\rangle$ ein Skalarfeld auf $\mathcal{M}$ dar, so gelangt man mit der Menge der differenzierbaren Linearformen zum Begriff $\boldsymbol{v}^{*}(\mathcal{M})$ der kovarianten Tensorfelder erster Stufe, wobei im Skalarprodukt nur differenzierbare Vektorfelder — im Sinne der Richtungsableitung bezüglich beliebiger Skalarfelder auf $\mathcal{M}$ - zulässig sind, die ihrerseits die mit $\boldsymbol{v}(\mathcal{M})$ bezeichnete Menge der kontravarianten Tensorfelder erster Stufe darstellen. Die Menge der Skalarfelder auf $\mathcal{M}$ selbst konstituiert die invarianten Tensorfelder der Stufe Null und sei mit $\mathbb{F}$ bezeichnet.

Mit diesen Definitionen ist man in der Lage, über die kartesischen Produkte ko- oder kontravarianter Vektorfelder Tensorfelder von höherer als erster Ordnung einzuführen. Verallgemeinernd nennt man eine multilineare Abbildung

$$
\varphi: \underbrace{\boldsymbol{v}^{*} \times \cdots \times \boldsymbol{v}^{*}}_{k} \times \underbrace{\boldsymbol{v} \times \cdots \times \boldsymbol{v}}_{m} \rightarrow \mathbb{F}
$$

ein $k$-fach kontravariantes und $m$-fach kovariantes Tensorfeld der Stufe $k+m$, dessen $n^{k+m}$ Koordinaten bezüglich einer Karte $\kappa$ in einem Punkt $P$ durch Einsetzen sämtlicher Basisvektoren im Sinne von

$$
\Phi_{j_{1} \cdots j_{m}}^{i_{1} \cdots i_{k}}=\varphi\left(d x_{i_{1}}, \ldots, d x_{i_{k}}, \partial_{j_{1}}, \ldots, \partial_{j_{m}}\right)
$$


ermittelt werden können. Bei Übergang zu einem anderen Koordinatensystem transformieren sich die Tensorkoordinaten dabei gemäß dem allgemeinen Gesetz

$$
\bar{\Phi}_{j_{1} \cdots j_{m}}^{i_{1} \cdots i_{k}}=\frac{\partial \bar{x}_{i_{1}}}{\partial x_{h_{1}}} \cdots \frac{\partial \bar{x}_{i_{k}}}{\partial x_{h_{k}}} \frac{\partial x_{l_{1}}}{\partial \bar{x}_{j_{1}}} \cdots \frac{\partial x_{l_{m}}}{\partial \bar{x}_{j_{m}}} \Phi_{l_{1} \cdots l_{m}}^{h_{1} \cdots h_{k}} .
$$

Bemerkenswert an der obigen Konstruktion von Tensorfeldern beliebiger Stufe ist der Umstand, daß kontravariante Tensorfelder erster Stufe zunächst als lineare Abbildungen $\boldsymbol{V}: \boldsymbol{v}^{*} \rightarrow \mathbb{F}$ eingeführt werden, die auf der Menge der differenzierbaren Linearformen wirken und als solche den Bidualraum bilden. Auf Grund der implizit vorausgesetzten Endlichdimensionalität aller beteiligter Vektorräume ist wegen der dann geltenden Reflexivität der Bidualraum $\boldsymbol{v}^{* *}$ dem ursprünglichen Raum $\boldsymbol{v}$ der Vektorfelder isomorph, wodurch eine Identifizierung ihrer Elemente gestattet ist.

Durch die Forderung nach einem inneren Produkt im Tangentialraum $\mathcal{T}_{P}(\mathcal{M})$ eines beliebigen Punktes $P \in \mathcal{M}$ wird die differenzierbare Mannigfaltigkeit schließlich zu einem Riemannschen Raum $\mathcal{R}$, in dem im Gegensatz zu den Verhältnissen, wie sie in einem euklidischen Raum vorliegen, die Basisvektoren von Punkt zu Punkt variieren können. Richtet man sein Augenmerk auf eben eine solche lokale Basis, so wird durch die Setzung

$$
g_{i j}(P):=\left(\partial_{i}, \partial_{j}\right)(P)
$$

ein kovariantes Tensorfeld 2. Stufe induziert, das zur Beschreibung der geometrischen Verhältnisse auf $\mathcal{R}$ herangezogen werden kann. Die als metrische Fundamentalform bezeichnete Abbildung ist symmetrisch und transformiert sich kogredient, wie man durch Einsetzen in 4.2 oder unmittelbar gemäß 4.3) einsieht,

$$
\bar{g}_{i j}(P)=\frac{\partial x_{k}}{\partial \bar{x}_{i}} \frac{\partial x_{l}}{\partial \bar{x}_{j}} g_{k l}(P) .
$$

Der damit eingeführte kovariante Maßtensor ist in der vorliegenden Arbeit von zentraler Bedeutung und bildet nicht zuletzt den Unterbau für alle numerisch durchzuführenden Transformationen.

Der kontravariante Maßtensor $g^{i j}$ wird entsprechend über das im Dualraum induzierte innere Produkt der dortigen Basisformen erklärt. Unter Verzicht einer Angabe seines Transformationsgesetzes bemerken wir, daß er bei Kenntnis von $g_{i j}$ in beliebigen Koordinaten, wie sie ja durch Vorliegen einer $n \times n$-Matrix gegeben ist, sofort aus dem Zusammenhang $g^{i k} g_{k j}=\delta_{j}^{i}$ durch Invertierung hergeleitet werden kann. Durch eine nicht von vorneherein auszuschließende Singularität des Maßtensors wird man offensichtlich in eine ziemlich prekäre Lage versetzt - ein durchaus nicht pathologischer Sachverhalt, wie man am Beispiel des Übergangs zu Polarkoordinaten ersieht. So bringt der Verlust der Bijektivität der Koordinatentransformation unter anderem die Unmöglichkeit einer eindeutigen Zuordnung von Vektorkoordinaten im Punkt der Koordinatensingularität mit sich, woraus Schwierigkeiten bei Diskretisierungsprozessen resultieren.

Durch den Maßtensor in seiner kovarianten Form ist die Möglichkeit gegeben, die kontravariante Koordinate einer tensoriellen Größe in eine kovariante umzurechnen und umgekehrt errechnet man kontravariante Koordinaten durch Verjüngung mit dem kontravarianten Fundamentaltensor aus kovarianten, wie beispielsweise aus $V_{i}=g_{i j} V^{j}$ oder $A_{k}^{i j}=g^{i h} g^{j l} A_{h l k}$ ersichtlich wird. Ein solches Hinauf- oder Herunterziehen von Indizes kann sich speziell bei einer rein ko- oder kontravarianten Formulierung von Gesetzen als hilfreich erweisen.

Der Körper der komplexen Zahlen $\mathbb{C}$ kann mit diesen Ausführungen nun auch als Riemannsche Mannigfaltigkeit aufgefaßt werden, die eine globale Parametrisierung gestattet. An die Stelle des bisher betrachteten Gebietes $\mathfrak{G} \subseteq \mathbb{C}$ tritt davon abstrahierend eine offene Punktmenge, wobei der Tangentialraum in jedem Punkt dem $\mathbb{R}^{2}$ isomorph ist. Darüberhinaus wird durch diese Herangehensweise der saubere Übergang zu krummlinigen Koordinaten ermöglicht: Ausgehend von einer kartesischen Parametrisierung der Punktmenge, bei der der Fundamentaltensor in jedem Punkt konstant in Form der $2 \times 2$-Einheitsmatrix in Erscheinung tritt, folgen bei Kenntnis des Bildungsgesetzes des Kartenwechsels seine neuen Koordinaten unmittelbar aus 4.4. 


\subsection{Differentialoperatoren im Riemannschen Raum}

Ist $\omega$ ein Skalarfeld auf $\mathcal{R}$, so wird durch Gleichung 4.1) eine im zu $\mathcal{T}_{P}(\mathcal{M})$ dualen Vektorraum gelegene Linearform $d \omega \in \mathcal{T}_{P}^{*}(\mathcal{M})$ definiert, deren kanonische Darstellung bezüglich einer Karte $\kappa$

$$
d \omega=\frac{\partial(\omega \circ \kappa)}{\partial x_{i}} d x_{i}
$$

als Gradient bezeichnet wird, wobei die Koordinatendifferentiale $d x_{i}$ als duale Basis des Kotangentialraumes aufzufassen sind und somit nach deren Konstruktion die Beziehungen $d x_{i}\left(\partial_{j}\right)=\delta_{j}^{i}$ gelten. Synonym dazu wird auch die Notation mittels Nabla-Operator verwendet, $d \omega=\nabla \omega$. Setzt man in Gleichung (4.5) einen Vektor $\boldsymbol{h}$ als Argument, so erhält man (4.1) auf Grund von $d x_{i}(\boldsymbol{h})=h^{i}$ zurück. In dieser Form erkennt man in $d \omega$ ein kovariantes Tensorfeld erster Stufe, dessen Koordinaten sich gemäß

$$
\frac{\partial(\omega \circ \bar{\kappa})}{\partial \bar{x}_{i}}=\frac{\partial x_{j}}{\partial \bar{x}_{i}} \frac{\partial(\omega \circ \kappa)}{\partial x_{j}}
$$

transformieren. Wir bemerken hierzu noch, daß der in Gleichung (4.5) auf ein Skalarfeld wirkende Operator $d$ in der Theorie der schiefsymmetrischen kovarianten Tensorfelder, der $n$-Formen, als äußeres Differential bezeichnet wird, durch dessen Anwendung im allgemeinen Fall eine $n$-Form in eine $(n+1)$-Form überführt wird.

Bei Kenntnis der Koordinaten des Maßtensors bezüglich eines speziellen Bezugssystems leitet man aus diesem die Koeffizienten des Riemannschen Zusammenhanges über

$$
\Gamma_{j k}^{i}=\frac{1}{2} g^{i l}\left(\frac{\partial g_{l j}}{\partial x_{k}}+\frac{\partial g_{l k}}{\partial x_{j}}-\frac{\partial g_{j k}}{\partial x_{l}}\right)
$$

ab. Der in diese Summe eingehende kontravariante Maßtensor hat im Sinne des inneren Produktes im Dualraum als Argumente je zwei der dualen Basisvektoren und es gelten die Beziehungen $g^{i k} g_{k j}=$ $\delta_{j}^{i}$. Unter Verwendung des Kofaktors ${ }^{2}$ berechnen sich seine Koordinaten mittels $g^{i j}=K o f\left(g_{i j}\right) / g$, wenn dabei mit $g=\operatorname{det}\left(g_{i j}\right)$ die Determinante des kovarianten Fundamentaltensors bezeichnet wird.

Die Divergenz eines kontravarianten Vektorfeldes $\boldsymbol{v}$ wird nun in Riemannschen Räumen unter Verwendung der Zusammenhangskoeffizienten durch die Setzung

$$
\operatorname{div} \boldsymbol{v}=\frac{\mathfrak{d} v^{i}}{\mathfrak{d} x_{i}}=\frac{\partial v^{i}}{\partial x_{i}}+\Gamma_{j i}^{i} v^{j}
$$

eingeführt. Bei Verwendung kartesischer Koordinaten leistet der zweite Summand keinen Beitrag, da sich der Maßtensor in jedem Punkt als Einheitsmatrix präsentiert und somit sämtliche Zusammenhangskoeffizienten in 4.6 identisch verschwinden. Dies steht in Übereinstimmung mit der in euklidischen Räumen eingeführten Divergenz unter Bezugnahme auf ein rechtwinkeliges Orthonormalsystem. Im Falle allgemeiner Koordinaten erkennt man in diesem Summanden jenen Korrekturterm, der unter Berücksichtigung der lokalen geometrischen Verhältnisse zur klassischen Divergenz zu addieren ist. Man sieht, daß die sogenannte kovariante Ableitung die partielle Differentiation in affinen Räumen in folgendem Sinne verallgemeinert: In Abhängigkeit der ko- oder kontravarianten Natur einer Koordinate sind geometriebedingt Terme zu subtrahieren oder zu addieren, und zwar für eine beliebige tensorielle Größe nach dem allgemeinen Bildungsgesetz

$$
\frac{\mathfrak{d} \Phi_{j_{1} \cdots j_{m}}^{i_{1} \cdots i_{n}}}{\mathfrak{d} x_{p}}=\frac{\partial \Phi_{j_{1} \cdots j_{m}}^{i_{1} \cdots i_{n}}}{\partial x_{p}}+\sum_{h=1}^{n} \Gamma_{l p}^{i_{h}} \Phi_{j_{1} \cdots j_{m}}^{i_{1} \cdots l i_{n}}-\sum_{k=1}^{m} \Gamma_{j_{k} p}^{l} \Phi_{j_{1} \cdots \cdots j_{m}}^{i_{1} \cdots i_{n}},
$$

\footnotetext{
${ }^{2}$ Der Kofaktor eines Matrixelementes $a_{i j}$ ist das Produkt $K o f\left(a_{i j}\right)=(-1)^{i+j} \operatorname{det}\left(A_{j i}\right)$, wobei mit $A_{j i}$ jene Matrix bezeichnet ist, die nach Streichung der $j$-ten Zeile und $i$-ten Spalte resultiert.
} 
wobei durch die kovariante Differentiation die kovariante Stufe eines Tensors um eine Stufe erhöht wird. Wir weisen auf die Produktregel der kovarianten Ableitung hin, es gilt für zwei Tensoren $\varphi$ und $\boldsymbol{\psi}$ beliebiger Stufe in Analogie zur bekannten Produktregel

$$
\frac{\mathfrak{d}\left(\Phi_{j_{1} \cdots j_{m}}^{i_{1} \cdots i_{n}} \Psi_{k_{1} \cdots k_{q}}^{h_{1} \cdots h_{p}}\right)}{\mathfrak{d} x_{l}}=\frac{\mathfrak{d} \Phi_{j_{1} \cdots j_{m}}^{i_{1} \cdots i_{n}}}{\mathfrak{d} x_{l}} \Psi_{k_{1} \cdots k_{q}}^{h_{1} \cdots h_{p}}+\Phi_{j_{1} \cdots j_{m}}^{i_{1} \cdots i_{n}} \frac{\mathfrak{d} \Psi_{k_{1} \cdots k_{q}}^{h_{1} \cdots h_{p}}}{\mathfrak{d} x_{l}} .
$$

In Gleichung 4.7) kann man sich aber auch auf folgendem Weg von den Zusammenhangskoeffizienten frei machen: Obwohl es sich bei diesen Größen auf Grund ihres Transformationsgesetzes um keine Tensoren handelt, lassen sich dennoch einige Rechenregeln ${ }^{3}$ übertragen, es folgt

$$
2 g \Gamma_{j i}^{i}=g\left(\Gamma_{j i}^{i}+\Gamma_{j k}^{k}\right)=g g^{i k}\left(\Gamma_{k j i}+\Gamma_{i j k}\right)=g g^{i k} \frac{\partial g_{i k}}{\partial x_{j}}=\operatorname{Kof}\left(g_{i k}\right) \frac{\partial g_{i k}}{\partial x_{j}}=\frac{\partial g}{\partial x_{j}},
$$

wobei in der letzten Gleichung die Kettenregel zur Anwendung gebracht wurde. Somit bestimmt sich die Divergenz unter Verwendung beliebiger Koordinaten unter alleiniger Zuhilfenahme des Maßtensors aus

$$
\operatorname{div} \boldsymbol{v}=\frac{\partial V^{i}}{\partial x_{i}}+\frac{1}{2 g} \frac{\partial g}{\partial x_{j}} V^{j}=\frac{\partial V^{i}}{\partial x_{i}}+\frac{\partial(\ln \sqrt{|g|})}{\partial x_{j}} V^{j}=\frac{1}{\sqrt{|g|}} \frac{\partial\left(\sqrt{|g|} V^{i}\right)}{\partial x_{i}}
$$

Neben dem Gradienten eines Skalarfeldes und der Divergenz eines (kontravarianten) Vektorfeldes wird noch ein dritter Operator benötigt, der Laplace-Beltrami-Operator, der den LaplaceOperator in euklidischen Räumen verallgemeinert und in unserem Fall ebenfalls auf ein Skalarfeld wirkt. Bildet man die Divergenz des Gradienten unter Berücksichtigung der kovarianten Natur seiner Koordinaten, so führt dies mit 4.5 und 4.9) auf

$$
\triangle \omega=\frac{\partial\left(g^{i j} \frac{\partial \omega}{\partial x_{i}}\right)}{\partial x_{j}}+\frac{1}{2 g} \frac{\partial g}{\partial x_{j}} g^{i j} \frac{\partial \omega}{\partial x_{i}}=g^{i j} \frac{\partial^{2} \omega}{\partial x_{i} \partial x_{j}}+\left(\frac{\partial g^{i j}}{\partial x_{j}}+\frac{g^{i j}}{2 g} \frac{\partial g}{\partial x_{j}}\right) \frac{\partial \omega}{\partial x_{i}} .
$$

\subsection{Metrische Größen der konformen Abbildung}

Wir gehen jetzt an die Betrachtung der in Kapitel 2 vorgestellten konformen Abbildung, vor allem interessieren wir uns für die zusammengesetzte Abbildung $f=g \circ h: \mathfrak{Q} \rightarrow \mathfrak{G}$, die das Einheitsquadrat über den Einheitskreis konform auf ein einfach zusammenhängendes Gebiet abbildet. Die Funktion $f(z)=\zeta$ wird jetzt als Kartenwechsel zu interpretieren sein, von speziellem Interesse sind der Maßstensor und die Gestalt der oben betrachteten Differentialoperatoren (4.5), (4.7) und (4.10).

Ausgangspunkt der Überlegungen ist die Auffassung von $\mathfrak{G}$ als offene Teilmenge des zweidimensionalen Riemannschen Raumes $\mathbb{C}$, der mit Hilfe der Tupel $\zeta=(\xi, \eta)$ durch kartesische Koordinaten und der Karte $\kappa_{\zeta}$ parametrisiert sei, sodaß der Maßtensor also in jedem Punkt $P \in \mathfrak{G}$ konstant in Gestalt der $2 \times 2$-Einheitsmatrix zu Tage tritt. Ganz allgemein gilt mit zwei Karten $\kappa_{z}$ bzw. $\kappa_{\zeta}$ für einen Punkt $P \in \mathfrak{G}$ die Darstellung $P=\kappa_{z}(x, y)=\kappa_{\zeta}(\xi, \eta)$, somit kann über die Beziehungen

$$
z=\left(\kappa_{z}^{-1} \circ \kappa_{\zeta}\right)(\xi, \eta) \quad \zeta=\left(\kappa_{\zeta}^{-1} \circ \kappa_{z}\right)(x, y)
$$

auf die Rolle der konformen Abbildung $f$ bei der Koordinatentransformation geschlossen werden und man erhält folgende zentrale Aussage im

\footnotetext{
${ }^{3}$ Wir machen vom Herauf- und Herunterziehen des ersten Index, der Symmetrie in den letzten beiden Indizes und der Beziehung $\Gamma_{i j k}+\Gamma_{k j i}=\partial g_{i k} / \partial x_{j}$ Gebrauch. Weiters folgt auf Grund des Umstandes, daß der Kofaktor unabhängig von seinem assoziierten Matrixelement ist, $\operatorname{Kof}\left(g_{i k}\right)=\partial g / \partial g_{i k}$.
} 
Satz (Parametrisierung eines einfach zusammenhängenden Gebietes durch das Einheitsquadrat). Ist $\zeta=f(z)$ die konforme Abbildung vom Einheitsquadrat auf das Urbild eines durch kartesische Koordinaten $(\xi, \eta)$ parametrisierten Gebietes, so wird durch $z=f^{-1}(\zeta)$ ein Kartenwechsel vermittelt, der der Einführung orthogonaler, krummliniger Koordinaten $(x, y)$ entspricht. Die Koordinaten des kovarianten Maßtensors in einem Punkt $P$ lauten dann bezüglich der Karte $\kappa_{z}$

$$
g_{11}=g_{22}=\xi_{x}^{2}+\eta_{x}^{2}=\left|f^{\prime}(z)\right|^{2}=\frac{1}{\left|f^{-1}(\zeta)^{\prime}\right|^{2}}, \quad g_{12}=g_{21} \equiv 0
$$

und der Laplace-Operator erhält in den neuen Koordinaten die Gestalt

$$
\triangle \omega=\frac{1}{\xi_{x}^{2}+\eta_{x}^{2}}\left(\frac{\partial^{2} \omega}{\partial x^{2}}+\frac{\partial^{2} \omega}{\partial y^{2}}\right)
$$

Durch die Vorschrift $z=(x, y)=f^{-1}(\zeta)=\left(\kappa_{z}^{-1} \circ \kappa_{\zeta}\right)(\xi, \eta)$, bei der es sich klarerweise um einen Diffeomorphismus handelt, wird $\mathfrak{G}$ durch das Einheitsquadrat parametrisiert, die Koordinaten der neuen Basisvektoren bezüglich der alten, kartesischen Basis findet man sofort mit Hilfe von (4.2),

$$
\partial_{x}=\xi_{x} \partial_{\xi}+\eta_{x} \partial_{\eta}, \quad \partial_{y}=\xi_{y} \partial_{\xi}+\eta_{y} \partial_{\eta}
$$

Nun gelten aber auch die Cauchy-Riemannschen Differentialgleichungen, $\xi_{x}=\eta_{y}, \xi_{y}=-\eta_{x}$, und damit erscheinen die Koordinaten der neuen Basisvektoren als Spalten der Jacobi-Matrix $\boldsymbol{J}$,

$$
\left(\begin{array}{ll}
\partial_{x} & \partial_{y}
\end{array}\right)=\left(\begin{array}{ll}
\partial_{\xi} & \partial_{\eta}
\end{array}\right) \cdot\left(\begin{array}{cc}
\xi_{x} & -\eta_{x} \\
\eta_{x} & \xi_{x}
\end{array}\right)=\left(\begin{array}{ll}
\partial_{\xi} & \partial_{\eta}
\end{array}\right) \cdot \boldsymbol{J}
$$

woraus man auch schon deren Orthogonalität erkennt. Die neuen Koordinaten des kovarianten Maßtensors findet man aus (4.4), seine Matrix hat Diagonalgestalt und errechnet sich aus

$$
\boldsymbol{G}=\boldsymbol{J}^{\dagger} \cdot \boldsymbol{J} \cdot \boldsymbol{E}=\left(\begin{array}{cc}
\xi_{x} & \eta_{x} \\
-\eta_{x} & \xi_{x}
\end{array}\right)\left(\begin{array}{cc}
\xi_{x} & -\eta_{x} \\
\eta_{x} & \xi_{x}
\end{array}\right)=\left(\begin{array}{cc}
\xi_{x}^{2}+\eta_{x}^{2} & 0 \\
0 & \xi_{x}^{2}+\eta_{x}^{2}
\end{array}\right)
$$

Aus dieser Darstellung wird insbesondere ersichtlich, daß es sich bei der konformen Abbildung um eine Drehstreckung handelt, die die beiden lokalen Basisvektoren im selben Ausmaß, nämlich um den Faktor $\left|f^{\prime}(z)\right|=\left|\xi_{x}+i \eta_{x}\right|=\sqrt{\xi_{x}^{2}+\eta_{x}^{2}}=\sqrt{g_{i i}}$, der selbstverständlich von Punkt zu Punkt variieren kann, streckt. Beim Übergang zur Exponentialdarstellung bzw. unter Verwendung des inneren Produktes, das man auf die Vektoren $\partial_{\xi}$ und $\partial_{x}$ wirken läßt, errechnet man den Drehwinkel

$$
\theta=\arg \left(f^{\prime}(z)\right)=\arctan \frac{\eta_{x}}{\xi_{x}}=\arccos \frac{\xi_{x}}{\sqrt{\xi_{x}^{2}+\eta_{x}^{2}}}
$$

Die Transformation des Laplace-Operators bestätigt man durch Nachrechnen, indem man 4.10 heranzieht. Dabei gilt offensichtlich $\sqrt{|g|}=g_{11}=g_{22}$.

Die Koeffizienten des Riemannschen Zusammenhangs (CHRISTOFFELsche Symbole 2. Art) seien für den Fall der konformen Abbildung ebenfalls angeführt, da sie häufig zur Bildung von koordinatenfreien Differentialoperatoren benötigt werden, so sind sie beispielsweise an der kovarianten Differentiation 4.8 beteiligt. Setzt man abkürzend $\chi(x, y):=\xi_{x}^{2}+\eta_{x}^{2}$ und beachtet $f^{\prime \prime}(z)=\xi_{x x}+i \eta_{x x}$, so erhält man aus der Gleichheit der gemischten partiellen Ableitungen, den Cauchy-Riemannschen Differentialgleichungen und 4.6

$$
\begin{aligned}
& \Gamma_{11}^{1}=\Gamma_{12}^{2}=\Gamma_{21}^{2}=\frac{\xi_{x} \xi_{x x}+\eta_{x} \eta_{x x}}{\chi}, \quad \Gamma_{12}^{1}=\Gamma_{21}^{1}=\Gamma_{22}^{2}=\frac{\eta_{x} \xi_{x x}-\xi_{x} \eta_{x x}}{\chi}, \\
& \Gamma_{22}^{1}=-\Gamma_{11}^{1}=-\frac{\xi_{x} \xi_{x x}+\eta_{x} \eta_{x x}}{\chi}, \quad \Gamma_{11}^{2}=-\Gamma_{12}^{1}=\frac{\xi_{x} \eta_{x x}-\eta_{x} \xi_{x x}}{\chi} .
\end{aligned}
$$


Wird $f(z)=\zeta=\xi(x, y)+i \eta(x, y)$ schließlich durch Komposition zweier konformer Abbildungen $h(z)=w=u(x, y)+i v(x, y), h: \overline{\mathfrak{Q}} \rightarrow \overline{\mathfrak{E}}$, und $g(w)=\zeta=\xi(u, v)+i \eta(u, v), g: \overline{\mathfrak{E}} \rightarrow \overline{\mathfrak{G}}$, konstruiert, so muß zur Ermittlung der metrischen Größen bei Bildung der Ableitung die Kettenregel beachtet werden. Mit $f^{\prime}(z)=g^{\prime}(w) \cdot h^{\prime}(z)$ werden die Ableitungen mittels

$$
f^{\prime}(z)=\xi_{x}+i \eta_{x}=\left(\xi_{u}+i \eta_{u}\right) \cdot\left(u_{x}+i v_{x}\right)
$$

in jedem Rechengitterpunkt des Einheitsquadrates einmalig berechnet und extern abgespeichert. Für die zweiten Ableitungen von $f$ verfährt man in analoger Weise, es wird

$$
f^{\prime \prime}(z)=\xi_{x x}+i \eta_{x x}=\left(\xi_{u u}+i \eta_{u u}\right) \cdot\left(u_{x}+i v_{x}\right)^{2}+\left(\xi_{u}+i \eta_{u}\right) \cdot\left(u_{x x}+i v_{x x}\right) .
$$

\subsection{Randbedingungen}

Bei Kenntnis der konformen Abbildung und somit der metrischen Verhältnisse ist man bereits in der Lage, die im Inneren von $\mathfrak{G}$ geforderten, mittels Differentialoperatoren koordinatenfrei formulierten Gesetze in Verallgemeinerung der Vorgehensweise von Kapitel 1 einem Ortsdiskretisierungsprozeß zu unterwerfen, um über die CTDS-Linienmethode zu einem System gewöhnlicher Differentialgleichungen zu gelangen. Im Fall der Wärmeleitungsgleichung stellt es beispielsweise ein Leichtes dar, in den inneren Punkten des Rechengitters $\mathfrak{Q}_{n, n}$ den diskretisierten Laplace-Operator mit dem Faktor $1 / \chi\left(x_{i}, y_{j}\right)$ zu multiplizieren. Die Qualität der Simulation selbst muß bei einer solchen Vorgehensweise allerdings noch einer Überprüfung standhalten.

Gänzlich anders gelagert ist die Frage nach der Implementierung der Randbedingungen. Während bei der bisherigen Simulation am Einheitsquadrat die Verwendung von kartesischen Koordinaten gewissermaßen den kanonischen Zugang darstellt, steht man im Falle allgemeinerer Rechengebiete bei einer solchen Herangehensweise vor zwei Schwierigkeiten:

- eine äquidistante kartesische Parametrisierung trifft im allgemeinen Fall nicht sämtliche Punkte des Gebietsrandes, damit sind weder Dirichletbedingungen noch Bedingungen, bei denen ein funktionaler Zusammenhang der Randableitung vorgeschrieben wird, zufriedenstellend implementierbar. Dieser Sachverhalt wird in Abbildung 4.1 verdeutlicht.

- löst man das Problem der Randpunkte durch nicht äquidistante Diskretisierung (wodurch einerseits die Fehlerordnung in Mitleidenschaft gezogen wird, andererseits sich die Formeln erheblich verkomplizieren), so können Dirichletsche Randbedingungen implementiert werden. Bei Newtonschen Randbedingungen fällt der auswärts gerichtete Einheitsnormalenvektor allerdings nicht mehr mit der Richtung einer Koordinatenlinie zusammen, wodurch seine kartesischen Koordinaten im allgemeinen Fall von Randpunkt zu Randpunkt variieren werden.

Um die Natur der auswärts gerichteten Einheitsnormalenableitung eines auf einer Riemannschen Mannigfaltigkeit $\mathcal{R}$ gegebenen Skalarfeldes $\omega$ zu Tage zu fördern, betrachten wir dort ein kontravariantes Vektorfeld $\boldsymbol{v}$. Im Punkt $P \in \mathcal{R}$ gilt bezüglich zweier Karten die Darstellung

$$
\boldsymbol{v}(P)=v^{i} \boldsymbol{\partial}_{\boldsymbol{i}}=\bar{v}^{i} \overline{\boldsymbol{\partial}}_{\boldsymbol{i}}
$$

Dabei ist speziell zu beachten, daß für die Basisvektoren $\partial_{i}$ und $\bar{\partial}_{i}$ im allgemeinen Fall weder Orthogonalität noch Normiertheit gegeben sein muß. Über 4.1 ist die Invariante

$$
\nabla_{\boldsymbol{v}} \omega=d \omega(\boldsymbol{v})=\boldsymbol{v}(\omega)=v^{i} \frac{\partial(\omega \circ \kappa)}{\partial x_{i}}=\bar{v}^{i} \frac{\partial(\omega \circ \bar{\kappa})}{\partial \bar{x}_{i}}
$$

erklärt, durch die Richtungsableitung wird demnach bei Forderung nach entsprechenden Differenzierbarkeitseigenschaften einem Skalarfeld wieder ein Skalarfeld zugeordnet. 

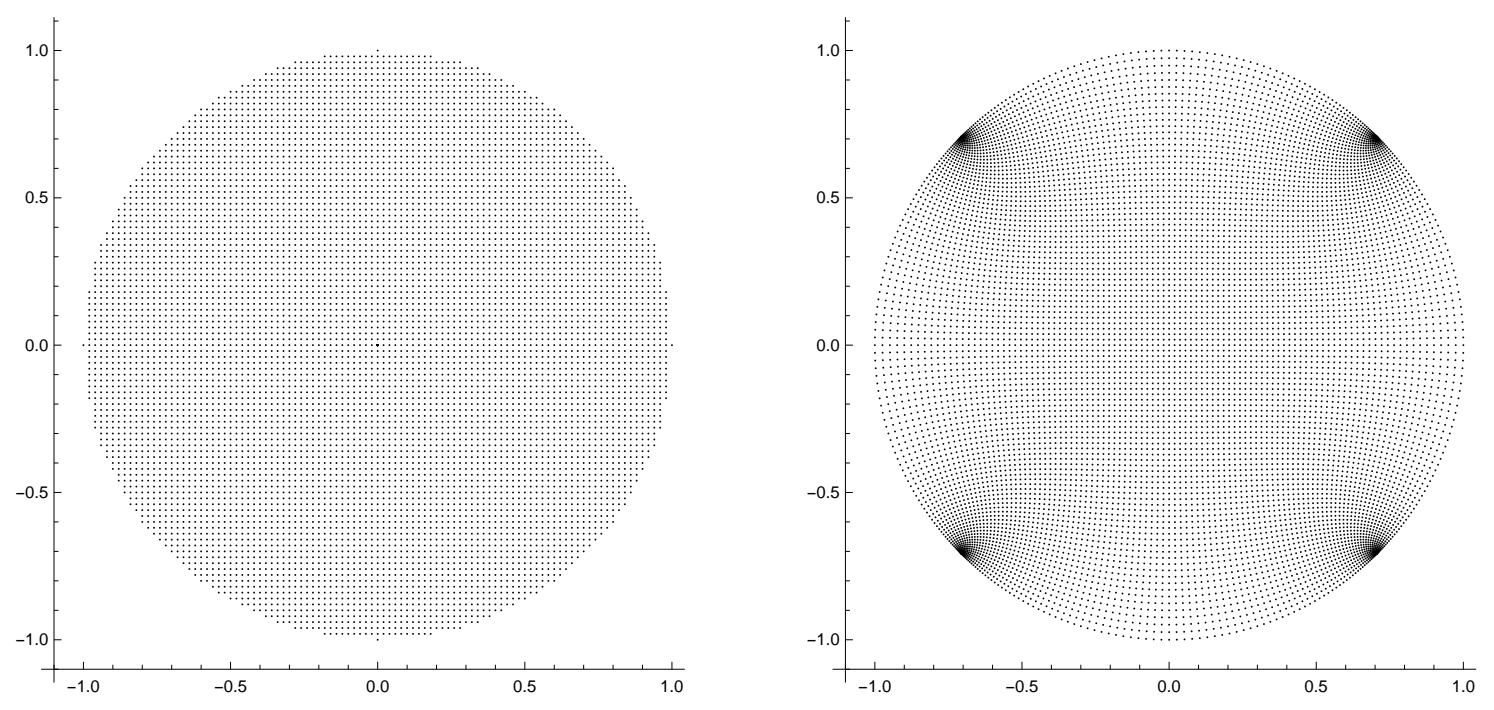

Abbildung 4.1: In kartesischen Koordinaten können bei äquidistantem Rechengitter am Einheitskreis die Randbedingungen nicht exakt implementiert werden, die Kreisperipherie enthält nur vier Gitterpunkte (links). Wird der Kreis durch das Quadrat parametrisiert, so entsprechen den Gitterpunkten auf den Quadratseiten jeweils Gitterpunkte auf der Kreisperipherie und Randbedingungen beliebiger Art können bequem einprogrammiert werden (rechts).

Für den Fall der konformen Abbildung erwächst nun die Problematik, daß — anders als bei der Transformation des in kartesischen Koordinaten konstanten Maßtensors - die kartesischen Koordinaten des Einheitsnormalenvektors bei Parametrisierung mittels der Karte $\kappa_{\zeta}$ von Punkt zu Punkt variieren und nicht explizit als Ausgangspunkt für eine Umrechnung gemäß 4.3 herangezogen werden können. Man kann aber den umgekehrten Weg beschreiten und versuchen, die Koordinaten des Einheitsnormalenvektors direkt in seinen neuen Koordinaten bezüglich der Karte $\kappa_{z}$ zu finden.

Betrachtet man dazu in der Karte $\kappa_{z}$ das Vektorfeld $\boldsymbol{v}(P)$ am Rande $\mathfrak{C}$ von $\mathfrak{G}$, so fallen die Koordinatenlinien laut Konstruktion der Abbildung jedenfalls mit $\mathfrak{C}$ zusammen, einer Quadratseite entspricht dabei jeweils einem Stück der Berandung. Exemplarisch gilt auf der Quadratunterseite für einen Normalenvektor somit die Darstellung $\boldsymbol{v}=-v^{y} \boldsymbol{\partial}_{\boldsymbol{y}}$, sodaß man über die Normierungsforderung

$$
\|\boldsymbol{v}\|=\sqrt{(\boldsymbol{v}, \boldsymbol{v})}=\sqrt{\left(-v^{y} \boldsymbol{\partial}_{\boldsymbol{y}},-v^{y} \boldsymbol{\partial}_{\boldsymbol{y}}\right)}=v^{y} \sqrt{g_{22}}=1
$$

übergehen kann zum nach außen gerichteten Einheitsnormalenvektor $\boldsymbol{n}=\frac{\boldsymbol{v}}{\|\boldsymbol{v}\|}$ mit den Koordinaten

$$
n^{x}=0, \quad n^{y}=-\frac{1}{\sqrt{g_{22}}} .
$$

Damit können in den Randpunkten des Quadratgitters Randbedingungen durch Diskretisierung analog zu 1.18)-1.21 implementiert werden, wie im Inneren versehen mit einem metrischen Korrekturfaktor. Man kann nun einwenden, daß der Maßtensor bzw. $f^{\prime}(z)$ in den Quadratecken verschwinden und eine Implementierung von Randbedingungen, die die Normalenableitung eines Skalarfeldes vorschreiben, durch die in den Ecken auftretende Singularität ja gar nicht möglich ist. Bei genauerer Betrachtung der Vorgehensweise in Kapitel 1 erkennt man aber, daß numerische Werte in den Eckpunkten zu keiner Zeit an der Simulation beteiligt sind und daher auch nicht bereitgestellt werden müssen. Diese für die Simulation essentiellen Erkenntnisse fassen wir zusammen im

Satz (Normalenableitung bei konformer Abbildung).

Die Ableitung eines Skalarfeldes in Richtung des nach außen gerichteten Einheitsnormalenvektors ist in den Koordinaten der Karte $\kappa_{z}$ zu bilden durch

$$
\nabla_{\boldsymbol{n}} \omega= \pm \frac{1}{\sqrt{\xi_{x}^{2}+\eta_{x}^{2}}} \frac{\partial \omega}{\partial x_{i}}
$$




\subsection{Wärmeleitung am Einheitskreis}

Es ist nun an der Zeit, diese reichlich theoretischen Ausführungen einer ernsthaften Überprüfung durch eine Simulationsstudie zu unterziehen. Ein weiteres Mal soll ein Problem, diesmal am Einheitskreis, formuliert werden, für das eine Reihenlösung zu Vergleichszwecken ermittelt werden kann und das somit dazu geeignet ist, globalen Fehler und Konvergenzordnung zu eruieren. Neben den beiden CTDS-Verfahren kommt auch wieder die PDE-Toolbox von Matlab zum Einsatz.

Gesucht sei als Reihenlösung die von der Zeit abhängige skalare Temperaturverteilung $u(\xi, \eta, t)$ auf dem offenen Einheitskreis $|\zeta|<1$ für beliebige Zeitpunkte $t \in(0, \infty)$, auf dessen Innerem die instationäre Wärmeleitungsgleichung gelte, diesmal mit ortsabhängiger Anfangsverteilung:

$$
\frac{\partial u}{\partial t}=\kappa \Delta u, \quad u(\xi, \eta, 0)=1-\left(\xi^{2}+\eta^{2}\right), \quad|\zeta|<1 \quad \forall \zeta=\xi+i \eta .
$$

Am Rand des Einheitskreises fordern wir für $t>0$

$$
u(\xi, \eta, t)=0, \quad|\zeta|=1 \quad \forall \zeta=\xi+i \eta .
$$

Zur Lösung kann man sich an den Ausführungen von Abschnitt 1.2 orientieren, Einführung von Polarkoordinaten erbringt eine nur von $r$ und $t$ abhängige Darstellung mittels BEsseLscher Funktionen $^{4}$ erster Gattung ([7, S.1227, (37.62)] und [5, S.207]) mit den Nullstellen $\lambda_{0, k}$ von $\mathfrak{J}_{0}(x)$,

$$
u(\xi, \eta, t)=u\left(\sqrt{\xi^{2}+\eta^{2}}, t\right)=u(r, t)=4 \sum_{k=1}^{\infty} \frac{e^{-\kappa t \lambda_{0, k}^{2}} \mathfrak{J}_{0}\left(r \lambda_{0, k}\right) \mathfrak{J}_{2}\left(\lambda_{0, k}\right)}{\lambda_{0, k}^{2}\left(\mathfrak{J}_{0}\left(\lambda_{0, k}\right)^{2}+\mathfrak{J}_{1}\left(\lambda_{0, k}\right)^{2}\right)} .
$$

Nähere Erläuterungen zum Lösungsweg in [7, S.1161ff., Bsp. 36.15] und [18]. Die Lösung kann man sich in diesem Fall sogar auch durch Mathematica symbolisch bestätigen lassen ${ }^{5}$.
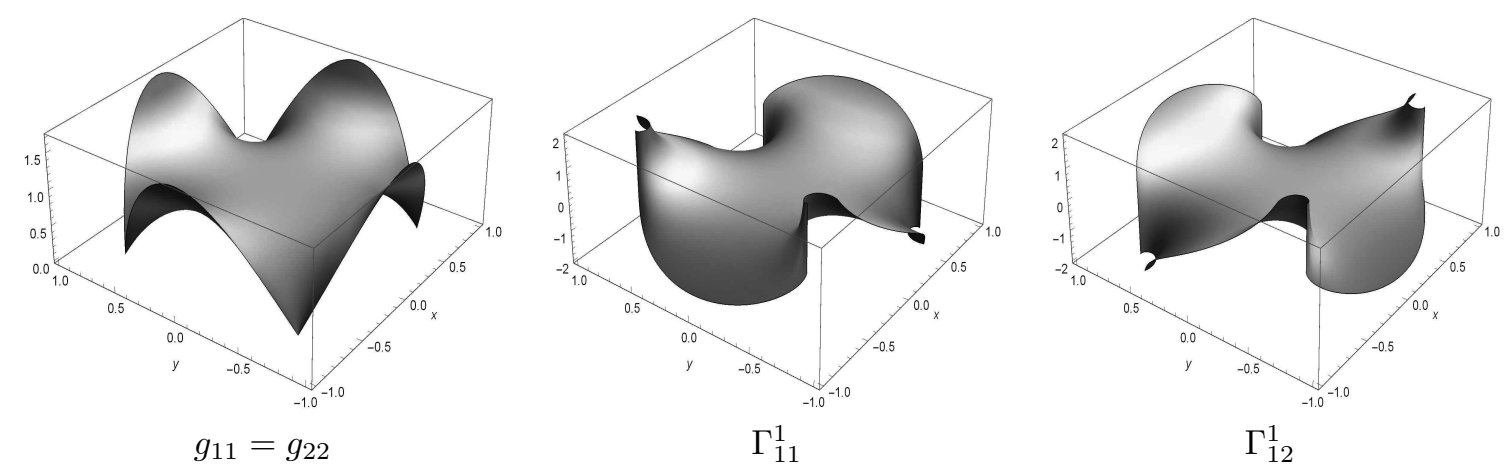

Abbildung 4.2: Der Maßtensor und zwei der Zusammenhangskoeffizienten 4.11 auf $\overline{\mathfrak{E}}$.

Da im Zuge der Implementierung die Temperaturwerte in den Randgitterpunkten in diesem Fall auf Grund des Vorliegens einer Dirichlet-Randbedingung fix vorgeschrieben werden können, betrifft die einzige weitere Modifikation im Programmcode den Laplace-Operator, dessen diskretisierte Form durch den Wert des kovarianten Maßtensors im jeweiligen Gitterpunkt zu dividieren ist. Das so erhaltene System gewöhnlicher Differentialgleichungen kann wieder von Mathematica mittels NDSolve aufintegriert werden. Durch den Umstand, daß der Maßtensor nun von Punkt zu Punkt variiert, wäre intuitiv eine Auswirkung auf den Diskretisierungsfehler zu erwarten. Wie die nachfolgenden Ergebnisse verdeutlichen, ist dem erstaunlicherweise nicht so. Tabelle 4.1 zeigt, daß neben der hohen Genauigkeit (FD2-Verfahren) bei Verwendung einer genügend großen Anzahl an Diskretisierungspunkten sogar die Konvergenzordnung erhalten bleibt. Die Gitter wurden dabei dergestalt dimensioniert, daß ein direkter Vergleich mit der PDE-Toolbox möglich wird.

\footnotetext{
${ }^{4}$ Die Besselschen Funktionen $\mathfrak{J}_{n}(z)=\frac{1}{\pi} \int_{0}^{\pi} \cos (z \sin \varphi-n \varphi) \mathrm{d} \varphi$ genügen $(n \in \mathbb{Z})$ dabei der Besselschen Diffentialgleichung $x^{2} y^{\prime \prime}+x y^{\prime}+\left(x^{2}-n^{2}\right) y=0$.

${ }^{5}$ eqn $=r D[u[r, t], t, 1]==\kappa D[r D[u[r, t], r], r] ; b c=u[1, t]==0 ; i c=u[r, 0]==1-r^{2}$; dsol=DSolve $[\{$ eqn, bc,$i c\}, u[r, t],\{r, t\}]$
} 


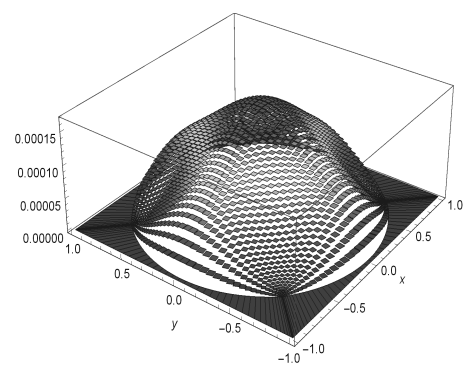

FD1 2.116 Pt

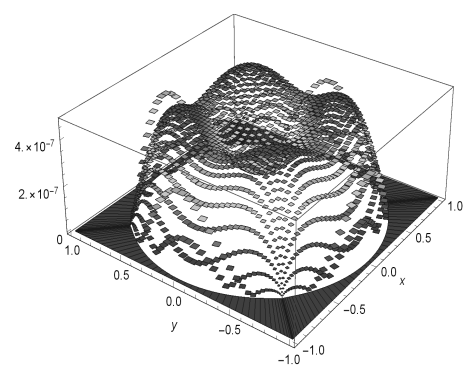

FD2 2.116 Pt

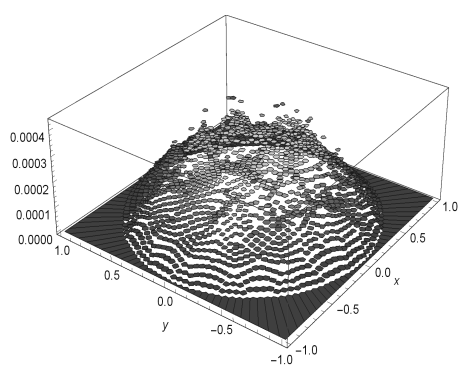

PdeTbx $2.129 \mathrm{Pt}$

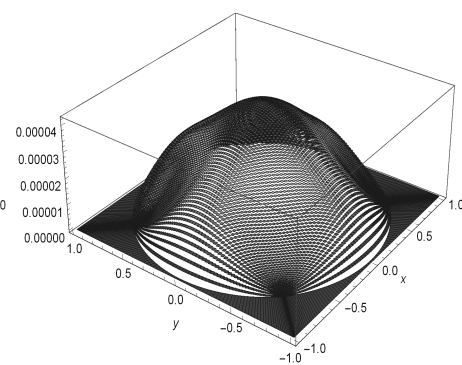

FD1 $8.281 \mathrm{Pt}$

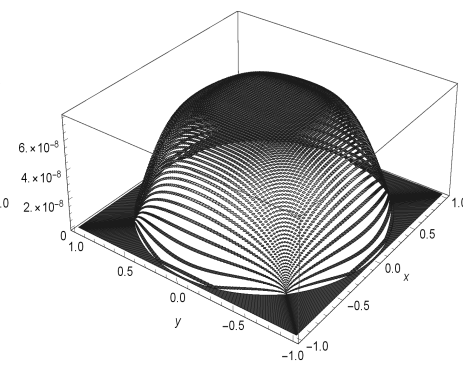

FD2 $8.281 \mathrm{Pt}$

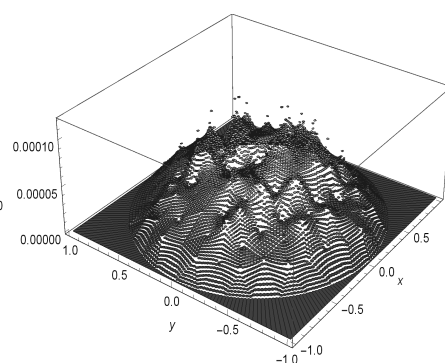

PdeTbx $8.385 \mathrm{Pt}$

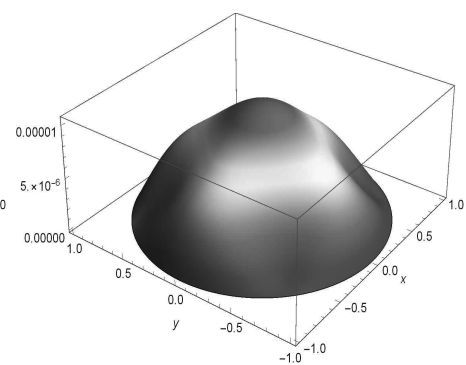

FD1 $32.761 \mathrm{Pt}$

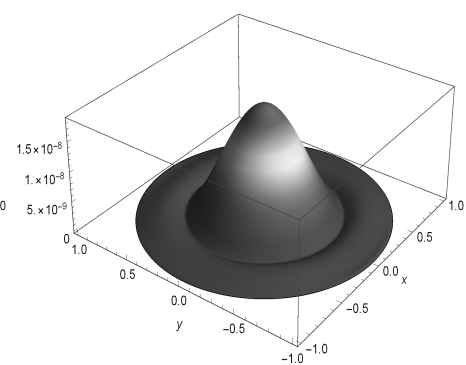

FD2 $32.761 \mathrm{Pt}$

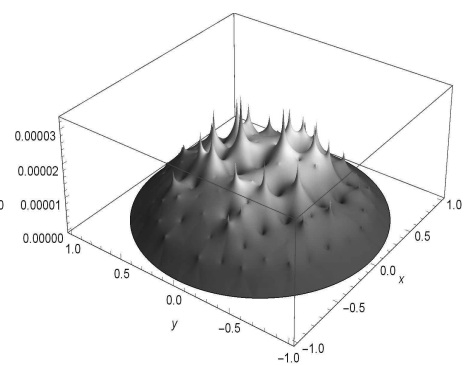

PdeTbx 33.281 Pt

Abbildung 4.3: Simulationslösung von Problem 4.13-4.14): Vergleich der absoluten Fehler.

\begin{tabular}{|c||c|c|c|}
\hline \hline Verfahren & Anzahl Gitterpunkte & Absolutes Fehlermittel & errechnete Konvergenzordnung \\
\hline \hline$F D 1$ & 2.116 & $6.27281 * 10^{-5}$ & - \\
\hline$F D 1$ & 8.281 & $1.61182 * 10^{-5}$ & 3.89176 \\
\hline$F D 1$ & 32.761 & $4.08124 * 10^{-6}$ & 3.94933 \\
\hline$F D 2$ & 2.116 & $2.58897 * 10^{-7}$ & - \\
\hline$F D 2$ & 8.281 & $4.55414 * 10^{-8}$ & 5.68487 \\
\hline$F D 2$ & 32.761 & $2.83388 * 10^{-9}$ & 16.07035 \\
\hline PdeTbx & 2.129 & $1.48329 * 10^{-4}$ & - \\
\hline PdeTbx & 8.385 & $3.76648 * 10^{-5}$ & 3.93812 \\
\hline PdeTbx & 33.281 & $9.48946 * 10^{-6}$ & 3.96912 \\
\hline
\end{tabular}

Tabelle 4.1: Vergleich der arithmetischen Mittel des absoluten Fehlers bei Simulationslösung der instationären Wärmeleitungsgleichung (4.13) am Einheitskreis. Wegen der Dirichlet-Randbedingung entfallen 1.14-1.17 und die Konvergenzordnung von FD1 wird sogar quadratisch. 


\subsection{Quadratwärmeleitung revisited (experimentum crucis)}

Nach den überraschend exakt ausgefallenen Ergebnissen der Simulation am Einheitskreis vom vorhergehenden Abschnitt soll versucht werden, die Simulation aus Kapitel 1 unter den Voraussetzungen zu wiederholen, daß nun das Quadrat durch sich selbst über den Einheitskreis konform parametrisiert wird. In der Praxis stellt diese Vorgehensweise natürlich den denkbar ungünstigsten Zugang zur Problematik dar, sie rechtfertigt sich jedoch neben den Vergleichsmöglichkeiten zur analytischen Lösung auch als Teststellung, die zur Prüfung der Korrektheit der Implementierung trefflich geeignet ist. Als Ausgangspunkt dient die in Abschnitt 3.3 numerisch berechnete und extern abgespeicherte Ränderzuordnungsfunktion $\theta(\varphi)$, die den Zusammenhang des Winkels am Kreis mit dem Winkel am Quadrat mittels einer kontinuierlichen Interpolierenden mit speziellen Basisfunktionen herstellt ${ }^{6}$. Die exakten konformen Abbildungen (und deren Ableitungen) liegen zwar in Form der Schwarz-Christoffel-Transformation und deren Umkehrung vor, aber diese Informationen sollen nur zur Abbildung des Rechengitters auf den Kreis und zu Vergleichszwecken verwendet werden. Die numerische Abbildung des auf den Kreis transformierten Rechengitters sowie die Berechnung der dortigen Ableitungsgrößen soll ausschließlich mit Kenntnis von $\theta(\varphi)$ und $\rho(\theta)$ erfolgen.

Wir untersuchen zunächst die Verhältnisse an der Berandung und wenden uns dann dem Inneren des Einheitskreises zu, $z, w$ und $\zeta$ mögen dabei die unabhängigen Variablen am Rechengitter, Kreis und dem Quadrat als dem Bildgebiet bezeichnen. Der Rand des Quadrates wurde durch die Secans-Funktion parametrisiert ${ }^{7}$ und bei der Herleitung der THEODORsENschen Integralgleichung wurde zur Ermittlung der Ränderzuordnungsfunktion, ausgehend von $f$, die Hilfsfunktion

$$
F(w)=\ln \frac{f(w)}{w}=\ln |f(w)|-\ln |w|+i \arg f(w)-i \arg w
$$

betrachtet, sodaß es keine Schwierigkeiten bereitet, die Bilder der Kreisperipherie sofort durch

$$
f\left(e^{i \varphi}\right)=\rho(\theta(\varphi)) \cdot e^{i \theta(\varphi)}
$$

mit alleiniger Kenntnis von $\theta(\varphi)$ für beliebige Punkte $e^{i \varphi}$ am Kreisrand berechnen zu lassen. Da bei der vorliegenden Problemstellung keine Randbedingungen vom Dirichlet-Typ vorgeschrieben sind, müssen zur Auflösung der Newtonschen Randbedingungen die metrischen Größen auch am Rand des Rechengitters zur Verfügung gestellt werden und nach kurzem Rechengang findet man

$$
f^{\prime}\left(e^{i \varphi}\right)=\theta^{\prime}(\varphi) \cdot e^{i(\theta(\varphi)-\varphi)} \cdot\left[\rho(\theta(\varphi))-i \rho^{\prime}(\theta(\varphi))\right]
$$

was mit Sicherheit einen der schnellsten Wege darstellt, um numerisch an die am Kreisrand benötigten Ableitungsgrößen zu gelangen. Für Ableitungen höherer Ordnung kann man analog nach der Kettenregel vorgehen, diese werden aber (so sie überhaupt existieren) zur Implementierung von Randbedingungen im allgemeinen nicht benötigt. Im übrigen ist auch die Existenz der ersten Ableitung an der Kreisperipherie noch zu untermauern, $f$ ist dort jedenfalls stetig und mit der Rektifizierbarkeit des Quadratrandes folgt über die absolute Stetigkeit, daß $f$ fast überall (also mit Ausnahme der Urbilder, die den Quadratecken entsprechen) differenzierbar ist [27, S.70, S.123] ${ }^{8}$.

Hinsichtlich der Abbildung der Punkte aus dem Inneren des Einheitskreises kann Mathematica mit der SchwARZschen Integralformel 2.17 gut umgehen ${ }^{9}$, das Kurvenintegral wird besonders zügig ausgewertet, wenn der Integrationsroutine die Lage der Eckwinkel bekanntgegeben wird.

Zur Vollständigkeit der metrischen Größen fehlen noch die Ableitungen im Inneren des Einheitskreises, und dazu geht man folgendermaßen vor. Wir greifen die in Abschnitt 2.2 anlaßbezogen verschobene Diskussion hinsichtlich der Integraldarstellung der $k$-ten Ableitungen einer analytischen Funktion wieder auf und finden mit [17, S.246, Theorem 4.7d] folgenden

\footnotetext{
${ }^{6}$ verwendet wird die genauere der dort vorgestellten Ränderzuordnungsfunktionen.

7 rho $\left[\mathrm{x}_{-}\right]=\mathrm{Sec}[\operatorname{Mod}[\mathrm{x}+\mathrm{Pi} / 4, \mathrm{Pi} / 2]-\mathrm{Pi} / 4]$;

${ }^{8}$ Priwalow verwendet den Satz von F. U. M. Riesz, die absolute Stetigkeit folgt, wenn $f$ am Kreisrand stetig und von endlicher Variation ist. Rektifizierbare Jordankurven sind von endlicher Variation.

${ }^{9} \mathrm{f}\left[\mathrm{w}_{-}\right]:=\mathrm{w} * \operatorname{Exp}\left[\frac{1}{2 \pi} * \mathrm{NIntegrate}\left[\log [\mathrm{r}[\operatorname{theta}[\mathrm{y}]]] *((\operatorname{Exp}[\mathrm{I} * \mathrm{y}]+\mathrm{w}) /(\operatorname{Exp}[\mathrm{I} * \mathrm{y}]-\mathrm{w})),\left\{\mathrm{y}, 0, \frac{\pi}{4}, \frac{3 \pi}{4}, \frac{5 \pi}{4}, \frac{7 \pi}{4}, 2 \pi\right\}\right]\right]$
} 
Satz (Darstellung von $f^{(k)}$ mittels verallgemeinerten CAUCHYschen Integralen).

Der Rand $\mathfrak{C}$ sei gegeben durch eine positiv orientierte Jordan-Kurve $\gamma$ mit Innerem $\mathfrak{G}$ dergestalt, daß $\mathfrak{C} \cup \mathfrak{G}$ dem Einheitskreis stückweise diffeomorph ist. Ist $f(z)$ auf $\mathfrak{G}$ regulär und auf $\mathfrak{C} \cup \mathfrak{G}$ stetig, dann gilt $\forall z \in \mathfrak{G}$

$$
f^{(k)}(z)=\frac{k !}{2 \pi i} \oint_{\gamma} \frac{f(\zeta)}{(\zeta-z)^{k+1}} \mathrm{~d} \zeta .
$$

Nicht nur die holomorphe Funktion selbst, sondern auch deren Ableitungen sind somit im Inneren allein durch die Funktionswerte am Rand bestimmbar. Diese Aussage läßt sich auf die Hilfsfunktion $F(w)$ aus 4.15 anwenden, mit $\zeta=e^{i \vartheta}$ und $\mathrm{d} e^{i \vartheta}=i e^{i \vartheta} \mathrm{d} \vartheta$ folgt über

$$
F^{\prime}(w)=\frac{f^{\prime}(w)}{f(w)}-\frac{1}{w}=\frac{1}{2 \pi} \int_{0}^{2 \pi} \frac{\ln \left|f\left(e^{i \vartheta}\right)\right|-\ln \left|e^{i \vartheta}\right|+i\left(\arg f\left(e^{i \vartheta}\right)-\arg e^{i \vartheta}\right)}{\left(e^{i \vartheta}-w\right)^{2}} e^{i \vartheta} \mathrm{d} \vartheta
$$

die gesuchte Darstellung

$$
f^{\prime}(w)=\frac{f(w)}{w}+\frac{f(w)}{2 \pi} \int_{0}^{2 \pi} \frac{\ln \rho(\theta(\vartheta))+i(\theta(\vartheta)-\vartheta)}{\left(e^{i \vartheta}-w\right)^{2}} e^{i \vartheta} \mathrm{d} \vartheta
$$

Der Ursprung $w=0$ macht wegen der unbestimmten Form dabei noch Kopfzerbrechen. In diesem Fall liefert der zweite Summand in (4.17) keinen Beitrag, da nach Voraussetzung $f(0)=0$ und $f^{\prime}(0)>0$ gilt. Eine erneute Anwendung der CAUCHYschen Integralformel, diesmal auf die Funktion $f(w) / w$, liefert auch den dortigen Wert der Ableitung $\left(\approx 1.0787052\right.$, Fehler $\left.2.25 \cdot 10^{-15}\right)$ durch

$$
f^{\prime}(0)=\frac{1}{2 i \pi} \oint_{\gamma} \frac{f(\zeta)}{\zeta(\zeta-0)} \mathrm{d} \zeta=\frac{1}{2 \pi} \int_{0}^{2 \pi} \frac{\rho(\theta(\vartheta)) e^{i \theta(\vartheta)}}{e^{i \vartheta}} \mathrm{d} \vartheta
$$

Mit den obigen Formeln ist man in der Lage, die Punktabbildung $\overline{\mathfrak{Q}} \rightarrow \overline{\mathfrak{E}} \rightarrow \overline{\mathfrak{Q}}$ sowie sämtliche zur Simulation notwendigen metrischen Größen durch Mathematica berechnen zu lassen. Die nötigen Fallunterscheidungen implementiert man mit der Routine Piecewise [], die numerischen Werte am Rechengitter werden nur einmalig berechnet und zur späteren Verwendung extern abgespeichert. Abbildung 4.4 visualisiert die Fehlerverhältnisse, wenn $e(z):=|\zeta-z|$ und $e^{\prime}(z):=\left|\zeta^{\prime}-1\right|$ betrachtet wird $^{10}$. Wir bemerken noch, daß eine Verfeinerung des Rechengitters auf $e(z)$ keine Auswirkungen hat, für $e^{\prime}(z)$ würde man dies auf Grund der dichteren Lage der Bildpunkte am Einheitskreis um $\frac{k \pi}{4}$ herum intuitiv erwarten, da die Güte der Approximation von $\theta^{\prime}$ bei Annäherung an die Quadratecken gemäß Abbildung 3.5 abnimmt. Für die betrachteten Rechengitter zeigen sich aber diesbezüglich keine Effekte, weder qualitativ noch quantitativ. Ferner werden die Fehlermaxima jeweils wieder am Rand angenommen.

Die hergeleiteten Formeln haben nicht nur für den konkret betrachteten Fall der Abbildung des Einheitskreises auf das Quadrat Gültigkeit, sondern sind in allen Fällen anwendbar, in denen die Ränderzuordnung bereitgestellt werden kann. Man sieht aber, daß dafür einiger theoretischer Aufwand getrieben werden muß, der sich zusätzlich steigert, falls die Gestalt der in einer betrachteten Anfangsrandwertaufgabe involvierten Differentialoperatoren es notwendig macht, auch Ableitungen höherer Ordnung zu studieren. Wir bemerken, daß diese Anstrengungen bei entsprechenden Glattheitseigenschaften der Berandung und hinreichender Kreisnähe des Gebietes durch Verwendung trigonometrischer Interpolation zur Konstruktion der Ränderzuordnung vermieden werden können, denn dann sind mit $f(w)=w e^{P(w)}$ auch die Ableitungen viel bequemer auswertbar.

In Kapitel 1 wurde der Simulationslösung die exakte Lösung zum Vergleichszeitpunkt $t=4$ gegenübergestellt und das absolute Fehlermittel am Quadratgitter betrachtet, was auch für diesen Abschnitt gelten soll. Bei Auswertung der Reihenlösung 1.11 taucht zunächst die Fragestellung auf, ob die Variable $z$ oder $\zeta$ als Argument zur Auswertung herangezogen werden soll. Durch die

\footnotetext{
${ }^{10}$ Bei Bildung der Ableitung kommt $\sqrt{4.12}$ zur Anwendung, die Singularitäten in den Quadratecken können unberücksichtigt bleiben, da bei der Simulation keine Werte an diesen Stellen benötigt werden.
} 

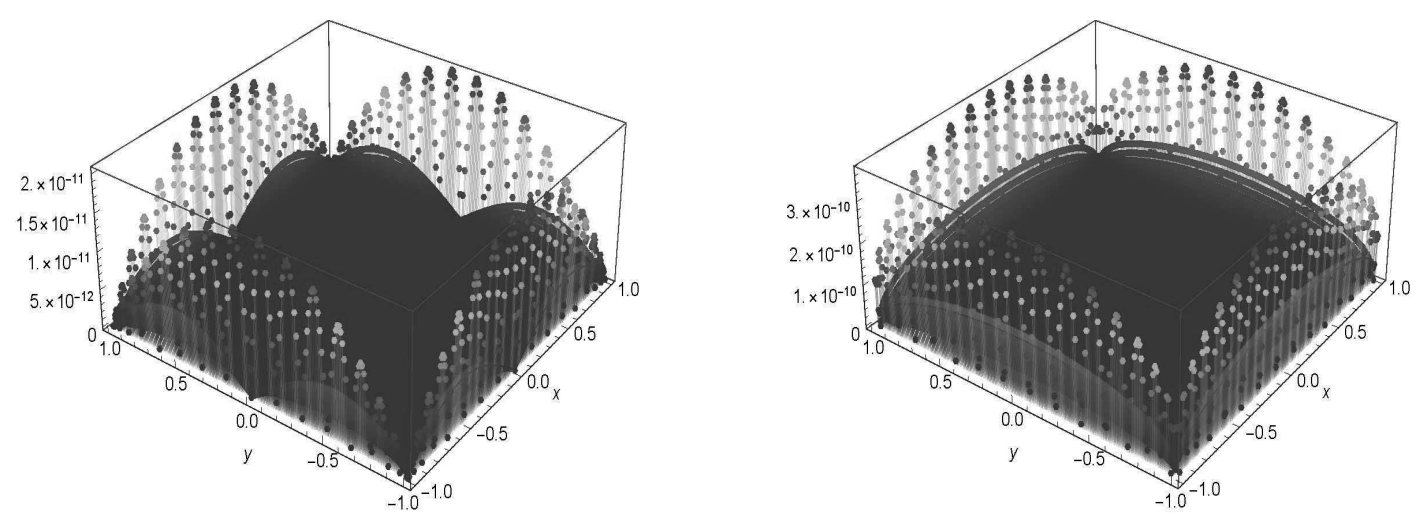

Abbildung 4.4: $e(z)$ und $e^{\prime}(z)$ auf $\mathfrak{Q}_{201,201}$ bei Parametrisierung des Quadrates durch sich selbst.

konforme Abbildung entsteht nämlich eine geringfügige numerische Verschiebung der Gitterpunkte in der (maximalen) Größenordnung $e(z) \approx 2.10^{-11}$, wobei davon wieder die Randpunkte am stärksten betroffen sind. Die Frage kann dahingehend beantwortet werden, daß für die Menge der Punkte des Rechengitters als Teilmenge einer Mannigfaltigkeit eine Punkttransformation nicht in Frage kommt. Vielmehr wird durch die konforme Abbildung eine Koordinatentransformation vermittelt und folglich ist die Reihenlösung mit $z$ als Argument auszuwerten. Zur Visualisierung des Temperaturfeldes sind dann natürlich die kartesischen Koordinaten $(\xi, \eta)$ zu verwenden.

Die Modifikation des in Kapitel 1 verwendeten Simulationscodes gestaltet sich überaus einfach, nach Einlesen der bereits berechneten und abgespeicherten (komplexen) Zahlenwerte für $f^{\prime}(i, j)$ bildet man in den Gitterpunkten die Größe $\chi(i, j)=\left|f^{\prime}(i, j)\right|^{2}$, durch die der diskretisierte LaplaceOperator zu dividieren ist. Die Randbedingungen an den Gitterseiten bleiben bis auf die Unterseite unverändert, dort ist bei Verwendung der 5-Punkt-Diskretisierung (1.21) zu ersetzen durch

$$
u(i, 1)=\frac{48 u(i, 2)-36 u(i, 3)+16 u(i, 4)-3 u(i, 5)}{25+12 \sqrt{\chi(i, 1)} \Delta y} .
$$

Es stellt sich erfreulicherweise heraus, daß die über die Metrik in die Wärmeleitungsgleichung eingebrachte Unschärfe im Standardfall nicht ausreichend ist, um wesentlichen Einfluß auf das Ergebnis auszuüben. Der relative Fehler deckt sich für $51 \times 51$ und $101 \times 101$ Gitterpunkte qualitativ wie quantitativ mit den Resultaten aus Kapitel 1, lediglich an der Unterseite des Quadrates sind bei Verfeinerung des Rechengitters auf $201 \times 201$ Punkte im relativen Fehler erste minimale Oszillationen feststellbar. Daraus kann bei der vorliegenden Konstellation geschlossen werden, daß die numerischen Ungenauigkeiten von $\chi$ erst dann beginnen Wirkung zu zeigen, wenn man die Simulationslösung in den Genauigkeitsbereich von 10 Nachkommastellen, mithin in den Bereich der Approximationsgüte der Ränderzuordnungsfunktion selbst, treibt. Bei eventuell auftretenden Problemen wäre demnach an eine Verbesserung der Güte der Ränderzuordnungsfunktion zu denken. Der Vollständigkeit halber sind die berechneten absoluten Fehlermittel in Tabelle (1.1) angeführt. 


\section{Kapitel 5}

\section{Studie: Wärmeleitung im Zahn}

In der Zahnmedizin verwendet man Lasertechnologien zur Behandlung von Entzündungsherden an der Wurzelspitze als weitgehend schmerzfreie Alternative zur Resektion [9]. Letztere stellt einerseits einen unangenehmen chirurgischen Eingriff dar, andererseits kommt es nach einiger Zeit dennoch häufig zur erneuten Abszessbildung und in weiterer Folge zum Verlust des Zahnes. Es wird daher versucht, den befallenen Bereich über den Zahnkanal zu erreichen und durch Wärmebehandlung mittels Laser eine Abheilung der Entzündung zu erzielen, wobei als wesentliche Nebenbedingung bei dieser Behandlungsmethode eine Schädigung des angrenzenden, gesunden Gewebematerials durch Überhitzung unter allen Umständen zu vermeiden ist. Wir entwickeln in diesem abschließenden Kapitel ein dreidimensionales Simulationsmodell unter Zuhilfenahme der konformen Abbildung, das Aufschluß über die Wärmeverteilung im Zahn bei Laserbehandlung ermöglichen soll.

\subsection{Anatomische Grundlagen}

Abbildung 5.1 gibt einen schematischen Überblick über einen im Kiefer verankerten Zahn ${ }^{1}$ und zeigt ein Röntgenbild eines Entzündungsherdes ${ }^{2}$. Man unterscheidet zunächst zwischen Zahnkrone (8), also dem sichtbaren Bereich, und der Zahnwurzel (12), die Grenze (11) wird als Zahnhals bezeichnet.

Im Innersten des Zahnes, der Pulpa (4,5,6 und 25), befindet sich nerven- und gefäßreiches Bindegewebe, das über einen Eintritt an der Wurzelspitze (15) versorgt wird und sensorisch an den fünften Gehirnnerv (N. trigeminus) angebunden ist. Die Pulpa wird zur Gänze umgeben vom Zahnbein oder Dentin (3), einer knochenähnlichen Substanz, die von mikroskopischen Kanälen durchsetzt ist, in denen radiär die Nervenbahnen auslaufen. Der Großteil der gesamten Zahnsubstanz besteht aus Zahnbein. Das empfindliche Dentin ist im Bereich der Zahnkrone mit einer emailähnlichen Schutzschicht überzogen, dem Zahnschmelz oder Enamel (2), der härtesten Substanz des Körpers.

Im Bereich des Zahnhalses geht der Zahnschmelz über in das Cementum (7/grün dargestellt), den Zahnzement, das die gesamte Wurzel in einer dünnen Schicht überzieht. Hier findet auch der Übergang vom Zahn- zum Zahnhalteapparat oder Parodont (17) statt, der den Zahn mit dem umliegenden Knochengewebe (23) fest verankert. Der Bereich des Parodontiums wird durch hohe Temperaturen geschädigt, was bei Einbringung einer Wärmequelle über den Wurzelkanal in den Bereich des Entzündungsherdes zu berücksichtigen ist. In Analogie des Überganges von Enamel auf Cementum erfolgt in einer weiteren Gewebsschicht des Halteapparates ein Übergang des Zahnfleisches, Gingiva (19), auf die Wurzelhaut oder Desmodont (22). In diesem thermisch sehr sensiblen Bereich erfolgt unter anderem die Neubildung von Fibroblasten, Fibrillen, Cementum und Knochengewebe.

\footnotetext{
${ }^{1}$ https://commons.wikimedia.org/wiki/File:Cross sections_of teeth_intl.svg

${ }^{2}$ Mit freundlicher Genehmigung nach Anfrage per Email an DR. MARTIN S. SPILler, https://doctorspiller.com
} 

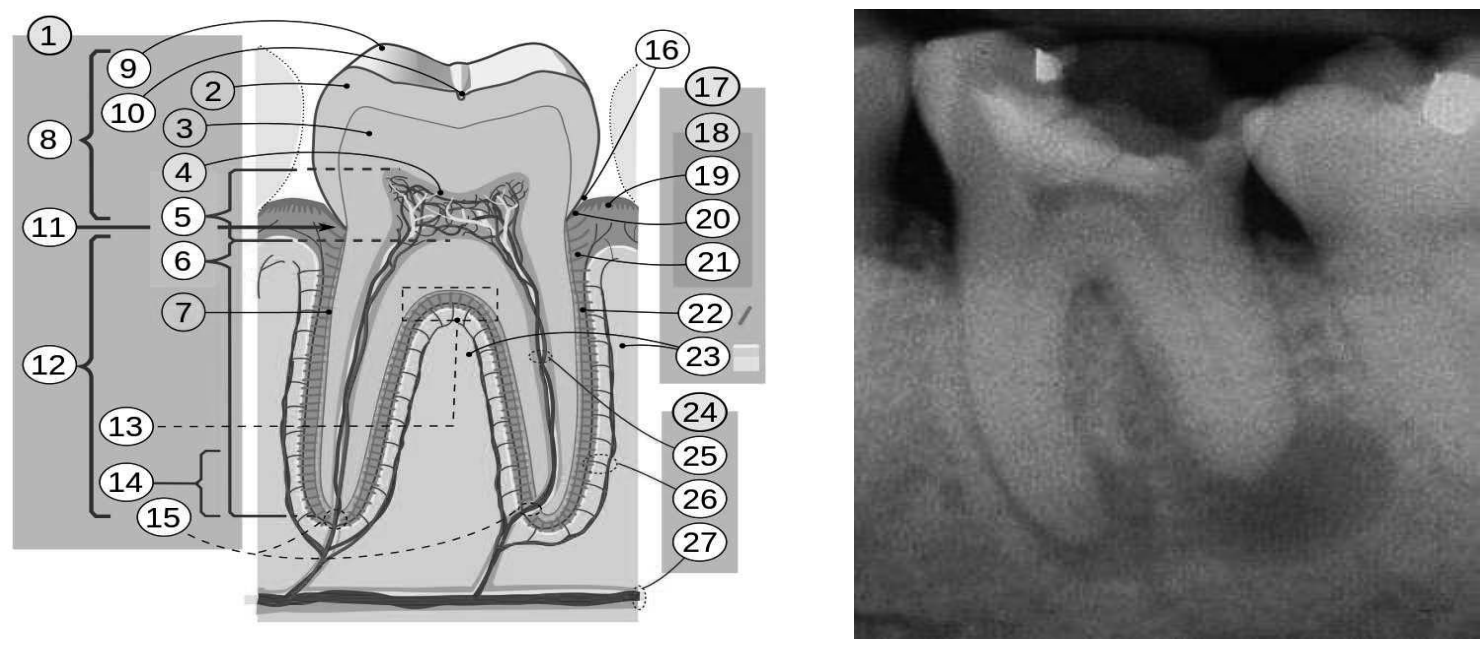

Abbildung 5.1: Klassifizierung der Zahnbereiche durch schematische Darstellung des Zahnquerschnitts (links) und Entzündungsherde an der Wurzelspitze am Röntgen (rechts).

\subsection{Entwicklung des Grundmodells}

Wählt man als zweidimensionales Simulationsgebiet eines der in Abbildung 3.7 durch das Einheitsquadrat parametrisierten Gebiete, wobei den rein imaginären Punkten auf der $\eta$-Achse die Öffnung des Zahnkanals (über den später eine Wärmequelle eingebracht werden soll) entsprechen möge und an der Berandung des Gebietes der Bereich des Parodonts bzw. die Zahnkrone angesiedelt ist, so fehlen zur Durchführung eines Simulationslaufes noch die Ableitungen der zugehörigen konformen Abbildung, mit deren Hilfe dann der metrische Fundamentaltensor und andere relevante Größen wie die Christoffelschen Symbole ermittelt werden können. Im Falle der Simulation am Einheitskreis waren diese Größen analytisch bekannt bzw. wurde deren Herleitung für den Fall der numerischen Quadratabbildung über den Einheitskreis in Abschnitt 4.6 eingehend erörtert.

Zur numerischen Ermittlung der metrischen Größen der Abbildung $f=g \circ h: \overline{\mathfrak{Q}} \rightarrow \overline{\mathfrak{G}}$ vom Quadrat auf den Zahnquerschnitt ist zu beachten, daß in diesem Fall die konforme Abbildung vom Einheitskreis auf den Querschnitt, $g: \overline{\mathfrak{E}} \rightarrow \overline{\mathfrak{G}}$, über das konjugierte trigonometrische Interpolationspolynom $P(w)$ konstruiert wurde. Wählt man daher für $h(z), h: \overline{\mathfrak{Q}} \rightarrow \overline{\mathfrak{E}}$, die Transformation (2.7), bildet mit $g(w)=w \cdot e^{P(w)}$ und $P(w)=a_{0} / 2+\sum\left(a_{k}-i b_{k}\right) w^{k}$ nach der Kettenregel $\sqrt{4.12} g(h(z))^{\prime}$, so errechnen sich die Ableitungen der konformen Abbildung vom Quadrat auf den Querschnitt zu

$$
\begin{aligned}
& f^{\prime}(z)=\xi_{x}+i \eta_{x}=g^{\prime}(w) h^{\prime}(z)=h^{\prime}(z) e^{P(w)}\left(w P^{\prime}(w)+1\right), \\
& f^{\prime \prime}(z)=\xi_{x x}+i \eta_{x x}=g^{\prime \prime} h^{\prime 2}+h^{\prime \prime} g^{\prime}=e^{P}\left[h^{\prime \prime}+P^{\prime}\left(2 h^{\prime 2}+w h^{\prime \prime}+w h^{\prime 2} P^{\prime}\right)+w h^{\prime 2} P^{\prime \prime}\right] .
\end{aligned}
$$

Da die Fourier-Koeffizienten bereits im Zuge der Iteration der Ränderzuordnungsfunktion berechnet wurden und extern abgespeichert sind, können diese Ausdrücke mit Mathematica bequem implementiert, einmalig berechnet und für verschiedene Gittergrößen ebenfalls extern abgespeichert werden. Um auch hier wieder Fehlerquellen bei der Implementierung auszuschließen bietet es sich an, die Ableitungen unter Verwendung des trigonometrischen Interpolationspolynoms für den Fall der gespiegelten Ellipse testweise auszuwerten und mit jenen der exakten Abbildung (3.2) abzugleichen. Für jede der drei Geometrien kann somit eine Liste mit sämtlichen für das diskrete Gitter relevanten Informationen $^{3}$ ausgewählt werden, die vor dem Simulationslauf eingelesen wird.

\footnotetext{
${ }^{3}$ Abgespeichert werden die Gitterdaten in Form einer Liste komplexer $n \times n$-Matrizen: gitterinfo=Chop [\{zgrid, wgridexakt, zeta,fstrichgrid,f2strichgrid,nprec $\}, 10^{`}$-nprec]
} 
Als erste Testsimulation wurde auf den drei Zahngeometrien wieder die instationäre Wärmeleitungsgleichung mit Anfangsbedingung $u(\xi, \eta, 0)=1$ gerechnet, als Randbedingung sei auf dem der Quadratoberseite entsprechenden Teil der Berandung $\nabla_{\mathbf{n}} u=-u$ vorgeschrieben, die restlichen drei Quadratseiten mögen adiabatisch isoliert angenommen werden. Dabei kann zur Diskretisierung der Quadratoberseite wieder 4.18 verwendet werden. Die resultierenden Temperaturverteilungen sind in Abbildung 5.2 ersichtlich, der Umstand, daß die Isothermen orthogonal zu den Bildern der drei Quadratseiten laufen, kann als Indiz für die Korrektheit der Implementierung gedeutet werden.
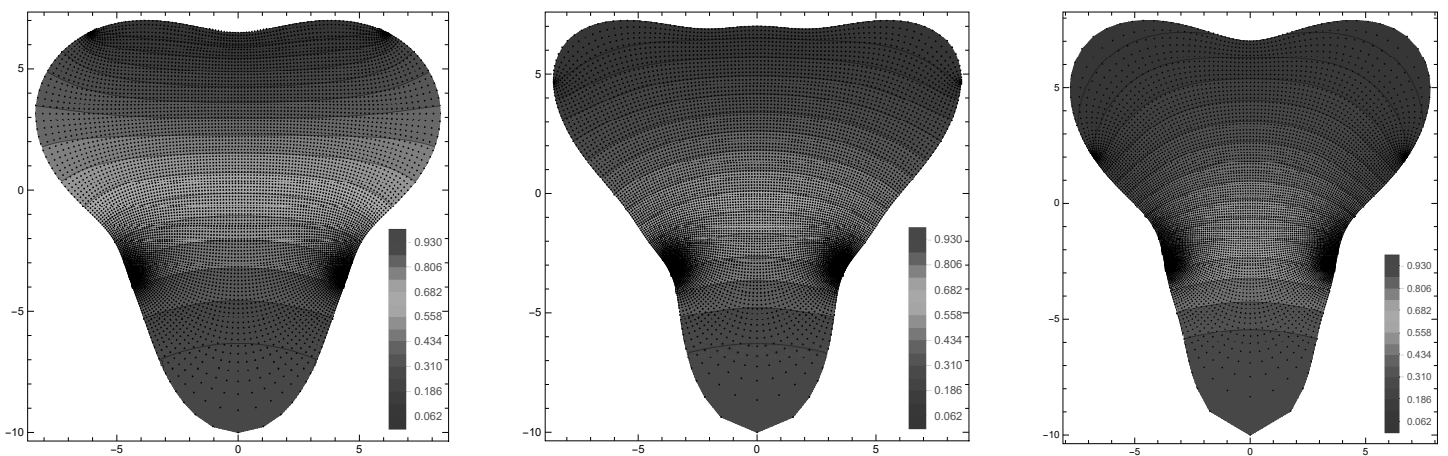

Abbildung 5.2: Temperaturverteilung $(t=50)$ und Isothermen auf den drei Zahngeometrien.

So erfreulich sich die gewonnenen Simulationsergebnisse auch präsentieren (die Erfahrungen am Kreis und dem Quadrat legen zusätzlich nahe, daß sich, abgesehen vom Diskretisierungsfehler, die Fehlergenauigkeit im Bereich des Approximationsfehlers der Ränderzuordnungsfunktion bewegen wird), geben sie doch Anlaß zu einer kritischen Hinterfragung. Da es sich bei Zähnen im allgemeinen um dreidimensionale Gebilde handelt, ist die Qualität der errechneten Temperaturverteilung hinsichtlich einer dreidimensionalen Modellerweiterung zu beurteilen. Der konformen Transformation liegt der Laplace-Operator in kartesischen Koordinaten als Ausgangsüberlegung zu Grunde, interpretiert man diesen in drei kartesischen Raumkoordinaten, so bedeutet eine Reduzierung auf den zweidimensionalen Querschnitt, daß durch Fallenlassen der dritten Ortsableitung gefordert wird, daß sich das Temperaturfeld in dieser Koordinatenrichtung nicht ändert. Die Resultate sind also nur aussagekräftig für den Fall eines unendlich ausgedehnten Rohres, dessen Querschnitt in jedem Punkt der dritten Koordinatenachse auf das zweidimensionale Gebiet $\mathfrak{G}$ führt. Läßt man hingegen $\mathfrak{G}$ um die $\eta$-Achse rotieren, so wird dies auf eine realistischere dreidimensionale Zahngeometrie führen, allerdings wäre dann der Laplace-Operator in Zylinderkoordinaten zu Grunde zu legen. Diesen Überlegungen wird in den folgenden Ausführungen Rechnung getragen.

Es $\operatorname{sei}^{4}$ in kartesischen Koordinaten $(\xi, \eta, \mathfrak{z})$ ein rotationssymmetrisches Gebiet $\mathfrak{G}^{*} \subset \mathbb{R}^{3}$ gegeben, wobei mit der $\eta$-Achse die Rotationsachse bezeichnet werde. Sei weiters mit

$$
f(x+i y)=\xi(x, y)+i \eta(x, y)
$$

eine konforme Abbildung (analytisch oder durch numerische Konstruktion) gegeben, die das Einheitsquadrat der $(x, y)$-Ebene für $\mathfrak{z}=0$ auf den Querschnitt $\mathfrak{G}$ von $\mathfrak{G}^{*}$ in der $\zeta$-Ebene abbildet. Durch Einführung neuer, orthogonaler Koordinaten $(x, y, \varphi)$ mittels

$$
\begin{aligned}
\xi & =\xi(x, y) \sin \varphi \\
\eta & =\eta(x, y) \\
\mathfrak{z} & =\xi(x, y) \cos \varphi
\end{aligned}
$$

\footnotetext{
${ }^{4}$ Da das Symbol $\zeta$ reserviert ist zur Bezeichnung der komplexen $\zeta$-Ebene bzw. die komplexen Zahlen $\zeta=\xi+i \eta$ durchgehend zur Beschreibung der Geometrie in kartesischen Koordinaten verwendet werden, wird hier als dritte Raumkoordinate die kartesische $\mathfrak{z}$-Achse eingeführt.
} 
erhält man, ausgehend von $g_{k l}=\delta_{k l}$ im Falle kartesischer Raumkoordinaten, unter Beachtung des Transformationsgesetzes (4.4) und $\xi_{x}=\eta_{y}, \xi_{y}=-\eta_{x}$ für den kovarianten Fundamentaltensor Abbildung 5.3 die neuen Koordinaten

$$
\bar{g}_{i j}=\left(\begin{array}{ccc}
\xi_{x}^{2}+\eta_{x}^{2} & 0 & 0 \\
0 & \xi_{x}^{2}+\eta_{x}^{2} & 0 \\
0 & 0 & \xi^{2}
\end{array}\right)
$$

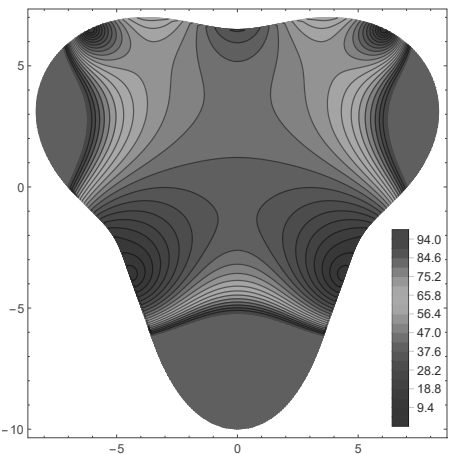

Geometrie 1: $g_{11}=g_{22}$

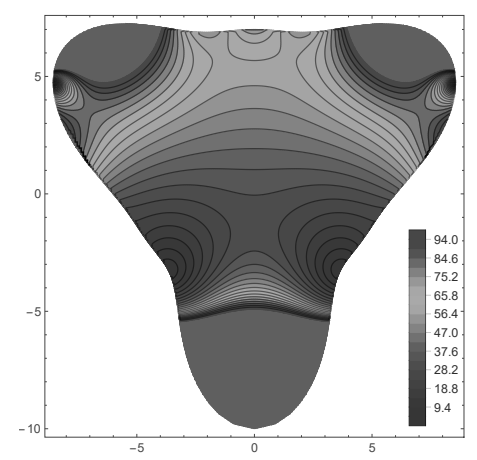

Geometrie 2: $g_{11}=g_{22}$

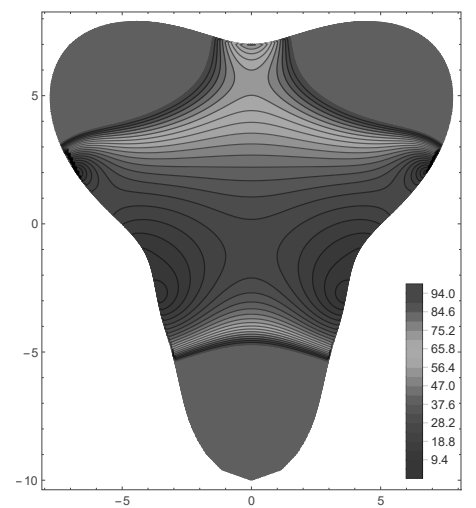

Geometrie 3: $g_{11}=g_{22}$

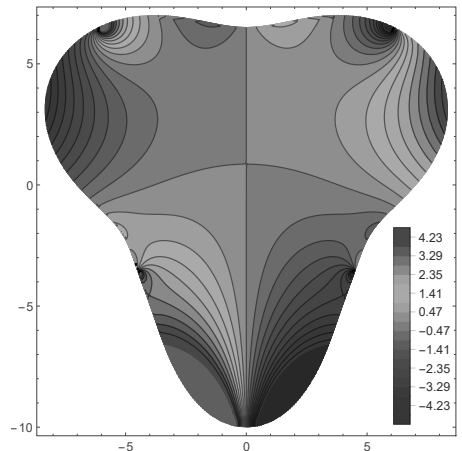

Geometrie 1: $\Gamma_{11}^{1}$

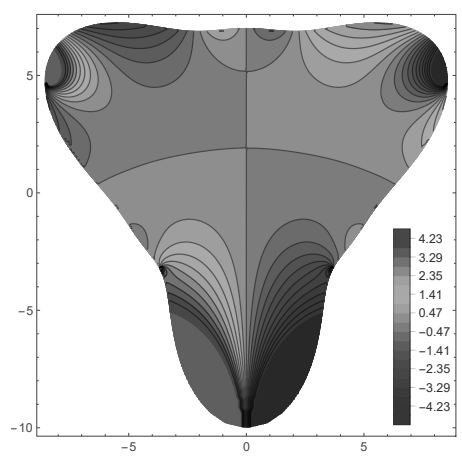

Geometrie 2: $\Gamma_{11}^{1}$

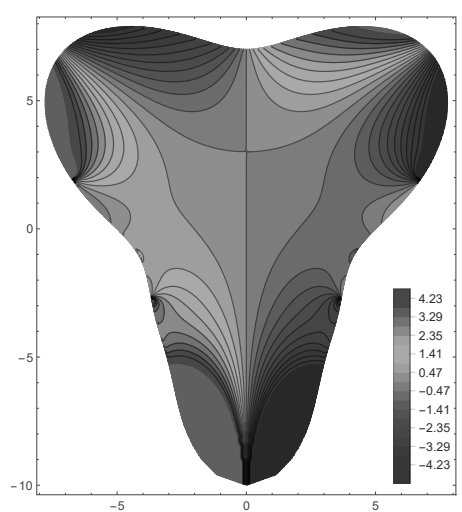

Geometrie 3: $\Gamma_{11}^{1}$

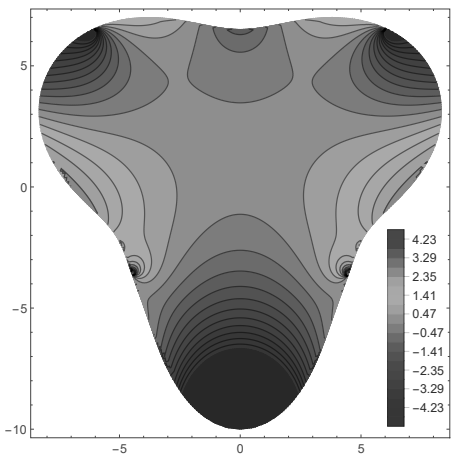

Geometrie 1: $\Gamma_{12}^{1}$

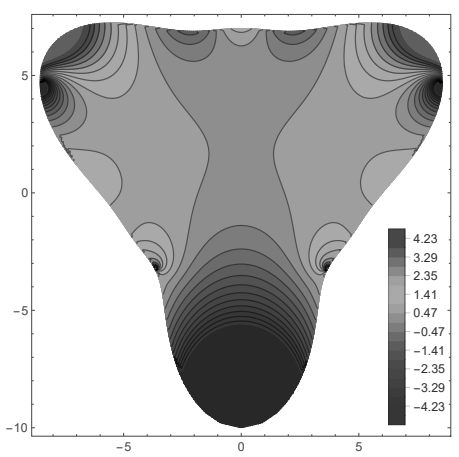

Geometrie 2: $\Gamma_{12}^{1}$

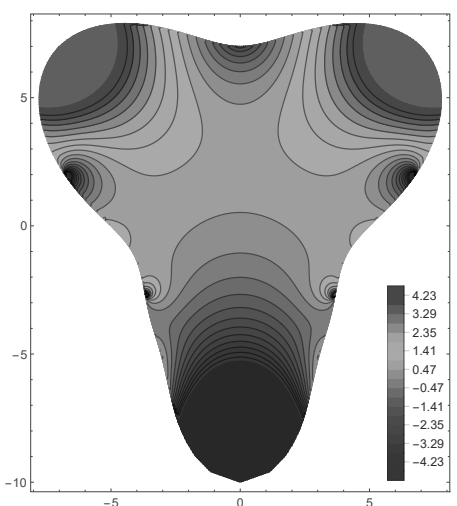

Geometrie 3: $\Gamma_{12}^{1}$

Abbildung 5.3: Der Maßtensor und jeweils zwei der (unabhängigen) Zusammenhangskoeffizienten (4.11) auf den Geometrien $1-3$. 
Man ersieht daraus, daß der Maßtensor vom Drehwinkel $\varphi$ unabhängig ist und es folgt gemäß 4.10 mit der Setzung $\chi=\xi_{x}^{2}+\eta_{x}^{2}$ für den Laplace-Operator in den neuen Koordinaten $(x, y, \varphi)$

$$
\triangle u=\frac{1}{\chi}\left[\frac{\partial^{2} u}{\partial x^{2}}+\frac{\partial^{2} u}{\partial y^{2}}+\frac{1}{\xi}\left(\xi_{x} \frac{\partial u}{\partial x}-\eta_{x} \frac{\partial u}{\partial y}\right)\right]+\frac{1}{\xi^{2}} \frac{\partial^{2} u}{\partial \varphi^{2}},
$$

wobei auf Grund der Forderung nach einem von $\varphi$ unabhängigen Temperaturfeld darin der letzte auftretende Summand fallengelassen werden kann. Man hat also mit (5.2) einen recht einfachen Ausdruck gefunden, was nicht unbedingt zu erwarten war, da 4.10 die Bildung der Ableitungen der Komponenten des kontravarianten Maßtensors sowie jener der Determinante des kovarianten Maßtensors vorschreibt. Wie man sich durch Nachrechnen aber überzeugt, entfallen sämtliche die zweiten Ableitungen $\xi_{x x}$ und $\eta_{x x}$ beinhaltenden Terme ${ }^{5}$. Neben dem Umstand, daß durch die dreidimensionale Erweiterung des Modells ein weitaus realistischeres Szenario geschaffen wird, erweist sich diese Vorgehensweise auch dahingehend als vorteilhaft, als bei der Simulation nur auf der Hälfte des Zahnquerschnitts gerechnet werden muß, was eine Einsparung an Rechenleistung mit sich bringt: Um Diskretisierungspunkte auf der Rotationsachse zu gewährleisten, wählt man für ein Rechengitter $\mathfrak{Q}_{n, n}$ eine ungerade Anzahl $n$ an Stützstellen, Einschränkung auf die rechte Quadrathälfte erbringt eine (nahezu) Halbierung der zu Grunde liegenden Gitterpunkte von $n^{2}$ auf $\left(n^{2}+n\right) / 2$.

Es gilt allerdings zu beachten, daß nunmehr eine andere Geometrie vorliegt und somit (5.2) nur im Inneren eines Gebietes gefordert werden kann, das durch Einschränkung auf die rechte Seite des Einheitsquadrates, $(0,1) \times(-1,1)$, parametrisiert wird. Hinsichtlich der Randbedingungen hat dies zur Folge, daß für Punkte auf der $\eta$-Achse $\nabla_{\mathbf{n}} u=0$ eine mögliche Vorschrift darstellt (andernfalls wären die Isothermen auf der Rotationsachse nicht mehr differenzierbar), die übrigen Randbedingungen können unverändert übernommen werden. Die Plausibilität dieser Forderung ersieht man auch durch Untersuchung des Ausdrucks $\left(\xi_{x} u_{x}-\eta_{x} u_{y}\right) / \xi$ bei Annäherung $\xi \rightarrow 0+$ an die $\eta$-Achse: Um zu einer unbestimmten Form zu gelangen, muß nämlich der Zähler verschwinden, unter Berücksichtigung von $\eta_{x} \rightarrow 0$ (dieser Umstand ist allgemein gültig für bezüglich der $\eta$-Achse symmetrische Gebiete $\left.{ }^{6}\right)$ ist dann $u_{x} \rightarrow 0 \mathrm{zu}$ fordern.
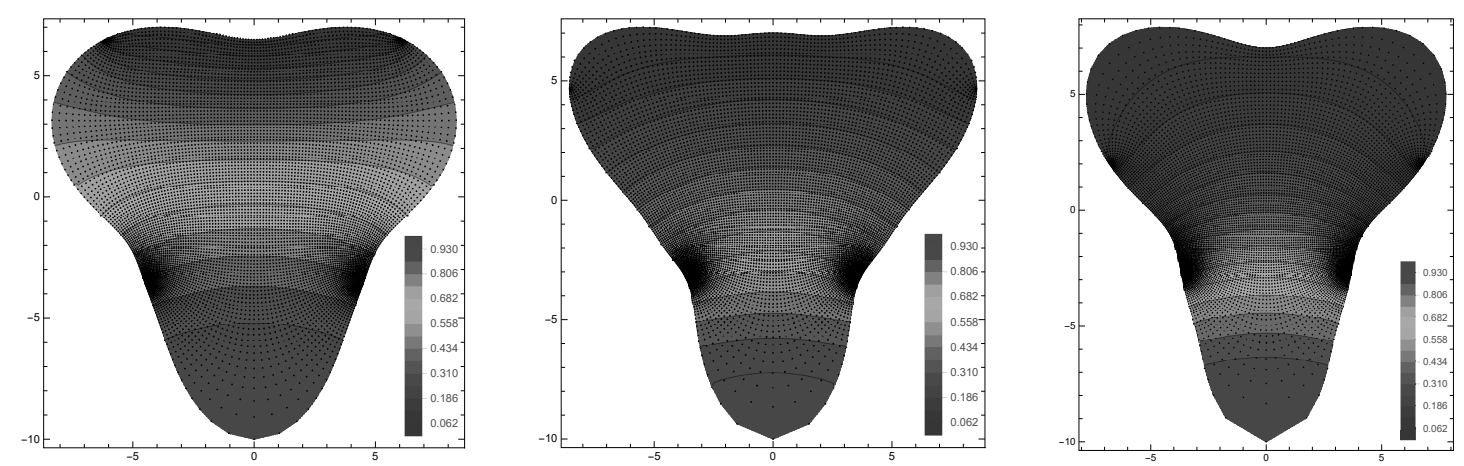

Abbildung 5.4: 3D-Modell mit transformiertem Laplace-Operator: Isothermen für $t=50$.

Simuliert man zunächst testweise die ursprüngliche, zweidimensionale Aufgabenstellung auf der halben Geometrie mit adiabatischer Isolierung der Rotationsachse als neuer Randbedingung, so stimmen die Ergebnisse auf etwa vier Nachkommastellen mit jenen aus Abbildung 5.2 überein.

Eine dreidimensionale Modifikation des Modells — das Temperaturfeld und die Isothermen sind in Abbildung 5.4 dargestellt — bewirkt hingegen qualitativ, daß bei Verwendung des LaplaceOperators (5.2) auf allen drei Geometrien tendenziell mehr Wärme über die Newtonsche Randbedingung auf der Oberseite abgeführt wird. In der Tat bestätigt auch der Vergleich durch Bilden

\footnotetext{
${ }^{5} f^{\prime \prime}$ ist dennoch numerisch als Teil der Gitterinformation für den Eventualfall extern abgespeichert.

${ }^{6}$ Ist $f$ symmetrisch bezüglich der $\eta$-Achse, so gilt $\xi(-x, y)+i \eta(-x, y)=-\xi(x, y)+i \eta(x, y)$, also $\xi(0, y)=0 \forall y$, die $y$-Achse wird auf die $\eta$-Achse abgebildet und dort gilt $\eta_{x}(0, y)=\lim _{\delta \rightarrow 0}[\eta(\delta, y)-\eta(-\delta, y)] / 2 \delta=0$. Daraus kann man auf der Rotationsachse wegen $f^{\prime} \neq 0$ dann auch noch $\xi_{x}(0, y) \neq 0$ folgern.
} 
der arithmetischen Temperaturmittel zum Zeitpunkt $t=50$ in allen Gitterpunkten von $\mathfrak{Q}_{101,101}$ eine teilweise sehr signifikante Reduktion der Durchschnittstemperaturen um 3\% (Geometrie 1; von 0,48 auf 0,46), 30\% (Geometrie 2; von 0, 4 auf 0,28) bzw. 44\% (Geometrie 3; von 0,34 auf 0,19) gegenüber dem Ausgangsmodell. Vor allem bei den letzten beiden Querschnitten enscheidet sich also, ob das Parodont durch eine mangelhafte Modellbildung gegrillt wird oder nicht.

Bei der Implementierung des neuen Modells ist ferner zu beachten, daß nun neben den zweiten Ortsableitungen auch die ersten Ableitungen des Temperaturfeldes auftauchen und entsprechend diskretisiert werden müssen. Um die selbe Fehlerordnung wie bei Diskretisierung der Randbedingungen zu erzielen, ist es zweckmäßig, sich an Beispiel 2 aus Abschnitt 1.5 zu orientieren, für die Ableitungen in den Punkten im Inneren des Rechengitters bedeutet dies exemplarisch für die $x$ Richtung, wenn dabei eine ungerade Anzahl $n$ an äquidistanten Diskretisierungspunkten auf dem gesamten Quadrat mit $n_{R}=\frac{n+1}{2}$ angenommen werde,

$$
\frac{\partial u\left(x_{i}, y_{j}, t\right)}{\partial x} \approx \begin{cases}\frac{-u_{i+2, j}(t)+8 u_{i+1, j}(t)-8 u_{i-1, j}(t)+u_{i-2, j}(t)}{12 \Delta}, & i=n_{R}+2, \ldots n-2 \\ \frac{-3 u_{i-1, j}(t)-10 u_{i, j}(t)+18 u_{i+1, j}(t)-6 u_{i+2, j}(t)+u_{i+3, j}(t)}{12 \Delta}, & i=n_{R}+1 \\ \frac{-u_{i-3, j}(t)+6 u_{i-2, j}(t)-18 u_{i-1, j}(t)+10 u_{i, j}(t)+3 u_{i+1, j}(t)}{12 \Delta}, & i=n-1 .\end{cases}
$$

Wirft man abschließend einen Blick auf die Konvergenz- bzw. Konsistenzverhältnisse des rotationssymmetrischen Modells, so muß beachtet werden, daß ein Vergleich der Durchschnittstemperaturen bei Verdoppelung der Anzahl der Rechengitterpunkte nur bedingt Aussagekraft besitzt. Dies ist in dem Umstand begründet, daß die Bildpunkte auf der Geometrie nicht mehr äquidistant liegen und somit eine ungewollte Gewichtung der Temperaturen auftritt. Ähnliches gilt bei Untersuchung der Minima bzw. Maxima bei Gitterverfeinerung, da die Extrema auch auf den neu hinzukommenden Gitterpunkten zu liegen kommen können, wodurch ein Vergleich unzulässig wird. Da der Ursprung wegen $f(0)=0$ allen Gittern gemeinsam ist, kann die dortige Veränderung der Temperatur als brauchbare Referenz dienen, die Ergebnisse sind in Tabelle 5.1 angeführt.

\begin{tabular}{|c||c|c|c|c|c|}
\hline \hline Geometrie & Anzahl Gitterpunkte & Ursprung & Abweichung & Durchschnitt & Abweichung \\
\hline \hline & $1.326(26 \times 51)$ & 0.507636 & - & 0.463739 & - \\
\hline 1 & $5.151(51 \times 101)$ & 0.507906 & $0.05 \%$ & 0.464922 & $0.25 \%$ \\
\hline \hline & $20.301(101 \times 201)$ & 0.507932 & $0.005 \%$ & 0.465391 & $0.1 \%$ \\
\hline & 1.326 & 0.297975 & - & 0.274446 & - \\
\hline & 5.151 & 0.298824 & $0.285 \%$ & 0.276064 & $0.59 \%$ \\
\hline \hline & 20.301 & 0.298964 & $0.047 \%$ & 0.276494 & $0.16 \%$ \\
\hline 3 & 1.326 & 0.197521 & - & 0.187529 & - \\
\hline & 5.151 & 0.199831 & $1.17 \%$ & 0.190526 & $1.6 \%$ \\
\hline
\end{tabular}

Tabelle 5.1: Zur Konvergenz des Rotationsmodells auf den drei Geometrien $(t=50)$. Die Abweichung errechnet sich durch Quotientenbildung mit dem Wert des Modells mit verdoppeltem Gitterabstand.

Daraus kann abgeleitet werden, daß die Kreisnähe der Geometrie auch global Einfluß auf die Konvergenzgeschwindigkeit nimmt. Eine weiter zunehmende Gitterverfeinerung, also die Halbierung der Gitterpunktabstände unter Verwendung von $201 \times 401$ Rechenpunkten, ist im übrigen auf dem zu allen Berechnungen durchgehend verwendeten Standard-Notebook mit 8 GB Arbeitsspeicher nicht mehr gelungen. In diesem Fall verabschiedet sich die Routine NDSolve, ohne die konkreten Auslöser der ungeplanten Absenz mitzuteilen (definitiv ein Speicherproblem). Als Grund dafür ist neben dem Umstand, daß dann ein gekoppeltes System von etwa 80.000 gewöhnlichen Differentialgleichungen zu lösen ist, vor allem auch im Auftreten der zusätzlichen Terme im Laplace-Operator anzusehen. 


\subsection{Parameterüberlegungen und Modellerweiterungen}

Das vorgestellte Grundmodell ist vermöge der instationären Wärmeleitungsgleichung in der Lage, Vorhersagen über die Wärmeausbreitung auf der Geometrie unter Berücksichtigung der Rotationssymmetrie zu tätigen. Erwägungen hinsichtlich der physikalischen Größen, der Randbedingungen, der eingebrachten Wärmequelle sowie möglicher Modellerweiterungen beschließen dieses Kapitel. Die exakte Identifikation der Parameter bleibt - ebenso wie das Durchführen numerischer Experimente zum Abgleich mit gegebenenfalls vorhandenen Messdaten — dem Anwender überlassen.

\subsubsection{Erhebung realistischer Parameter}

Wie ein Blick auf die instationäre Wärmeleitungsgleichung (1.1) zeigt, ist dort der Thermische Diffusionskoeffizient $\kappa$ alleinverantwortlich für die Geschwindigkeit der Wärmeausbreitung im Inneren der Geometrie. In den bisherigen Betrachtungen wurde der Bequemlichkeit halber von $\kappa=1$ ausgegangen und daß man mit diesem Ansatz per Zufall sogar eine ganz passable Ersteinschätzung getroffen hat, bestätigt sich durch einen Blick in die Fachliteratur [26, Fig. 5]. Dort werden für Dentin und Enamel Werte angegeben, die sich im Bereich $\kappa \approx 2-5 \times 10^{-7}\left[\mathrm{~m}^{2} \mathrm{~s}^{-1}\right]$ bewegen. Legt man, wie sich aus der Skalierung der Geometrien ergibt, $[\mathrm{mm}]$ als Einheit zu Grunde, so ist es gerechtfertigt, mit $\kappa=0,3\left[\mathrm{~mm}^{2} \mathrm{~s}^{-1}\right]$ als Parameter in den Simulationslauf zu gehen, dem man beispielsweise eine konstante Anfangstemperaturverteilung von $u_{0}=36{ }^{\circ} \mathrm{C}$ vorschreiben kann.

Denkbar ist auch, verschiedene Bereiche im Inneren der Geometrie zu definieren, die der Pulpa, dem Dentin und dem Schmelz entsprechen und dort mit verschiedenen Diffusionskoeffizienten zu operieren. Dies ist aber nur zulässig, sofern diese in den Bereichen konstant gehalten werden, da sich bei Ortsabhängigkeit von $\kappa$ die Gestalt der rechten Seite von (1.1) ändern und Ortsableitungen von $\kappa$ beinhalten würde. Ähnliche Überlegungen sind anzustellen, will man den Koeffizienten zusätzlich abhängig machen von der in einem Punkt vorherrschenden Temperatur. In diesen Fällen ist es zielführend, sich die Zusammenhänge zunächst in koordinatenfreier Weise zu verdeutlichen und dann mit Hilfe der Transformationsgesetze in den speziellen, konformen Koordinaten zu realisieren.

Hinsichtlich der Randbedingungen gilt es zu überlegen, daß bei Abwesenheit von Wärmequellen im Inneren und konstant vorgegebener Anfangstemperatur kein Zu- oder Abfluß über den Geometrierand erfolgen sollte. Zudem ist das Vorschreiben fixer Randtemperaturen über DirichletBedingungen jedenfalls im Bereich der Zahnwurzel nicht zielführend, da sich ja dort ein Temperaturprofil in Abhängigkeit einer später im Bereich der Rotationsachse einzubringenden Wärmequelle einstellen soll. Mit diesen Überlegungen kommt

$$
\nabla_{\mathbf{n}} u= \begin{cases}0, & x=0, y \in(-1,1) \\ \alpha_{W}\left(u_{0}-u\right), & x \in(0,1), y=-1 \\ \alpha_{W}\left(u_{0}-u\right), & x=1, y \in(-1,1) \\ \alpha_{K}\left(u_{0}-u\right), & x \in(0,1), y=1,\end{cases}
$$

worin bei Geometrie 3 der Bequemlichkeit halber der Übergang von Zahnwurzel zu Zahnkrone im Punkt $(1,1)$ des Rechengebietes zu liegen kommen soll. Dies stellt aber keinerlei Einschränkung dar, selbstverständlich können durch entsprechende Routinen beliebige Randbedingungen für beliebige Bereiche der Berandung implementiert werden. Die positiven Parameter $\alpha_{W}$ und $\alpha_{K}$ führen dabei Wärme ab, solange sich die Randtemperatur oberhalb einer Referenztemperatur befindet.

Die Wärmeübergangskoeffizienten ( $\alpha_{K}$ von Enamel in Luft im Bereich der Krone bzw. $\alpha_{W}$ vom Cementum in das zahnexterne Knochengewebe im Bereich der Wurzel) müssen experimentell bestimmt werden. Einen Anhaltspunkt über die ungefähre Größenordnung erhält man in [24, S.1640]: Hier wird im Zuge numerischer Experimente ausgehend von $\approx 10^{3}\left[\mathrm{Wm}^{-2} \mathrm{~K}^{-1}\right]$ der Übergangsparameter jeweils um eine Zehnerpotenz verkleinert, was einer Annäherung an adiabatische Isolierung entspricht. Unterstellt man einen geringeren Wärmeübergang im Bereich der Zahnkrone, so scheinen $\alpha_{W}=10^{-4}\left[\mathrm{Wmm}^{-2} \mathrm{~K}^{-1}\right]$ und $\alpha_{K}=10^{-5}\left[\mathrm{Wmm}^{-2} \mathrm{~K}^{-1}\right]$ plausible Ausgangsparameter. 


\subsubsection{Modellierung der Wärmequelle}

Die thermische Behandlung sieht die Applikation einer Wärmequelle mit Hilfe einer Laserapparatur vor, die entlang des Wurzelkanals auf- und abbewegt wird und dabei den umliegenden Bereich erwärmt. Da dem Wurzelkanal die $\eta$-Rotationsachse entspricht kann in Erwägung gezogen werden, diesen Vorgang durch entsprechende Abänderung der dortigen Randbedingung zu modellieren, was erreicht werden könnte durch den Übergang von $\nabla_{\mathbf{n}} u=0 \mathrm{zu} \nabla_{\mathbf{n}} u=\alpha(0, \eta, t)$. Diese Vorgehensweise bringt aber Nachteile mit sich: Einerseits steht man dann wieder vor der Schwierigkeit der Parameteridentifikation einer nunmehr zeit- und ortsabhängigen Randbedingung, zum anderen sind wegen des Diskretisierungsprozesses Probleme numerischer Natur zu erwarten und schließlich kann der Umstand, daß der zu modellierende Laserimpuls eine gewisse, justierbare Streubreite im Sinne einer Tiefenwirkung haben soll, nur in wenig zufriedenstellender Weise nachgebildet werden.

Ein alternativer und auch flexiblerer Ansatz bei der Modellierung des Laserimpulses besteht darin, daß die Wärmezufuhr nicht über den Rand Eingang findet, sondern in Form eines zeit- und ortsabhängigen Quellterms im Inneren der Geometrie berücksichtigt wird,

$$
\sigma(\xi, \eta, t)=u_{a m p} \cdot \exp \left[\frac{-\xi^{2}-\left(\eta-\eta_{0}-v t\right)^{2}}{s}\right]
$$

Bei einem solchen Quellterm handelt es sich um eine Invariante (eine Transformation bei Wechsel der Koordinaten muß also nicht gesondert untersucht werden), die zur rechten Seite von Gleichung (1.1) addiert wird. Ein derartiger Basisimpuls liegt somit in Gestalt einer entlang der $\eta$-Achse mit Geschwindigkeit $v$ nach unten $(v<0)$ oder oben $(v>0)$ wandernden Glockenkurve mit Amplitude $u_{a m p}$, Aufpunkt $\eta_{0}$ und Streubreite $s$ vor, durch eine entsprechende Implementierung mittels der Routine Which kann das Signal in Ort und Zeit nach Belieben variiert werden (etwa Auf- und Abwandern eines pulsierenden Signals). Die Quelle ist numerisch gutartig und läßt zudem eine Justierung der Eindringtiefe über den Parameter $s$ zu, orientiert man sich an der zweidimensionalen Normalverteilung, so ließe sich durch zusätzliche Einführung eines Korrelationskoeffizienten prinzipiell auch noch eine bevorzugte Richtung der Quelle auszeichnen. Auf der Rotationsachse selbst wird sich die Temperatur weiterhin durch Diskretisieren der Randbedingung $\nabla_{\mathbf{n}} u=0$ einstellen.

\subsubsection{Ergebnisse}

Wie schon bei den Wärmeübergangskoeffizienten bleibt auch die korrekte Identifikation der Laserparameter dem Anwender durch Abstimmen experimentell gewonnener Daten mit dem Simulationsexperiment überlassen. Es ist aber das Modell durch die oben erfolgten Betrachtungen und Erweiterungen bereits zu einem solchen Grad entwickelt worden, daß abschließend eine Simulation präsentiert werden soll, die deren Leistungsfähigkeit aufzeigen kann.

Eine konstant abgebende Wärmequelle mit $u_{a m p}=400$ und $s=2 / 10$ wandere dazu in der rotationssymmetrischen Geometrie 3 mit $v=-1 / 5$ für eine Zeitdauer von $60 s$ von $\eta_{0}=5$ aus entlang der $\eta$-Achse abwärts in Richtung des Punktes $(0,-7)$. Der Simulation mit $101 \times 201$ Gitterpunkten liegen als Anfangstemperatur $u_{0}=36$ und als weitere Parameter $\kappa=3 / 10, \alpha_{W}=10^{-4}$ und $\alpha_{L}=10^{-5}$ zu Grunde, Abbildung 5.5 zeigt unterhalb der resultierenden Temperaturprofile auch die Temperaturverhältnisse auf der Berandung in Abhängigkeit des Winkels $\theta \in[-\pi / 2, \pi / 2]$. Dort ist die Kenntnis der Temperaturverteilung deshalb von besonderem Interesse, weil sie dem behandelnden Mediziner Einsichten über eine eventuell eintretende Schädigung des Parodonts ermöglicht. 

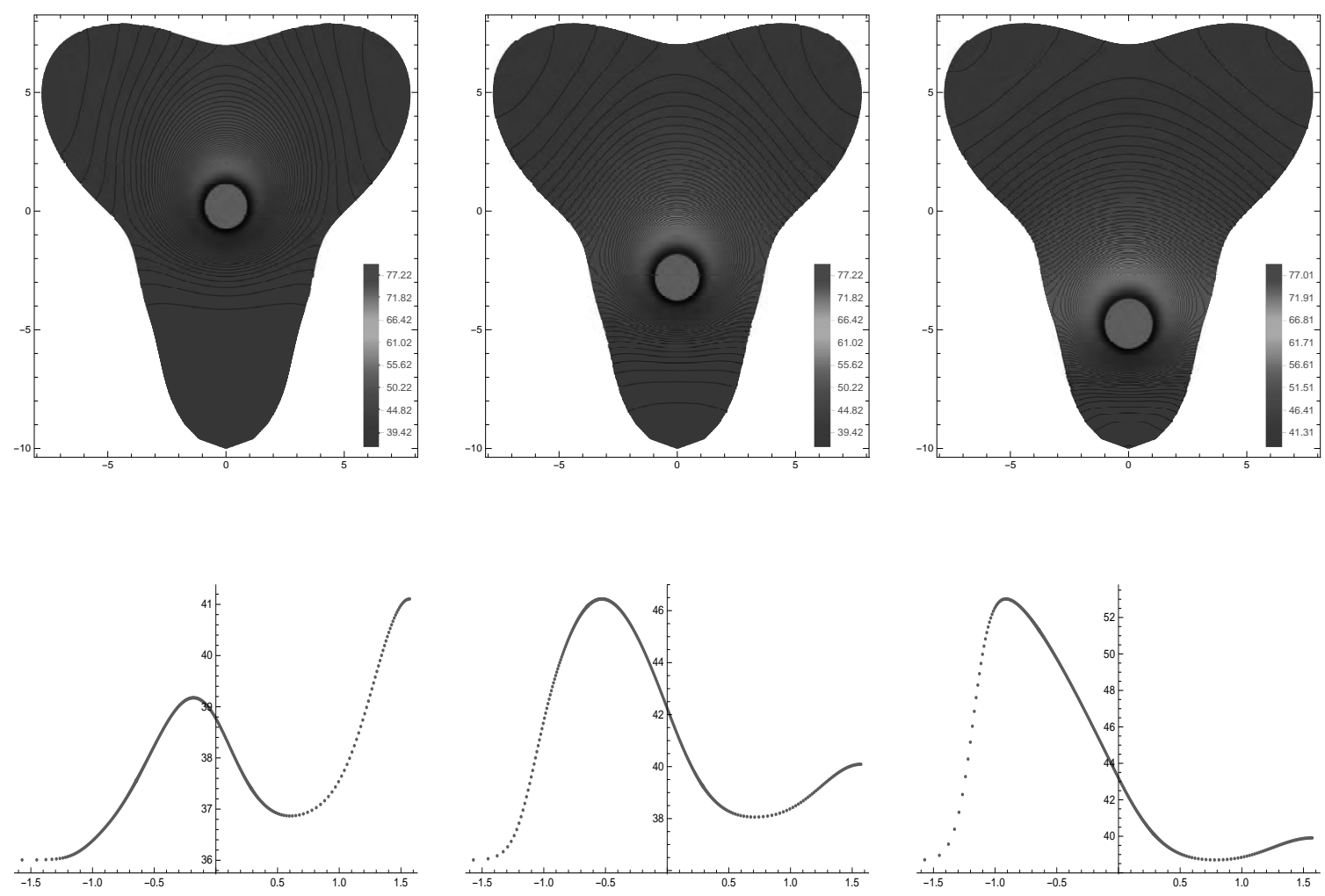

$t=25$

$t=40$

$t=50$

Abbildung 5.5: Vom Rotationsmodell auf Geometrie 3 erzeugte Isothermen einer vom Punkt $(0,5)$ in den Punkt $(0,-7)$ abwärts bewegten Wärmequelle (oben) sowie zugehörige Temperaturverteilungen auf der Berandung als Entscheidungsgrundlage zur Justierung der Laserquelle (unten).

\subsubsection{Inhomogenitäten und Anisotropien}

Zum Abschluß der Betrachtungen am Zahnmodell soll, ohne daß die folgenden Aspekte Eingang in die Implementierung finden, die Auszeichnung einer bevorzugten Richtung der Wärmeleitung sowie die Berücksichtigung unterschiedlicher Materialeigenschaften diskutiert werden.

Dazu seien wieder $(\xi, \eta, \mathfrak{z})$ kartesische Koordinaten eines bezüglich der $\eta$-Achse rotationssymmetrischen Gebietes $\mathfrak{G}^{*} \subset \mathbb{R}^{3}$. Gesucht in $\mathfrak{G}^{*}$ sei (mit den üblichen Randbedingungen) die Lösung einer modifizierten Wärmeleitungsgleichung, gegeben in koordinatenfreier Formulierung durch

$$
\frac{\partial u}{\partial t}=\kappa \operatorname{div}(\bigvee \mathfrak{D} \otimes \operatorname{grad} u)+\sigma .
$$

Mit $\mathfrak{D}$, dem Diffusionstensor, taucht eine neue Größe in der Wärmeleitungsgleichung auf. Ausgehend von seiner 2-fach kontravarianten, symmetrischen Natur erfolgt eine Überschiebung mit dem (kovarianten) Temperaturgradienten, auf das Resultat, ein kontravariantes Vektorfeld, wirkt anschließend die Divergenz. Wird $\mathfrak{D}$ in kartesischen Koordinaten mit (nicht notwendigerweise konstanten) Koeffizienten $d^{i j}$ angesetzt, dann sind bei konstantem $\kappa$ die Materialeigenschaften homogen, die Wärme breitet sich jedoch anisotrop aus (die ausgezeichneten Richtungen können dabei zusätzlich von Punkt zu Punkt variieren). Hängt der thermische Diffusionskoeffizient ebenfalls vom Ort ab (die nicht-konstante aber skalare Funktion $\kappa(\xi, \eta, \mathfrak{z})$ steht dann im Inneren der Divergenz), kann damit die inhomogene Wärmeausbreitung beschrieben werden. Man rechnet nach, daß auf Grund 
der Produktregel dann zusätzlich zum in konkreten Koordinaten realisierten Laplace-Operator ein additiver Term der Form $g^{i j} \frac{\partial \kappa}{\partial x_{j}} \frac{\partial u}{\partial x_{i}}$ auftritt. Gleichung $\sqrt{1.1}$ ist also der Spezialfall für $\kappa(\xi, \eta, \mathfrak{z}) \equiv \kappa$ und dem Fundamentaltensor als Diffusionstensor. Wir betrachten 2 weitere Spezialfälle.

1. Homogene Anisotropie normal zur Rotationsachse. In diesem Fall kann $\mathfrak{D}$ auf dem rotationssymmetrischen Gebiet $\mathfrak{G}^{*}$ in kartesischen Koordinaten mit folgender Begründung in der Form

$$
d_{i j}=\left(\begin{array}{ccc}
\delta & 0 & 0 \\
0 & 1 & 0 \\
0 & 0 & \delta
\end{array}\right)
$$

angesetzt werden: Wenn eine verstärkte Wärmeausbreitung normal zur Rotations-Achse auf Grund der Zahnbeschaffenheit (das Vorhandensein feiner Kanäle im Dentin) angenommen werden kann, ist $\delta>1$ für die $\xi$ - und $\mathfrak{z}$-Richtung der für die Anisotropie zuständige Steuerparameter. In Richtung der Rotationsachse erfolgt die Wärmeleitung demnach mit dem Wert $\kappa$, axial resultiert ein $\delta$ faches von $\kappa$ als Diffusionskoeffizient. Wir haben außerdem den Diffusionstensor der Bequemlichkeit halber in kovarianten Koordinaten angegeben, wohl wissend, daß im kartesischen Ausgangsfall kein Unterschied zwischen $d_{i j}$ und $d^{i j}$ besteht. Mit Transformation 5.1 wird dann gemäß 4.3)

$$
\bar{d}_{i j}=\left(\begin{array}{ccc}
\delta \xi_{x}^{2}+\eta_{x}^{2} & \xi_{x} \eta_{x}(1-\delta) & 0 \\
\xi_{x} \eta_{x}(1-\delta) & \xi_{x}^{2}+\delta \eta_{x}^{2} & 0 \\
0 & 0 & \delta \xi^{2}
\end{array}\right)
$$

Mit diesem Ausdruck sind die kovarianten Koordinaten des Diffusionstensors gefunden, für die noch durchzuführende Verjüngung zieht man hoch zu kontravarianten Indizes ${ }^{7}$ und erhält

$$
\bar{d}^{i j}=\frac{1}{\chi^{2}}\left(\begin{array}{ccc}
\delta \xi_{x}^{2}+\eta_{x}^{2} & \xi_{x} \eta_{x}(1-\delta) & 0 \\
\xi_{x} \eta_{x}(1-\delta) & \xi_{x}^{2}+\delta \eta_{x}^{2} & 0 \\
0 & 0 & \frac{\delta \chi^{2}}{\xi^{2}}
\end{array}\right) .
$$

In den neuen Koordinaten $(x, y, \varphi)$ kann dann mit $\bar{g}=\xi^{2} \chi^{2}$ und

$$
\kappa \operatorname{div}(\bigvee \mathfrak{D} \otimes \operatorname{grad} u)=\frac{\kappa}{\sqrt{|\bar{g}|}} \frac{\partial}{\partial \bar{x}_{i}}\left(\sqrt{|\bar{g}|} \bar{d}^{i j} \frac{\partial u}{\partial \bar{x}_{j}}\right)
$$

eine verallgemeinerte Wärmeleitungsgleichung in der Form

$$
\begin{aligned}
& \frac{\partial u}{\partial t}=\frac{\kappa}{\xi \chi}\left\{\frac{\partial}{\partial x}\right. {\left[\xi\left(\frac{\delta \xi_{x}^{2}+\eta_{x}^{2}}{\chi} \frac{\partial u}{\partial x}+\frac{\xi_{x} \eta_{x}(1-\delta)}{\chi} \frac{\partial u}{\partial y}\right)\right]+} \\
& \frac{\partial}{\partial y} {\left[\xi\left(\frac{\xi_{x} \eta_{x}(1-\delta)}{\chi} \frac{\partial u}{\partial x}+\frac{\xi_{x}^{2}+\delta \eta_{x}^{2}}{\chi} \frac{\partial u}{\partial y}\right)\right]+} \\
&\left.\frac{\partial}{\partial \varphi}\left[\frac{\delta \chi}{\xi} \frac{\partial u}{\partial \varphi}\right]\right\}+\sigma
\end{aligned}
$$

formuliert werden. Wie man durch Nachrechnen bestätigt, führt die Setzung $\delta=1$ auf den isotropen Fall von Gleichung (5.2) zurück. Fordert man nun wieder ein von $\varphi$ unabhängiges Temperaturfeld, differenziert aus und sortiert die Terme, so erhält man eine zweidimensionale Gleichung

$$
\begin{aligned}
\frac{\partial u}{\partial t}=\frac{\kappa}{\xi \chi}\left\{\frac{\xi\left(\delta \xi_{x}^{2}+\eta_{x}^{2}\right)}{\chi} \frac{\partial^{2} u}{\partial x^{2}}+\frac{\xi\left(\xi_{x}^{2}+\delta \eta_{x}^{2}\right)}{\chi} \frac{\partial^{2} u}{\partial y^{2}}+\frac{2 \xi \xi_{x} \eta_{x}(1-\delta)}{\chi} \frac{\partial^{2} u}{\partial x \partial y}+\right. \\
\frac{\partial u}{\partial x} \cdot\left[\frac{\partial}{\partial x}\left(\frac{\xi\left(\delta \xi_{x}^{2}+\eta_{x}^{2}\right)}{\chi}\right)+\frac{\partial}{\partial y}\left(\frac{\xi \xi_{x} \eta_{x}(1-\delta)}{\chi}\right)\right]+ \\
\left.\frac{\partial u}{\partial y} \cdot\left[\frac{\partial}{\partial x}\left(\frac{\xi \xi_{x} \eta_{x}(1-\delta)}{\chi}\right)+\frac{\partial}{\partial y}\left(\frac{\xi\left(\xi_{x}^{2}+\delta \eta_{x}^{2}\right)}{\chi}\right)\right]\right\}+\sigma .
\end{aligned}
$$

$7 \bar{d}^{i j}=g^{i h} g^{j k} \bar{d}_{h k}$ 
Für $\delta=1$ reduziert sich die Gleichung wieder auf (5.2). Bei genauerer Betrachtung ersieht man einerseits, daß sich der Implementierungsaufwand zwar erhöht, prinzipiell aber keine neu zu berechnenden metrischen Größen auftreten. Andererseits muß dem Auftreten von gemischten partiellen Ableitungen durch Verwendung der Moleküle aus Beispiel 4 im Abschnitt 1.5 begegnet werden.

2. Inhomogene Isotropie/Anisotropie mit Kugelsymmetrie. Wird beachtet, daß der Bereich der Pulpa eine geringere Wärmeleitfähigkeit als Dentin besitzt und der Verlauf der das Dentin durchsetzenden Kanäle als radial nach außen verlaufend angenommen, kann $\mathfrak{D}$ in Kugelkoordinaten durch

$$
\mathfrak{D}=\kappa(r, \varphi, \vartheta)\left(\begin{array}{ccc}
\delta & 0 & 0 \\
0 & r^{2} \sin ^{2} \vartheta & 0 \\
0 & 0 & r^{2}
\end{array}\right)
$$

angesetzt werden. Dabei ist $\kappa(r, \varphi, \vartheta)$ das Skalarfeld des vom Ort abhängenden thermischen Diffusionskoeffizienten und die radiäre Anisotropie zur Berücksichtigung der Dentintubuli kommt durch Abänderung des ersten Elementes des kovarianten Maßtensors in $\delta>1$ zum Ausdruck. Im Bereich der Pulpa ist $\delta=1 \mathrm{zu}$ setzen, hier soll die Wärmeleitung isotrop sein. Wir transformieren zunächst $\mathfrak{D}$ von Kugelkoordinaten auf kartesische Koordinaten. Unter Beachtung der Beziehungen

$$
\begin{aligned}
& r=\sqrt{\xi^{2}+\eta^{2}+\mathfrak{z}^{2}} \quad \varphi=\arctan \frac{\eta}{\xi} \quad \vartheta=\arccos \frac{\mathfrak{z}}{\sqrt{\xi^{2}+\eta^{2}+\mathfrak{z}^{2}}} \\
& r_{\xi}=\frac{\xi}{\sqrt{\xi^{2}+\eta^{2}+\mathfrak{z}^{2}}} \quad \varphi_{\xi}=-\frac{\eta}{\xi^{2}+\eta^{2}} \quad \vartheta_{\xi}=\frac{\xi_{\mathfrak{z}}}{\sqrt{\xi^{2}+\eta^{2}}\left(\xi^{2}+\eta^{2}+\mathfrak{z}^{2}\right)} \\
& r_{\eta}=\frac{\sqrt{\xi^{2}+\eta^{2}+\mathfrak{z}^{2}}}{\sqrt{\xi^{2}+\eta^{2}+\mathfrak{z}^{2}}} \quad \varphi_{\eta}=\frac{\xi}{\xi^{2}+\eta^{2}} \quad \vartheta_{\eta}=\frac{\sqrt{\xi^{2}+\eta^{2}}\left(\xi^{2}+\eta^{2}+\mathfrak{z}^{2}\right)}{\sqrt{\xi^{2}+\eta^{2}}\left(\xi^{2}+\eta^{2}+\mathfrak{z}^{2}\right)} \\
& r_{\mathfrak{z}}=\frac{\mathfrak{z}}{\sqrt{\xi^{2}+\eta^{2}+\mathfrak{z}^{2}}} \quad \varphi_{\mathfrak{z}}=\quad 0 \quad \vartheta_{\mathfrak{z}}=-\frac{\sqrt{\xi^{2}+\eta^{2}}}{\xi^{2}+\eta^{2}+\mathfrak{z}^{2}}
\end{aligned}
$$

errechnet sich der Diffusionstensor in kartesischen Koordinaten gemäß $\bar{d}_{i j}=\frac{\partial x_{h}}{\partial \bar{x}_{i}} \frac{\partial x_{k}}{\partial \bar{x}_{j}} d_{h k} \mathrm{zu}$

$$
\mathfrak{D}=\frac{\kappa(\xi, \eta, \mathfrak{z})}{\xi^{2}+\eta^{2}+\mathfrak{z}^{2}}\left(\begin{array}{ccc}
\delta \xi^{2}+\eta^{2}+\mathfrak{z}^{2} & (\delta-1) \xi \eta & (\delta-1) \xi \mathfrak{z} \\
(\delta-1) \xi \eta & \xi^{2}+\delta \eta^{2}+\mathfrak{z}^{2} & (\delta-1) \eta \mathfrak{z} \\
(\delta-1) \xi \mathfrak{z} & (\delta-1) \eta \mathfrak{z} & \xi^{2}+\eta^{2}+\delta \mathfrak{z}^{2}
\end{array}\right)
$$

und mit Transformation (5.1) folgt weiter

$$
\mathfrak{D}=\frac{\kappa(x, y, \varphi)}{\xi^{2}+\eta^{2}}\left(\begin{array}{ccc}
\left(\xi_{x} \eta-\xi \eta_{x}\right)^{2}+\delta\left(\xi_{x} \xi+\eta_{x} \eta\right)^{2} & (\delta-1)\left(\xi_{x} \eta-\xi \eta_{x}\right)\left(\xi_{x} \xi+\eta_{x} \eta\right) & 0 \\
(\delta-1)\left(\xi_{x} \eta-\xi \eta_{x}\right)\left(\xi_{x} \xi+\eta_{x} \eta\right) & \delta\left(\xi_{x} \eta-\xi \eta_{x}\right)^{2}+\left(\xi_{x} \xi+\eta_{x} \eta\right)^{2} & 0 \\
0 & 0 & \xi^{2}\left(\xi^{2}+\eta^{2}\right)
\end{array}\right)
$$

Der Übergang zu kontravarianten Koordinaten erbringt schließlich die 5 nicht-verschwindenden Komponenten des symmetrischen Diffusionstensors, es ist in Koordinaten $(x, y, \varphi)$

$$
\begin{array}{ll}
d^{11}=\frac{\kappa\left[\left(\xi_{x} \eta-\xi \eta_{x}\right)^{2}+\delta\left(\xi_{x} \xi+\eta_{x} \eta\right)^{2}\right]}{\chi^{2}\left(\xi^{2}+\eta^{2}\right)} & d^{22}=\frac{\kappa\left[\delta\left(\xi_{x} \eta-\xi \eta_{x}\right)^{2}+\left(\xi_{x} \xi+\eta_{x} \eta\right)^{2}\right]}{\chi^{2}\left(\xi^{2}+\eta^{2}\right)} \\
d^{12}=d^{21}=\frac{\kappa\left[(\delta-1)\left(\xi_{x} \eta-\xi \eta_{x}\right)\left(\xi_{x} \xi+\eta_{x} \eta\right)\right]}{\chi^{2}\left(\xi^{2}+\eta^{2}\right)} & d^{33}=\frac{\kappa}{\xi^{2}} .
\end{array}
$$

Der nicht-konstante thermische Diffusionskoeffizient $\kappa(x, y, \varphi)$ unterliegt nunmehr als Teil des Diffusionstensors der Divergenzbildung. Man gelangt unter Berücksichtigung der wiederum anzunehmenden Rotationssymmetrie durch Weglassen des Terms $\frac{\partial}{\partial \varphi}\left(\chi \xi d^{33} \frac{\partial u}{\partial \varphi}\right)$ zu einer verallgemeinerten, zweidimensionalen Wärmeleitungsgleichung der Form

$$
\frac{\partial u}{\partial t}=\frac{1}{\xi \chi}\left[\frac{\partial}{\partial x}\left(\xi \chi d^{11} \frac{\partial u}{\partial x}+\xi \chi d^{12} \frac{\partial u}{\partial y}\right)+\frac{\partial}{\partial y}\left(\xi \chi d^{12} \frac{\partial u}{\partial x}+\xi \chi d^{22} \frac{\partial u}{\partial y}\right)\right]+\sigma .
$$


Unter Anwendung der Produktregel kann dies noch als

$$
\begin{aligned}
\frac{\partial u}{\partial t}= & d^{11} \frac{\partial^{2} u}{\partial x^{2}}+2 d^{12} \frac{\partial^{2} u}{\partial x \partial y}+d^{22} \frac{\partial^{2} u}{\partial y^{2}}+ \\
& \frac{1}{\xi \chi}\left[\frac{\partial u}{\partial x}\left(\frac{\partial\left(\xi \chi d^{11}\right)}{\partial x}+\frac{\partial\left(\xi \chi d^{12}\right)}{\partial y}\right)+\frac{\partial u}{\partial y}\left(\frac{\partial\left(\xi \chi d^{12}\right)}{\partial x}+\frac{\partial\left(\xi \chi d^{22}\right)}{\partial y}\right)\right]+\sigma
\end{aligned}
$$

geschrieben werden, was für konstantes $\kappa$ und $\delta=1$ wieder auf Gleichung (5.2) zurückführt. Abermals treten keine neu zu berechnenden metrischen Größen auf, allerdings führen die nunmehr auftretenden Ableitungen der Koordinaten des Diffusionstensors zu einem sehr hohen Grad an Komplexität, die Implementierung wird sich daher entsprechend mühevoll und fehleranfällig gestalten.

Wir schließen mit einem Visualisierungsvorschlag, durch eine Darstellung des Temperaturverlaufes am Rand bei zeitlichem Fortschreiten gewinnt der behandelnde Mediziner intuitive Einsichten über die im Zuge der Laser-Applikation gegebenenfalls entstehenden Gefahrenzonen.

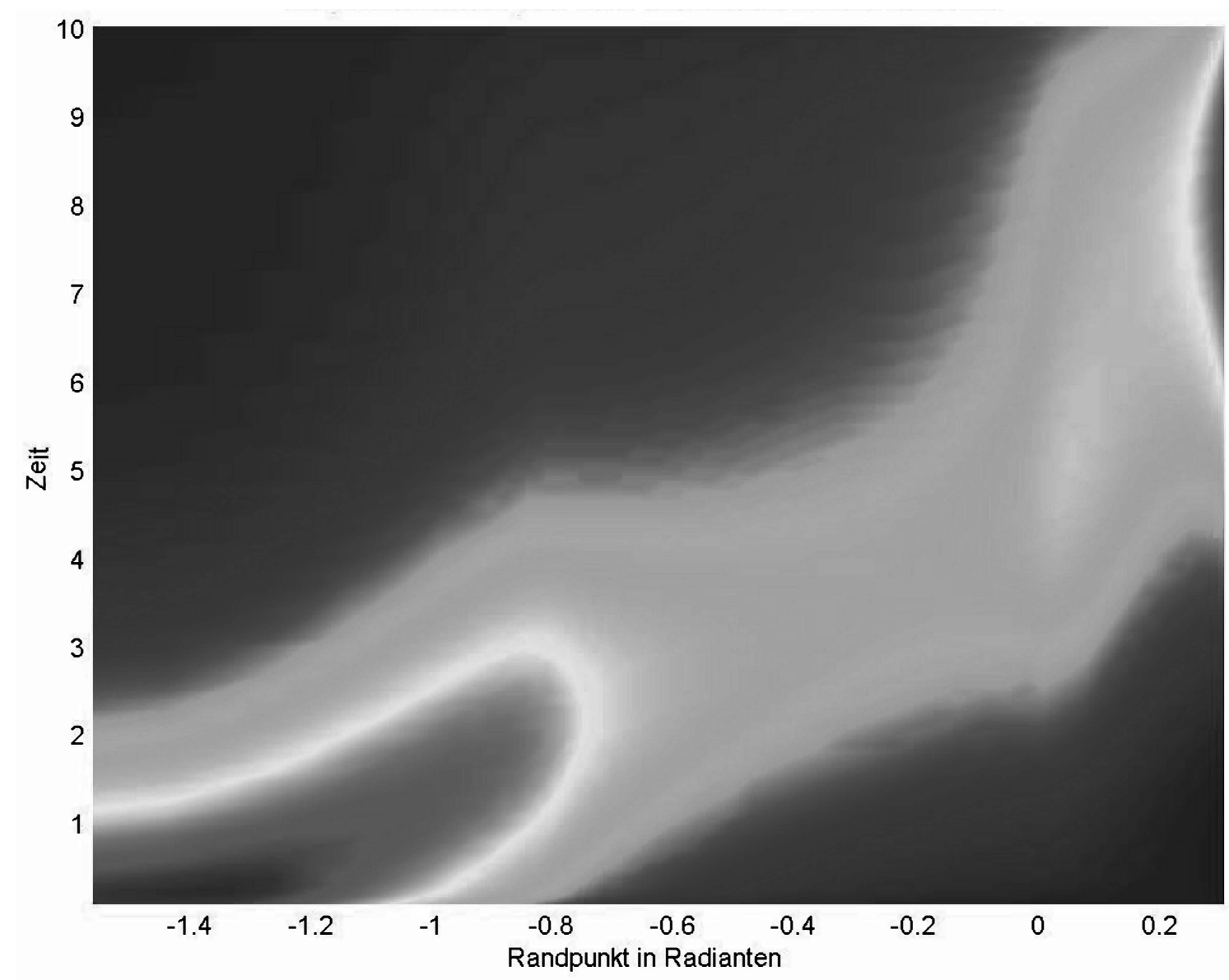

Abbildung 5.6: Temperaturverteilung am Rand im zeitlichen Verlauf. 


\section{Kapitel 6}

\section{Epilog}

Now it's your turn to cry awhile... [Bob Dylan, 2001]

Die dargelegte Vorgehensweise zur Simulation zweidimensionaler Anfangsrandwertprobleme verwendet die konforme Abbildung zur Transformation des Einheitsquadrates über den Einheitskreis auf eine in Betracht stehende Geometrie. Dadurch wird das Innere der Geometrie durch das Quadrat parametrisiert und die Stärken der Linienmethode können nutzbringend eingesetzt werden. Diese Stärken bestehen in der akkuraten Implementierung von Randbedingungen und einer steuerbaren Konvergenzordnung, für den Fall der Wärmeleitungsgleichung konnte an Hand von Referenzlösungen auch dargelegt werden, daß sich - solange die metrischen Größen hinlänglich genau ermittelt sind - durch die Transformation keine weiteren nachteiligen Effekte einstellen. Der Ansatz ist für ebene Geometrien geeignet, kann aber bei Vorliegen von Rotationssymmetrie problemlos auf dreidimensionale Modelle ausgedehnt werden. Die Güte des Ergebnisses läßt sich dabei zurückführen auf die Approximationsgüte der Ränderzuordnungsfunktion, die von Mathematica mit nahezu beliebiger Genauigkeit iteriert werden kann. Die speziell vorgestellte Lösung der Theodorsenschen Integralgleichung stellt dabei nur einen möglichen Zugang zur Konstruktion der Abbildung dar, der in Abhängigkeit von Sternförmigkeit und Kreisnähe des Gebietes konvergiert. Hinsichtlich der Modellgleichungen ist der Ansatz keinerlei Einschränkungen unterworfen, ausgehend von der koordinatenfreien Formulierung ist man in der Lage, unter Berücksichtigung der Transformationsgesetze der Feldgrößen einen Ortsdiskretisierungsprozeß durchzuführen, die Metrik im Diskretisierungspunkt fließt dadurch zusätzlich ein und trägt damit dem speziell gewählten Koordinatensystem Rechnung.

\subsection{Vergleich mit alternativen Finite-Elemente-Verfahren}

Insbesondere die Methode der Finiten Elemente stellt ein etabliertes und sehr aktives Forschungsgebiet dar, mit dem in Konkurrenz zu treten nicht die Absicht dieser Arbeit sein kann. Die im Zuge der Untersuchungen präsentierten Rechengebiete und Ergebnisse können aber in diesem Sinne dennoch zum Vergleich mit den erzielten Resultaten anderer Methoden herangezogen werden.

Aus der Vielzahl der den FE-Ansatz verwendenden Programme wurde zum initialen Vergleich am Quadrat und Kreis speziell die PDE-Toolbox von Matlab herausgegriffen, um zu Einsichten über die prinzipielle Sinnhaftigkeit der Anwendbarkeit des CTDS-Ansatzes zu gelangen. Wenn auch mit jeder neuen Release von Mathworks eine stete Weiterentwicklung der PDE-Toolbox stattfindet, wurde der zu Tage getretenen, quadratischen Fehlerordnung bis zum aktuellen Stand der Dinge hinsichtlich deren Erhöhung offensichtlich noch nicht hinreichend begegnet. Hinterfragt man also dieses vergleichsweise mäßige Abschneiden durch Recherche, so stellt man fest, daß durchaus schon Forschungen und Entwicklungen in diese Richtung stattgefunden haben und stattfinden. Diese haben allerdings bisher noch nicht gänzlich Eingang in die aktuelle Implementierung der Toolbox gefunden, was für zukünftige Versionen aber durchaus erwartet werden kann. Tiefer in die Theorie der 
Finiten Elemente einzudringen würde an dieser Stelle natürlich jeden Rahmen sprengen ${ }^{1}$, im Falle der PDE-Toolbox operiert man jedoch offensichtlich auf den Knotenpunkten der Triangulierung zur Approximation des Temperaturfeldes mit einem Ansatz der Form

$$
u(\xi, \eta)=\sum_{i=1}^{n} c_{i} \Phi_{i}(\xi, \eta) .
$$

Hierbei gilt es unter Berücksichtigung der Randbedingungen die Koeffizienten der Linearkombination geeignet zu bestimmen, die Basisfunktionen $\Phi_{i}$ werden dabei so angesetzt, daß sie nur lokal um einen Knotenpunkt des jeweiligen Elementes nicht verschwindende Werte annehmen. Diesen Ansatz setzt man in die schwache Formulierung des Problems ein und trachtet danach, den resultierenden Fehler zu minimieren. Je nach Natur der zu Grunde liegenden Gleichung führt dies auf ein lineares oder nichtlineares Gleichungssystem für die Koeffizienten $c_{i}$. Bei zusätzlicher Zeitabhängigkeit resultiert daraus, wie im Falle der CTDS-Methode, ein System gewöhnlicher Differentialgleichungen.

Bei vorliegender Unterteilung des Rechengebietes in Dreiecke durch die PDE-Toolbox kann man zur Verbesserung der Konvergenzordnung hinsichtlich der Wahl der Basisfunktionen $\Phi_{i}$ übergehen zu verallgemeinernden Ansätzen vom LAGRANGE-Typ, diese Vorgehensweise benötigt jedoch zusätzliche Übergangsknoten auf den Kanten der Elemente, was umgehend zu einer Erhöhung der Rechendauer und des Bedarfs an Arbeitsspeicher führt. Der Zugang ist vergleichbar mit jenem aus Abschnitt 1.5, also der Verwendung einer höheren Anzahl an Nachbarpunkten zur Approximation der Ortsableitung. Neben den Lagrange-Polynomen kommen bei der Methode der Finiten Elemente bei Vorliegen einer Triangulierung auch höhergradige BERNsTEIN-Polynome zum Einsatz.

Zusätzliche Möglichkeiten zur Steigerung der Genauigkeit ergeben sich, wenn an Stelle der Triangulierung zur Unterteilung der betrachteten Geometrie Viereck-Elemente Verwendung finden. Man erhält bilineare, biquadratische, noch höhergradige oder entsprechend gemischte Ansatzfunktionen mit besserer Approximationsgüte als sie bei gleichem Polynomgrad mit Dreieck-Elementen erzielbar ist. Wegen der geringeren Speicheranforderung erwähnenswert sind auch noch die Elemente der SERENDIPITY-Klasse. Für viereckige Elemente lassen sich außerdem die für den eindimensionalen Fall entwickelten Ansatzfunktionen durch Produktbildung verallgemeinern, sodaß neben den verschiedensten Polynomansätzen auch Splines oder NURBS Verwendung finden können. Ein Mischen der Element-Ansätze wird unter Berücksichtigung der Kompatibilität ebenfalls praktiziert: Um zu besseren Ergebnissen in Randnähe zu gelangen, setzt man dort Dreieck-Elemente ein.

Das isoparametrische Elementkonzept schließlich versucht zusätzlich, bei der Gestaltgebung der Elemente der betrachteten Geometrie selbst Rechnung zu tragen. Dazu wird elementweise im Element-Inneren ein lokales Koordinatensystem dergestalt betrachtet, daß (im Falle eines ViereckElementes) das jeweilige Element durch das Einheitsquadrat parametrisiert wird. Die Klasse der dabei zugelassenen Koordinatentransformationen ist dabei an die Gestalt der Ansatzfunktionen gebunden: Im Falle bilinearer Elementfunktionen sind nur affine Koordinatentransformationen zulässig, wodurch die Linearität des Elementrandes erhalten bleibt. Für höhergradige Ansatzfunktionen wird dadurch jedoch erreicht, daß zusätzlich zur Verbesserung der Approximationsgenauigkeit der gesuchten Lösungsfunktion auch der Randverlauf des Elementes zwischen den Knoten krummlinig werden kann. Neben der isoparametrischen Transformation können auch noch weitere Geometrietransformationen betrachtet werden: Von subparametrischen Elementen spricht man, wenn der Polynomgrad dabei niedriger als jener der Approximation der Feldfunktion angesetzt wird. Analog dazu liegt beispielsweise bei linearer Ansatzfunktion und kubischer Geometrietransformation ein superparametrischer Ansatz vor. Die Vorteile hinsichtlich der geometrischen Approximationsgüte des (globalen) Gebietsrandes und damit auch die Verbesserungsmöglichkeiten bei Implementierung der Randbedingungen liegen auf der Hand, die Herleitung der Bedingungen an die Koeffizienten $c_{i}$ sowie die Koppelung der Elemente läßt sich mit vertretbarem Aufwand bewerkstelligen.

Die vorgestellte Methode der konformen Abbildung zeichnet sich dadurch aus, daß mittels globaler Koordinatentransformation operiert und die Berandung mit höherer Genauigkeit abgebildet wird.

\footnotetext{
${ }^{1}$ Die nachfolgenden Überlegungen verwenden die Ausführungen in

Hahn, M., ReCK, M., Kompaktkurs Finite Elemente für Einsteiger.

Springer, Wiesbaden, 2018. ISBN-13 978-3658227746
} 


\subsection{Einsatz auf verschiedenen weiteren Geometrien}

Es scheint abschließend noch angebracht, dem interessierten Leser Teile der im Laufe der Jahre durchgeführten Experimente zusammenzustellen. Auf Grund der damals weniger genauen Berechnungsmöglichkeiten der Ränderzuordnungsfunktion und teilweise noch unter Verwendung von $C$ Codierung sind die Ergebnisse zwar mit einem relativ großen numerischen Fehler behaftet, zeigen aber bereits die prinzipielle Durchführbarkeit der Methode auf. Für einen umfangreichen Überblick über konforme Abbildungen in geschlossener Form siehe [6], hier sucht man sich geeignete Teststellungen zur Überprüfung der implementierten Verfahren. Im übrigen hat sich der Versuch, den Einheitskreis selbst zur Parametrisierung der Geometrie und somit als Rechengitter heranzuziehen, wegen der Singularität im Ursprung als ein für unsere Zwecke weniger geeigneter Ansatz erwiesen.

Ellipse. Neben der gespiegelten Ellipse eignet sich als Ausgangsproblem auch die Ellipse selbst. Zwar läßt sich die Abbildung des Kreisinneren auf das Ellipseninnere über elliptische Funktionen angeben [6, S.47], die metrischen Größen für die Simulation gewinnt man aber auch, indem man beachtet, daß durch $\zeta=\sin z$ das Rechteck direkt auf die Ellipse abgebildet werden kann [6, S.46].

Kreiszwickel. Für das Innere des Kreisbogenzweiecks mit Innenwinkel $\pi \vartheta$ (mit der Differenz $\vartheta=\vartheta_{2}-\vartheta_{1}$ des vorderen und hinteren Tangentenwinkels) und Grundpunkten $\pm a$ auf der reellen $\zeta$-Achse lautet die konforme Abbildung auf die obere $w$-Ebene [22, S.203f.]

$$
w=\left(e^{-i \pi \vartheta_{1}} \frac{\zeta-a}{\zeta+a}\right)^{1 / \vartheta}
$$

woraus man sich unter Zuhilfenahme der Möbius-Transformation 2.5 ohne Mühe die Abbildung auf den Einheitskreis errechnet. Abbildung 6.1 zeigt die Ergebnisse der Simulation der Wärmeleitungsgleichung (1.1) in Polarkoordinaten im Inneren des Kreiszwickels mit Newton- bzw. DirichletRandbedingungen auf den Kreisbögen. In der Nähe der Grundpunkte $\pm 1+i$ liegen die Punkte des transformierten Rechengitters viel zu weit voneinander entfernt, woraus zwar eine unschöne Visualisierung im Vergleich zur PDE-Toolbox resultiert, die Ergebnisse sind aber durchaus brauchbar.
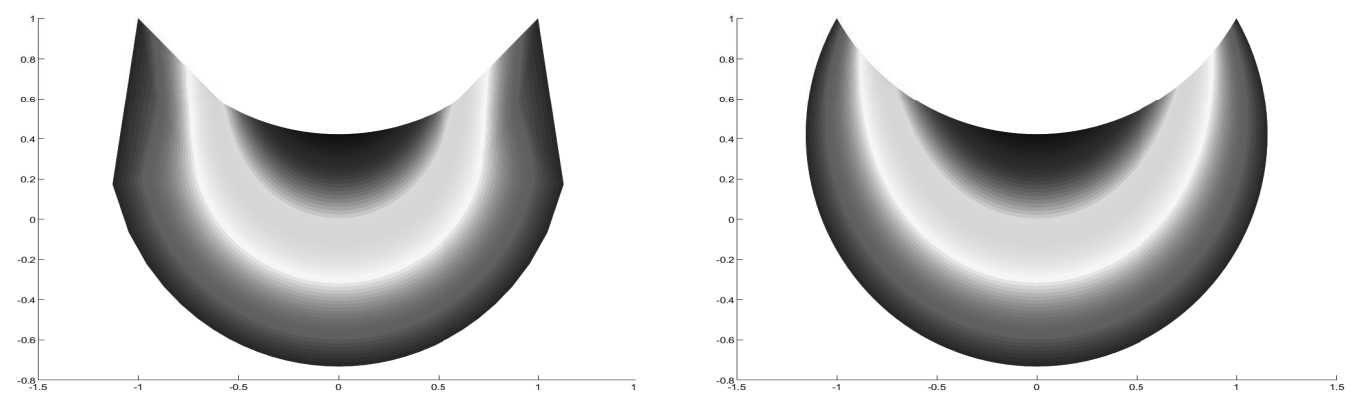

Abbildung 6.1: Wärmeleitung am Kreiszwickel: FD2 (links) im Vergleich mit PdeTbx (rechts).

Kreissektor. Die konforme Abbildung von der oberen $w$-Ebene auf das Innere des Kreissektors mit Öffnungswinkel $\pi \vartheta$ kann ebenfalls geschlossen angegeben werden [22, S.217ff.], sie lautet

$$
\zeta(w)=\left(\frac{1-\sqrt{w}}{1+\sqrt{w}}\right)^{\vartheta} .
$$

Die Ergebnisse für Ränderzuordnungsfunktion, Transformation von $\mathfrak{Q}_{121,121}$ und die resultierende Wärmeverteilung bei Vorgabe einer Dirichlet-Randbedingung $u_{0} \equiv 2$ zeigt Abbildung 6.2. Hier tritt der Fall ein, daß für eine ungerade Anzahl an Diskretisierungspunkten $(n=121)$ der Mittelpunkt der Unterseite des Rechenquadrates exakt auf den Punkt mit Spitze im Öffnungswinkel abgebildet wird. Dies stellt eine große Herausforderung für die numerische Konstruktionsmethodik dar, 
in der Tat führt der Weg über die iterative Lösung der Theodorsengleichung mittels sukzessiver Approximation zur Ermittlung der Ränderzuordnung bei Verwendung von trigonometrischen Interpolationspolynomen auf ein viel zu ungenaues Ergebnis. Der Fall wurde hinsichtlich einer Genauigkeitssteigerung nicht weiter untersucht, analog den Ausführungen in Abschnitt 3.3 zur Behandlung der numerischen Quadratabbildung könnte aber die Iteration durch sukzessive Approximation mit geeigneteren Basisfunktionen nach Analyse des Verhaltens in den drei singulären Punkten in Angriff genommen werden. Da in diesen Punkten zudem auch der Fundamentaltensor singuläres Verhalten aufweist, wird im Zuge der Simulation bei Randbedingungen, die über den Auswärtsnormalenvektor vorgeschrieben sind, dort ebenfalls eine gesonderte Untersuchung notwendig werden.
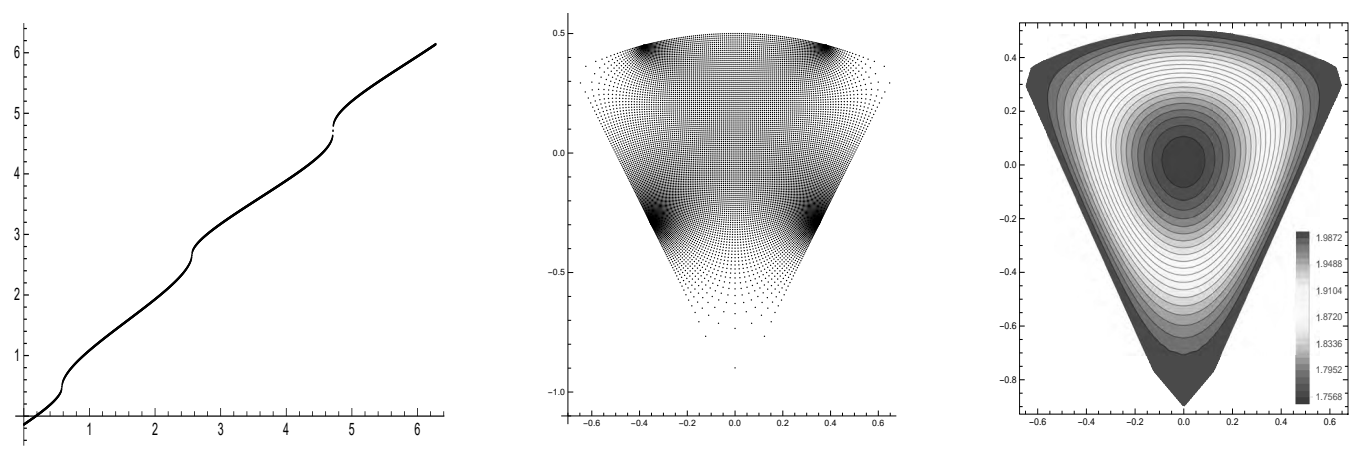

Abbildung 6.2: Untersuchungen am Kreissektor.

Polygonzug. Zur Abbildung des Kreises auf das unregelmäßige Sechseck aus Abbildung 6.3kann man im ersten Schritt die vorgegebenen Drehwinkel $\beta_{k}$ in Formel 2.4 einsetzen. Für die Lösung des Parameterproblems, somit zur Bestimmung der unimodularen Urbilder $w_{k}$ der Polygoneckpunkte, wurde dann die in der Fußnote Abschnitt 2.4 erwähnte Schwarz-Christoffel-Toolbox eingesetzt. Generell kann also im Falle von Polygonabbildungen resümiert werden, daß die numerische Konstruktion der Transformation über den Weg einer hochgenauen Lösung des Parameterproblems den aussichtsreicheren Zugang, verglichen mit der Iteration der Theodorsengleichung, darstellt. Weiters halten wir fest, daß bei unregelmäßigen Polygonzügen wegen des Parameterproblems keine geschlossene Darstellung, wie sie im Falle des Einheitsquadrates über (2.6) bzw. 2.7) hergeleitet wurde, mehr möglich ist. Sind die Werte $w_{k}$ jedoch hinreichend genau ermittelt und die Parameter für Translation und Drehstreckung fixiert, so kann Mathematica direkt mit Formel (2.4) operieren.
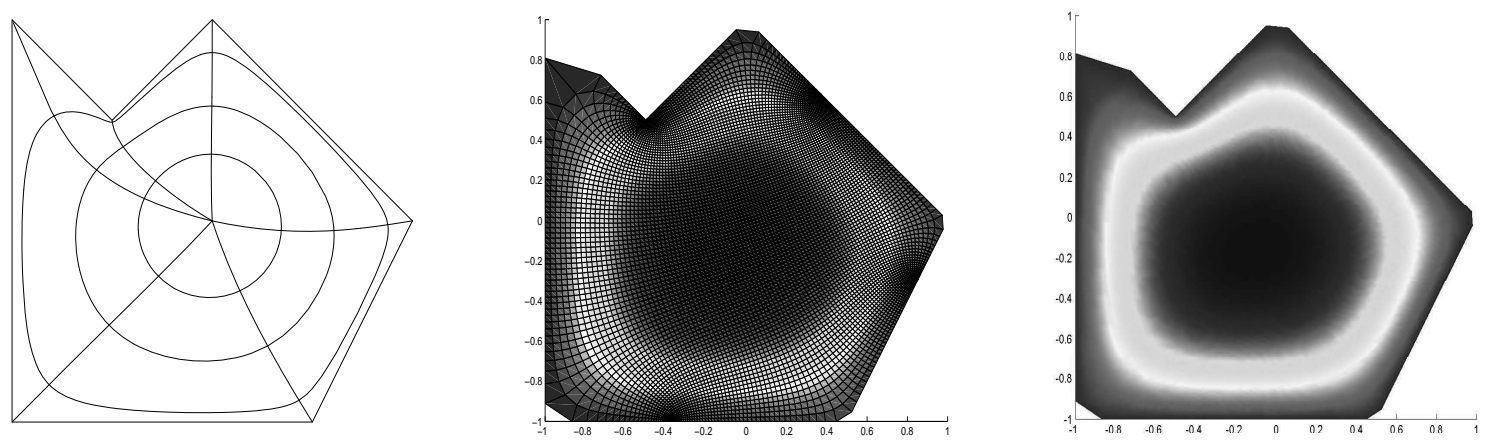

Abbildung 6.3: Transformation des Einheitskreises (links), Abbildung des Quadratgitters (Mitte) und Temperaturverteilung (rechts) für ein unregelmäßiges Sechseck. 
Geometrie ohne Rotationssymmetrie. Aus dem frühen Anfangsstadium der Untersuchungen stammt die in Abbildung 6.4 dargestellte Transformation. Von primärem Interesse war für uns, ob und wie weit sich die gespiegelte Ellipse $(p=0.6)$ auf die dargestellte Geometrie mit $\varepsilon>1$ sukzessive deformieren lässt. Als Startwerte für das Newtonsche Verfahren wurden dabei die iterierten Ränderzuordnungsfunktionen des jeweils vorhergehenden Deformationsschrittes verwendet.

Die Fragestellung entstand im Zuge einer Kooperation mit dem Österreichischen Forschungszentrum in Seibersdorf. Dort war man primär mit der praktischen Untersuchung und Nachbildung von schadstoffarmen Verbrennungsprozessen in rotierenden Wirbelschicht- und Drehrohröfen befaßt, die theoretischen Untersuchungen dazu stießen aber dennoch auf einiges Interesse. Daher wurde mit den damals zur Verfügung stehenden Datenstrukturen der Programmiersprache $C$ die konforme Abbildung des Einheitskreises auf den Querschnitt eines Wirbelschichtofens realisiert.

Der Versuch einer Simulation von mehrphasigen Flüssen auf dem Querschnitt des unten abgebildeten Gebietes, modelliert mit Hilfe der NAVIER-StOKEsschen Gleichungen, hat sich damals allerdings als etwas zu ambitioniert herausgestellt: Einerseits waren der hochgenauen Berechnung der metrischen Größen in den letzten Jahren des vorigen Jahrtausends noch ihre engeren Grenzen gesetzt und rückblickend kann zudem festgestellt werden, daß eine Parametrisierung der Geometrie mit Hilfe des Einheitskreises keinen optimalen Zugang zum Problem darstellt.
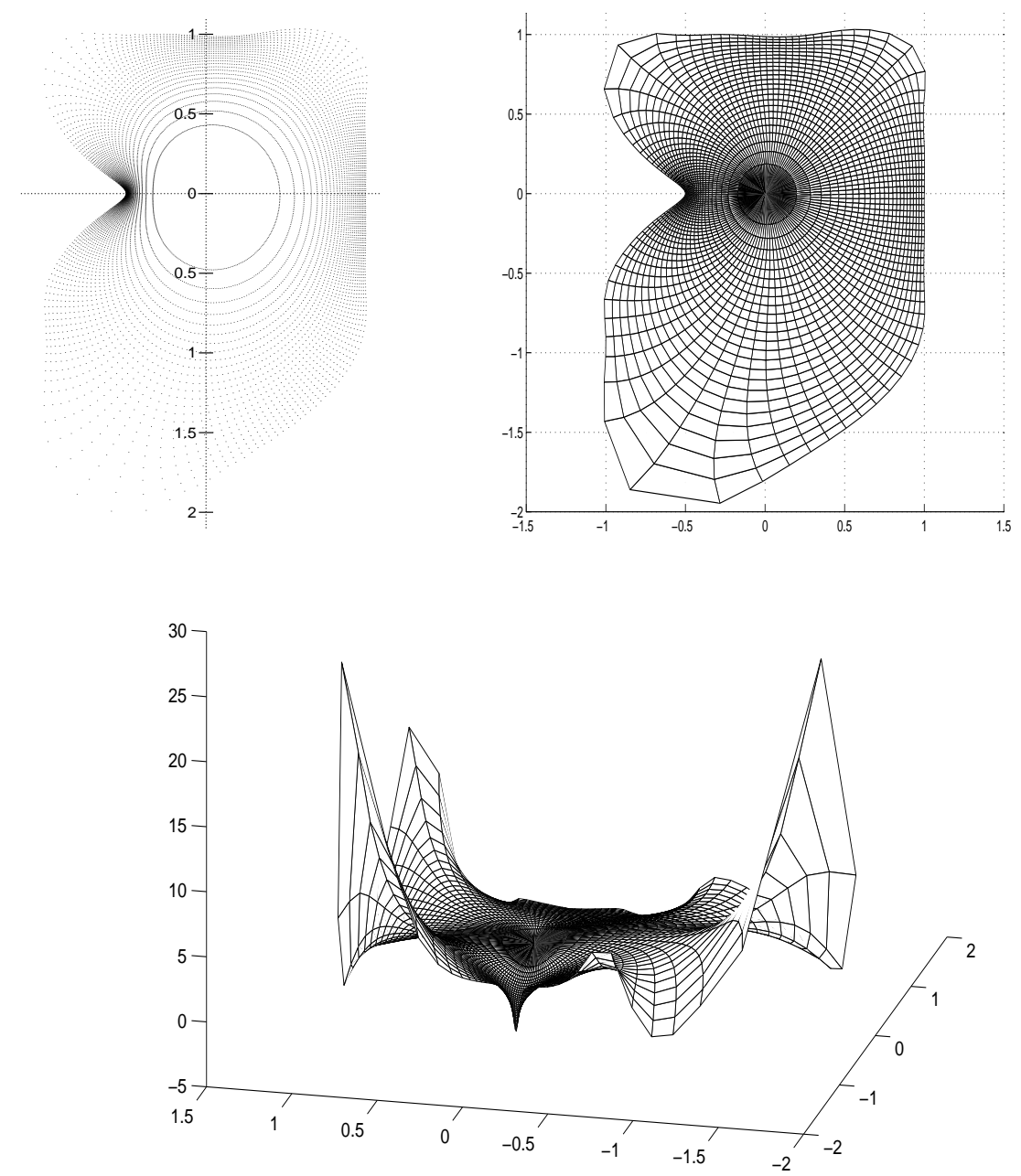

Abbildung 6.4: Konforme Abbildung des Einheitskreises und Zusammenhangskoeffizient $\Gamma_{11}^{1}$ einer Geometrie, die dem Querschnitt einer rotierenden Wirbelschicht-Verbrennungsanlage entspricht. 


\subsection{Genauigkeitssteigerung der Ränderzuordnungsfunktion}

Mit der Fragestellung, wie sich die in Unterabschnitt 3.1.1 angeführten zwei Varianten zur Erhöhung der Approximationsgüte von $\theta(\varphi)$ zur Lösung der Theodorsengleichung (2.14) weiterentwickeln lassen, ist ein interessanter Forschungsschwerpunkt im Feld der hochgenauen numerischen Behandlung nichtlinearer FREDHOLMscher Integralgleichungen zweiter Art gegeben, die allgemein in der Form

$$
\theta(\varphi)=f(\varphi)+\int_{a}^{b} \mathfrak{K}(\theta(y), \varphi, y) \mathrm{d} y
$$

mit festen Integrationsgrenzen, nichtlinearem Kern $\mathfrak{K}$ und gesuchter Funktion $\theta(\varphi)$ vorliegen. Ein vielversprechender Ansatz scheint die Verwendung einer dynamisch-adaptiven Diskretisierungsmethode zu sein, in Abhängigkeit gewisser Kriterien (etwa der Schwankung einer beteiligten Hilfsfunktion um einen Gitterpunkt) werden dabei dem äquidistanten Basisgitter im Zuge des Iterationsprozesses Punkte zu- oder weggeschaltet. In der ersten Variante führt dies in den Bereich der trigonometrischen Interpolation auf nicht-äquidistanten Gittern, für den zweiten Fall der direkten Berechnung des Hauptwertintegrals an den Stellen und anschließender Interpolation wurden dazu ansatzweise einige Versuche ausprogrammiert Abbildung 6.5).

In dieser Arbeit wurde ausschließlich Gleichung 2.14 zur Gewinnung der Ränderzuordnung verwendet. Wie sich gezeigt hat, sind einer solchen Vorgehensweise in Abhängigkeit der in Betracht stehenden Geometrie (Sternförmigkeit, $\varepsilon$-Bedingung) jedoch Grenzen gesetzt. Sollte sich durch diese Restriktionen nicht die gewünschte Genauigkeit im Iterationsprozeß einstellen, so findet man in der Literatur eine Vielzahl an Alternativen, um zu einer konformen Abbildung des Quadrates auf die Geometrie zu gelangen. Aus dem Werk von GAIER [15, Kap.I, §2] im Zuge von Voruntersuchungen testweise implementiert wurden die Integralgleichung von WARSCHAWSKI und STIEFEL [(2.22),ebd.], die Gerschgorinsche Gleichung [(2.9),ebd.] sowie die StieltJes-Gleichung [(2.25),ebd.] als Vorstufe zur ARBENZschen Integralgleichung. Alle diese Gleichungen sind im Gegensatz zur Integralgleichung von TheOdorsen linearer Natur in dem Sinne, daß ihr Kern nur linear von der gesuchten Funktion abhängt. Es liegt dies darin begründet, daß sich die Konstruktionsmethoden den umgekehrten Fall der konformen Abbildung eines Gebietes auf den Einheitskreis zur Aufgabe stellen.

Immer aber wird die Güte der Simulation selbst in essentiellem Zusammenhang mit der Lösungsqualität der zur Ermittlung der Ränderzuordnungsfunktion involvierten Integralgleichungen stehen.

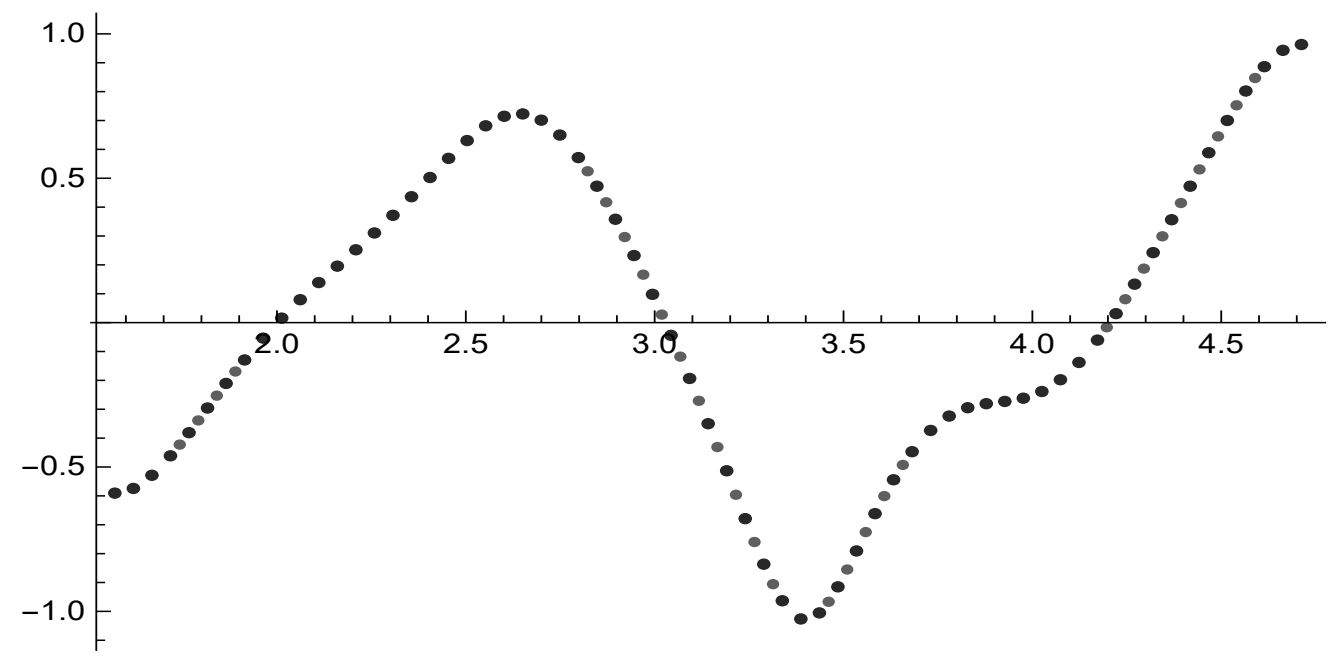

Abbildung 6.5: In Abhängigkeit der Variation von $\mathcal{K}[\beta](\varphi)$ können im Zuge kontinuierlich durchgeführter Iterationen Zusatzpunkte (rot) zur Erhöhung der Genauigkeit einprogrammiert werden. 


\section{Literaturverzeichnis}

[1] Ablowitz, M.J., Fokas, A.S., Complex Variables: Introduction and Applications. Cambridge University Press, Cambridge, 1997, ISBN 0-521-48058-2 (hb).

[2] Betz, A., et al., Konforme Abbildung. Springer-Verlag, Berlin/Göttingen/Heidelberg, 1964 (2. Auflage), LCCCN 63-22377.

[3] Blümlinger, M., Konforme Abbildungen von Gebieten mit Ecken. TU Wien, Dipl.-Arb., 1984, http://katalog.ub.tuwien.ac.at/AC00227190

[4] Breitenecker, F., Ecker, H., Bausch-Gall, I., Simulieren mit ACSL. Vieweg, Braunschweig/Wiesbaden, 1993, ISBN 3-528-06381-5.

[5] Brown, J.W., Churchill, R.V., Complex Variables and Applications, Eight Edition. McGraw-Hill, New York, 2009, ISBN 978-0-07-305194-9.

[6] Dalichau, H., Konforme Abbildung und elliptische Funktionen. https://www.dalichau.com/app/download/2627383/Konf_Abb_Vs_1_3.pdf München, 1993.

[7] Dirschmid, H.J., Mathematische Grundlagen der Elektrotechnik. Vieweg, Braunschweig/Wiesbaden, 1988, ISBN 3-528-33034-1.

[8] Dirschmid, H.J., Tensoren und Felder. Springer-Verlag, Wien, 1996, ISBN 3-211-82754-4.

[9] Douschan, P., Benefit der Anwendung des Diodenlasers in der Endodontie. Med. Univ. Graz, Diplomarbeit, 2014.

[10] Driscoll, T.A., Schwarz-Christoffel Toolbox User's Guide. http://www.math.udel.edu/ driscoll/SC/guide.pdf

[11] Ferziger, J.H., Perić, M., Computational Methods for Fluid Dynamics. Springer-Verlag, Berlin-Heidelberg, 1996, ISBN 3-540-59434-5.

[12] Fornberg, B., Generation of Finite Difference Formulas on Arbitrarily Spaced Grids. Mathematics of Computation, Vol. 51, Number 184, pp. 699-706, 1988. American Mathematical Society 0025-5718/88.

[13] Fornberg, B., Calculation of Weights in Finite Difference Formulas. SIAM Rev., Vol. 40, No. 3, pp. 685-691, 1998, ISSN (print): 0036-1445.

[14] Fornberg, B., Ghrist, M., Spatial Finite Difference Approximations for Wave-Type Equations. SIAM J. Numer. Anal., Vol. 37, No. 1, pp. 105-130, 1999, ISSN (print): 0036-1429.

[15] GaIER, D., Konstruktive Methoden der konformen Abbildung. Springer-Verlag, Berlin-Göttingen-Heidelberg, 1964. 
[16] Hassenpflug, W.C., Elliptic Integrals and the Schwarz-Christoffel Transformation. Computers \& Mathematics with Applications, Vol. 33. 15-114. 10.1016/S0898-1221(97)00091-6.

[17] Henrici, P., Applied and Computational Complex Analysis, Volume I. John Wiley \& Sons, New York-London-Sydney-Toronto 1974, ISBN 0-471-37244-7.

[18] Herod, J.V., Asynchronous Studies in Undergraduate Partial Differential Equations. Lecture 33: The Heat Equation on a Disk. https://people.math.gatech.edu/ herod/conted/M33.pdf

[19] Holzinger, M., Modellorientierte Parallelisierung von Modellen mit verteilten Parametern. Diplomarbeit, TU Wien, 1996, 554802 II.

[20] Howell, L.H., Trefethen, L.N., Kleitman, D.J., Helgason, S., Computation of Conformal Maps by Modified Schwarz-Christoffel Transformations. Massachusetts Institute of Technology 1990 (PHD Thesis).

[21] HÜBNER, O., The Newton method for solving the Theodorsen integral equation. Journal of Computational and Applied Mathematics 14 (1986), pp. 19-30.

[22] Koppenfels, W.v., Stallmann, F., Praxis der konformen Abbildung. Springer-Verlag, Berlin-Göttingen-Heidelberg 1959.

[23] Kythe, P.K., Computational Conformal Mapping. Birkhäuser, Boston 1998, ISBN 0-8176-3996-9.

[24] Martins, M.A., Oliveira, L.S., Valle, R.M., Adaptivity for finite volume on unstructured triangular meshes: a study on thermal injury in teeth. International Journal for Numerical Methods in Engineering, 2004; 61(10): 1625-1643.

[25] Nehari, Z., Conformal Mapping. McGraw-Hill, New York, 1952, First Edition, LCCCN 51-12633.

[26] Panas, A.J., Żmuda, S., Terpilowski, J., Preiskorn, M., Investigation of the Thermal Diffusivity of Human Tooth Hard Tissue. Intl. Journal of Thermophysics, Vol. 24, Number 3, pp. 837-848, 2003. Online ISSN 1572-9567.

[27] Priwalow, I.I., Randeigenschaften Analytischer Funktionen. Hochschulbücher für Mathematik, Band 25 (2. Auflage). Deutscher Verlag der Wissenschaften, Berlin, 1956.

[28] Richardson, S., Integral Equations. Mathematica Journal, Vol. 9, Issue 2, pp. 460-482, 2004. https://www.mathematica-journal.com/issue/v9i2/contents/IntegralEquations/IntegralEquations.pdf

[29] Schiesser, W.E., The Numerical Method Of Lines. Integration of PDEs. Academic Press Inc., San Diego, 1991, ISBN 0-12-624130-9.

[30] Spiegel, M.R., Komplexe Variablen. Theorie und Anwendung. Mit einer Einführung in die Konformen Abbildungen und ihre Anwendungen.

McGraw-Hill, Hamburg, 1982, ISBN 0-070-92016-8.

[31] Trefethen, L., Numerical Computation of the Schwarz-Christoffel Transformation. SIAM Journal on Scientific and Statistical Computing, 1(1), 82-102. (1980).

[32] Trefethen, L.N., Driscoll, T.A., Schwarz-Christoffel mapping in the Computer Era. Proceedings of the International Congress of Mathematicians, Vol. III (Berlin, 1998), vol. 1998, pp. 533-542, ISSN 1431-0643.

[33] Warschawski, S.E., On a Theorem of L. Lichtenstein. Pacific Journal of Mathematics, Vol. 5, 835-839, 1955.

[34] Wegmann, R., Discretized versions of Newton type iterative methods for conformal mapping. Journal of Computational and Applied Mathematics 14 (1986), pp. 207-224. 


\title{
Curriculum Vitae
}

\author{
MARTIN HOLZINGER
}

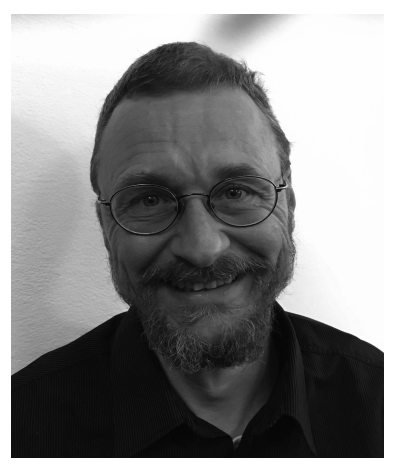

\section{Angaben Zur Person}

Familienstand: ledig, eine Tochter

Staatsbürgerschaft: Österreich

Geburtsdatum/-ort: 18. August 1970 / Vöcklabruck, OÖ

\section{Schulische Ausbildung}
$1976-1980$
Volksschule Regau
$1980-1988$
Unter-/Oberstufe Bundesgymnasium Vöcklabruck (neusprachliche Richtung)
6. 6.1988
Ablegung der Reifeprüfung mit ausgezeichnetem Erfolg

\section{Berufliche und AKademische LaufBahn}

1988 - 1996 Studium der Technischen Mathematik an der TU Wien

18. 4. 1996 Sponsion zum Diplom-Ingenieur der Technischen Mathematik an der TU Wien

Thema: $\quad$ Modellorientierte Parallelisierung von Modellen mit verteilten Parametern

Betreuung: Ao.Univ.-Prof. Dr. FELIX BREITENECKER

ab 1996 Doktoratsstudium der Technischen Wissenschaften an der TU Wien (Institut E101)

Teilnahme an diversen internationalen Tagungen

1996-2000 Zusammenarbeit mit dem Österreichischen Forschungszentrum in Seibersdorf 1999-2005 Studienassistent am Institut E101 (Analysis und Technische Mathematik), TU Wien KPMG-Consulting, Studie zum Thema E-Government

2000-2010 ZID TU Wien/Abt. Standardsoftware: Software-Setup, Schwerpunkt Microsoft

2009-2011 Lektor an der FH Technikum Wien: Vorlesungen über Simulationstechnik

2010-2016 ZID TU Wien/Abt. Standardsoftware: Referatsleiter Campussoftware (8 MA)

2017-2018 ZID TU Wien: Leiter der Abteilung Standardsoftware (20 MA)

2019

Bildungskarenz zur Fertigstellung der Dissertation

\section{PRÄSENZDIENST}

28.3.-30.11.1998 Stiftskaserne, Landesverteidigungsakademie/Heeres-Datenverarbeitungsamt

\section{ZusÄtZliche QuALifikATIONEN}

Englisch: $\quad$ fließend in Wort und Schrift

IT: $\quad$ Administrator Windows/Linux Server, MS Office, Virtualisierungsumgebungen FORTRAN, C/C++, PowerShell, LaTex, Matlab, Mathematica, Atmel (Franklin) 




\section{Über den Autor}

Martin Holzinger studierte Technische Mathematik an der TU Wien, seine Diplomarbeit hatte 1996 die Parallelisierbarkeit der Linienmethode zum Thema. Neben seiner Verbundenheit mit dem Institut für Analysis und Scientific Computing, Vorlesungen an Fachhochschulen und der Zusammenarbeit mit dem ARC Seibersdorf arbeitet er seit dem Jahr 2000 haupt-

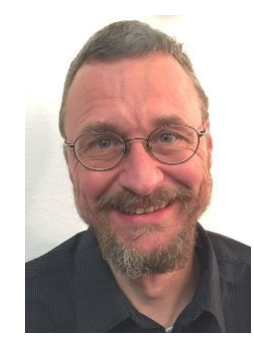
beruflich am Zentralen Informatikdienst der TU Wien. Die Hobbies des 1970 geborenen Oberösterreichers sind Schach, Bergwandern, Fliesenlegen und die konforme Abbildung.

\section{Über dieses Buch}

Ein charmanter Zugang zur Simulation partieller Differentialgleichungen abseits der etablierten Methode der Finiten Elemente eröffnet sich durch Synthese von konformer Abbildung, CTDS-Linienmethode und einer hochentwickelten Programmierumgebung (Mathematica). Daß der Weg mit der CTDS-Methode prinzipiell zielführend ist, wird zunächst an Hand der Wärmeleitungsgleichung am Einheitsquadrat demonstriert: Die Ergebnisse des präsentierten Simulationscodes werden mit einer berechneten Reihenlösung verglichen, die Approximation der Ortsableitungen erfährt hinsichtlich Konvergenzordnung en passant noch eine wesentliche Erweiterung. Die konforme Abbildung, zur Konstruktion aufgefasst als Punkttransformation und mit Hilfe der Zwischenschaltung des Einheitskreises durch Lösen der Theodorsengleichung anwendbar auf sternförmige Gebiete, leistet als Koordinatentransformation die Parametrisierung zweidimensionaler Gebiete durch das Einheitsquadrat. Die Konstruktionsmöglichkeiten solcher Abbildungen werden in Theorie und Praxis eingehend erörtert. Reisen Sie dann mit in die Welt der Riemann'schen Geometrie mit durchaus überraschenden Ergebnissen. Das abschließende Kapitel sollte Pflichtlektüre für unregelmäßige Zähneputzer sein!

\section{About the Series}

The ASIM series Advances in Simulation / Fortschrittsberichte Simulation presents new and recent approaches, methods, and applications in modelling and simulation. The topics may range from theory and foundations via simulation techniques and simulation concepts to applications. As the spectrum of simulation techniques and applications is increasing, books in these series present classical techniques and applications in engineering, natural sciences, biology, physiology, production and logistics, and business administration, upcoming simulation applications in social sciences, media, data management, networking, and complex systems, and upcoming new simulation techniques as agent-based simulation, co-simulation, and deep learning, etc.

The series puts emphasis on monographs with special character, as $\mathrm{PhD}$ theses, habilitation treatises, project reports and overviews on scientific projects. ASIM - Arbeitsgemeinschaft Simulation, the German Simulation Society (part of GI - Gesellschaft für Informatik) has founded the series Advances in Simulation / Fortschrittsberichte Simulation together with ARGESIM Publisher Vienna in order to provide to the international simulation community a quick and cost-efficient print and e-book series with open access.

ISBN print

ISBN 978-3-903311-10-7

TU Verlag, Vienna, 2020 www.tuverlag.at
ISBN ebook

DOI ID

ISBN 978-3-903347-36-6 DOI 10.11128/fbs.36

ARGESIM Publisher, Vienna, 2020

www.argesim.org 